\title{
Amchitka Island Environmental Analysis - Phase 4 Actinide Analysis at Idaho National Laboratory
}

G. Elias

B.K. Schuetz

J.G. Eisenmenger

A.L. Freeman

M.E. Mcllwain

November 2005

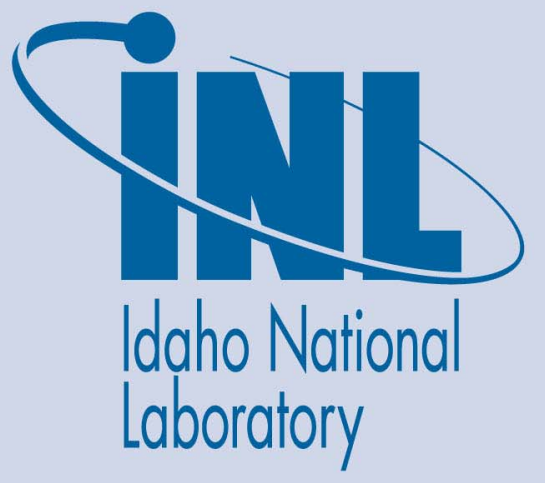

The INL is a U.S. Department of Energy National Laboratory operated by Battelle Energy Alliance 
INL/EXT-05-00935

\title{
Amchitka Island Environmental Analysis - Phase 4 Actinide Analysis at Idaho National Laboratory
}

\author{
G. Elias \\ B.K. Schuetz \\ J.G. Eisenmenger \\ A.L. Freeman \\ M.E. Mcllwain
}

November 2005

\begin{abstract}
Idaho National Laboratory
Idaho Falls, Idaho 83415
\end{abstract}

Prepared for the

U.S. Department of Energy

Assistant Secretary for Environmental Management

Under DOE Idaho Operations Office

Contract DE-AC07-05ID14517 


\title{
Amchitka Island Environmental Analysis- Phase 4 Actinide Analysis at Idaho National Laboratory
}

\begin{abstract}
Summary
Idaho National Laboratory (INL) provided support to Vanderbilt University and Consortium for Risk Evaluation with Stakeholder Participation (CRESP) in their activities, supported by the Department of Energy (DOE), to assess the impact of past nuclear testing at Amchitka Island on the ecosystem of the island and surrounding ocean. INL participated in this project in four phases. INL support in the first three phases is documented in Report \# INL/EXT-05-00361. The details of INL participation in Phase 4 (sample results, Quality Control (QC) results, and other related quality assurance documents) are included in this report.
\end{abstract}





\section{CONTENTS}

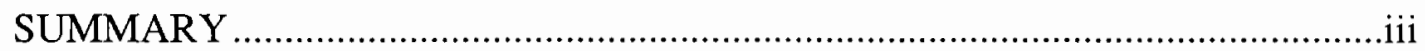

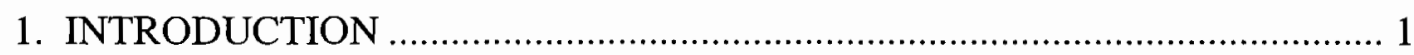

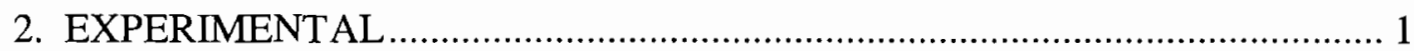

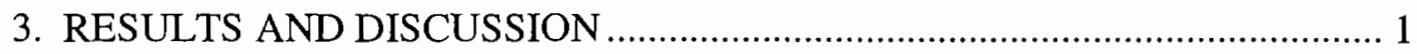

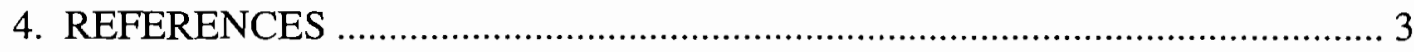




\section{Amchitka Island Environmental Analysis - Phase 4 Actinide Analysis at Idaho National Laboratory}

\section{INTRODUCTION}

The Idaho National Laboratory (INL) operated by Battelle Energy Alliance (BEA) was tasked by Consortium for Risk Evaluation with Stakeholder Participation (CRESP) via CRESP/Department of Energy Idaho Operations Office (DOE-ID) interagency agreements to perform several radioisotope measurements to assess the impact of past nuclear testing at Amchitka Island on the ecosystem of the island and surrounding ocean. INL supported CRESP in four phases. INL support in phases 1 through 3 are documented in Report \# INL/EXT-05-00361.

At INL, the Science and Technology organization has the gamma, alpha, and Inductively Coupled Plasma Mass Spectrometry (ICP-MS) capabilities available to measure the activity of these radioisotopes. The target list for INL in phase 4 consisted of Am-241, $\mathrm{Pu}-238, \mathrm{Pu}-239 / 240, \mathrm{U}-234, \mathrm{U}-235, \mathrm{U}-236$, and U-238 nuclides. The data collected for these radioisotopes in different biological matrices (soft tissue and kelp) from Amchitka and the internal and external QC information are documented in this report.

\section{EXPERIMENTAL}

The procedures and documents used for the project are in the Method Manual ${ }^{1}$. In radiochemistry, the analytes in samples were separated by complete ashing and dissolution of the samples using procedure ACMM- $3816^{2}$ in the Method Manual.

\section{RESULTS AND DISCUSSION}

Details of the phase 4 alpha analyses, which include data package for the batches and quality assurance/quality control summary (Internal and External QA/QC documentation), are found in this report. All QA/QC checks were performed in accordance with the QA plan ${ }^{3-4}$.

\section{Radiochemistry Alpha analysis}

In phase 4, there were four batches (batch 13-16) of samples for radiochemical analysis. The samples were analyzed for Am-241, Pu-238, Pu-239/240, U-234, U-235, U-236 and U-238. Complete ashing and dissolution of the samples were achieved and no unusual steps were taken in performing the alpha analyses. The results in this report are the same as those reported as preliminary results to CRESP during October 2005 - November 2005 . 
The actinide procedure for small solid sample sizes in ACMM- $3816^{2}$ was used for alpha analysis. Batch 13 and 16 were soft tissue and batch 14 and 15 were kelp samples. Batch 13 (soft tissue) and batches 14 and 15 (kelp) discolored the platinum dishes during fusion. The reason for this phenomenon is unknown. Since this was observed only in the marine samples, there is reason to think that the high chloride content with the other acids added for digesting the samples can had an effect. Several other batches of kelp and soft tissues did not cause this problem. Therefore, this can be specific to certain species or certain locations where these samples were collected. In batch 14, low tracer yield for all analytes was observed in K-AA-618 due to sample loss during the process, and relatively lower yield of Pu was observed in K-AA-611, K-AA-616, K-BB-600, K-BB-602, and K$\mathrm{BB}-609$, possibly due to the loss of some Pu fraction to Thorium fraction.

Internal and external quality control (QC) results, and the internal QC checks for the detectors used to count these samples are also included in this report. Results show that all relevant parameters were "in control" during the sample counting time frame. Results from the external QC intercomparison program are also documented here. 


\section{REFERENCES}

(1) INEEL Method Manual for the Amchitka Environmental Sample Analysis, Revision 1, December 2004.

(2) ACMM 3816 - Determination of Selected Actinide Nuclides and Strontium-90 in Filters and Solids.

(3) PLN-1719, "Quality Assurance Project Plan for the Analysis of Amchitka Island Samples," Revision 1, January 2005.

(4) PLN-153, "Quality Assurance Project Plan for Analytical Laboratories Department Radioanalytical Activities," Revision 2, May 2004. 



\section{RADIOANALYTICAL ANALYSES DATA PACKAGE}

Project Title: $\quad$ Amchitka Island Alpha Analysis Report (Batch 13)

Lab Name:

RTC

Case No:

NA

Report No.:

AmchitBatchF13

Method Type: A/B

Approved SAP No:: NA

SDG No: $\quad$ S-R-283

\section{SAMPLE NUMBERS}

Customer Sample ID

\begin{tabular}{l}
\hline S-R-283 \\
\hline S-R-284 \\
\hline S-R-285 \\
\hline S-R-286 \\
\hline S-R-287 \\
\hline S-R-288 \\
\hline S-R-289 \\
\hline S-R-290 \\
\hline S-R-291 \\
\hline S-R-292 \\
\hline S-R-293 \\
\hline S-R-294 \\
\hline$S-R-295$ \\
\hline$S-R-296$ \\
\hline$S-R-297$ \\
\hline$S-R-298$ \\
\hline$S-R-299$ \\
\hline$S-R-300$ \\
\hline$S-R-301$ \\
\hline$S-R-302$ \\
\hline
\end{tabular}

Lab Sample

ID

02WH-01-A

02WH-02-A

02WH-03-A

02WH-04-A

02WH-05-A

02WH-06-A

02WH-07-A

02WH-08-A

02WH-09-A

02WH-10-A

02WH-11-A

02WH-12-A

02WH-13-A

02WH-14-A

02WH-15-A

02WH-16-A

02WH-17-A

02WH-18-A

02WH-19-A

02WH-20-A 

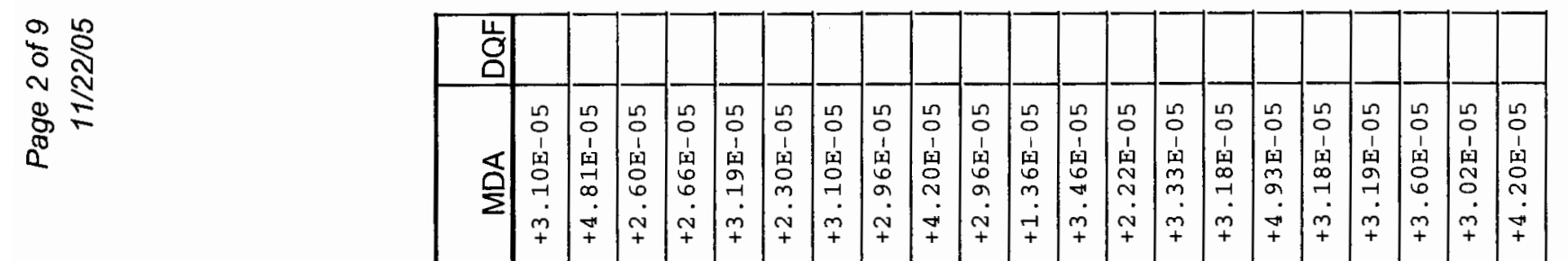
造

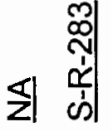

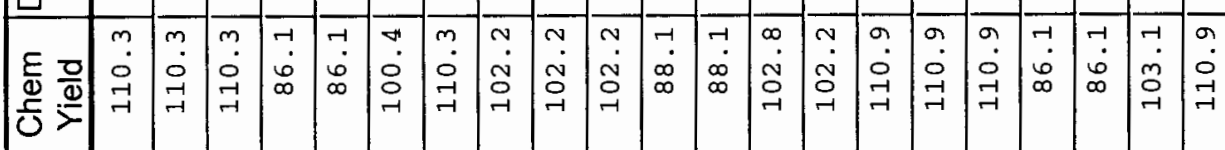
थ 范 $\frac{\mathrm{N}}{\infty}$

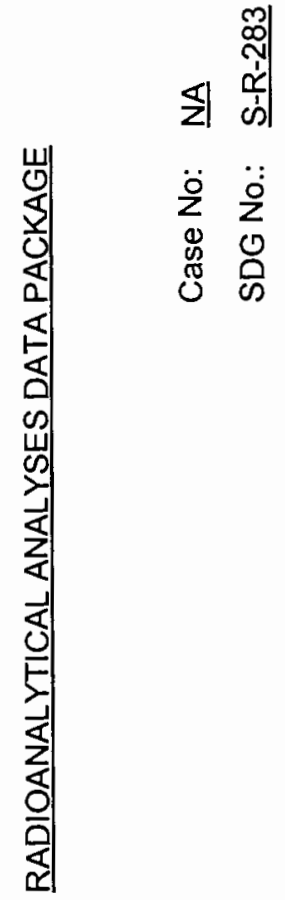

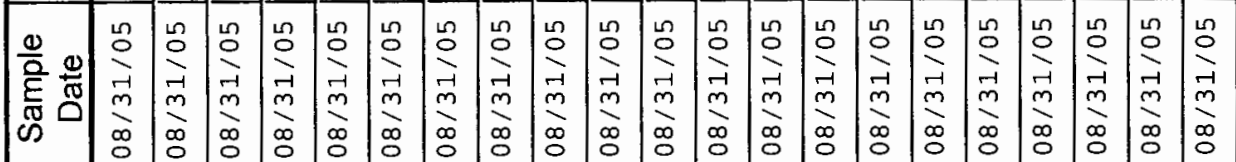

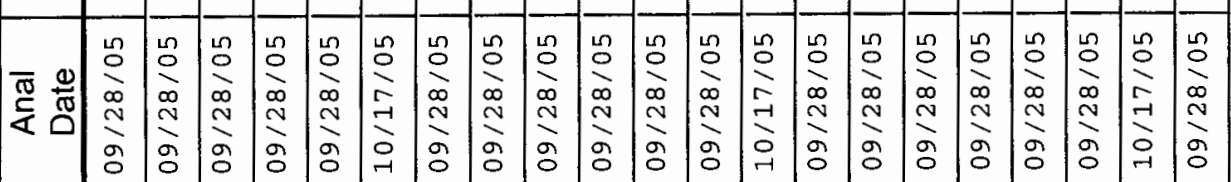
क

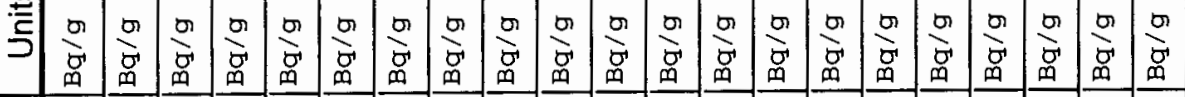

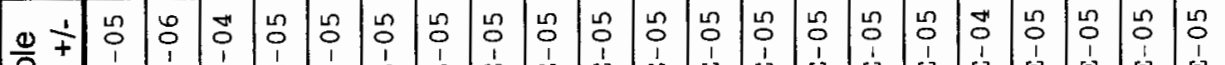

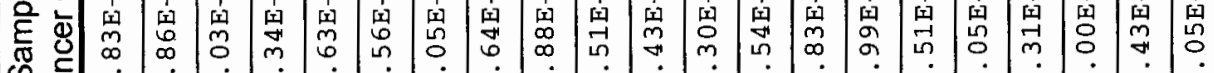

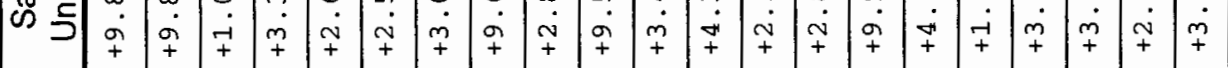
응

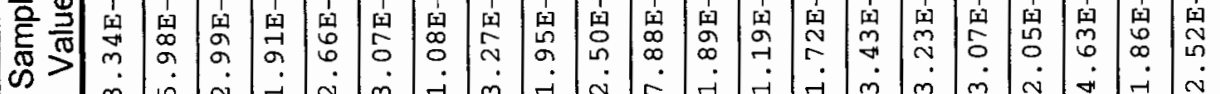

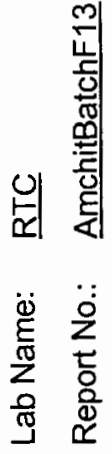

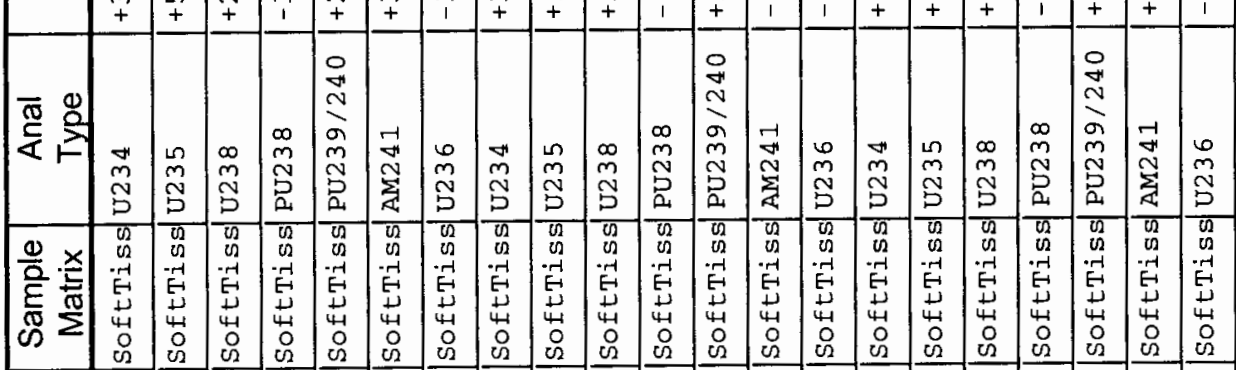

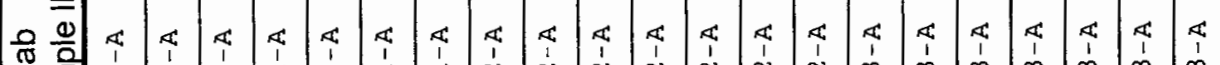

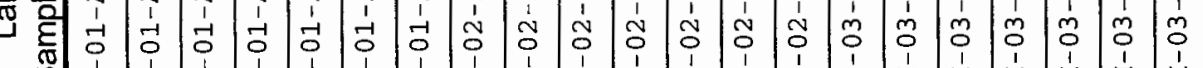

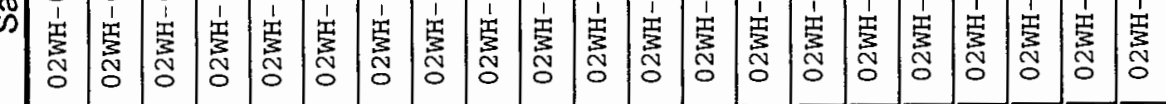




\begin{tabular}{|c|c|c|c|c|c|c|c|c|c|c|c|c|c|c|c|c|c|c|c|c|c|}
\hline $\begin{array}{l}\text { U. } \\
\\
\end{array}$ & & & & & & & & & & & & & & & & & & & & & \\
\hline$\frac{\mathrm{o}}{2}$ & $\begin{array}{l}\text { In } \\
0 \\
1 \\
1 \\
1 \\
5 \\
\vdots \\
+ \\
+ \\
+\end{array}$ & $\begin{array}{l}\Omega \\
0 \\
1 \\
1 \\
0 \\
0 \\
+ \\
-1 \\
+ \\
+\end{array}$ & $\begin{array}{l}n \\
0 \\
1 \\
\text { a } \\
5 \\
0 \\
+ \\
7 \\
+\end{array}$ & 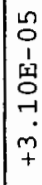 & $\begin{array}{l}2 \\
0 \\
1 \\
1 \\
\text { 19 } \\
0 \\
0 \\
0 \\
+ \\
+\end{array}$ & 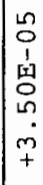 & 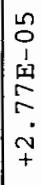 & 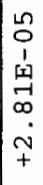 & 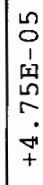 & 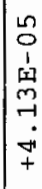 & $\begin{array}{c}n \\
0 \\
1 \\
w \\
\neq \\
+ \\
+ \\
\sim \\
+ \\
+\end{array}$ & $\begin{array}{l}n \\
0 \\
1 \\
1 \\
0 \\
0 \\
0 \\
\dot{p} \\
+\end{array}$ & 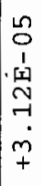 & 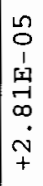 & 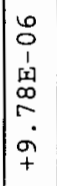 & $\begin{array}{l}\stackrel{n}{0} \\
1 \\
1 \\
\mathfrak{y} \\
\tilde{n} \\
m \\
\sim \\
+ \\
+\end{array}$ & $\begin{array}{l}0 \\
0 \\
1 \\
1 \\
0 \\
0 \\
\vdots \\
0 \\
9 \\
+\end{array}$ & $\begin{array}{l}n \\
0 \\
1 \\
1 \\
\text { I } \\
⿱ ㇒ \\
m \\
-1 \\
+\end{array}$ & 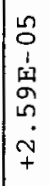 & 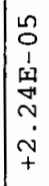 & 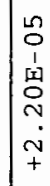 \\
\hline 高 & $\stackrel{n}{\circ}$ & $\stackrel{\llcorner}{\circ}$ & 告 & 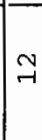 & 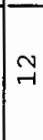 & $\underset{F}{F}$ & 농 & 10 & $10^{\circ}$ & 10 & $\stackrel{m}{\rightarrow}$ & $\stackrel{m}{\rightarrow}$ & $\rightarrow$ & 10 & 10 & 10 & o & |ت゙ & $\vec{r}$ & $\stackrel{\vec{H}}{-}$ & 15 \\
\hline $\begin{array}{l}\frac{E}{\Phi} \\
\frac{D}{U}\end{array}$ & $\begin{array}{l}0 \\
\dot{0} \\
\dot{0} \\
+\end{array}$ & $\begin{array}{l}0 \\
\dot{0} \\
\dot{0} \\
-1\end{array}$ & $\begin{array}{l}0 \\
\dot{0} \\
0\end{array}$ & $\begin{array}{l}\dot{\alpha} \\
\infty\end{array}$ & 今. & $\begin{array}{l}\overrightarrow{6} \\
\tilde{\sim} \\
ت \\
-1\end{array}$ & $\begin{array}{l}0 \\
\dot{0} \\
0 \\
0\end{array}$ & $\begin{array}{l}0 \\
0 \\
0 \\
0 \\
-1\end{array}$ & י & \begin{tabular}{l}
$n$ \\
. \\
\multicolumn{1}{c}{} \\
-1
\end{tabular} & $\begin{array}{l}\vec{\nabla} \\
\dot{\tilde{N}}\end{array}$ & $\begin{array}{l}\vec{N} \\
\dot{N}\end{array}$ & 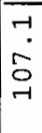 & 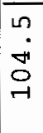 & 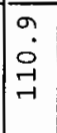 & 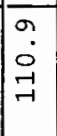 & $\begin{array}{l}\sigma \\
\dot{0} \\
-1 \\
-1\end{array}$ & $\begin{array}{l}\overrightarrow{1} \\
\dot{0} \\
\infty\end{array}$ & $\begin{array}{l}+4 \\
\vdots \\
\infty \\
\infty\end{array}$ & $\begin{array}{l}\overrightarrow{0} \\
\stackrel{\circ}{\circ} \\
-1\end{array}$ & $\begin{array}{l}\sigma \\
\ddot{\sigma} \\
\ddots \\
\ddots\end{array}$ \\
\hline 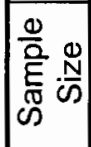 & $\stackrel{\stackrel{n}{\sim}}{\sim}$ & $\stackrel{10}{\sim}$ & 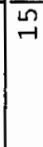 & $\underset{\sim}{n}$ & $\sqrt{n}$ & 号 & $\stackrel{\widetilde{m}}{\mathrm{n}}$ & $\begin{array}{ll}\pi \\
-1\end{array}$ & $\underset{\sim}{\Omega}$ & $\stackrel{n}{\sim}$ & 号 & 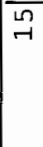 & 吾 & 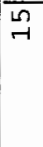 & $\begin{array}{l}n \\
\sim \\
\sim\end{array}$ & \begin{tabular}{|l|}
5 \\
-1
\end{tabular} & $\begin{array}{ll}n \\
-1\end{array}$ & $\stackrel{\Omega}{\Omega}$ & $\begin{array}{ll}n \\
-1\end{array}$ & $\stackrel{\overbrace{}}{\sim}$ & $\stackrel{2}{-1}$ \\
\hline
\end{tabular}

\|

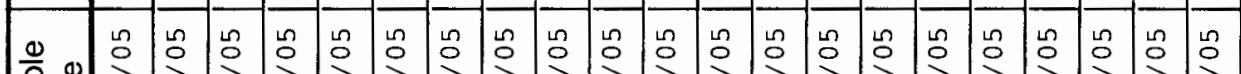

它

嵌

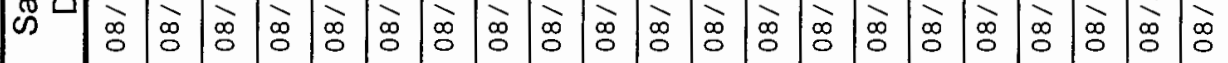

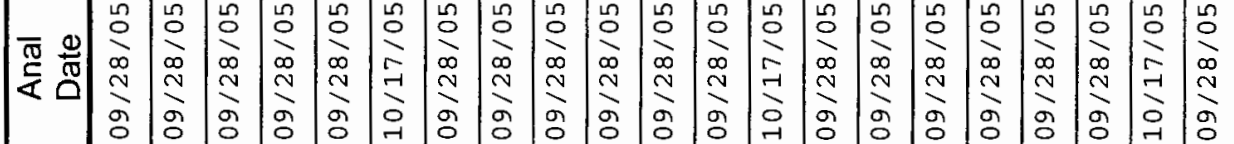

:

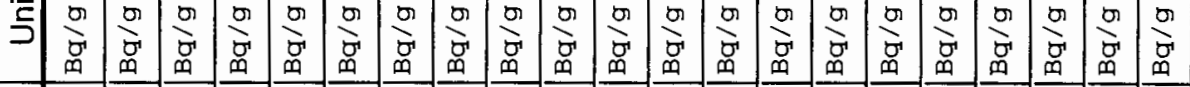

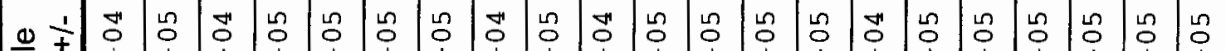

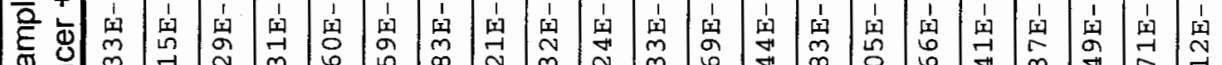

ॠ

文

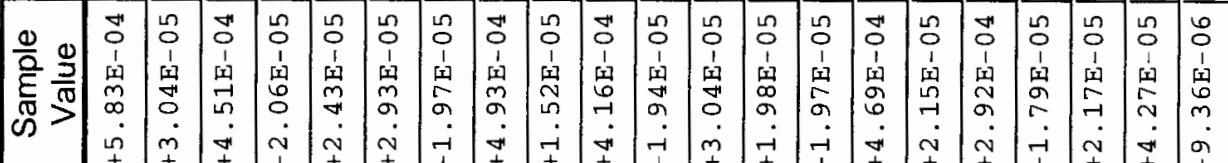

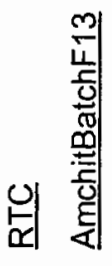

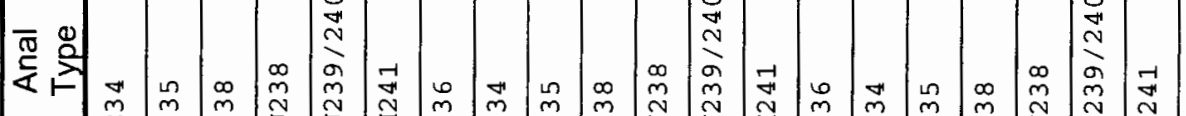

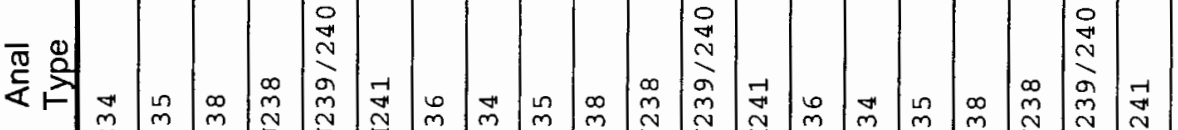

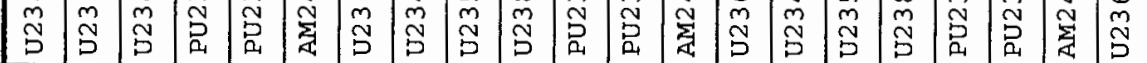

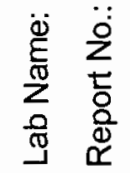

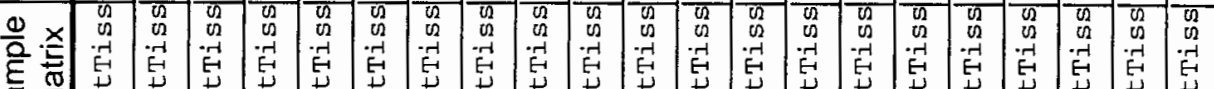

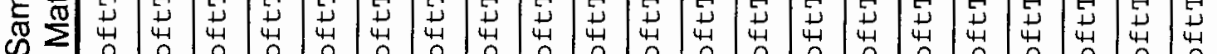

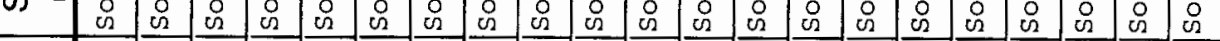




\section{$\frac{1}{20}$}

\begin{tabular}{|c|c|c|c|c|c|c|c|c|c|c|c|c|c|c|c|c|c|c|c|c|c|c|}
\hline $\begin{array}{l}\mathrm{U} \\
\end{array}$ & & & & & & & & & & & & & & & & & & & & & & \\
\hline$\frac{1}{\Sigma}$ & $\begin{array}{l}n \\
0 \\
1 \\
1 \\
91 \\
6 \\
0 \\
-1 \\
+\end{array}$ & $\begin{array}{l}n \\
0 \\
1 \\
1 \\
0 \\
0 \\
? \\
+ \\
+1 \\
+\end{array}$ & $\begin{array}{l}2 \\
0 \\
1 \\
1 \\
9 \\
0 \\
0 \\
\vdots \\
+ \\
+\end{array}$ & 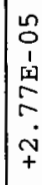 & $\begin{array}{l}n \\
2 \\
1 \\
1 \\
m \\
7 \\
9 \\
7 \\
7\end{array}$ & $\begin{array}{l}12 \\
0 \\
1 \\
5 \\
7 \\
7 \\
7\end{array}$ & & $\begin{array}{l}n \\
0 \\
1 \\
1 \\
\vdots \\
0 \\
0 \\
\dot{m} \\
+ \\
+\end{array}$ & $\begin{array}{l}n \\
0 \\
1 \\
1 \\
1 \\
0 \\
0 \\
0 \\
9 \\
+\end{array}$ & $\begin{array}{l}n \\
0 \\
1 \\
1 \\
5 \\
\sigma \\
\vdots \\
+ \\
+\end{array}$ & $\begin{array}{l}n \\
0 \\
1 \\
1 \\
\text { fy } \\
0 \\
0 \\
0 \\
-1 \\
7\end{array}$ & $\begin{array}{l}n \\
0 \\
0 \\
1 \\
0 \\
9 \\
m \\
m \\
m \\
+\end{array}$ & $\begin{array}{l}n \\
0 \\
1 \\
1 \\
0 \\
0 \\
? \\
0 \\
7\end{array}$ & $\begin{array}{l}2 \\
0 \\
1 \\
1 \\
1 \\
0 \\
0 \\
0 \\
0 \\
+\end{array}$ & 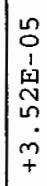 & $\begin{array}{l}2 \\
0 \\
1 \\
1 \\
0 \\
0 \\
0 \\
\sim \\
+ \\
+\end{array}$ & 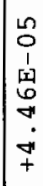 & $\begin{array}{l}n \\
0 \\
1 \\
9 \\
1 \\
2 \\
-1 \\
m \\
m \\
+\end{array}$ & $\begin{array}{l}n \\
0 \\
1 \\
1 \\
0 \\
0 \\
0 \\
i \\
+ \\
+\end{array}$ & 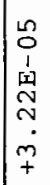 & 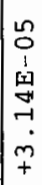 & $\mid \begin{array}{l}0 \\
0 \\
1 \\
1 \\
\text { cl } \\
0 \\
0 \\
0 \\
0 \\
+\end{array}$ \\
\hline 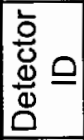 & 5 & 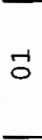 & $\vec{\sigma}$ & $\stackrel{\Perp}{\sim}$ & $\stackrel{4}{-10}$ & $\stackrel{5}{5}$ & & -1 & O & O & I0 & 10 & 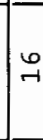 & $\underset{-1}{6}$ & I & on & 10 & $\stackrel{m}{0}$ & of & og & og & 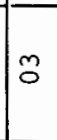 \\
\hline$\frac{E}{\frac{E}{0}} \frac{\overline{0}}{\frac{D}{0}}$ & $\begin{array}{l}n \\
\infty \\
\infty\end{array}$ & $\begin{array}{l}n \\
\infty \\
\infty \\
\sigma\end{array}$ & $\begin{array}{l}n \\
\infty \\
\infty \\
\sigma\end{array}$ & : & ? & 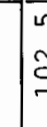 & & $\begin{array}{l}n \\
\infty \\
\infty \\
0\end{array}$ & $\begin{array}{l}2 \\
\vdots \\
\alpha\end{array}$ & $\begin{array}{l}v \\
\dot{a} \\
\sigma\end{array}$ & ì & $\dot{\infty}$ & $\dot{\infty}$ & न & : & $\begin{array}{l}1 \\
\vdots\end{array}$ & $\begin{array}{l}r \\
\dot{0} \\
\sigma\end{array}$ & $\begin{array}{l}2 \\
\dot{0} \\
\sigma\end{array}$ & $\stackrel{\sim}{\sim}$ & $\begin{array}{l}n \\
\dot{n} \\
\infty\end{array}$ & $\begin{array}{l}0 \\
0 \\
0 \\
0 \\
0\end{array}$ & $\begin{array}{l}1 \\
\dot{0} \\
\sigma\end{array}$ \\
\hline & & 5 & 4 & 5 & & 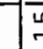 & 4 & $\stackrel{\Omega}{\sim}$ & $\stackrel{\sim}{\rightarrow}$ & -1 & & $\stackrel{n}{n}$ & $\stackrel{\sim}{\sim}$ & $\stackrel{n}{\sim}$ & $\stackrel{5}{-1}$ & $\stackrel{n}{n}$ & $\stackrel{\Omega}{\Omega}$ & 品 & $\stackrel{\square}{\longrightarrow}$ & $\stackrel{\pi}{\sim}$ & $\stackrel{\sim}{\sim}$ & $\stackrel{\Omega}{\sim}$ \\
\hline
\end{tabular}

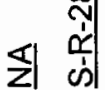

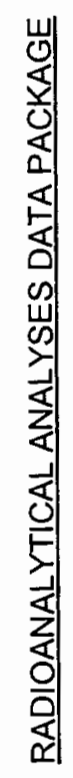

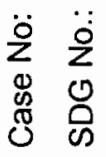

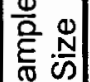

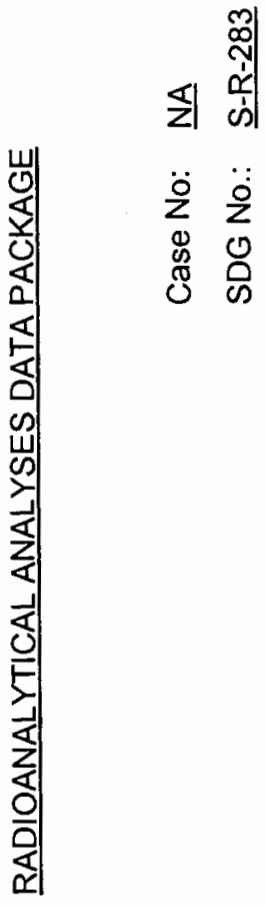

- - - - - - - - - - - - -

ஊ ह⿸厂㔾

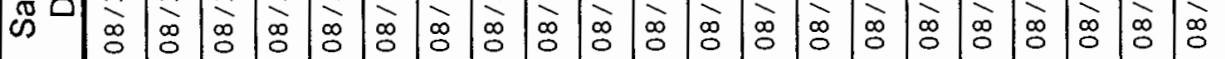

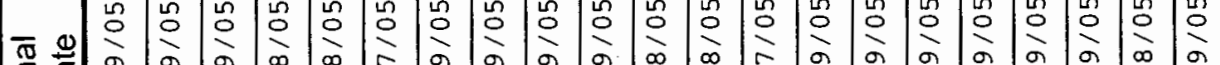

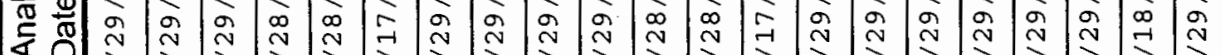

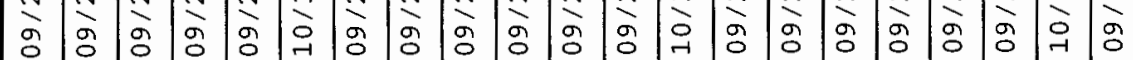

a

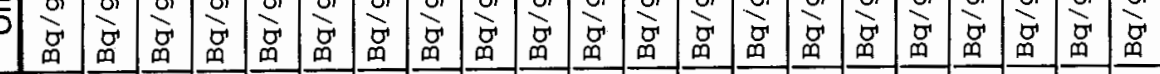

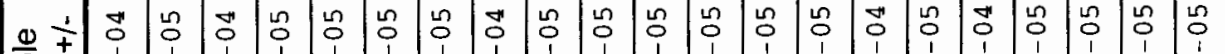

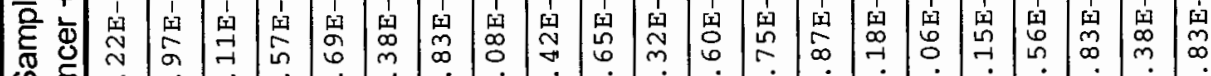
œ

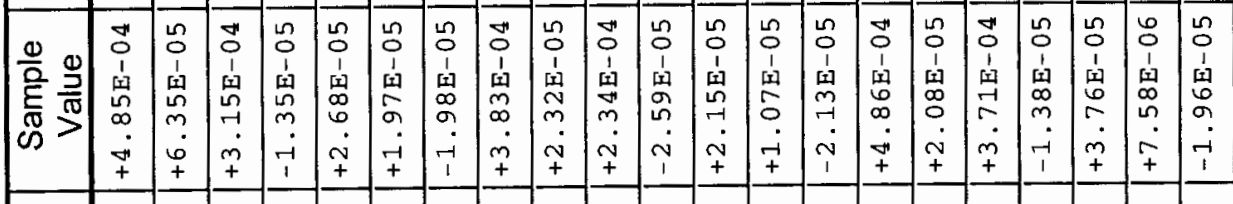

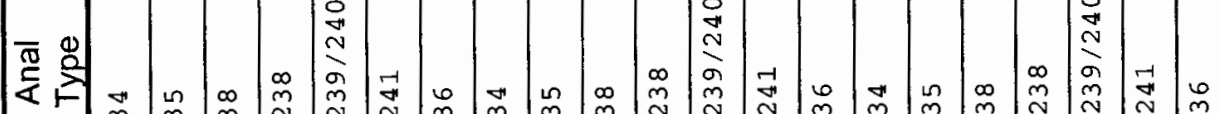

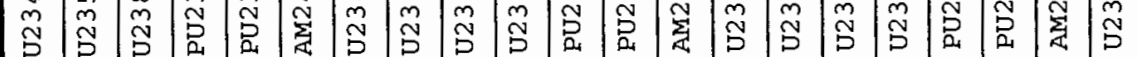

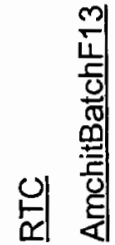

\begin{tabular}{|c|c|c|c|c|c|c|c|c|c|c|c|c|c|c|c|c|c|c|c|c|c|}
\hline 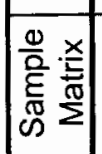 & $\begin{array}{l}1 \\
0 \\
0 \\
0 \\
-11 \\
-1 \\
4 \\
0 \\
0 \\
0\end{array}$ & 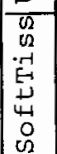 & 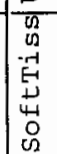 & $\begin{array}{c}0 \\
0 \\
0 \\
-1 \\
-1 \\
\\
4 \\
0 \\
0 \\
0\end{array}$ & 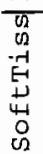 & $\begin{array}{l}0 \\
0 \\
0 \\
-=1 \\
5 \\
4 \\
0 \\
0 \\
0\end{array}$ & 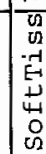 & $\begin{array}{l}0 \\
0 \\
0 \\
-1 \\
-1 \\
4 \\
4 \\
0 \\
0 \\
0\end{array}$ & $\begin{array}{l}0 \\
0 \\
-1 \\
-1 \\
-4 \\
4 \\
0 \\
0 \\
0\end{array}$ & $\begin{array}{l}0 \\
0 \\
0 \\
-11 \\
-1 \\
4 \\
4 \\
0 \\
0 \\
0\end{array}$ & $\begin{array}{l}0 \\
0 \\
0 \\
-1 \\
\\
\\
0 \\
0 \\
0\end{array}$ & 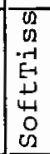 & $\begin{array}{l}0 \\
0 \\
0 \\
-1 \\
-1 \\
4 \\
0 \\
0 \\
0 \\
0\end{array}$ & $\begin{array}{l}n \\
0 \\
0-1 \\
-1 \\
4 \\
4 \\
0 \\
0 \\
2\end{array}$ & $\begin{array}{l}5 \\
0 \\
.2 \\
-1 \\
-1 \\
4 \\
0 \\
0 \\
0 \\
\end{array}$ & $\mid \begin{array}{l}\bar{y} \\
-\pi \\
-\pi\end{array}$ & 10 & \begin{tabular}{|c|}
2 \\
02 \\
-1 \\
-1 \\
-1 \\
4 \\
4 \\
0 \\
0 \\
02 \\
\end{tabular} & \begin{tabular}{|c|c}
$n$ \\
0 \\
0 \\
-1 \\
$E-1$ \\
4 \\
4 \\
0 \\
0 \\
0 \\
0
\end{tabular} & 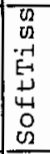 & $\begin{array}{l}0 \\
0 \\
-2 \\
-1 \\
4 \\
4 \\
0 \\
0 \\
0\end{array}$ \\
\hline
\end{tabular}

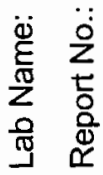

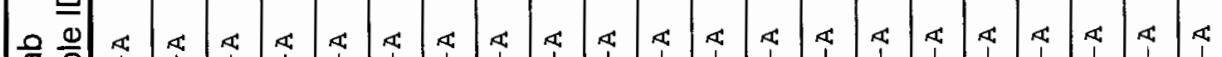

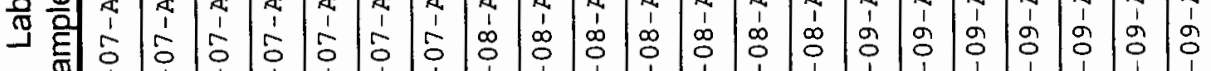

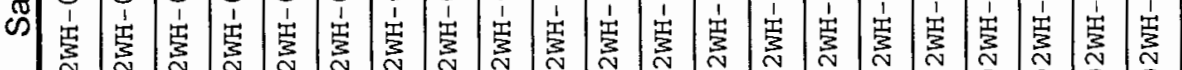
管 
0
0
0
0
0
0
0
0
0
0

\begin{tabular}{|c|c|c|c|c|c|c|c|c|c|c|c|c|c|c|c|c|c|c|c|c|c|c|}
\hline 崩 & & & & & & & & & & & & & & & & & & & & & & \\
\hline 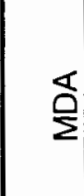 & 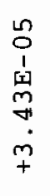 & 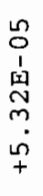 & 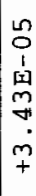 & $\begin{array}{c}n \\
0 \\
1 \\
1 \\
0 \\
0 \\
i \\
0 \\
+ \\
+\end{array}$ & 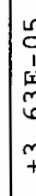 & & 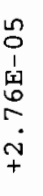 & 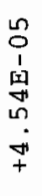 & 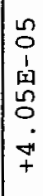 & $\begin{array}{l}2 \\
0 \\
0 \\
1 \\
9 \\
0 \\
0 \\
-1 \\
+\end{array}$ & $\begin{array}{l}8 \\
0 \\
1 \\
1 \\
1 \\
0 \\
6 \\
0 \\
+ \\
+\end{array}$ & 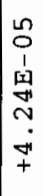 & $\begin{array}{l}\Omega \\
0 \\
1 \\
1 \\
1 \\
0 \\
0 \\
\sim \\
+ \\
+\end{array}$ & 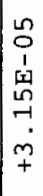 & 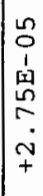 & 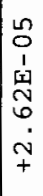 & 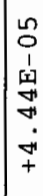 & $\begin{array}{l}n \\
0 \\
0 \\
1 \\
9 \\
7 \\
7 \\
+ \\
+ \\
+\end{array}$ & $\begin{array}{l}n \\
0 \\
1 \\
1 \\
9 \\
m \\
m \\
m \\
+ \\
+\end{array}$ & 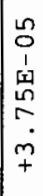 & $\begin{array}{l}n \\
0 \\
1 \\
1 \\
1 \\
0 \\
\infty \\
0 \\
p \\
+\end{array}$ & $\begin{array}{l}2 \\
0 \\
0 \\
1 \\
9 \\
0 \\
0 \\
-1 \\
+\end{array}$ \\
\hline 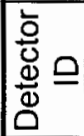 & $\overrightarrow{0}$ & Ot & ठే & 악 & ? & & 足 & $\delta^{*}$ & 告 & | na & $\stackrel{2}{0}$ & $\underset{\sim}{\sim}$ & $\approx$ & $\vec{F}$ & n & 10 & 10 & 10 & F & $\exists$ & I & 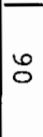 \\
\hline$\frac{5}{0} \stackrel{0}{\frac{5}{0}}$ & 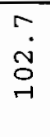 & $\begin{array}{l}\dot{i} \\
\dot{j} \\
\dot{\gamma} \\
-1\end{array}$ & $\dot{i}$ & - & 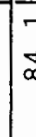 & & $\begin{array}{l}\infty \\
\infty \\
\infty \\
\sigma \\
\sigma\end{array}$ & $\begin{array}{l}\dot{r} \\
\dot{\sigma} \\
\dot{\sigma} \\
-1\end{array}$ & $\begin{array}{l}0 \\
\dot{0} \\
\dot{0} \\
-1\end{array}$ & $\begin{array}{l}0 \\
0 \\
\dot{0} \\
0 \\
-1\end{array}$ & $\begin{array}{l}0 \\
\dot{0} \\
\dot{0} \\
-1\end{array}$ & $\begin{array}{l}\vec{\sigma} \\
\dot{0} \\
\infty\end{array}$ & $\begin{array}{l}\vec{\sigma} \\
\dot{0} \\
\infty\end{array}$ & $\begin{array}{l}\dot{0} \\
\text { के }\end{array}$ & $\begin{array}{l}0 \\
\dot{0} \\
\dot{0} \\
-\end{array}$ & $\begin{array}{l}\infty \\
-1 \\
-1 \\
-1\end{array}$ & $\begin{array}{l}\infty \\
- \\
-1 \\
-1\end{array}$ & $\begin{array}{l}\infty \\
- \\
ت \\
-1\end{array}$ & $\begin{array}{l} \\
0 \\
\infty \\
\infty \\
0\end{array}$ & $\begin{array}{l}6 \\
\dot{m} \\
\infty\end{array}$ & 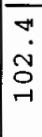 & $\begin{array}{l}\infty \\
- \\
- \\
-1\end{array}$ \\
\hline 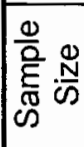 & $\stackrel{\circ}{\sim}$ & $\underset{\sim}{\stackrel{n}{n}}$ & 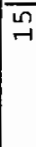 & $\stackrel{n}{n}$ & $\sqrt{5}$ & & $\stackrel{n}{n}$ & $\begin{array}{l}5 \\
-1\end{array}$ & 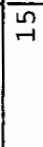 & $\underbrace{5}_{-1}$ & 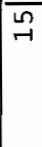 & $\tilde{\sigma}$ & 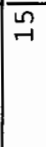 & $\frac{\pi}{7}$ & $\sqrt{\sim}$ & $\begin{array}{ll}\pi \\
-7\end{array}$ & 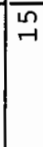 & 吕 & 告 & 号 & $\vec{\sim}$ & 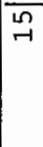 \\
\hline
\end{tabular}

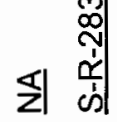

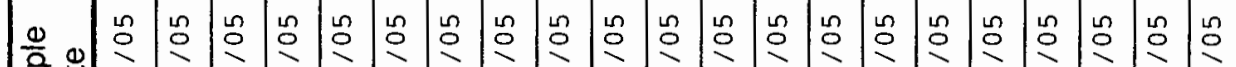

岁

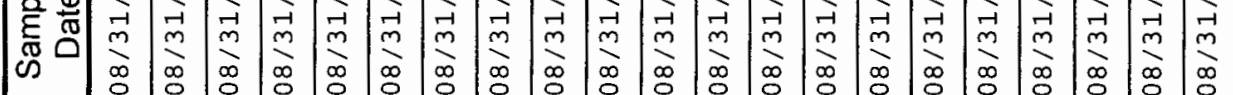

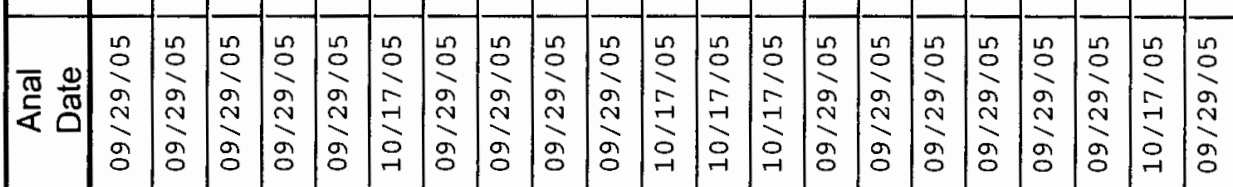

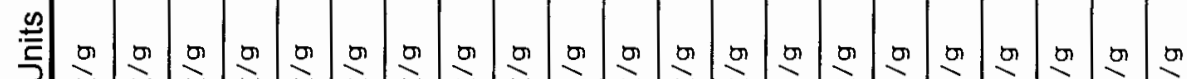

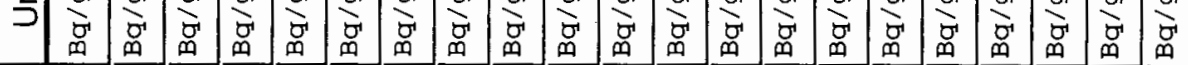

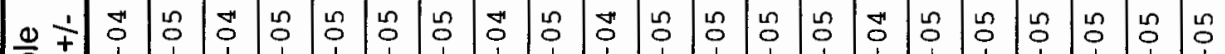
空

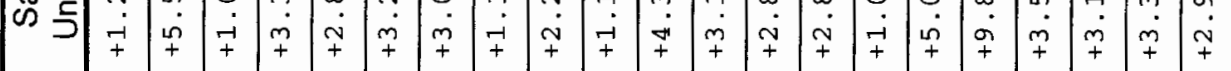

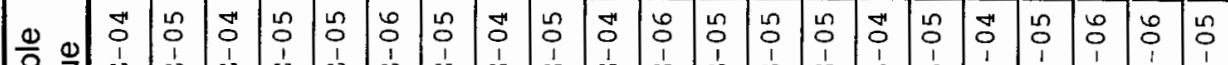

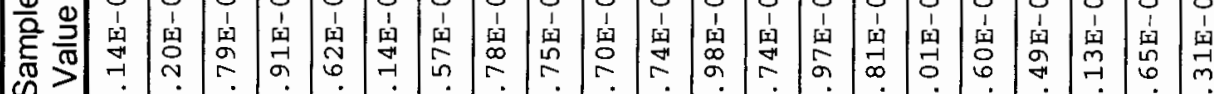

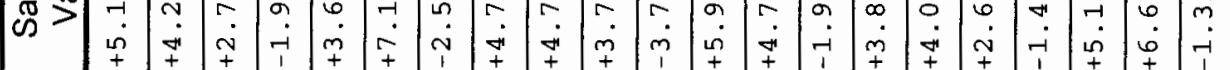

잉

要这

泪

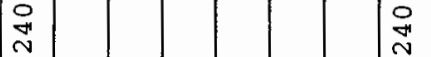

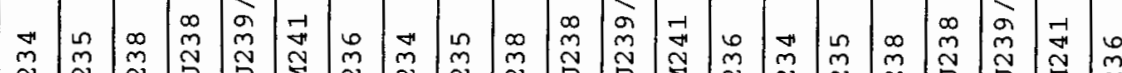

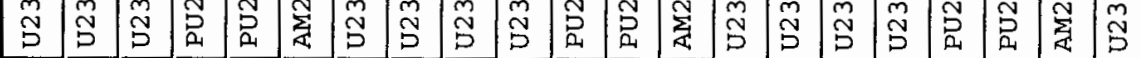

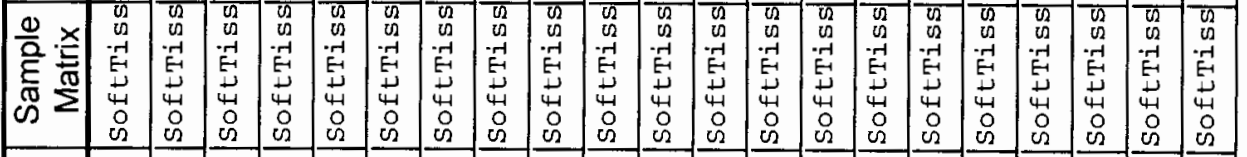

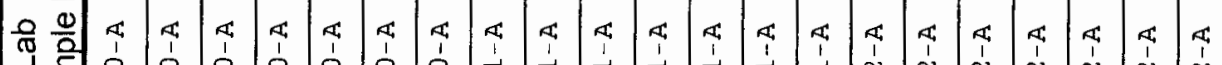

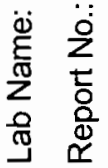

है:

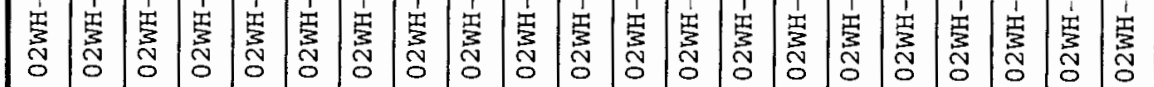

으

$\stackrel{\bar{\Phi}}{\varepsilon}$

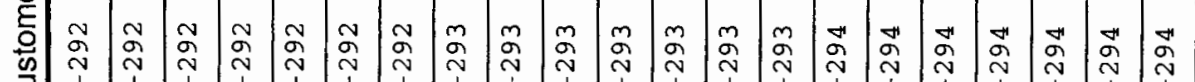

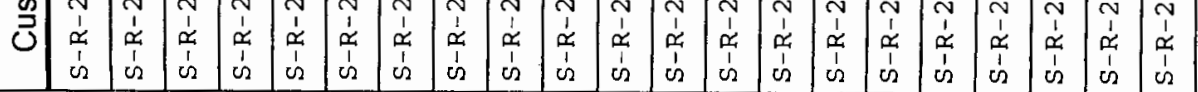




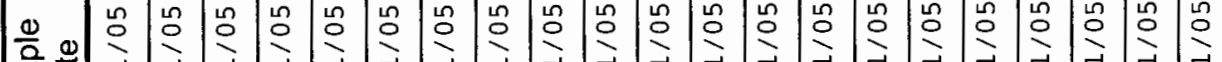

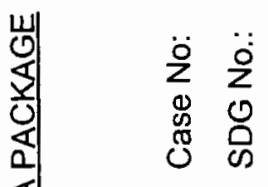

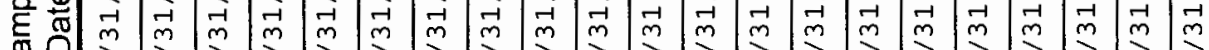

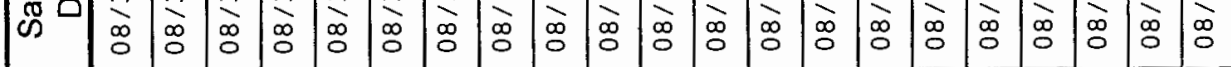

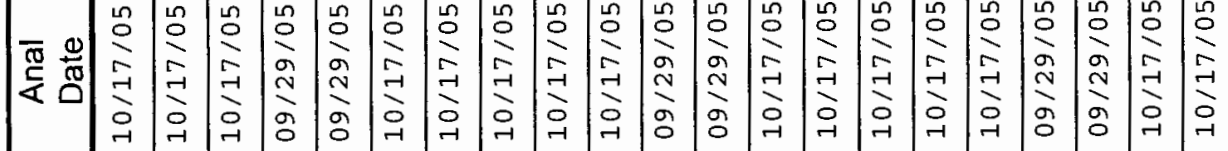
$\therefore$ a a a a a a

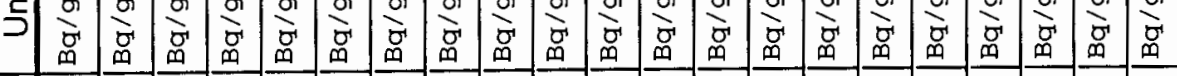
业

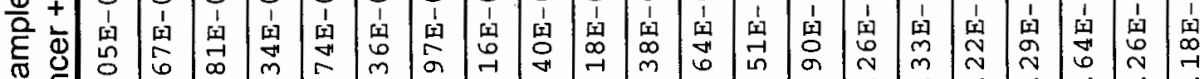

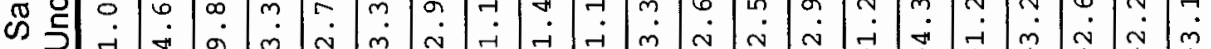
అ

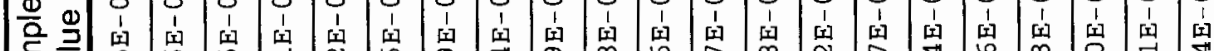

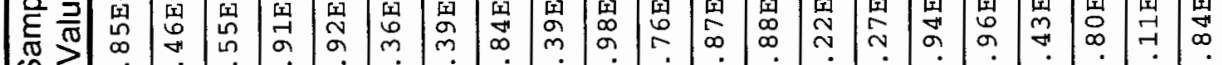

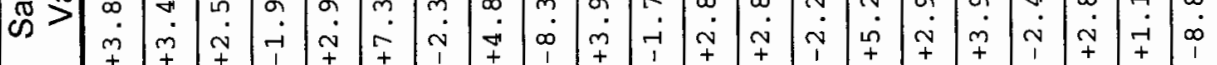

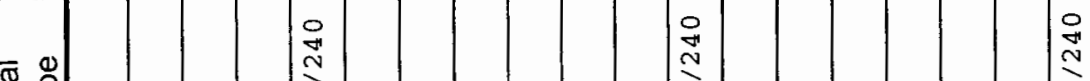

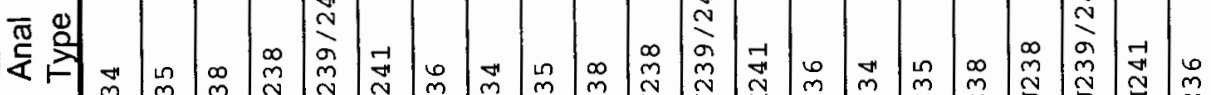

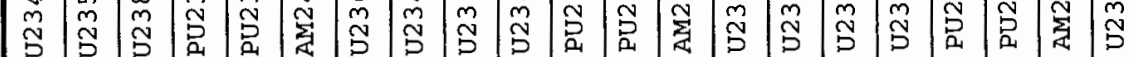

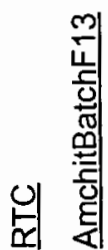

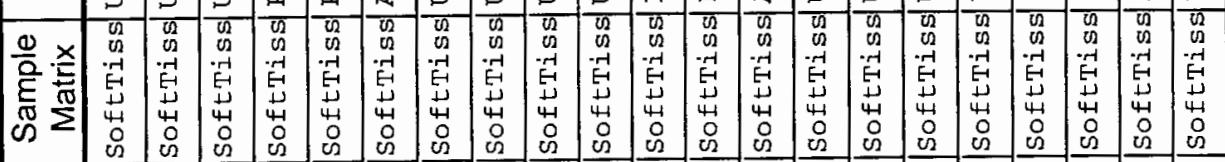

인

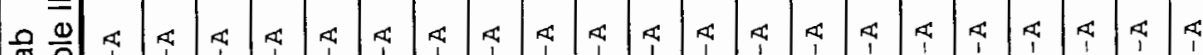

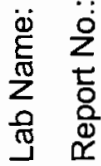

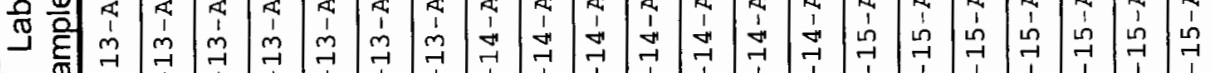
ळ 管 


\begin{tabular}{|c|c|c|c|c|c|c|c|c|c|c|c|c|c|c|c|c|c|c|c|c|c|}
\hline $\begin{array}{l}\mathrm{U} \\
\mathrm{O}\end{array}$ & & & & & & & & & & & & & & & & & & & & & \\
\hline 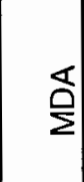 & $\begin{array}{l}n \\
0 \\
1 \\
1 \\
1 \\
\infty \\
\infty \\
+ \\
+ \\
+\end{array}$ & 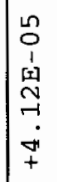 & $\begin{array}{l}n \\
0 \\
1 \\
1 \\
5 \\
\\
r \\
\dot{T} \\
+\end{array}$ & 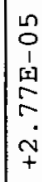 & $\begin{array}{l}n \\
0 \\
1 \\
1 \\
0 \\
0 \\
m \\
7 \\
+ \\
+\end{array}$ & $\begin{array}{l}\text { n } \\
0 \\
1 \\
1 \\
9 \\
0 \\
0 \\
\dot{m} \\
+ \\
+\end{array}$ & 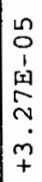 & 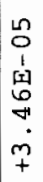 & 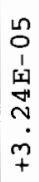 & $\begin{array}{l}n \\
0 \\
1 \\
1 \\
5 \\
0 \\
\dot{m} \\
+\end{array}$ & 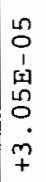 & $\begin{array}{l}n \\
0 \\
1 \\
\text { c1 } \\
m \\
\stackrel{7}{0} \\
m \\
+\end{array}$ & 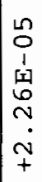 & $\begin{array}{l}n \\
0 \\
1 \\
1 \\
1 \\
5 \\
0 \\
\sim \\
+ \\
+\end{array}$ & \begin{tabular}{l}
$n$ \\
0 \\
1 \\
1 \\
1 \\
0 \\
\multirow{2}{*}{} \\
+ \\
+
\end{tabular} & 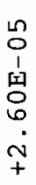 & 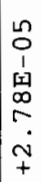 & 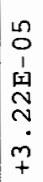 & $\begin{array}{l}n \\
0 \\
1 \\
1 \\
0 \\
0 \\
\dot{m} \\
+\end{array}$ & $\begin{array}{l}\text { n } \\
0 \\
1 \\
1 \\
0 \\
0 \\
\dot{w} \\
+\end{array}$ & 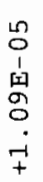 \\
\hline $\begin{array}{l}\overline{\frac{9}{0}} \\
\frac{d}{\Phi} \\
\frac{d}{0}\end{array}$ & $\stackrel{n}{\circ}$ & $\stackrel{2}{\circ}$ & 농 & $\underset{r}{0}$ & 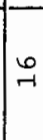 & $\mid \begin{array}{r}6 \\
-1\end{array}$ & 20 & 10 & 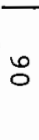 & $\because 8$ & 욱 & 음 & $\stackrel{ }{\sim}$ & $\because$ & so & $\tilde{\sigma}$ & is & $\Rightarrow$ & 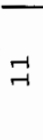 & $\stackrel{\circ}{\rightarrow}$ & s \\
\hline $\begin{array}{l}E \\
\frac{1}{0} \\
\frac{\delta}{0}\end{array}$ & 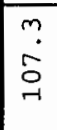 & $\begin{array}{r}m \\
0 \\
0 \\
0 \\
-1\end{array}$ & $\begin{array}{l}m \\
\dot{a} \\
0 \\
-1\end{array}$ & $\overrightarrow{7}$ & $\vec{\infty}$ & $\begin{array}{l}0 \\
\tilde{N} \\
\stackrel{\sim}{\sigma} \\
\end{array}$ & 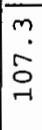 & 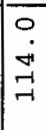 & $\begin{array}{l}0 \\
\dot{J} \\
\vec{\sigma}\end{array}$ & $\begin{array}{l}0 \\
0 \\
\overrightarrow{-} \\
-1\end{array}$ & 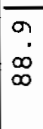 & $\begin{array}{l}a \\
\infty \\
\infty \\
\infty\end{array}$ & $\begin{array}{l}0 \\
0 \\
\dot{-} \\
-1\end{array}$ & $\begin{array}{l}0 \\
\vec{J} \\
-1\end{array}$ & $\begin{array}{l}\sigma \\
\infty \\
\infty \\
\sigma\end{array}$ & $\begin{array}{l}9 \\
0 \\
0 \\
a\end{array}$ & $\begin{array}{l}\tilde{\sigma} \\
\infty \\
\sigma \\
\sigma\end{array}$ & $\begin{array}{l}\vec{r} \\
\dot{1} \\
\infty \\
\infty\end{array}$ & $\begin{array}{l}\vec{y} \\
\dot{\infty} \\
\infty\end{array}$ & $\begin{array}{l}\overrightarrow{1} \\
\dot{\sim} \\
\dot{\sigma}\end{array}$ & $\begin{array}{l}\bar{q} \\
\vdots \\
\infty \\
a\end{array}$ \\
\hline 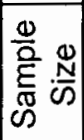 & $\stackrel{\leftrightarrow 2}{\rightarrow-1}$ & $\stackrel{\sim}{\rightarrow}$ & $\stackrel{\sim}{n}$ & $\underset{\sim}{\tilde{\sim}}$ & $\stackrel{5}{\rightarrow}$ & I & $\stackrel{\sim}{\sim}$ & $\stackrel{\Omega}{\rightarrow-}$ & $\stackrel{\leftrightarrow}{\Omega}$ & $\stackrel{\Omega}{-1}$ & $\stackrel{n}{\rightarrow}$ & 吕 & $\bar{\sim}$ & $\operatorname{man}_{-1}$ & $\stackrel{n}{\rightarrow}$ & $\stackrel{\text { ก }}{\rightarrow}$ & 吕 & 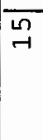 & $\stackrel{\sim}{\sim}$ & $\stackrel{n}{\rightarrow}$ & $\stackrel{\mathrm{n}}{\rightarrow}$ \\
\hline
\end{tabular}

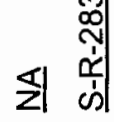

岁

ఋ हृ

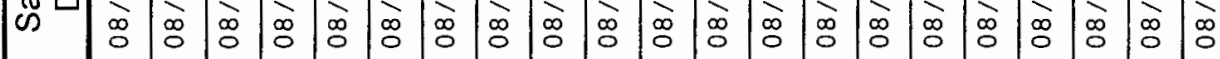

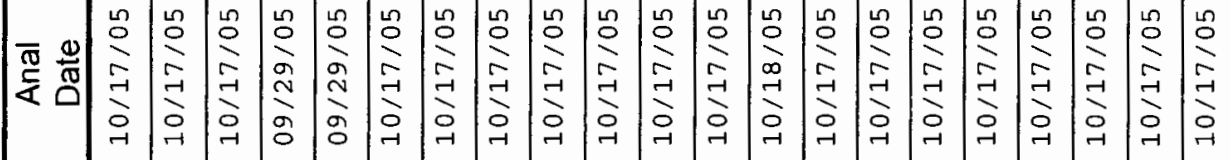
ca

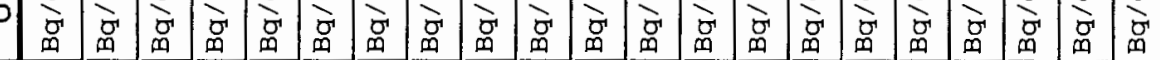
幽南

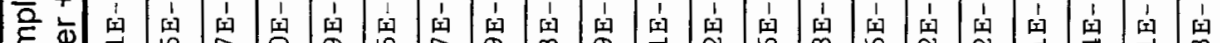

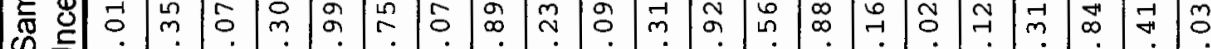

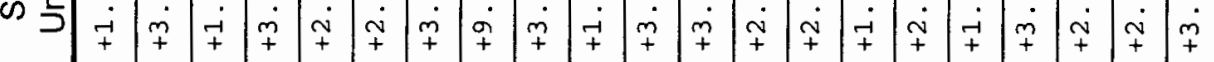

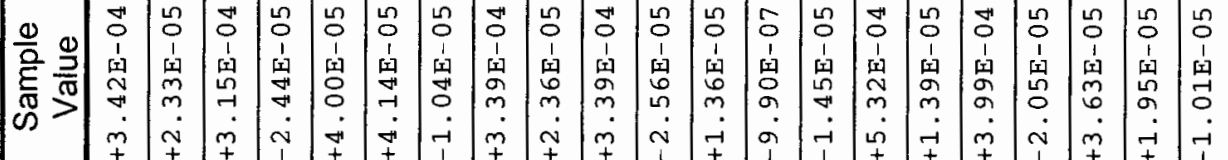
ॠ

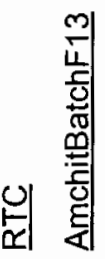

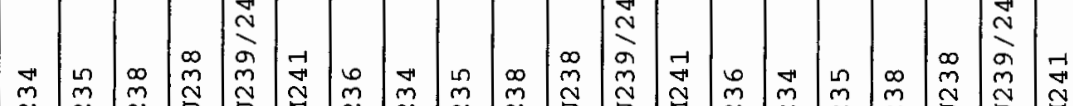

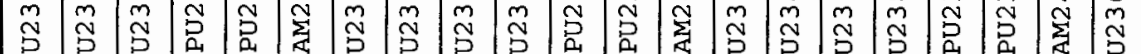

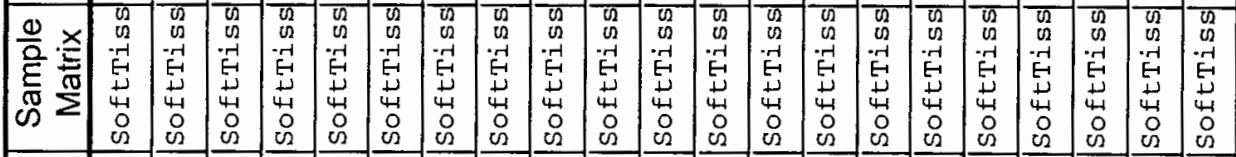

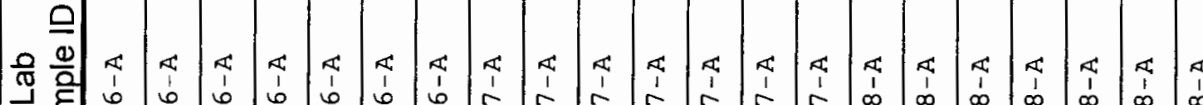

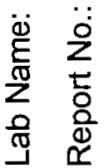
ळึ

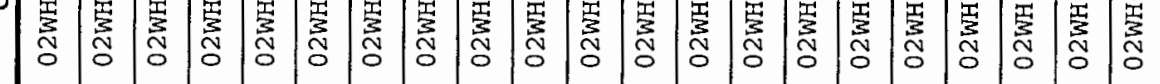




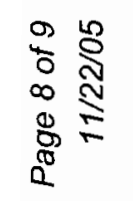

\begin{tabular}{|c|c|c|c|c|c|c|c|c|c|c|c|c|c|c|}
\hline \begin{tabular}{l|l} 
\\
\end{tabular} & & & & & & & & & & & & & & \\
\hline $\begin{array}{l}\nwarrow \\
\vdots\end{array}$ & $\begin{array}{l}n \\
0 \\
1 \\
1 \\
0 \\
o \\
\sim \\
\dot{m} \\
+\end{array}$ & $\begin{array}{l}n \\
0 \\
1 \\
1 \\
9 \\
\sigma \\
\forall \\
\vdots \\
+ \\
+\end{array}$ & $\begin{array}{l}0 \\
1 \\
1 \\
5 \\
5 \\
\vdots \\
+ \\
+\end{array}$ & $\begin{array}{l}n \\
0 \\
1 \\
1 \\
n \\
2 \\
n \\
0 \\
m \\
+ \\
+\end{array}$ & $\mid \begin{array}{l}n \\
0 \\
1 \\
1 \\
0 \\
0 \\
o \\
0 \\
0 \\
+\end{array}$ & $\begin{array}{l}n \\
0 \\
1 \\
1 \\
1 \\
m \\
m \\
0 \\
+ \\
+\end{array}$ & 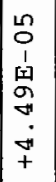 & 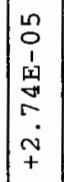 & $\begin{array}{c}n \\
0 \\
1 \\
1 \\
1 \\
5 \\
n \\
\sim \\
+ \\
+\end{array}$ & 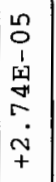 & \begin{tabular}{|l|}
$n$ \\
0 \\
1 \\
1 \\
1 \\
0 \\
0 \\
0 \\
0 \\
+ \\
\end{tabular} & 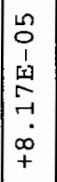 & 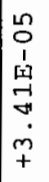 & $\begin{array}{l}n \\
0 \\
1 \\
1 \\
1 \\
0 \\
0 \\
0 \\
\dot{z} \\
+\end{array}$ \\
\hline 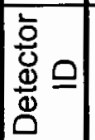 & $\stackrel{\infty}{\infty}$ & $\left.\right|_{0} ^{\infty}$ & 番 & $\Im$ & テ & 10 & ${ }_{0}^{\infty}$ & 10 & 5 & 5 & $\stackrel{\infty}{\circ}$ & ${ }_{0}^{\infty}$ & $\overrightarrow{-1}$ & 5 \\
\hline 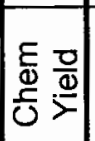 & $\begin{array}{c}m \\
\dot{m} \\
\sigma\end{array}$ & $\begin{array}{l}\dot{m} \\
\dot{\sigma}\end{array}$ & $\begin{array}{l}m \\
\dot{m}\end{array}$ & $\begin{array}{l}-1 \\
0 \\
0\end{array}$ & $\begin{array}{l}\overrightarrow{1} \\
\dot{0}\end{array}$ & $\begin{array}{l}\dot{g} \\
\dot{r} \\
\vec{r}\end{array}$ & $\begin{array}{l}m \\
\text { an } \\
\sigma\end{array}$ & $\begin{array}{l}m \\
\dot{v} \\
\dot{v}\end{array}$ & $\begin{array}{l}m \\
\dot{y} \\
\exists\end{array}$ & $\begin{array}{l}m \\
\dot{y} \\
\mathcal{y}\end{array}$ & $\begin{array}{l}7 \\
\dot{\sigma} \\
\sigma\end{array}$ & $\begin{array}{l}-1 \\
\dot{a} \\
a\end{array}$ & $\begin{array}{l}m \\
\dot{m} \\
\tilde{o}\end{array}$ & $\begin{array}{l}m \\
\dot{j} \\
\vec{I}\end{array}$ \\
\hline 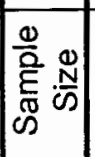 & \begin{tabular}{|l|}
$\ddot{H}$ \\
$\ddot{H}$
\end{tabular} & 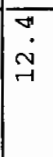 & 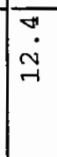 & $\begin{array}{l}\vec{\vdots} \\
\stackrel{\sim}{*}\end{array}$ & \begin{tabular}{|l|}
$\vec{n}$ \\
$\stackrel{\sim}{*}$
\end{tabular} & \begin{tabular}{l|}
$\overrightarrow{+}$ \\
$\stackrel{\sim}{\sim}$
\end{tabular} & \begin{tabular}{|l|}
$\vec{n}$ \\
$\stackrel{\vec{H}}{ }$
\end{tabular} & \begin{tabular}{|l|} 
\\
$m$ \\
$\vec{H}$
\end{tabular} & \begin{tabular}{|l|}
$\vec{y}$ \\
$\dot{m}$
\end{tabular} & \begin{tabular}{|l|}
$\vec{n}$ \\
$\dot{m}$ \\
$\vec{g}$
\end{tabular} & $\begin{array}{l}\vec{\sigma} \\
\dot{m}\end{array}$ & \begin{tabular}{|l|}
$\dot{m}$ \\
$\dot{\vec{n}}$
\end{tabular} & $\overrightarrow{\ddot{n}}$ & 票 \\
\hline 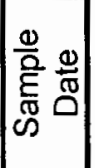 & $\begin{array}{l}n \\
\stackrel{0}{0} \\
\stackrel{-}{\infty} \\
\infty \\
\infty \\
0\end{array}$ & 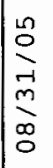 & 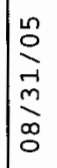 & $\mid \begin{array}{l}n \\
0 \\
\\
m \\
\infty \\
0 \\
0\end{array}$ & $\mid \begin{array}{l}\stackrel{n}{0} \\
0 \\
-1 \\
m \\
\infty \\
0 \\
0\end{array}$ & $\mid \begin{array}{c}n \\
0 \\
2 \\
m \\
\infty \\
0 \\
0\end{array}$ & $\mid \begin{array}{c}n \\
0 \\
-1 \\
m \\
\infty \\
0 \\
0\end{array}$ & $\mid \begin{array}{c}n \\
0 \\
-1 \\
m \\
\infty \\
\infty \\
0\end{array}$ & $\mid \begin{array}{c}n \\
0 \\
2 \\
m \\
\infty \\
0 \\
0\end{array}$ & $\begin{array}{l}n \\
0 \\
0 \\
m \\
m \\
\infty \\
0 \\
0\end{array}$ & $\mid \begin{array}{l}2 \\
0 \\
2 \\
m \\
0 \\
0 \\
o\end{array}$ & $\mid \begin{array}{l}n \\
\stackrel{n}{2} \\
\stackrel{2}{m} \\
\infty \\
0 \\
0\end{array}$ & $\begin{array}{l}n \\
0 \\
\\
m \\
\infty \\
0 \\
0\end{array}$ & $\begin{array}{l}n \\
\stackrel{2}{0} \\
- \\
0 \\
0 \\
\infty \\
0\end{array}$ \\
\hline $\overrightarrow{0}$ & $\begin{array}{l}\stackrel{n}{2} \\
\stackrel{2}{2} \\
\text { न-1 } \\
0 \\
0 \\
-1\end{array}$ & 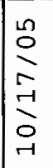 & 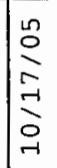 & 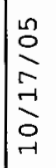 & $\begin{array}{l}\stackrel{n}{0} \\
\stackrel{2}{2} \\
\\
0 \\
0 \\
-1\end{array}$ & 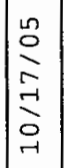 & 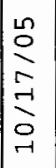 & $\begin{array}{l}\stackrel{n}{0} \\
0 \\
\infty \\
\stackrel{2}{\sim} \\
0 \\
\sim\end{array}$ & 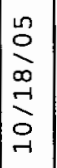 & 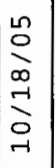 & 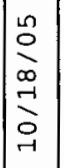 & 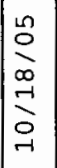 & $\begin{array}{l}n \\
0 \\
0 \\
0 \\
-1 \\
0 \\
0 \\
-1\end{array}$ & 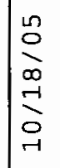 \\
\hline & $\frac{\sigma}{\sigma}$ & $\frac{\sigma}{0}$ & $\begin{array}{l}\sigma \\
\sigma \\
\sigma \\
\end{array}$ & $\frac{0}{9}$ & 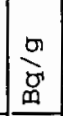 & \begin{tabular}{|l|}
0 \\
$\partial^{\prime}$ \\
\end{tabular} & 皇 & 品 & $\begin{array}{l}0 \\
0 \\
0 \\
\end{array}$ & $\begin{array}{l}0 \\
0 \\
0 \\
\end{array}$ & $\begin{array}{l}5 \\
9 \\
1\end{array}$ & 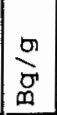 & 吕 & \begin{tabular}{|l}
0 \\
$g$ \\
$g$ \\
9
\end{tabular} \\
\hline 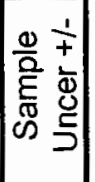 & $\begin{array}{l}m \\
0 \\
1 \\
1 \\
5 \\
0 \\
\llcorner \\
-1 \\
+\end{array}$ & $\begin{array}{l}\overrightarrow{0} \\
0 \\
1 \\
\omega \\
\omega \\
m \\
m \\
\overrightarrow{7} \\
7\end{array}$ & 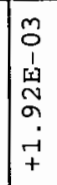 & $\begin{array}{l}n \\
0 \\
1 \\
1 \\
9 \\
0 \\
0 \\
0 \\
+\end{array}$ & 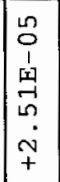 & 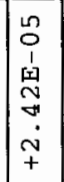 & \begin{tabular}{|l|} 
\\
0 \\
1 \\
1 \\
1 \\
$m$ \\
$m$ \\
7 \\
+ \\
+
\end{tabular} & $\begin{array}{l}0 \\
0 \\
1 \\
1 \\
\mathfrak{1} \\
0 \\
0 \\
0 \\
+\end{array}$ & $\begin{array}{l}n \\
0 \\
1 \\
1 \\
9 \\
0 \\
\forall \\
0 \\
+ \\
+\end{array}$ & $\begin{array}{c}m \\
0 \\
1 \\
y_{1} \\
0 \\
0 \\
\dot{j} \\
+\end{array}$ & 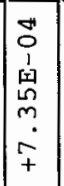 & $\begin{array}{l}\overrightarrow{0} \\
1 \\
1 \\
0 \\
0 \\
+1 \\
0 \\
+ \\
\end{array}$ & \begin{tabular}{|l|}
$n$ \\
0 \\
1 \\
1 \\
\\
$\infty$ \\
0 \\
$\sim$ \\
+ \\
+ \\
\end{tabular} & $\begin{array}{l}1 \\
\stackrel{9}{9} \\
\text { r } \\
\dot{m} \\
+\end{array}$ \\
\hline 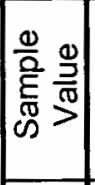 & 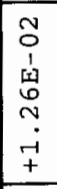 & $\begin{array}{l}\overrightarrow{0} \\
1 \\
1 \\
9 \\
0 \\
\vdots \\
10 \\
+ \\
\end{array}$ & 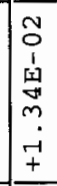 & $\begin{array}{l}2 \\
0 \\
1 \\
1 \\
1 \\
0 \\
0 \\
0 \\
1 \\
1 \\
1\end{array}$ & $\begin{array}{c}0 \\
0 \\
1 \\
1 \\
0 \\
0 \\
0 \\
\infty \\
1 \\
1 \\
\end{array}$ & \begin{tabular}{|c|} 
\\
0 \\
1 \\
1 \\
$\sigma$ \\
$\sigma$ \\
-1 \\
1 \\
\end{tabular} & \begin{tabular}{|l|}
0 \\
0 \\
1 \\
1 \\
0 \\
0 \\
0 \\
1 \\
\end{tabular} & $\begin{array}{l}0 \\
0 \\
1 \\
1 \\
\vdots \\
\vdots \\
0 \\
0 \\
+ \\
\end{array}$ & 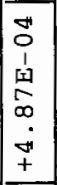 & \begin{tabular}{|l|}
$m$ \\
0 \\
1 \\
$y$ \\
$\{1$ \\
$\vec{y}$ \\
0 \\
0 \\
+ \\
\end{tabular} & \begin{tabular}{|l|}
$m$ \\
0 \\
1 \\
1 \\
1 \\
+5 \\
0 \\
0 \\
+ \\
\end{tabular} & \begin{tabular}{|l|}
$m$ \\
0 \\
1 \\
1 \\
0 \\
0 \\
$n$ \\
0 \\
+ \\
\end{tabular} & 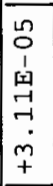 & 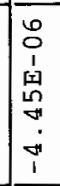 \\
\hline एँ & 岕 & $\stackrel{\stackrel{n}{n}}{\stackrel{\sim}{S}}$ & 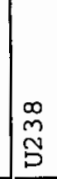 & 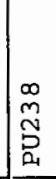 & 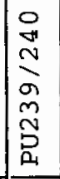 & $\begin{array}{l}\vec{a} \\
\vec{J} \\
\widetilde{a}\end{array}$ & $\begin{array}{l}0 \\
\tilde{N} \\
\tilde{S}\end{array}$ & 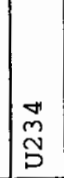 & 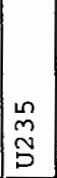 & \begin{tabular}{|l|}
$\infty$ \\
$\tilde{N}$ \\
$\mathcal{D}$ \\
\end{tabular} & 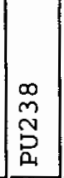 & 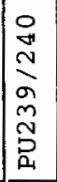 & 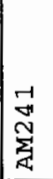 & 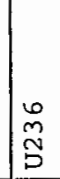 \\
\hline 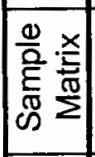 & \begin{tabular}{|l|}
0 \\
0 \\
0 \\
-4 \\
4 \\
4 \\
0 \\
0 \\
0 \\
\end{tabular} & $\begin{array}{l}0 \\
0 \\
0-1 \\
-1 \\
-1 \\
\end{array}$ & 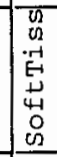 & $\begin{array}{c}0 \\
0 \\
0 \\
-1 \\
-1 \\
4 \\
4 \\
0 \\
0 \\
02 \\
0\end{array}$ & \begin{tabular}{|l}
0 \\
0 \\
0 \\
-4 \\
$E$ \\
4 \\
0 \\
0 \\
0 \\
\end{tabular} & \begin{tabular}{|l|}
$n$ \\
0 \\
-4 \\
-4 \\
4 \\
0 \\
0 \\
0 \\
\end{tabular} & 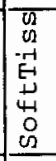 & \begin{tabular}{|c|} 
\\
0 \\
-1 \\
-1 \\
-1 \\
4 \\
0 \\
0 \\
02 \\
\end{tabular} & \begin{tabular}{|l|}
$n$ \\
0 \\
0 \\
-1 \\
4 \\
4 \\
0 \\
0 \\
02 \\
\end{tabular} & \begin{tabular}{|c|} 
\\
0 \\
0 \\
-1 \\
$E$ \\
\\
0 \\
0 \\
0 \\
\end{tabular} & \begin{tabular}{|l|} 
\\
0 \\
0 \\
-4 \\
\\
4 \\
0 \\
0 \\
02 \\
\end{tabular} & \begin{tabular}{|c|} 
\\
0 \\
0 \\
-1 \\
-1 \\
4 \\
4 \\
0 \\
0 \\
\end{tabular} & $\begin{array}{l}0 \\
0 \\
0-1 \\
-1 \\
5 \\
4 \\
0 \\
0 \\
02 \\
02\end{array}$ & 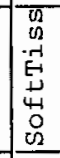 \\
\hline 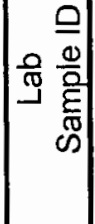 & 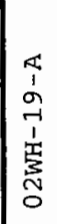 & 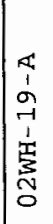 & $\begin{array}{l}4 \\
1 \\
\vdots \\
7 \\
7 \\
1 \\
3 \\
3\end{array}$ & 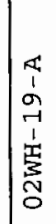 & 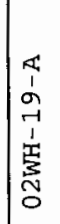 & 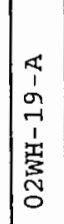 & 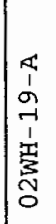 & 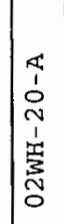 & 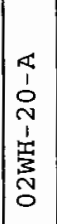 & 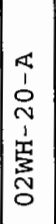 & 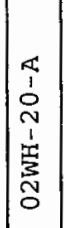 & 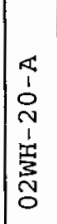 & 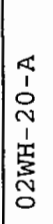 & 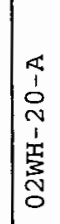 \\
\hline | & $\begin{array}{l}0 \\
m \\
1 \\
\text { s. }\end{array}$ & $\mid \begin{array}{l}1 \\
0 \\
0 \\
1 \\
1 \\
1 \\
1 \\
v 2\end{array}$ & $\begin{array}{l}m \\
1 \\
w \\
w \\
c \\
c \\
\end{array}$ & $\begin{array}{l}0 \\
m \\
1 \\
1 \\
1 \\
1 \\
0 \\
0\end{array}$ & 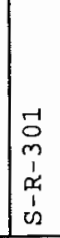 & $\mid \begin{array}{l}-1 \\
0 \\
0 \\
1 \\
x \\
1 \\
1 \\
0\end{array}$ & \begin{tabular}{|l}
-1 \\
0 \\
0 \\
1 \\
1 \\
1 \\
1 \\
1 \\
02 \\
\end{tabular} & $\mid \begin{array}{l}0 \\
0 \\
0 \\
1 \\
1 \\
2 \\
1 \\
v \\
\end{array}$ & \begin{tabular}{|c|} 
\\
0 \\
0 \\
1 \\
0 \\
1 \\
1 \\
2 \\
\end{tabular} & $\mid \begin{array}{c}0 \\
0 \\
0 \\
1 \\
x \\
1 \\
0 \\
0\end{array}$ & $\mid \begin{array}{l}N \\
0 \\
0 \\
1 \\
\alpha \\
1 \\
0 \\
\end{array}$ & $\begin{array}{c}0 \\
0 \\
0 \\
1 \\
\vdots \\
1 \\
1 \\
0\end{array}$ & 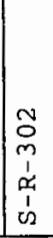 & $\mid \begin{array}{l}\tilde{D} \\
\tilde{D} \\
\tilde{m} \\
\tilde{n}\end{array}$ \\
\hline
\end{tabular}

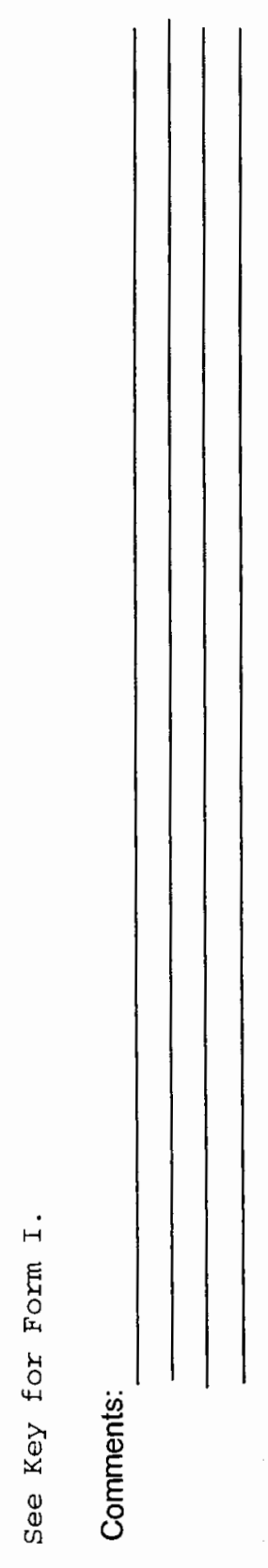


$\begin{array}{ll}0 & 0 \\ 0 & 8 \\ 0 & \mathbb{N} \\ 0 & \mathbb{N} \\ 0 & 5\end{array}$

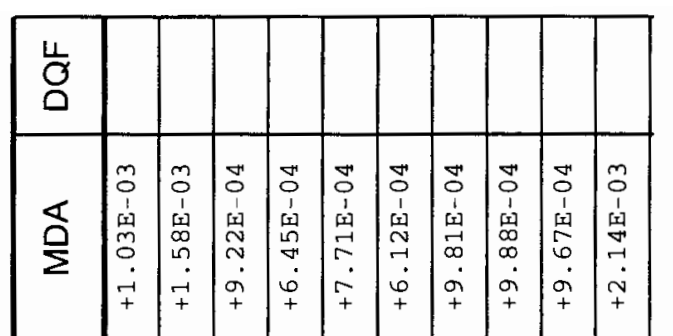

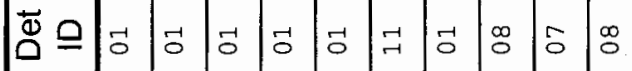

క|

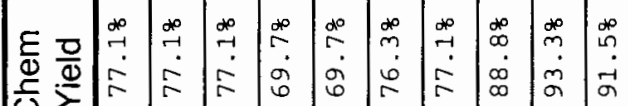

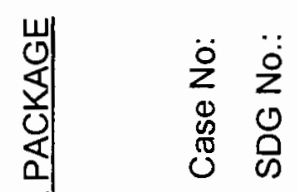

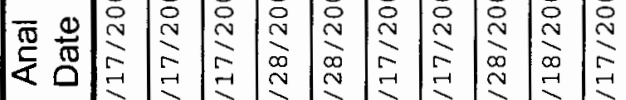

น ก 2 ก

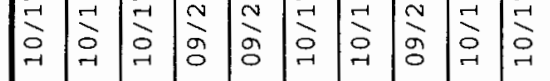

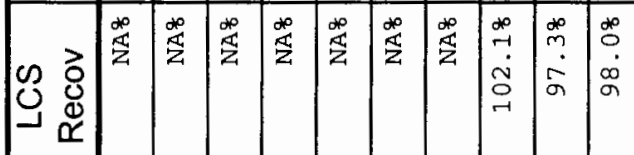

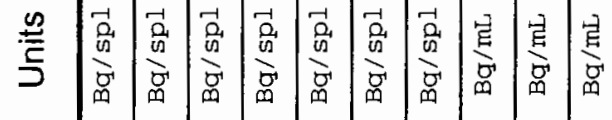

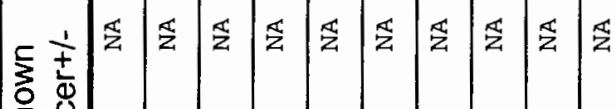

文

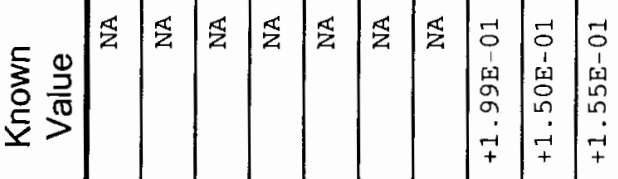

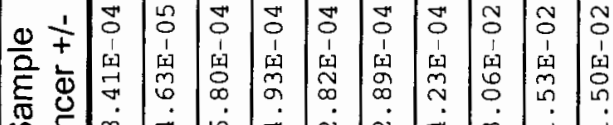

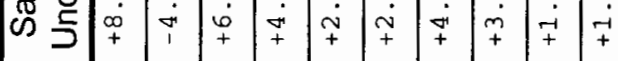

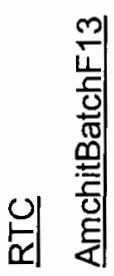

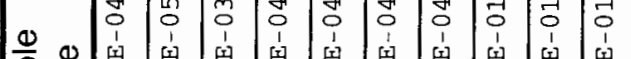

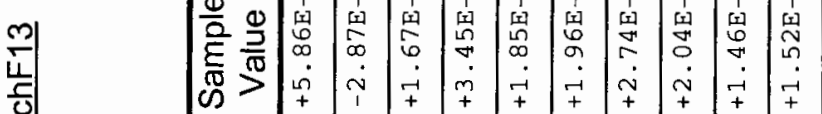

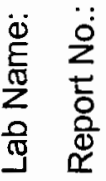

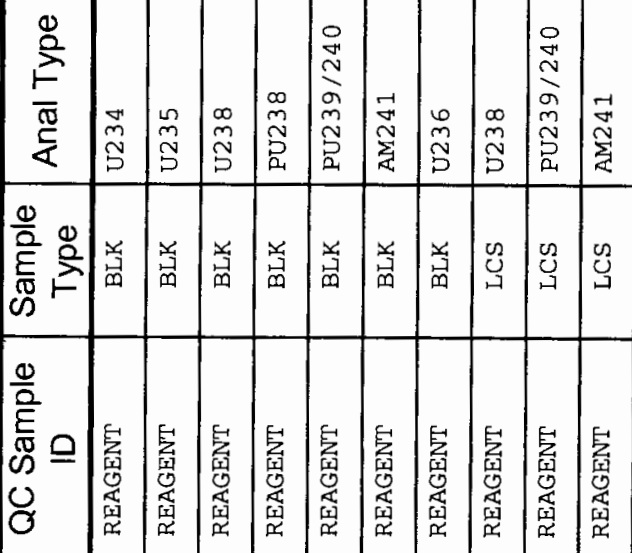



Project: $\quad$ Alpha Analysis for Amchitka Island (Batch 13)

Laboratory: RTC

Report \#: AmchitBatchF13

SDG\#: $\quad$ S-R-283

Summary of 2 and 3 sigma activities

Below are the results for U234, U235, U236, U238, Pu238, Pu239/240, and Am241 for Batch 13 from the Amchitka Island Project that had a result/uncertainty ratio of 2 or more (uncertainty @ one sigma).

\begin{tabular}{|c|c|c|c|c|c|c|}
\hline $\begin{array}{l}\text { Customer } \\
\text { ID }\end{array}$ & Lab ID & Isotope & $\begin{array}{l}\text { Result } \\
\text { Bq/g }\end{array}$ & $\begin{array}{c}\text { Uncertainty } \\
\mathbf{B q} / \mathbf{g}\end{array}$ & MDA & $\begin{array}{c}\text { Result/ } \\
\text { Uncertainty }\end{array}$ \\
\hline S-R-283 & 02WH-01-A & U234 & $3.34 \mathrm{E}-04$ & $9.83 \mathrm{E}-05$ & $3.10 E-05$ & 3.4 \\
\hline S-R-283 & 02WH-01-A & U238 & 2.99E-04 & $1.03 \mathrm{E}-04$ & 2.60E-05 & 2.9 \\
\hline S-R-284 & 02WH-02-A & U234 & 3.27E-04 & $9.64 \mathrm{E}-05$ & 2.96E-05 & 3.4 \\
\hline S-R-284 & 02WH-02-A & U238 & 2.50E-04 & $9.51 E-05$ & 2.96E-05 & 2.6 \\
\hline S-R-285 & 02WH-03-A & U234 & 3.43E-04 & $9.99 E-05$ & 3.18E-05 & 3.4 \\
\hline S-R-285 & 02WH-03-A & U238 & 3.07E-04 & $1.05 E-04$ & 3.18E-05 & 2.9 \\
\hline S-R-286 & 02WH-04-A & U234 & 5.83E-04 & 1.33E-04 & 4.07E-05 & 4.4 \\
\hline S-R-286 & 02WH-04-A & U238 & $4.51 \mathrm{E}-04$ & $1.29 \mathrm{E}-04$ & 4.07E-05 & 3.5 \\
\hline S-R-287 & 02WH-05-A & U234 & 4.93E-04 & $1.21 E-04$ & 2.81E-05 & 4.1 \\
\hline S-R-287 & 02WH-05-A & U238 & $4.16 \mathrm{E}-04$ & $1.24 \mathrm{E}-04$ & 4.13E-05 & 3.4 \\
\hline S-R-288 & 02WH-06-A & U234 & 4.69E-04 & $1.05 E-04$ & $9.78 E-06$ & 4.5 \\
\hline S-R-288 & 02WH-06-A & U238 & 2.92E-04 & $9.41 \mathrm{E}-05$ & $9.78 E-06$ & 3.1 \\
\hline S-R-289 & 02WH-07-A & U234 & 4.85E-04 & 1.22E-04 & $1.61 E-05$ & 4.0 \\
\hline S-R-289 & 02WH-07-A & U235 & 6.35E-05 & 2.97E-05 & 4.58E-05 & 2.1 \\
\hline S-R-289 & 02WH-07-A & U238 & 3.15E-04 & $1.11 \mathrm{E}-04$ & 4.81E-05 & 2.8 \\
\hline S-R-290 & 02WH-08-A & U234 & 3.83E-04 & $1.08 \mathrm{E}-04$ & $3.96 \mathrm{E}-05$ & 3.5 \\
\hline S-R-290 & $02 W H-08-A$ & U238 & $2.34 \mathrm{E}-04$ & $9.65 E-05$ & 1.56E-05 & 2.4 \\
\hline S-R-291 & 02WH-09-A & U234 & 4.86E-04 & 1.18E-04 & $2.64 \mathrm{E}-05$ & 4.1 \\
\hline S-R-291 & 02WH-09-A & U238 & 3.71E-04 & $1.15 \mathrm{E}-04$ & $3.15 \mathrm{E}-05$ & 3.2 \\
\hline S-R-292 & $02 W H-10-A$ & U234 & 5.14E-04 & $1.25 \mathrm{E}-04$ & 3.43E-05 & 4.1 \\
\hline S-R-292 & $02 W H-10-A$ & U238 & 2.79E-04 & $1.03 E-04$ & $3.43 \mathrm{E}-05$ & 2.7 \\
\hline S-R-293 & $02 W H-11-A$ & U234 & $4.78 \mathrm{E}-04$ & 1.19E-04 & 4.05E-05 & 4.0 \\
\hline S-R-293 & $02 W H-11-A$ & U235 & 4.75E-05 & $2.29 \mathrm{E}-05$ & $1.84 \mathrm{E}-05$ & 2.1 \\
\hline S-R-293 & $02 W H-11-A$ & U238 & $3.70 \mathrm{E}-04$ & $1.17 \mathrm{E}-04$ & 4.63E-05 & 3.2 \\
\hline
\end{tabular}




\section{Alpha Analysis for Amchitka Island (Batch 13) Summary of 2 and 3 sigma activities}

page 2

\begin{tabular}{|c|c|c|c|c|c|c|}
\hline $\begin{array}{l}\text { Customer } \\
\text { ID }\end{array}$ & Lab ID & Isotope & $\begin{array}{c}\text { Result } \\
\mathrm{Bq} / \mathrm{g}\end{array}$ & $\begin{array}{c}\text { Uncertainty } \\
\mathrm{Bq} / \mathrm{g}\end{array}$ & MDA & $\begin{array}{c}\text { Result/ } \\
\text { Uncertainty }\end{array}$ \\
\hline S-R-294 & $02 \mathrm{WH}-12-\mathrm{A}$ & U234 & 3.81E-04 & $1.04 \mathrm{E}-04$ & 2.62E-05 & 3.7 \\
\hline S-R-294 & $02 W H-12-A$ & U238 & $2.60 E-04$ & $9.88 \mathrm{E}-05$ & 4.41E-05 & 2.6 \\
\hline S-R-295 & $02 W H-13-A$ & U234 & 3.85E-04 & $1.05 E-04$ & 3.63E-05 & 3.7 \\
\hline S-R-295 & $02 W H-13-A$ & U238 & $2.55 E-04$ & $9.81 E-05$ & $3.22 \mathrm{E}-05$ & 2.6 \\
\hline S-R-296 & $02 \mathrm{WH}-14-\mathrm{A}$ & U234 & 4.84E-04 & $1.16 \mathrm{E}-04$ & $2.55 E-05$ & 4.2 \\
\hline S-R-296 & $02 \mathrm{WH}-14-\mathrm{A}$ & U238 & $3.98 E-04$ & $1.18 \mathrm{E}-04$ & $1.35 \mathrm{E}-05$ & 3.4 \\
\hline S-R-297 & $02 W H-15-A$ & U234 & 5.27E-04 & 1.26E-04 & 3.88E-05 & 4.2 \\
\hline S-R-297 & $02 W H-15-A$ & U238 & 3.96E-04 & 1.22E-04 & 4.24E-05 & 3.2 \\
\hline S-R-298 & $02 W H-16-A$ & U234 & $3.42 E-04$ & $1.01 E-04$ & $4.85 \mathrm{E}-05$ & 3.4 \\
\hline S-R-298 & $02 W H-16-A$ & U238 & $3.15 E-04$ & 1.07E-04 & $2.73 E-05$ & 2.9 \\
\hline S-R-299 & $02 \mathrm{WH}-17-\mathrm{A}$ & U234 & 3.39E-04 & 9.89E-05 & 3.46E-05 & 3.4 \\
\hline S-R-299 & 02WH-17-A & U238 & $3.39 E-04$ & $1.09 E-04$ & 3.07E-05 & 3.1 \\
\hline S-R-300 & $02 W H-18-A$ & U234 & 5.32E-04 & 1.16E-04 & 2.78E-05 & 4.6 \\
\hline S-R-300 & $02 W H-18-A$ & U238 & $3.99 \mathrm{E}-04$ & $1.12 \mathrm{E}-04$ & $2.78 \mathrm{E}-05$ & 3.6 \\
\hline S-R-301 & 02WH-19-A & U234 & $1.26 \mathrm{E}-02$ & $1.59 E-03$ & $3.76 \mathrm{E}-05$ & 7.9 \\
\hline S-R-301 & 02WH-19-A & U235 & $5.90 E-04$ & 1.35E-04 & 7.49E-05 & 4.4 \\
\hline S-R-301 & $02 W H-19-A$ & U238 & $1.34 \mathrm{E}-02$ & 1.92E-03 & 4.49E-05 & 7.0 \\
\hline S-R-302 & O2WH-20-A & PU238 & $6.47 E-03$ & 7.35E-04 & $6.90 \mathrm{E}-05$ & 8.8 \\
\hline S-R-302 & 02WH-20-A & PU239/240 & $5.20 E-03$ & 6.10E-04 & 8.17E-05 & 8.5 \\
\hline S-R-302 & $02 W H-20-A$ & U234 & $8.41 E-03$ & 9.65E-04 & 2.74E-05 & 8.7 \\
\hline S-R-302 & 02WH-20-A & U235 & 4.87E-04 & 9.45E-05 & 2.57E-05 & 5.2 \\
\hline S-R-302 & $02 W H-20-A$ & U238 & 8.14E-03 & $1.09 E-03$ & 2.74E-05 & 7.5 \\
\hline
\end{tabular}

All known sources of uncertainty are included in the uncertainty term. There may be unknown sources of uncertainty that are not accounted for. If the result/uncertainty ratio is more than 3 , we have a degree of confidence that the result is positive (i.e. the result is statistically different than zero). A result with the result/uncertainty ratio between 2 and 3 is the first indication that an isotope may be present and further investigation may be warranted.

As with any good science no single data point is used in important decisions (results need to be reproducible). 
RADIOANALYTICAL ANALYSES DATA PACKAGE

Project Title: $\quad$ Amchitka Island Alpha Analysis Report (Batch 14)

\begin{tabular}{|c|c|c|c|}
\hline Lab Name: & RTC & Case No: & NA \\
\hline Report No:: & AmchitBatchF14 & Method Type: & A/B \\
\hline Approved SAP No.: & NA & SDG No.: & K-AA-610 \\
\hline
\end{tabular}

\section{SAMPLE NUMBERS}

Customer

Sample ID

K-AA-610

K-AA-611

K-AA-612

K-AA-613

K-AA-614

K-AA-615

K-AA-616

K-AA-617

K-AA-618

K-AA-619

K-BB-600

K-BB-601

K-BB-602

K-BB-603

K-BB-604

K-BB-605

K-BB-606

K-BB-607

K-BB-608

K-BB-609
Lab Sample

ID

$02 \times 7-11-A$

$02 \times 7-12-A$

02X7-13-A

$02 \times 7-14-A$

$02 \times 7-15-A$

$02 \times 7-16-A$

$02 \times 7-17-A$

$02 \times 7-18-A$

$02 \times 7-19-A$

$02 \times 7-20-A$

$02 \times 7-01-A$

$02 \times 7-02-A$

$02 \times 7-03-A$

$02 \times 7-04-A$

$02 \times 7-05-A$

$02 \times 7-06-A$

$02 \times 7-07-A$

$02 \times 7-08-A$

$02 \times 7-09-A$

$02 \times 7-10-A$

Comments:

Release of the data contained in this data package has been authorized by the laboratory manager or the manager's designee, as verified by the following signature:

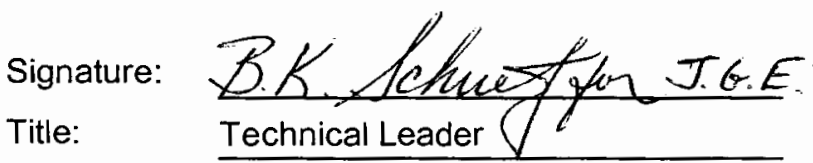

Name: J.G. Eisenmenger

Date: $\quad 11 / 22 / 2005$ 


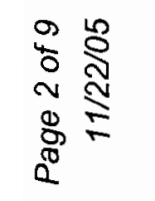

\begin{tabular}{|c|c|c|c|c|c|c|c|c|c|c|c|c|c|c|c|c|c|c|c|c|c|}
\hline $\begin{array}{l} \\
\\
\end{array}$ & & & & & & & & & & & & & & & & & & & & & \\
\hline$\stackrel{0}{2}$ & $\begin{array}{l}\text { n } \\
0 \\
1 \\
\text { ⿴囗玉 } \\
\sigma \\
\sigma \\
\stackrel{9}{9} \\
+\end{array}$ & $\begin{array}{l}n \\
0 \\
1 \\
1 \\
1 \\
0 \\
0 \\
0 \\
-1 \\
+ \\
+\end{array}$ & $\begin{array}{l}n \\
0 \\
1 \\
6 \\
2 \\
\sigma \\
\sigma \\
+ \\
+\end{array}$ & 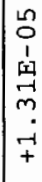 & 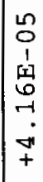 & 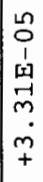 & $\begin{array}{l}n \\
0 \\
1 \\
1 \\
1 \\
\vdots \\
\sigma \\
\tilde{N} \\
+ \\
+\end{array}$ & 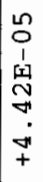 & 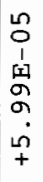 & $\begin{array}{l}n \\
0 \\
1 \\
\text { in } \\
0 \\
0 \\
\text { ñ } \\
+\end{array}$ & 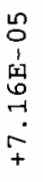 & $\begin{array}{l}0 \\
0 \\
1 \\
1 \\
0 \\
0 \\
-1 \\
o \\
+\end{array}$ & 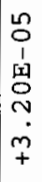 & 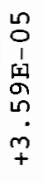 & 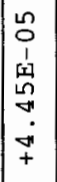 & \begin{tabular}{l}
2 \\
0 \\
1 \\
1 \\
\multicolumn{1}{c}{} \\
0 \\
0 \\
$\dot{0}$ \\
+
\end{tabular} & $\begin{array}{l}\text { n } \\
0 \\
1 \\
1 \\
1 \\
0 \\
0 \\
1 \\
+\end{array}$ & $\begin{array}{l}n \\
0 \\
1 \\
1 \\
0 \\
0 \\
\infty \\
m \\
+\end{array}$ & $\mid \begin{array}{l}n \\
0 \\
1 \\
1 \\
9 \\
0 \\
0 \\
m \\
+\end{array}$ & 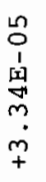 & $\begin{array}{c}n \\
0 \\
1 \\
\underline{n} \\
\tilde{N} \\
0 \\
\tilde{m} \\
+\end{array}$ \\
\hline 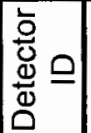 & ஜ & 18 & ¿ & $\begin{array}{l}{ }_{-} \\
-1\end{array}$ & 웅 & $\vec{F}$ & ${ }^{\circ}$ & 0 & $1-1$ & 10 & $\overrightarrow{-}$ & $\exists$ & ન્ & $\overrightarrow{0}$ & $\overrightarrow{0}$ & $\overrightarrow{0}$ & 5 & $\mp$ & 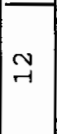 & $\underset{\rightarrow}{\stackrel{m}{*}}$ & 10 \\
\hline $\begin{array}{ll}\frac{E}{0} & \frac{\mathrm{g}}{0} \\
\frac{D}{\mathrm{O}} & \stackrel{0}{\nu}\end{array}$ & $\begin{array}{l}\sigma \\
\vdots \\
\infty \\
\sigma\end{array}$ & $\begin{array}{l}9 \\
\vdots \\
0\end{array}$ & $\begin{array}{l}\sigma \\
\infty \\
\sigma\end{array}$ & ने & ने & व. & $\dot{\sigma}$. & ร่ & g. & gं & $\stackrel{v}{m}$ & $\stackrel{\sim}{\sim}$ & $\begin{array}{l}m \\
\infty \\
o \\
o\end{array}$ & के & $\begin{array}{l}7 \\
\sigma \\
\sigma \\
\sigma\end{array}$ & $\begin{array}{l}-1 \\
\sigma \\
\sigma\end{array}$ & $\begin{array}{l}-1 \\
\sigma \\
\sigma\end{array}$ & $\begin{array}{l}\overrightarrow{1} \\
\dot{\infty} \\
\infty\end{array}$ & \begin{tabular}{|l|}
$\square$ \\
$\infty$ \\
$\infty$ \\
$\infty$
\end{tabular} & $\begin{array}{l}0 \\
\dot{8} \\
0 \\
0-1\end{array}$ & $\begin{array}{l}-1 \\
\sigma \\
\sigma\end{array}$ \\
\hline 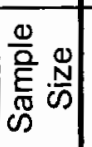 & $\stackrel{\sim}{\sim}$ & $\underset{\sim}{n}$ & $\stackrel{n}{\sim}$ & 吕 & 号 & 号 & $\bar{\sim}$ & In & 号 & $\stackrel{\Omega}{\curvearrowleft}$ & $\stackrel{n}{\sim}$ & ח & $\stackrel{n}{n}$ & 品 & $\begin{array}{ll}5 \\
-7\end{array}$ & \begin{tabular}{|l|}
\multirow{r}{*}{} \\
-1
\end{tabular} & $\begin{array}{l}5 \\
-10\end{array}$ & $\stackrel{\Omega}{\Omega}$ & 号 & 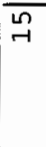 & $\stackrel{n}{\sim}$ \\
\hline
\end{tabular}

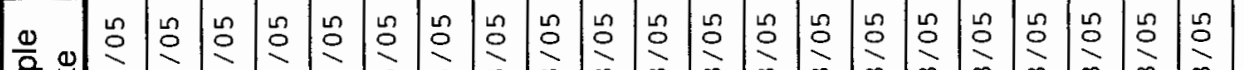

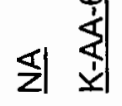

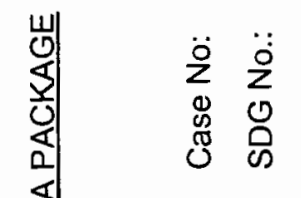

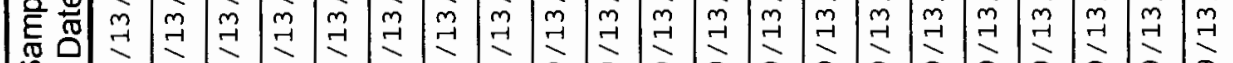

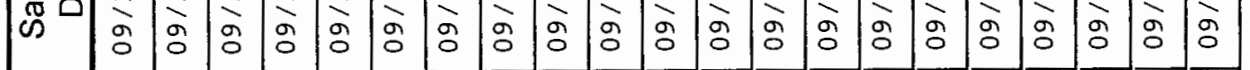

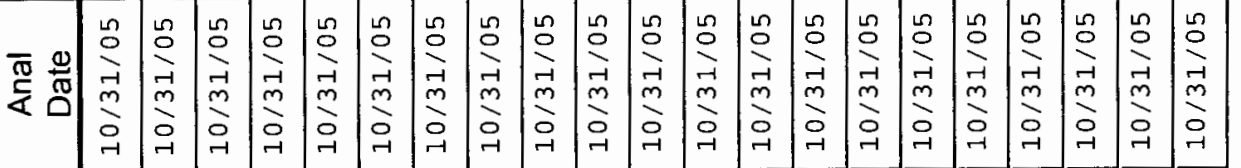

a a a a a a a a a

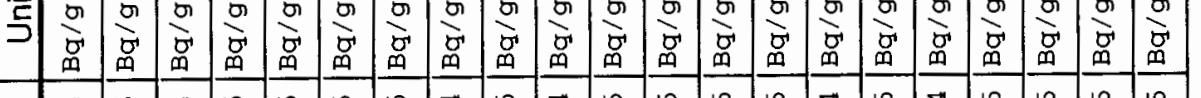

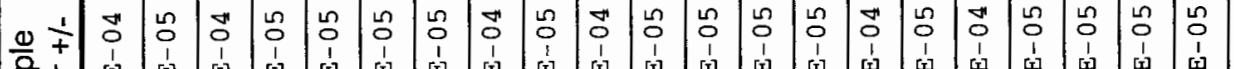

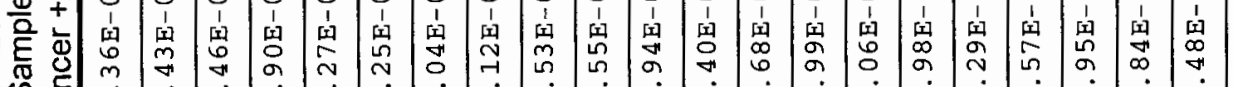

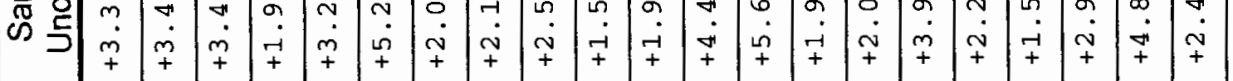
„2 है

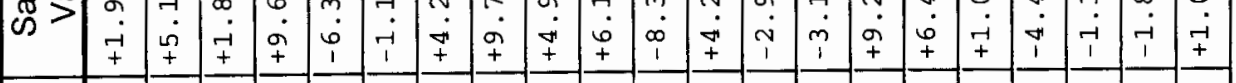

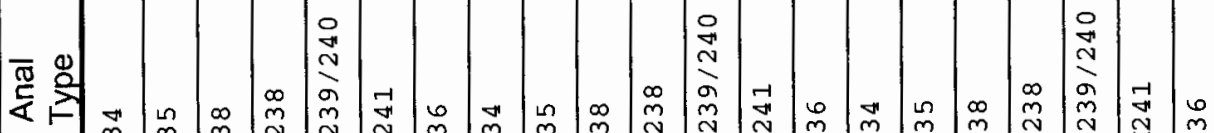
至

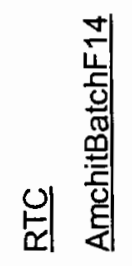

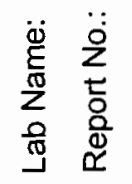

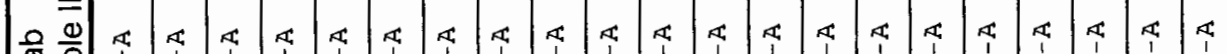

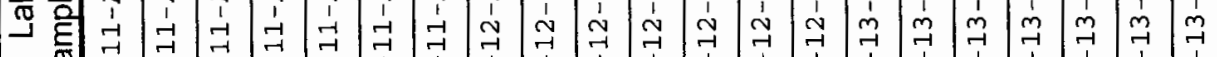

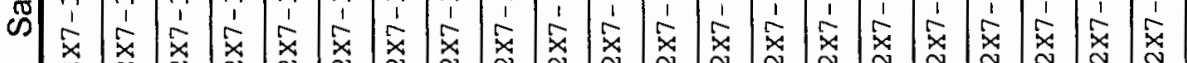

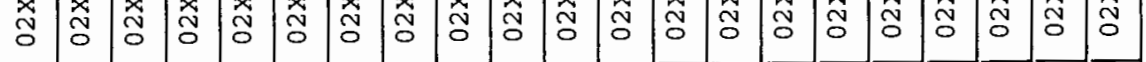

$\varrho$

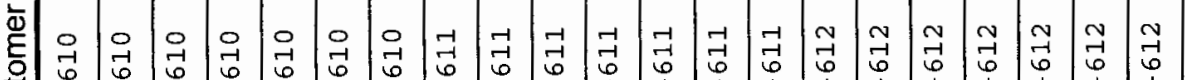

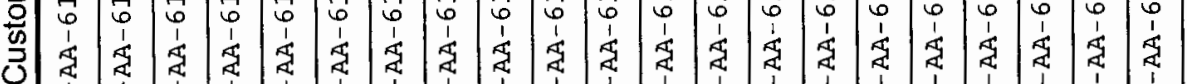

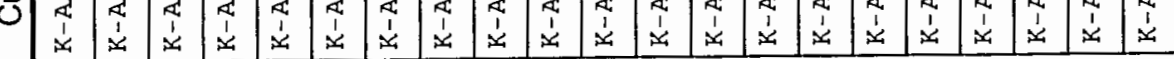




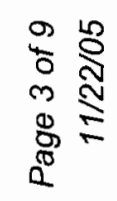

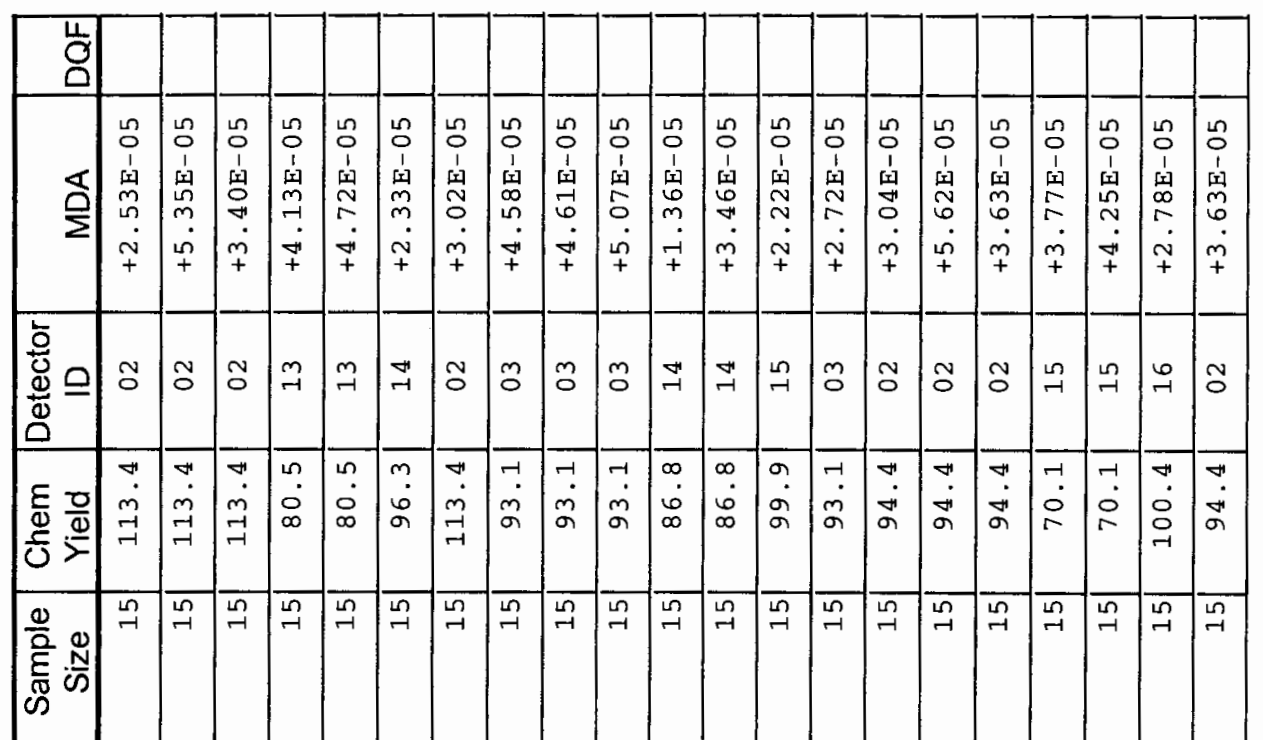

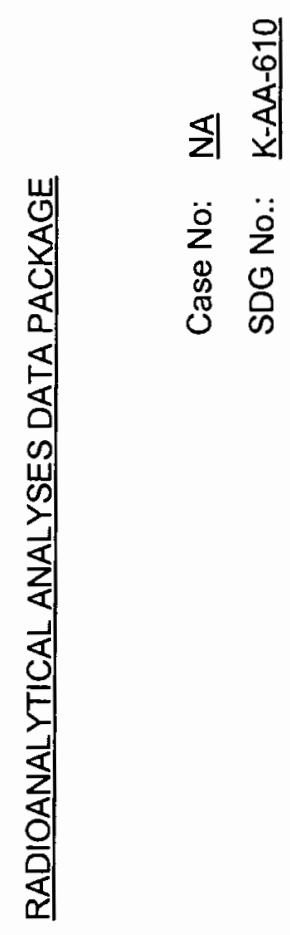

里 。

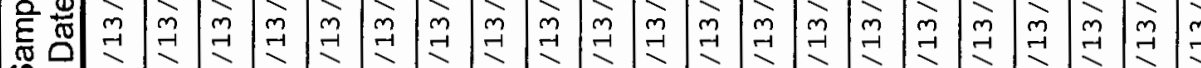

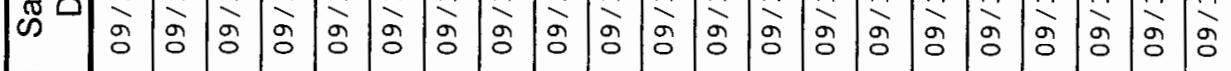

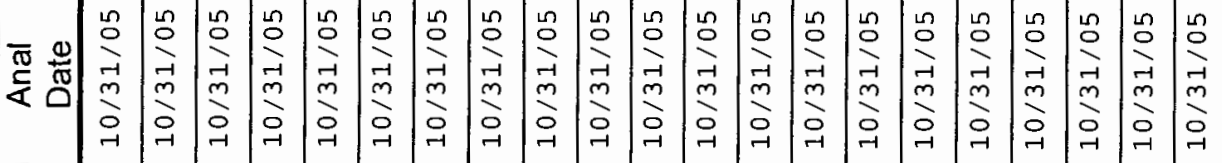

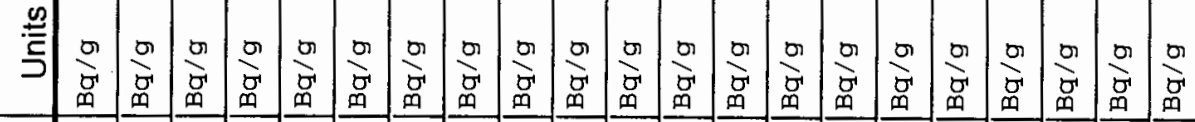
$\triangleq \frac{1}{f}$ 员

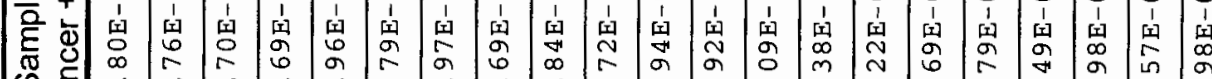

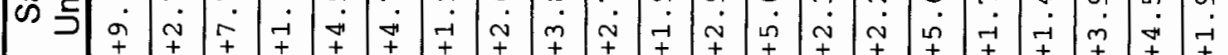
అ

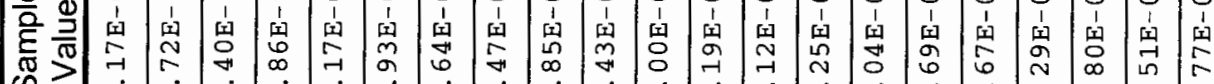

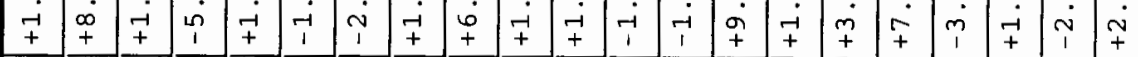
ত ఖ

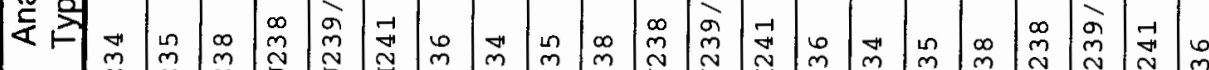

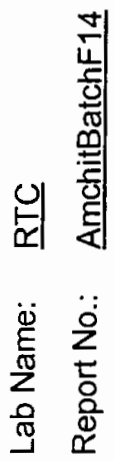

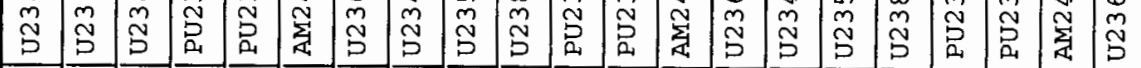
$\frac{\Phi}{\frac{0}{2}} \cdot \frac{x}{\bar{E}}$

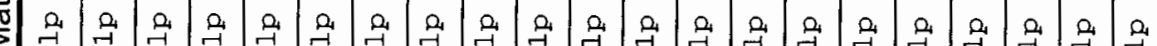

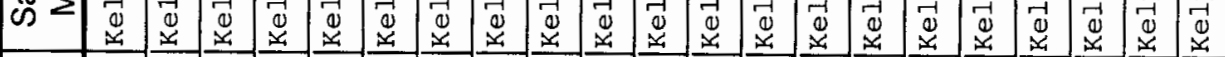

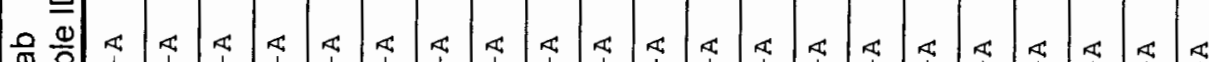

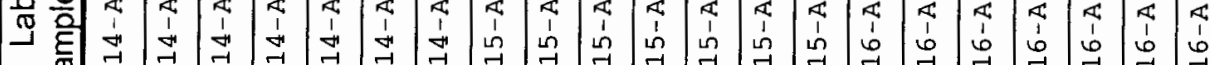
क

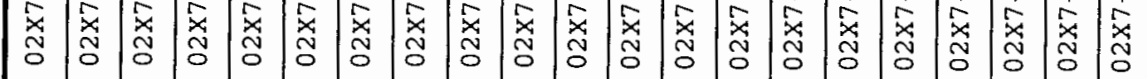

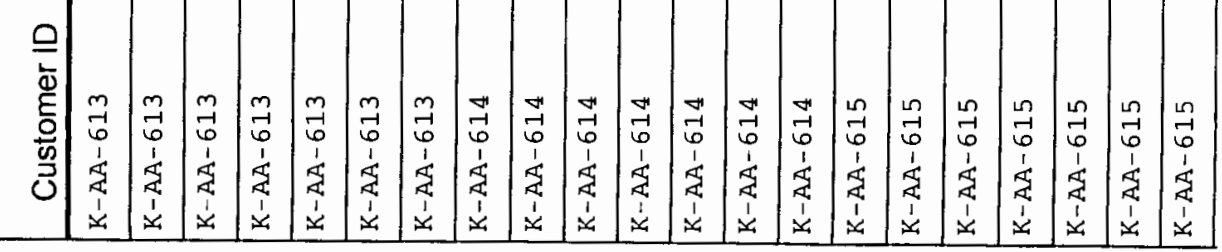




\begin{tabular}{|c|c|c|c|c|c|c|c|c|c|c|c|c|c|c|c|c|c|c|c|c|c|}
\hline $\begin{array}{l} \\
\\
0\end{array}$ & & & & & & & & & & & & & & & & & & & & & \\
\hline$\frac{4}{2}$ & $\begin{array}{l}n \\
0 \\
1 \\
1 \\
1 \\
\dot{1} \\
\infty \\
\dot{+1} \\
+\end{array}$ & $\begin{array}{l}n \\
0 \\
1 \\
1 \\
1 \\
0 \\
0 \\
0 \\
+ \\
+\end{array}$ & 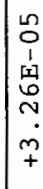 & $\begin{array}{l}\text { 음 } \\
1 \\
1 \\
1 \\
0 \\
0 \\
0 \\
0 \\
+\end{array}$ & $\begin{array}{c}2 \\
0 \\
1 \\
1 \\
0 \\
0 \\
0 \\
0 \\
+ \\
+\end{array}$ & 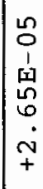 & $\begin{array}{l}n \\
0 \\
1 \\
1 \\
2 \\
6 \\
0 \\
0 \\
9 \\
+\end{array}$ & $\begin{array}{l}n \\
0 \\
1 \\
1 \\
01 \\
0 \\
0 \\
0 \\
0 \\
+\end{array}$ & $\begin{array}{l}20 \\
0 \\
1 \\
1 \\
\vdots \\
\vdots \\
0 \\
+ \\
+\end{array}$ & $\mid \begin{array}{l}n \\
0 \\
1 \\
1 \\
w \\
m \\
m \\
m \\
+ \\
+\end{array}$ & 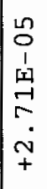 & $\begin{array}{l}n \\
0 \\
1 \\
1 \\
\\
\infty \\
0 \\
+1 \\
+ \\
+\end{array}$ & 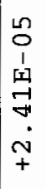 & 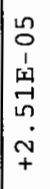 & $\begin{array}{c}\not \\
0 \\
1 \\
1 \\
1 \\
0 \\
0 \\
n \\
-1 \\
+\end{array}$ & 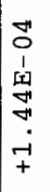 & 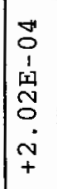 & 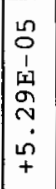 & $\begin{array}{l}\not \\
0 \\
1 \\
1 \\
0 \\
0 \\
0 \\
0 \\
-1 \\
+\end{array}$ & 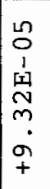 & $\begin{array}{l}0 \\
0 \\
1 \\
1 \\
1 \\
0 \\
0 \\
n \\
-1 \\
+\end{array}$ \\
\hline 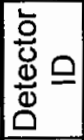 & ठే & $\overrightarrow{0}$ & I & $\mid$ & $\mid \begin{array}{c}0 \\
-1\end{array}$ & $\stackrel{m}{\rightarrow}$ & | & In & 迄 & $\stackrel{2}{\circ}$ & 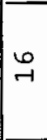 & 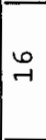 & $\vec{J}$ & 옹 & \% & 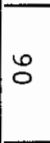 & 10 & $\stackrel{\circ}{\rightarrow}$ & 우 & $\stackrel{10}{\rightarrow-1}$ & $\stackrel{8}{0}$ \\
\hline$\frac{\frac{E}{\Phi}}{\frac{D}{0}}=$ & $\begin{array}{l}-1 \\
\infty \\
0 \\
0 \\
-1\end{array}$ & $\begin{array}{l}-1 \\
0 \\
0 \\
0 \\
-1\end{array}$ & $\begin{array}{l}-1 \\
\infty \\
0 \\
0 \\
-1\end{array}$ & ?. & ?. & $\begin{array}{l}\text { m. } \\
\text { d. } \\
\text { - } \\
\end{array}$ & $\begin{array}{l}-1 \\
-1 \\
\infty \\
0 \\
-1\end{array}$ & $\begin{array}{l}0 \\
\ddot{r} \\
r \\
-1\end{array}$ & $\begin{array}{l}0 \\
0 \\
-7 \\
7\end{array}$ & $\begin{array}{l}0 . \\
\hat{\sigma} \\
7\end{array}$ & $\begin{array}{l}\hat{n} \\
\infty \\
\infty\end{array}$ & 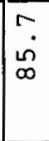 & $\begin{array}{l}\bar{\sigma} \\
\dot{n}\end{array}$ & $\begin{array}{l}0 \\
0 \\
0 \\
-1 \\
-1\end{array}$ & in & $\stackrel{\vec{\sim}}{\sim}$ & $\stackrel{\sim}{\sim}$ & $\overrightarrow{\tilde{N}}$ & $\overrightarrow{\tilde{N}}$ & $\dot{r}$ & $\stackrel{r}{\stackrel{n}{N}}$ \\
\hline 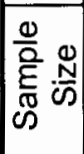 & $\stackrel{n}{\sim}$ & $\mid \begin{array}{l}\bar{n} \\
\rightarrow\end{array}$ & $\stackrel{\sim}{\sim}$ & $\stackrel{\sim}{\sim}$ & $\stackrel{\Omega}{\Omega}$ & $\stackrel{5}{\sim}$ & 吕 & in & 品 & 막 & In & 出 & $\stackrel{\Omega}{\sim}$ & $\stackrel{4}{\rightarrow}$ & 号 & ${ }_{\sim}^{n}$ & $\underset{r}{n}$ & $\bar{\Omega}$ & 吕 & $\underset{\sim}{\sim}$ & 늑 \\
\hline & & & & & & & & 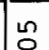 & & & D & 0 & 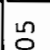 & 10 & ம & & n & 10 & n & مُ & 乞 \\
\hline
\end{tabular}

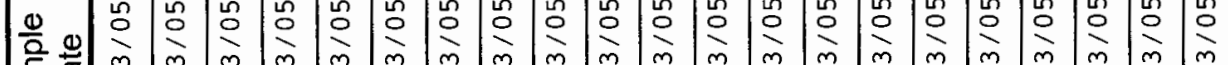

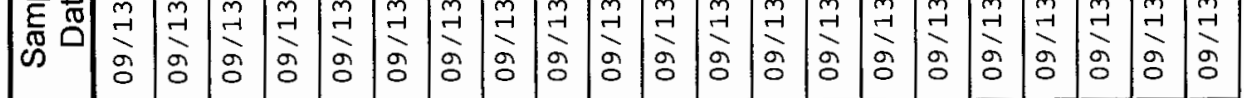

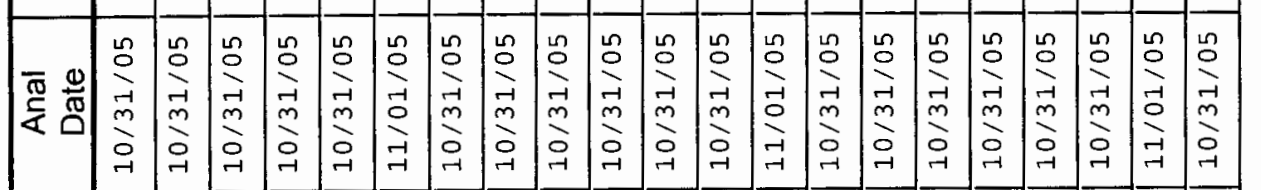

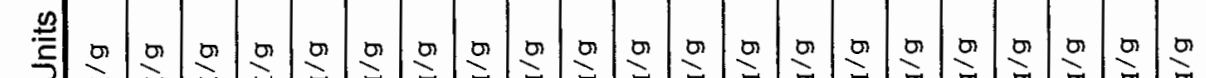

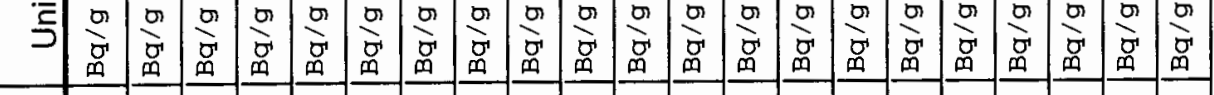

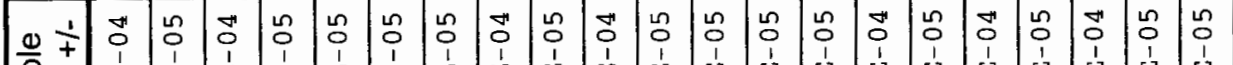

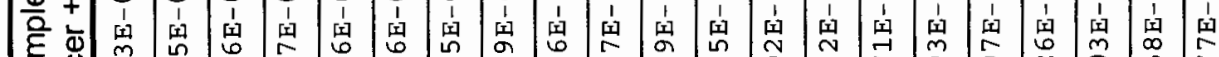
敢

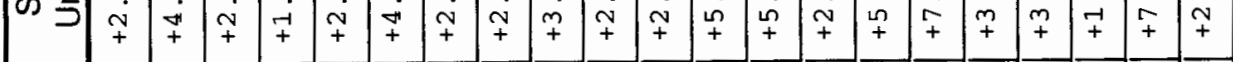
ब

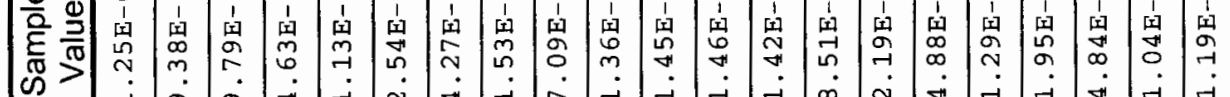

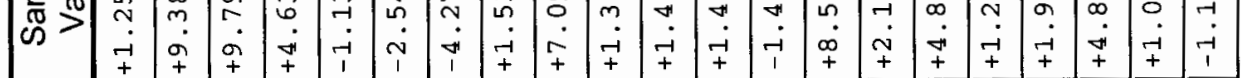


$\begin{array}{ll}0 \\ 8 \\ 0 \\ 0 \\ 0 & \frac{9}{2} \\ 0 & 2\end{array}$

\begin{tabular}{|c|c|c|c|c|c|c|c|c|c|c|c|c|c|c|c|c|c|c|c|c|c|}
\hline 늠 & & & & & & & & & & & & & & & & & & & & & \\
\hline$\stackrel{\partial}{\Sigma}$ & $\begin{array}{l}n \\
0 \\
1 \\
9 \\
9 \\
\vec{y} \\
\stackrel{5}{+} \\
+\end{array}$ & $\begin{array}{l}n \\
0 \\
1 \\
\text { yn } \\
1 \\
m \\
\dot{0} \\
+ \\
+\end{array}$ & 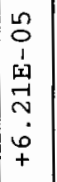 & 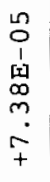 & $\begin{array}{l}0 \\
0 \\
1 \\
0 \\
0 \\
0 \\
0 \\
0 \\
+\end{array}$ & 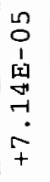 & 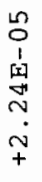 & 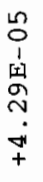 & 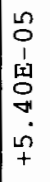 & $\begin{array}{l}n \\
0 \\
1 \\
1 \\
1 \\
6 \\
0 \\
9 \\
+ \\
+\end{array}$ & $\begin{array}{c}0 \\
0 \\
1 \\
1 \\
9 \\
0 \\
0 \\
-7 \\
+\end{array}$ & $\begin{array}{l}5 \\
0 \\
1 \\
1 \\
7 \\
n \\
-1 \\
+ \\
+\end{array}$ & 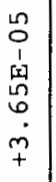 & 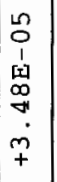 & 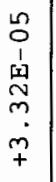 & 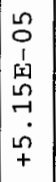 & 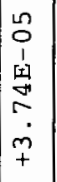 & 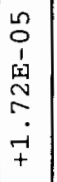 & $\mid \begin{array}{l}n \\
0 \\
1 \\
1 \\
0 \\
0 \\
\vdots \\
0 \\
+ \\
+\end{array}$ & 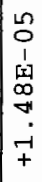 & $\mid \begin{array}{l}n \\
0 \\
1 \\
1 \\
01 \\
0 \\
0 \\
0 \\
0 \\
+\end{array}$ \\
\hline 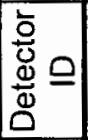 & $\stackrel{\infty}{\circ}$ & ${ }_{0}^{\infty}$ & $\stackrel{\infty}{\circ}$ & 10 & ó & $\overrightarrow{0}$ & $\stackrel{\infty}{\circ}$ & ô & 5 & ${ }_{0}^{-1}$ & of & I & g & 10 & $\tilde{O}$ & 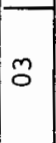 & $\stackrel{m}{o}$ & $\stackrel{ㅇ}{\rightarrow}$ & 10 & $\mid \begin{array}{r}0 \\
-1\end{array}$ & $\stackrel{m}{o}$ \\
\hline 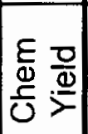 & $\begin{array}{l}? \\
-1 \\
0 \\
-1\end{array}$ & $\begin{array}{l}0 \\
n \\
-1 \\
0 \\
n-1\end{array}$ & \begin{tabular}{|c|}
$n$ \\
- \\
0 \\
0 \\
-1 \\
\end{tabular} & $\begin{array}{l} \\
\dot{m} \\
\dot{0}\end{array}$ & के & 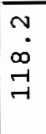 & $\begin{array}{l}\text { n? } \\
\text { ? }\end{array}$ & $\begin{array}{l}\infty \\
\dot{N} \\
\dot{N}\end{array}$ & $\begin{array}{l}\infty \\
\dot{\infty} \\
\dot{0} \\
-1\end{array}$ & $\begin{array}{l} \\
\dot{N} \\
0 \\
\sim \\
\sim\end{array}$ & \begin{tabular}{|l|}
$m$ \\
$a$ \\
$\infty$ \\
$v$ \\
\end{tabular} & \begin{tabular}{|l|}
$m$ \\
$\infty$ \\
$N$ \\
$N$
\end{tabular} & $\begin{array}{l}\sigma \\
\dot{j} \\
\sigma\end{array}$ & $\begin{array}{l}\infty \\
\dot{0} \\
0 \\
0 \\
-\end{array}$ & $\begin{array}{l}\vec{r} \\
\dot{a} \\
\sigma\end{array}$ & $\begin{array}{l} \\
\dot{A} \\
\sigma\end{array}$ & $\begin{array}{l}\vec{y} \\
\ddot{-} \\
\sigma\end{array}$ & $\begin{array}{l} \\
\\
0 \\
\end{array}$ & $\begin{array}{l}0 \\
\dot{0} \\
\end{array}$ & $\begin{array}{l}\sigma \\
\sigma \\
-1 \\
-1\end{array}$ & $\begin{array}{l}\vec{y} \\
\dot{\gamma}\end{array}$ \\
\hline
\end{tabular}

递

§| 题|

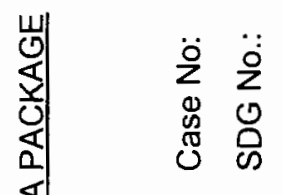

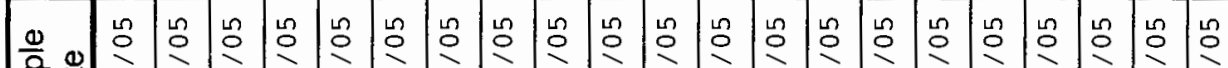

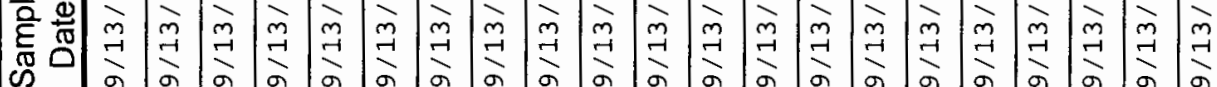

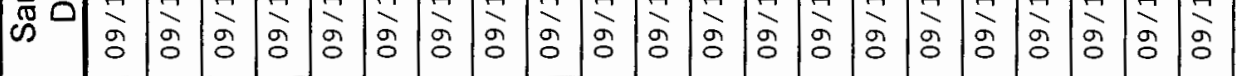

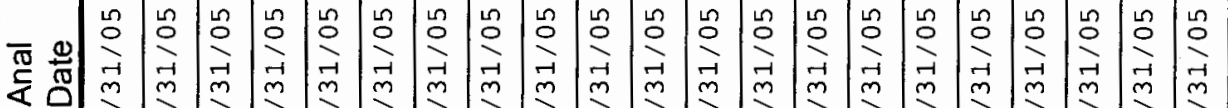

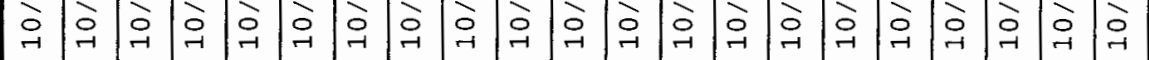

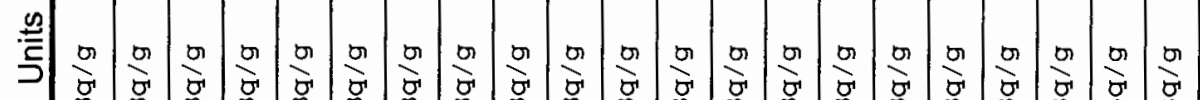

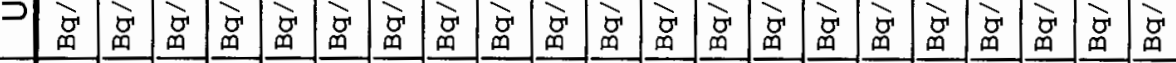

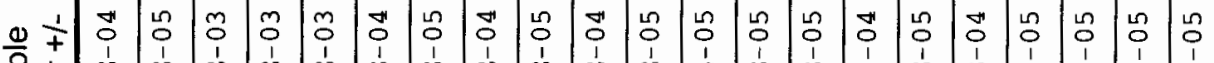

焉

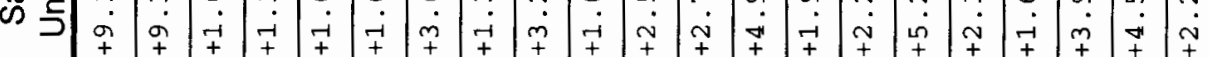

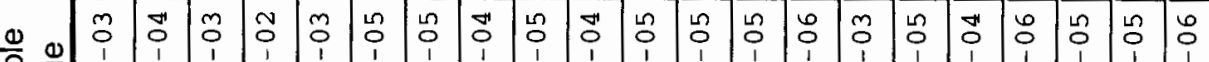

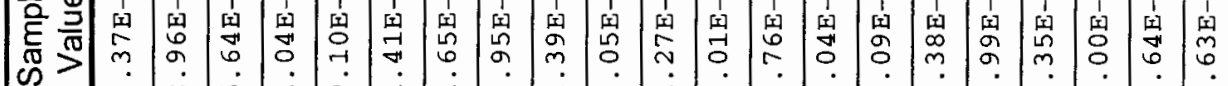

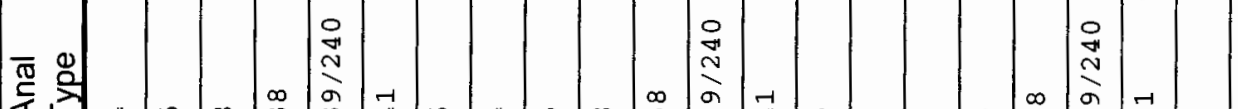

임

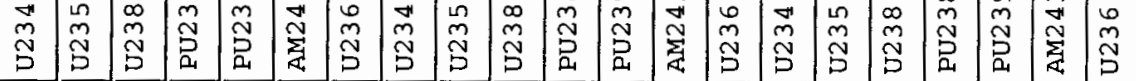

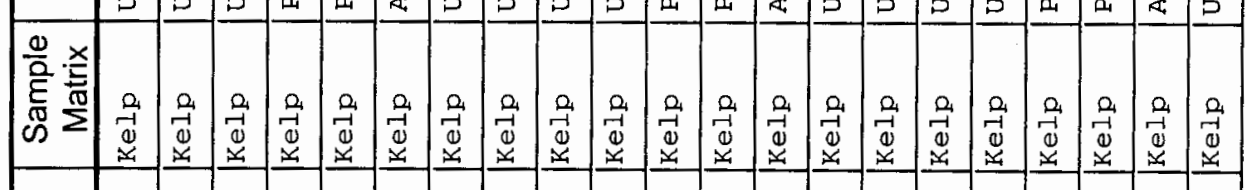

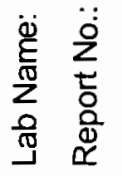

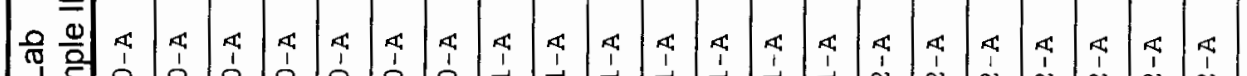

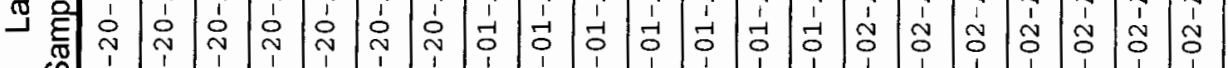

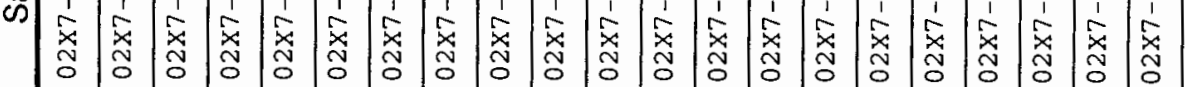

అ

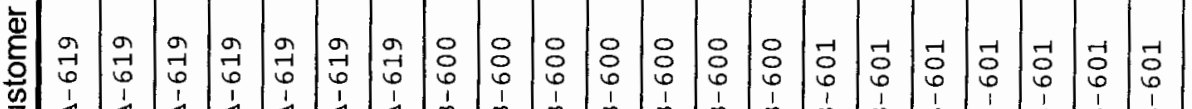

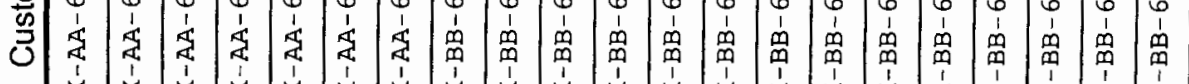




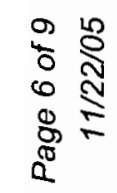

\begin{tabular}{|c|c|c|c|c|c|c|c|c|c|c|c|c|c|c|c|c|c|c|c|c|c|}
\hline$\stackrel{\nwarrow}{\mathrm{d}}$ & 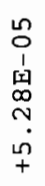 & $\begin{array}{l}0 \\
0 \\
1 \\
1 \\
0 \\
\infty \\
\infty \\
+ \\
+\end{array}$ & $\begin{array}{l}n \\
0 \\
1 \\
\text { cr } \\
\infty \\
+ \\
+ \\
+ \\
+\end{array}$ & 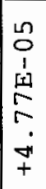 & 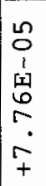 & 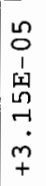 & $\begin{array}{l}\text { L } \\
0 \\
1 \\
1 \\
\mathfrak{1} \\
\infty \\
\infty \\
\dot{m} \\
\dot{m} \\
+\end{array}$ & $\begin{array}{l}\text { L } \\
\text { 1 } \\
1 \\
\text { 19 } \\
m \\
m \\
m \\
+ \\
+\end{array}$ & 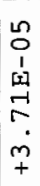 & 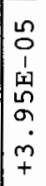 & 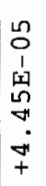 & 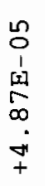 & 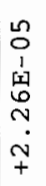 & 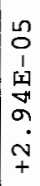 & 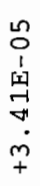 & 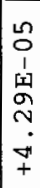 & $\begin{array}{l}\text { ㅇ } \\
0 \\
1 \\
\text { c1 } \\
0 \\
\infty \\
\vdots \\
\dot{+} \\
+\end{array}$ & $\begin{array}{l}\text { Ln } \\
0 \\
1 \\
1 \\
m \\
m \\
\stackrel{1}{+} \\
\stackrel{0}{+} \\
+\end{array}$ & 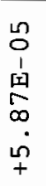 & $\begin{array}{l}10 \\
0 \\
1 \\
\text { [t] } \\
\sigma \\
\sigma \\
\sigma \\
\dot{N} \\
+\end{array}$ & 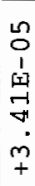 \\
\hline 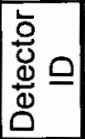 & $~_{0}^{\prime \prime}$ & ઠ゙ & Ö & $\exists$ & $\Rightarrow$ & $\stackrel{-}{-}$ & ○゙ & In & Ln & In & $\underset{r}{N}$ & $\underset{-}{\mathfrak{r}}$ & $\stackrel{\overbrace{}}{\sim}$ & n & ○̊ & $\mathscr{0}$ & \% & $\stackrel{m}{\rightarrow}$ & $\stackrel{m}{\rightarrow}$ & $\stackrel{m}{\rightarrow}$ & 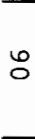 \\
\hline 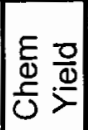 & $\begin{array}{l}\infty \\
\dot{m} \\
\stackrel{-}{-}\end{array}$ & $\begin{array}{l}\infty \\
\dot{m} \\
\dot{m} \\
\sim\end{array}$ & $\begin{array}{l}\infty \\
\dot{m} \\
0 \\
\dot{\gamma}\end{array}$ & in & in & $\begin{array}{l}\infty \\
\infty \\
\sigma\end{array}$ & $\begin{array}{l}\infty \\
\dot{0} \\
\dot{n} \\
0 \\
r-1\end{array}$ & ने & م. & बं & $\begin{array}{l}\because \\
0 \\
0\end{array}$ & $\dot{0}$ & $\begin{array}{r}0 \\
0 \\
0 \\
r\end{array}$ & r. & $\begin{array}{l}\infty \\
\dot{v} \\
\dot{\sigma} \\
-\end{array}$ & $\begin{array}{l}\infty \\
\dot{0} \\
\stackrel{0}{-}\end{array}$ & $\begin{array}{l}\infty \\
\dot{0} \\
\dot{0} \\
-1\end{array}$ & $\begin{array}{l}\infty \\
\overrightarrow{0}\end{array}$ & 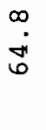 & $\begin{array}{l}-1 \\
\dot{0} \\
\stackrel{0}{r}\end{array}$ & 文 \\
\hline 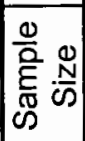 & L & $\prod_{n-1}^{n}$ & In & $\stackrel{n}{n}$ & n & 吕 & 吕 & م & ח & 足 & (5) & ñ & ח & 5 & $\stackrel{\sim}{\sim}$ & $\prod_{-1}$ & 省 & 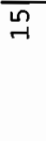 & n & 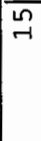 & $\prod_{r \rightarrow-1}^{\infty}$ \\
\hline
\end{tabular}

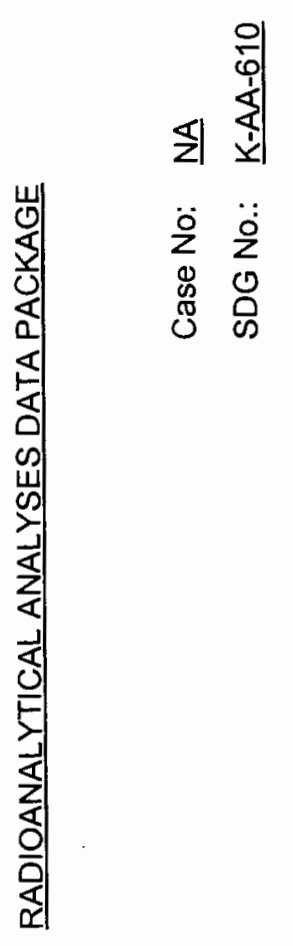

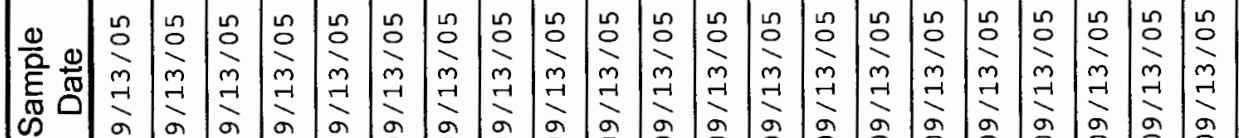
ऊ ळ

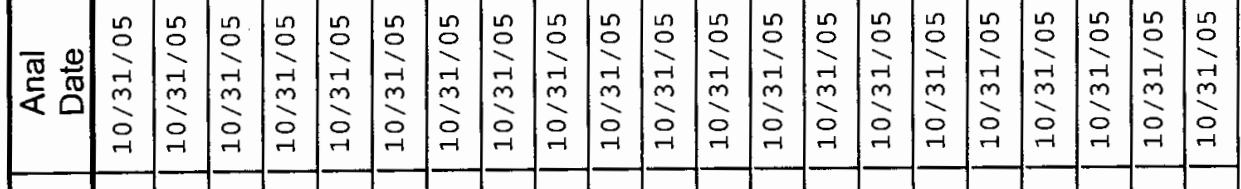

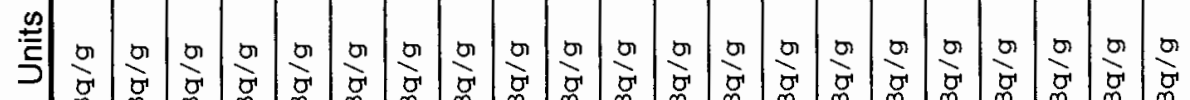

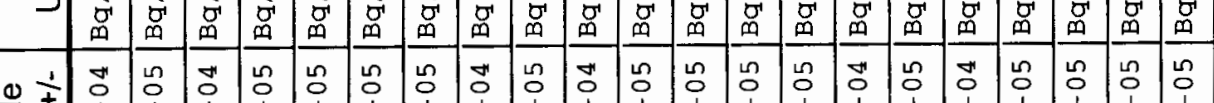

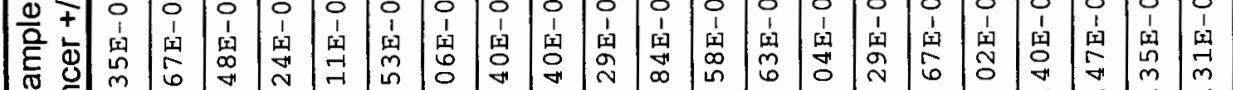

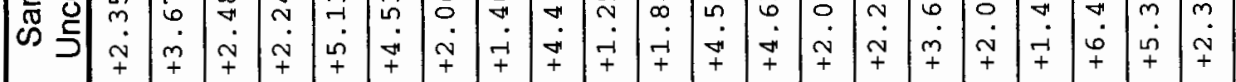

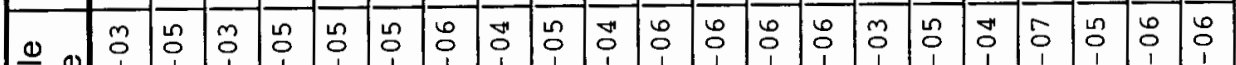

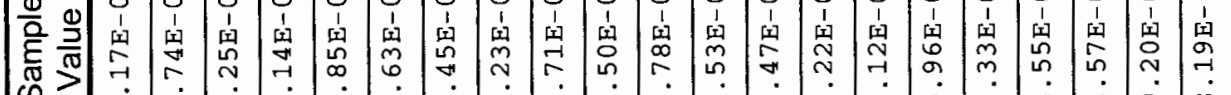

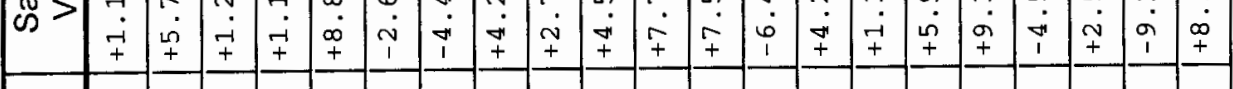

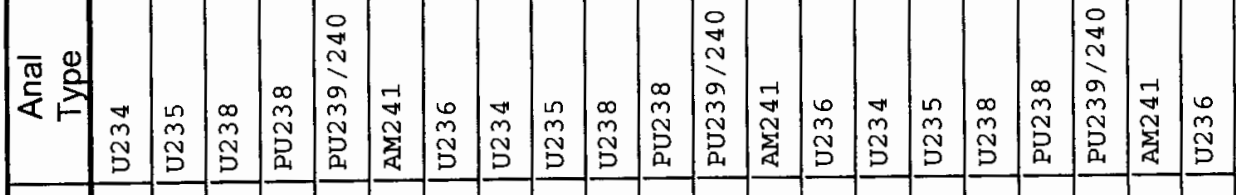

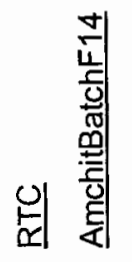

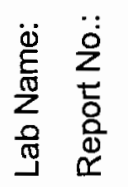

\begin{tabular}{|c|c|c|c|c|c|c|c|c|c|c|c|c|c|c|c|c|c|c|c|c|}
\hline 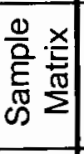 & \begin{tabular}{|l}
0 \\
0 \\
0 \\
$x$ \\
$x$
\end{tabular} & 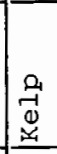 & 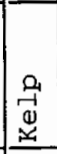 & 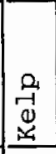 & 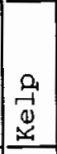 & \begin{tabular}{|l|}
$\stackrel{2}{a}$ \\
$\overrightarrow{0}$ \\
$\ddot{\sim}$
\end{tabular} & 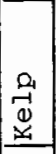 & 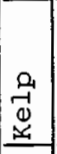 & 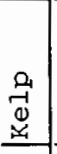 & 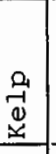 & 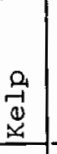 & $\begin{array}{l}\Omega_{1} \\
\mathbb{\Phi} \\
\underline{\simeq} \\
\end{array}$ & 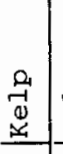 & $\begin{array}{l}0 \\
0 \\
0 \\
\underline{a} \\
\end{array}$ & 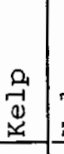 & 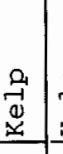 & 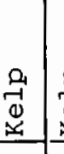 & 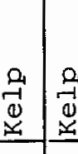 & 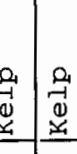 & 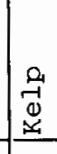 \\
\hline 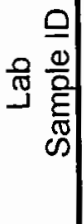 & 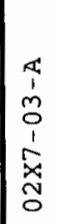 & 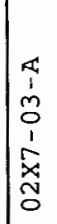 & 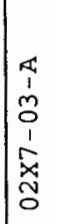 & 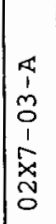 & 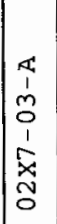 & $\mid \begin{array}{l}1 \\
1 \\
0 \\
0 \\
1 \\
1 \\
x \\
x \\
0 \\
0\end{array}$ & 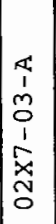 & 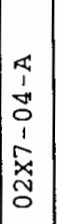 & $\begin{array}{l}\mathbb{4} \\
1 \\
0 \\
0 \\
1 \\
x \\
x \\
0 \\
0\end{array}$ & $\begin{array}{l}\mathbb{1} \\
1 \\
\forall \\
0 \\
1 \\
\tilde{x} \\
\tilde{z} \\
0\end{array}$ & 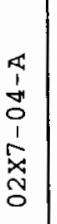 & 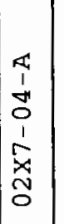 & 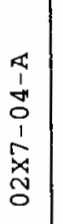 & 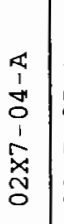 & $\begin{array}{l}\alpha \\
1 \\
1 \\
0 \\
1 \\
1 \\
x \\
x \\
0 \\
0\end{array}$ & 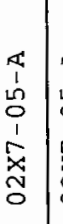 & 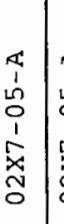 & 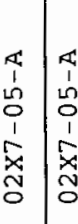 & 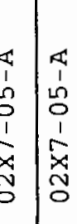 & $\begin{array}{l}\tilde{a} \\
1 \\
1 \\
0 \\
0 \\
1 \\
\hat{x} \\
\tilde{x} \\
0 \\
0\end{array}$ \\
\hline 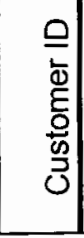 & $\begin{array}{l}0 \\
0 \\
0 \\
1 \\
m \\
0 \\
1 \\
1 \\
x\end{array}$ & 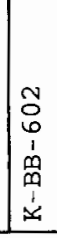 & 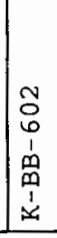 & 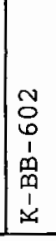 & \begin{tabular}{|l|} 
\\
0 \\
0 \\
0 \\
1 \\
0 \\
0 \\
1 \\
1 \\
\\
\end{tabular} & \begin{tabular}{|l|} 
\\
0 \\
0 \\
0 \\
1 \\
0 \\
0 \\
1 \\
\\
\end{tabular} & 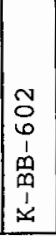 & 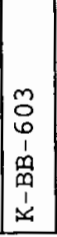 & $\begin{array}{l}m \\
0 \\
0 \\
1 \\
m \\
\infty \\
1 \\
1 \\
1 \\
\end{array}$ & \begin{tabular}{l}
$m$ \\
0 \\
0 \\
1 \\
$m$ \\
0 \\
1 \\
1 \\
\hdashline
\end{tabular} & $\begin{array}{l}m \\
0 \\
0 \\
1 \\
1 \\
0 \\
1 \\
1 \\
x \\
\end{array}$ & $\begin{array}{c}m \\
0 \\
0 \\
1 \\
0 \\
0 \\
1 \\
1 \\
2 \\
\end{array}$ & 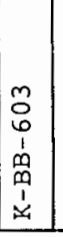 & $\begin{array}{l}m \\
0 \\
0 \\
1 \\
m \\
m \\
1 \\
1 \\
1\end{array}$ & 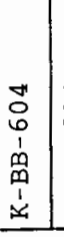 & $\begin{array}{l}07 \\
0 \\
0 \\
1 \\
0 \\
0 \\
1 \\
1 \\
\\
\end{array}$ & 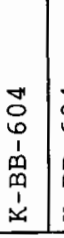 & 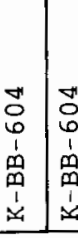 & 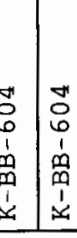 & 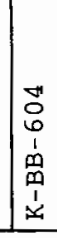 \\
\hline
\end{tabular}




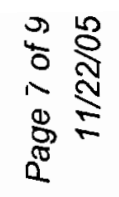

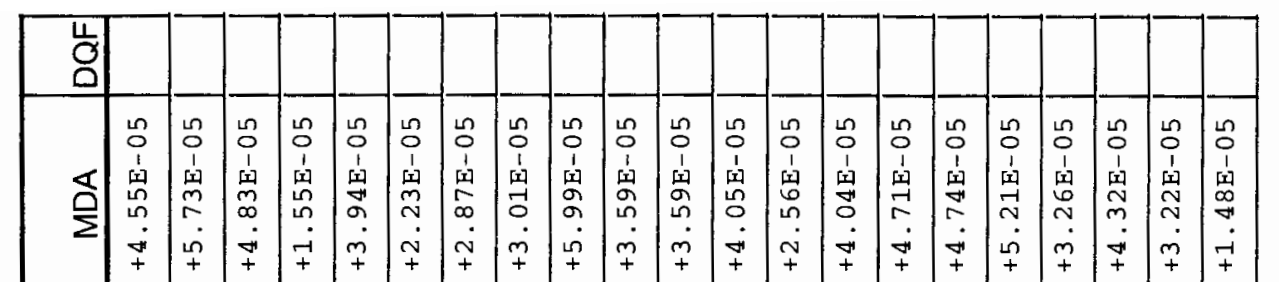

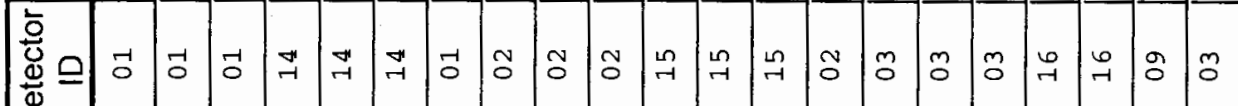
Ф

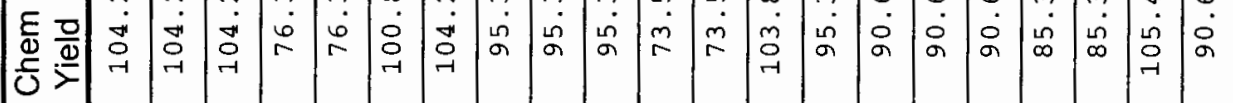

我新

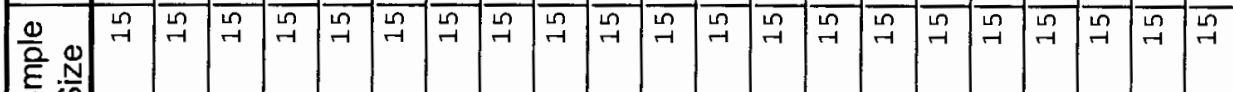

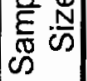

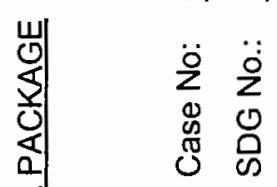

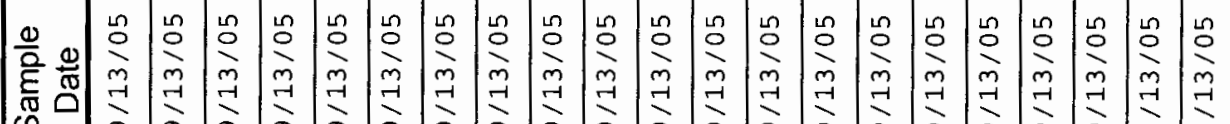

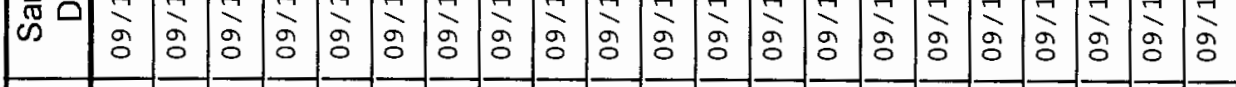

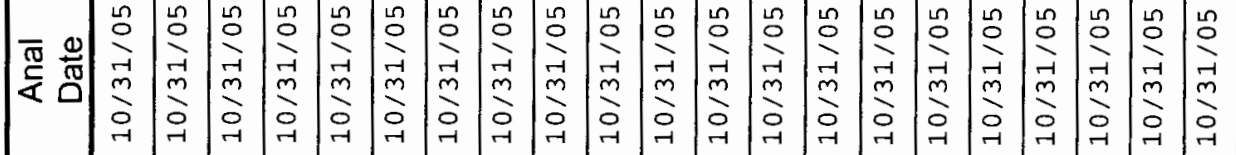

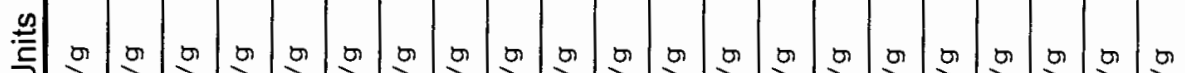

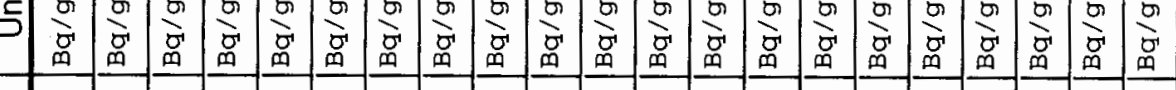

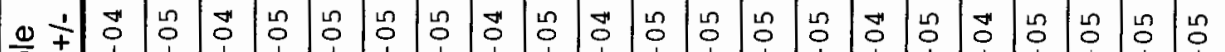

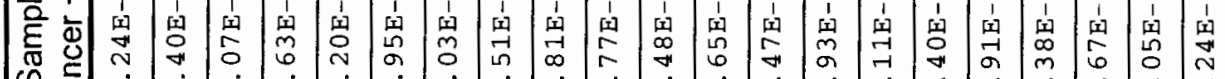
is

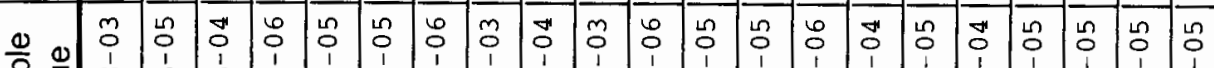

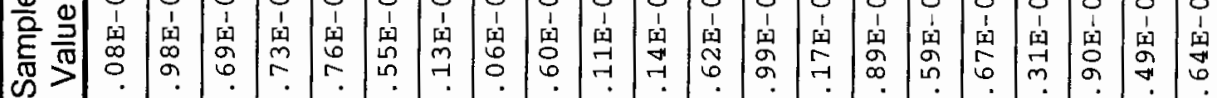

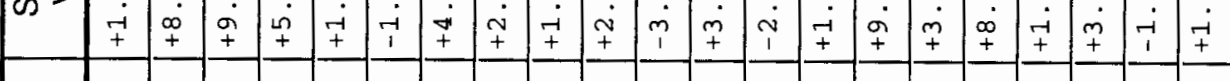

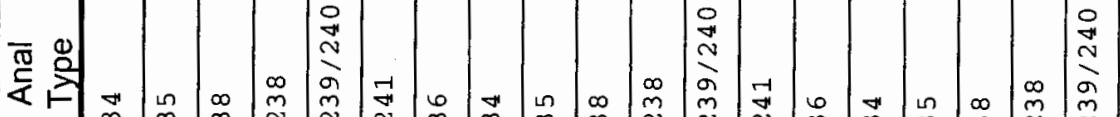

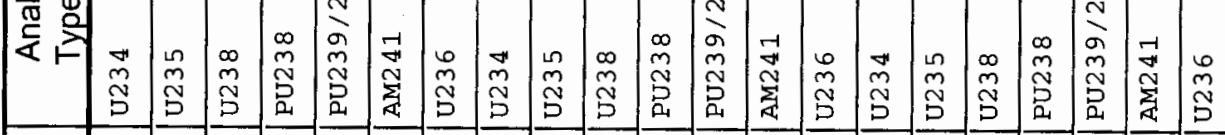

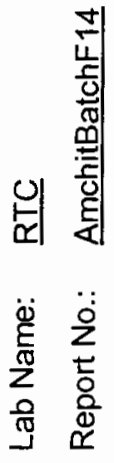

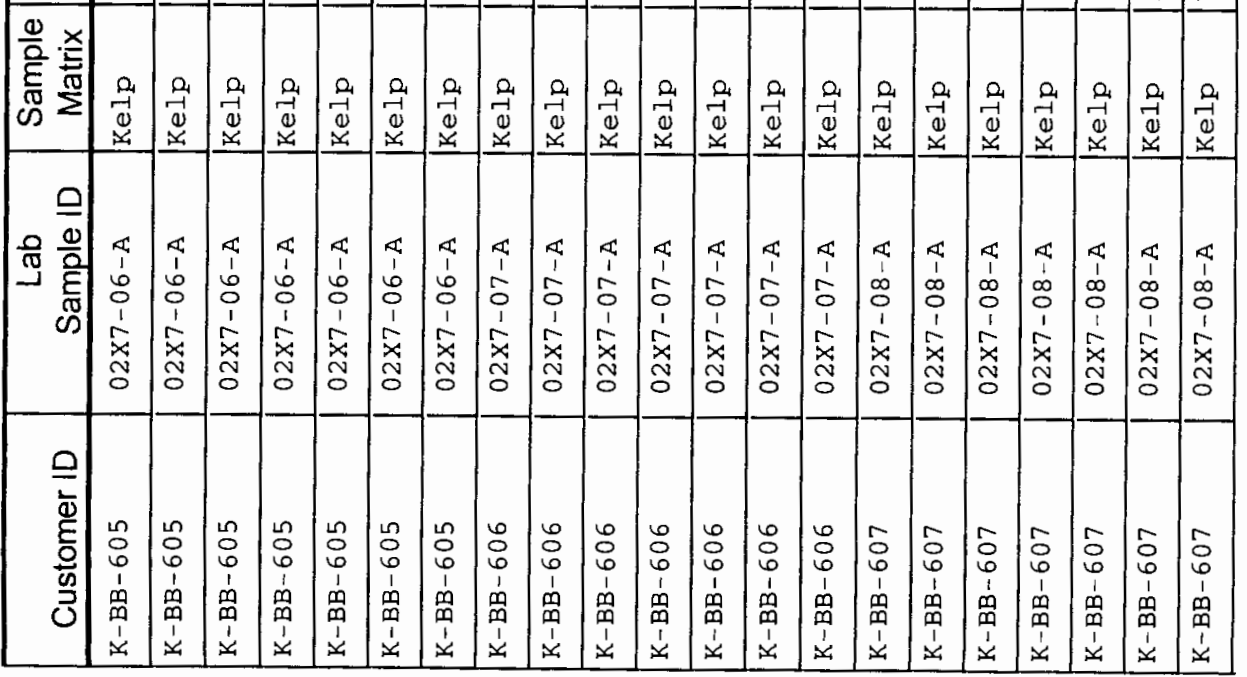




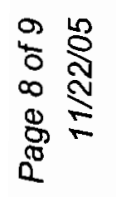

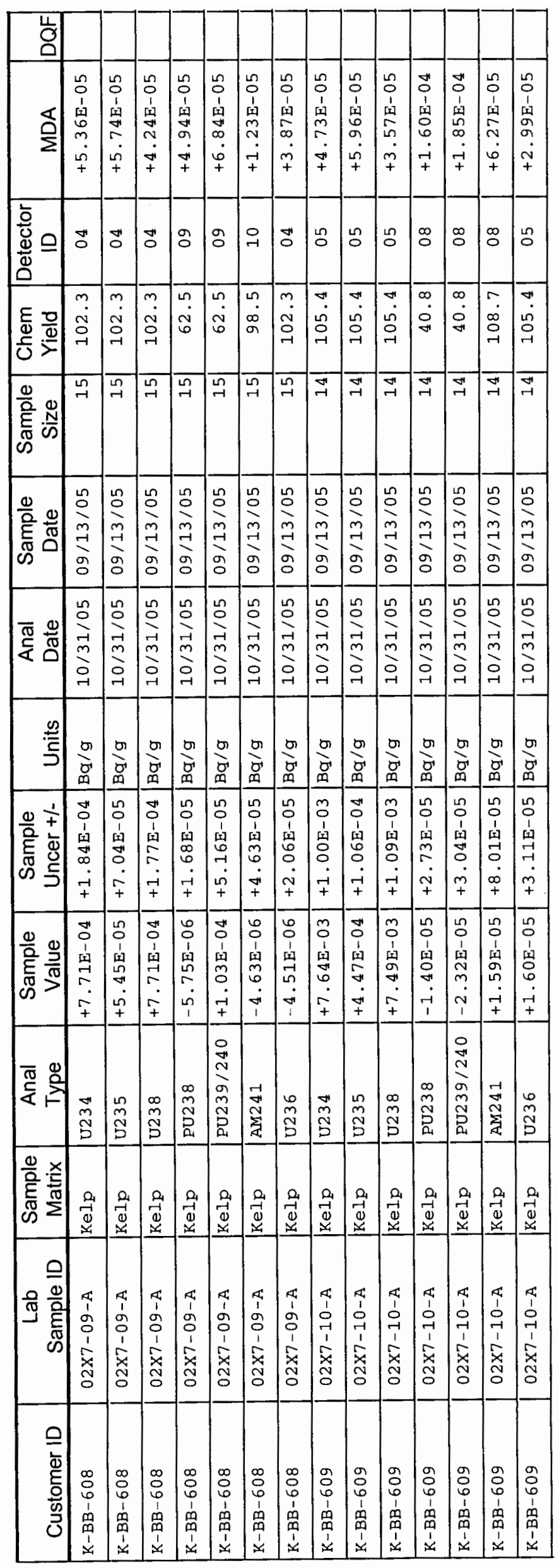

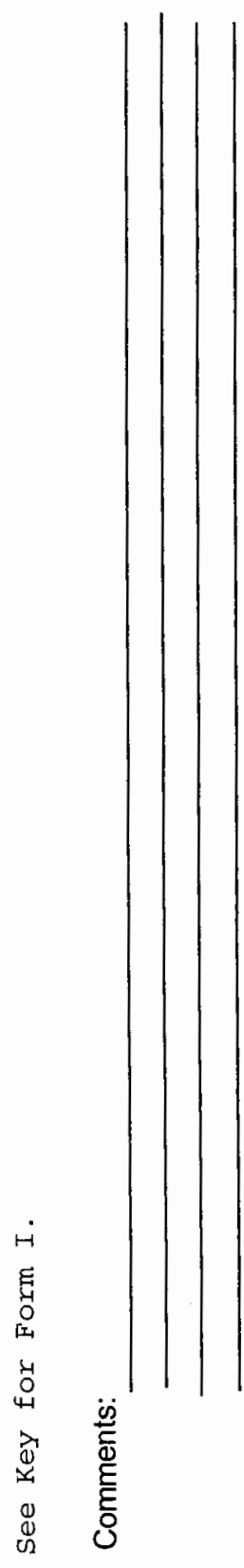




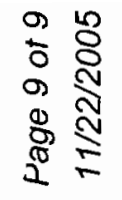

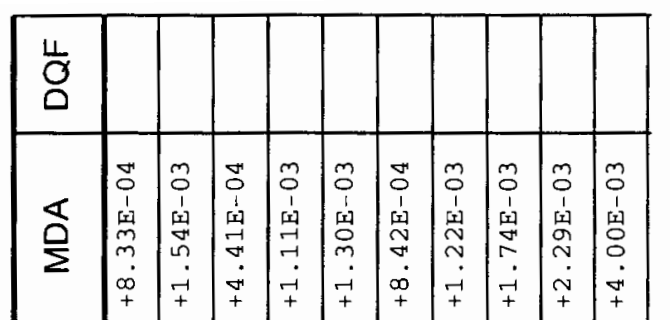

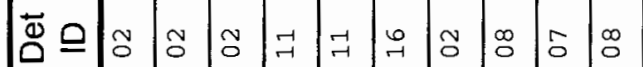

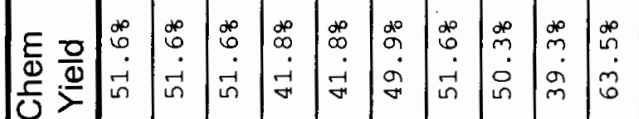

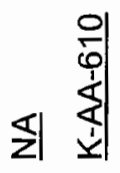

蓄高总

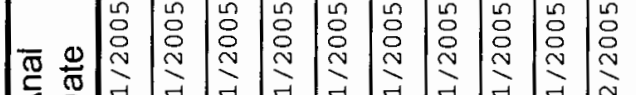

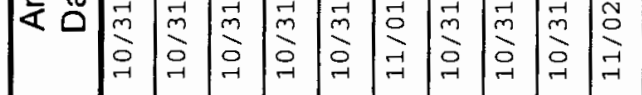

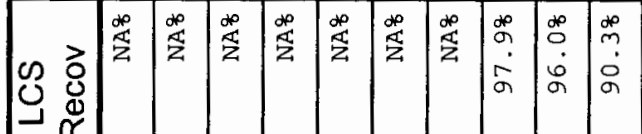

竞

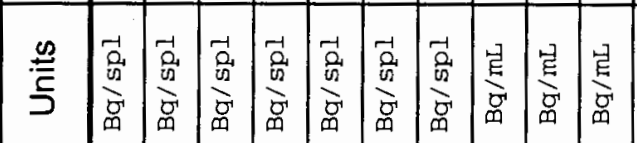

势竞 产

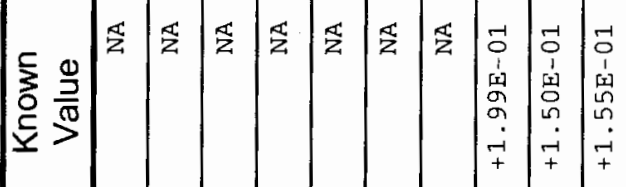

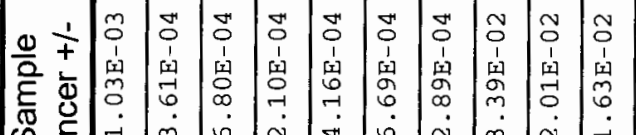

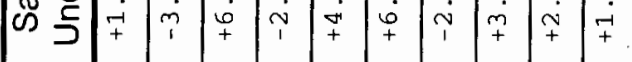

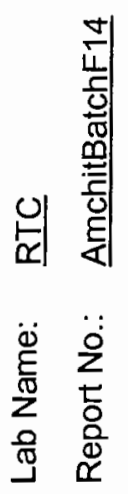

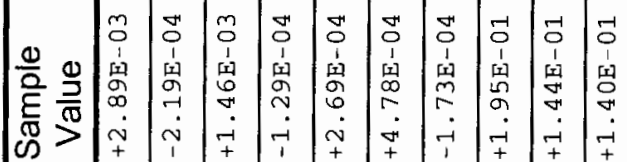

\begin{tabular}{|c|c|c|c|c|c|c|c|c|c|c|}
\hline$\frac{\stackrel{0}{\stackrel{0}{1}}}{\frac{\pi}{\frac{\pi}{2}}}$ & 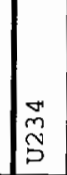 & 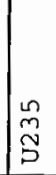 & 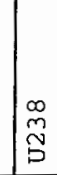 & 交 & 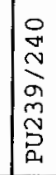 & 㼛 & $\mid \begin{array}{l}0 \\
m \\
\stackrel{\rho}{b}\end{array}$ & $\begin{array}{l}\infty \\
\stackrel{\infty}{D} \\
D\end{array}$ & 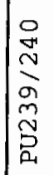 & 专 \\
\hline 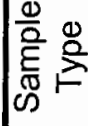 & 盖 & 善 & 窇 & 莴 & 学 & 岑 & 皆 & 号 & $\underset{త ్}{0}$ & ्ְత్ \\
\hline 囟 & 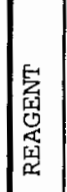 & 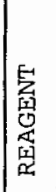 & 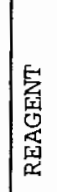 & 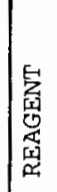 & 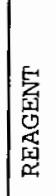 & 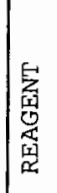 & 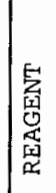 & 岛 & 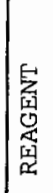 & 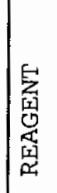 \\
\hline
\end{tabular}


. 
Project: $\quad$ Alpha Analysis for Amchitka Island (Batch 14)

Laboratory: RTC

Report \#: AmchitBatchF14

SDG\#: $\quad$ K-AA-610

\section{Summary of 2 and 3 sigma activities}

Below are the results for U234, U235, U236, U238, Pu238, Pu239/240, and Am241 for Batch 13 from the Amchitka Island Project that had a result/uncertainty ratio of 2 or more (uncertainty @ one sigma).

\begin{tabular}{|c|c|c|c|c|c|c|}
\hline $\begin{array}{l}\text { Customer } \\
\text { ID }\end{array}$ & Lab ID & Isotope & $\begin{array}{l}\text { Result } \\
\text { Bq/g }\end{array}$ & $\begin{array}{l}\text { Uncertainty } \\
\mathrm{Bq} / \mathrm{g}\end{array}$ & MDA & $\begin{array}{c}\text { Result/ } \\
\text { Uncertainty }\end{array}$ \\
\hline K-AA-610 & $02 \times 7-11-A$ & U234 & $1.95 \mathrm{E}-03$ & 3.36E-04 & $3.99 \mathrm{E}-05$ & 5.8 \\
\hline K-AA-610 & $02 \times 7-11-A$ & U238 & 1.89E-03 & $3.46 \mathrm{E}-04$ & 4.99E-05 & 5.5 \\
\hline K-AA-611 & $02 \times 7-12-A$ & U234 & 9.72E-04 & 2.12E-04 & 4.42E-05 & 4.6 \\
\hline K-AA-611 & $02 \times 7-12-A$ & U238 & $6.10 E-04$ & $1.55 \mathrm{E}-04$ & 5.05E-05 & 3.9 \\
\hline K-AA-612 & $02 \times 7-13-A$ & U234 & $9.21 \mathrm{E}-04$ & 2.06E-04 & 4.45E-05 & 4.5 \\
\hline K-AA-612 & $02 \times 7-13-A$ & U238 & $1.09 E-03$ & 2.29E-04 & 5.09E-05 & 4.8 \\
\hline K-AA-614 & $02 \times 7-15-A$ & U234 & 1.47E-03 & 2.69E-04 & 4.58E-05 & 5.5 \\
\hline K-AA-614 & $02 \times 7-15-A$ & U238 & 1.43E-03 & 2.72E-04 & 5.07E-05 & 5.3 \\
\hline K-AA-615 & $02 \times 7-16-A$ & U234 & 1.04E-03 & $2.22 E-04$ & $3.04 E-05$ & 4.7 \\
\hline K-AA-615 & $02 \times 7-16-A$ & U238 & 7.67E-04 & 1.79E-04 & $3.63 E-05$ & 4.3 \\
\hline K-AA-616 & $02 \times 7-17-A$ & U234 & 1.25E-03 & 2.43E-04 & 4.84E-05 & 5.1 \\
\hline K-AA-616 & $02 \times 7-17-A$ & U235 & $9.38 E-05$ & $4.35 E-05$ & $5.05 E-05$ & 2.2 \\
\hline K-AA-616 & $02 \times 7-17-A$ & U238 & $9.79 E-04$ & $2.06 \mathrm{E}-04$ & $3.26 \mathrm{E}-05$ & 4.8 \\
\hline K-AA- 617 & $02 \times 7-18-A$ & U234 & $1.53 E-03$ & 2.69E-04 & $3.68 E-05$ & 5.7 \\
\hline K-AA- 617 & $02 \times 7-18-A$ & U238 & 1.36E-03 & 2.57E-04 & $3.37 E-05$ & 5.3 \\
\hline K-AA-618 & $02 \times 7-19-A$ & U234 & 2.19E-03 & 5.71E-04 & 1.36E-04 & 3.8 \\
\hline K-AA- 618 & $02 \times 7-19-A$ & U238 & $1.29 \mathrm{E}-03$ & $3.97 E-04$ & $2.02 E-04$ & 3.2 \\
\hline K-AA-619 & $02 \times 7-20-A$ & PU238 & $1.04 E-02$ & 1.17E-03 & $7.38 E-05$ & 8.9 \\
\hline K-AA-619 & $02 \times 7-20-A$ & PU239/240 & $9.10 \mathrm{E}-03$ & $1.04 E-03$ & 6.87E-05 & 8.8 \\
\hline K-AA-619 & $02 \times 7-20-A$ & U234 & 6.37E-03 & $9.12 E-04$ & $7.49 E-05$ & 7.0 \\
\hline K-AA-619 & $02 \times 7-20-A$ & U235 & 2.96E-04 & 9.38E-05 & 6.35E-05 & 3.2 \\
\hline K-AA-619 & $02 \times 7-20-A$ & U238 & 6.64E-03 & $1.03 E-03$ & 6.21E-05 & 6.4 \\
\hline K-BB-600 & $02 \times 7-01-A$ & U234 & 3.95E-04 & 1.36E-04 & $4.29 E-05$ & 2.9 \\
\hline K-BB-600 & $02 \times 7-01-A$ & U238 & 3.05E-04 & 1.06E-04 & 4.61E-05 & 2.9 \\
\hline
\end{tabular}




\section{Alpha Analysis for Amchitka Island (Batch 14) Summary of 2 and 3 sigma activities}

page 2

\begin{tabular}{|c|c|c|c|c|c|c|}
\hline $\begin{array}{l}\text { Customer } \\
\text { ID }\end{array}$ & Lab ID & Isotope & $\begin{array}{c}\text { Result } \\
\mathrm{Bq} / \mathrm{g}\end{array}$ & $\begin{array}{c}\text { Uncertainty } \\
\mathrm{Bq} / \mathrm{g}\end{array}$ & MDA & $\begin{array}{c}\text { Result/ } \\
\text { Uncertainty }\end{array}$ \\
\hline K-BB-601 & $02 \times 7-02-A$ & U234 & $1.09 \mathrm{E}-03$ & 2.23E-04 & $3.32 \mathrm{E}-05$ & 4.9 \\
\hline K-BB-601 & $02 \times 7-02-A$ & U238 & 9.99E-04 & $2.10 E-04$ & 3.74E-05 & 4.8 \\
\hline K-BB-602 & $02 \times 7-03-A$ & U234 & 1.17E-03 & 2.35E-04 & 5.28E-05 & 5.0 \\
\hline K-BB-602 & 02X7-03-A & U238 & $1.25 \mathrm{E}-03$ & $2.48 \mathrm{E}-04$ & 4.18E-05 & 5.0 \\
\hline K-BB-603 & $02 \times 7-04-A$ & U234 & $4.23 E-04$ & $1.40 E-04$ & 4.33E-05 & 3.0 \\
\hline K-BB-603 & $02 \times 7-04-A$ & U238 & $4.50 E-04$ & $1.29 \mathrm{E}-04$ & 3.95E-05 & 3.5 \\
\hline K-BB-604 & $02 \times 7-05-A$ & U234 & $1.12 E-03$ & 2.29E-04 & $3.41 E-05$ & 4.9 \\
\hline K-BB-604 & $02 \times 7-05-A$ & U238 & 9.33E-04 & 2.02E-04 & 4.80E-05 & 4.6 \\
\hline K-BB-605 & $02 \times 7-06-A$ & U234 & $1.08 \mathrm{E}-03$ & 2.24E-04 & $4.55 \mathrm{E}-05$ & 4.8 \\
\hline K-BB-605 & $02 \times 7-06-A$ & U235 & 8.98E-05 & $4.40 \mathrm{E}-05$ & 5.73E-05 & 2.0 \\
\hline K-BB-605 & $02 \times 7-06-A$ & U238 & $9.69 E-04$ & 2.07E-04 & 4.83E-05 & 4.7 \\
\hline K-BB-606 & 02X7-07-A & U234 & $2.06 \mathrm{E}-03$ & 3.51E-04 & 3.01E-05 & 5.9 \\
\hline K-BB-606 & $02 \times 7-07-A$ & U235 & $1.60 E-04$ & $5.81 E-05$ & 5.99E-05 & 2.8 \\
\hline K-BB-606 & $02 \times 7-07-A$ & U238 & $2.11 E-03$ & 3.77E-04 & 3.59E-05 & 5.6 \\
\hline K-BB-607 & $02 \times 7-08-A$ & U234 & $9.89 E-04$ & $2.11 E-04$ & 4.71E-05 & 4.7 \\
\hline K-BB-607 & $02 \times 7-08-A$ & U238 & 8.67E-04 & $1.91 \mathrm{E}-04$ & 5.21E-05 & 4.5 \\
\hline K-BB-608 & $02 \times 7-09-A$ & PU239/240 & 1.03E-04 & 5.16E-05 & 6.84E-05 & 2.0 \\
\hline K-BB-608 & $02 \times 7-09-A$ & U234 & 7.71E-04 & 1.84E-04 & 5.36E-05 & 4.2 \\
\hline K-BB-608 & $02 \times 7-09-A$ & U238 & 7.71E-04 & 1.77E-04 & 4.24E-05 & 4.4 \\
\hline K-BB-609 & $02 \times 7-10-A$ & U234 & $7.64 E-03$ & $1.00 \mathrm{E}-03$ & 4.73E-05 & 7.6 \\
\hline K-BB-609 & $02 \times 7-10-A$ & U235 & 4.47E-04 & $1.06 E-04$ & 5.96E-05 & 4.2 \\
\hline K-BB-609 & $02 \times 7-10-A$ & U238 & 7.49E-03 & $1.09 E-03$ & 3.57E-05 & 6.9 \\
\hline
\end{tabular}

All known sources of uncertainty are included in the uncertainty term. There may be unknown sources of uncertainty that are not accounted for. If the result/uncertainty ratio is more than 3, we have a degree of confidence that the result is positive (i.e. the result is statistically different than zero). A result with the resultuncertainty ratio between 2 and 3 is the first indication that an isotope may be present and further investigation may be warranted.

As with any good science no single data point is used in important decisions (results need to be reproducible). 


\section{RADIOANALYTICAL ANALYSES DATA PACKAGE}

Project Title: $\quad$ Amchitka Island Alpha Analysis Report (Batch 15)

Lab Name:

RTC

Case No: NA

Report No.

AmchitBatchF15

Method Type: A/B

Approved SAP No:: NA

SDG No.: $\quad$ K-EE-620

\section{SAMPLE NUMBERS}

Customer

Sample ID

\begin{tabular}{|c|}
\hline K-EE-620 \\
\hline K-EE-621 \\
\hline K-EE-622 \\
\hline K-EE-623 \\
\hline K-EE-624 \\
\hline K-EE-625 \\
\hline K-EE-626 \\
\hline K-EE-627 \\
\hline K-EE-628 \\
\hline K-EE-638 \\
\hline K-FF- 629 \\
\hline K-GG-630 \\
\hline K-GG-631 \\
\hline K-GG-632 \\
\hline K-GG-633 \\
\hline K-GG-634 \\
\hline $\mathrm{K}-\mathrm{HH}-635$ \\
\hline $\mathrm{K}-\mathrm{HH}-636$ \\
\hline $\mathrm{K}-\mathrm{HH}-637$ \\
\hline $\mathrm{K}-\mathrm{HH}-639$ \\
\hline
\end{tabular}

Lab Sample

ID

\begin{tabular}{|c|}
\hline 02XP-01-A \\
\hline $02 \times P-02-A$ \\
\hline 02XP-03-A \\
\hline 02XP-04-A \\
\hline $02 \times P-05-A$ \\
\hline 02XP-06-A \\
\hline $02 \times P-07-A$ \\
\hline 02XP-08-A \\
\hline 02XP-09-A \\
\hline $02 \times P-19-A$ \\
\hline $02 \times P-10-A$ \\
\hline 02XP-11-A \\
\hline $02 \times P-12-A$ \\
\hline $02 \times P-13-A$ \\
\hline $02 \times P-14-A$ \\
\hline $02 \times P-15-A$ \\
\hline $02 \times P-16-A$ \\
\hline $02 \times P-17-A$ \\
\hline $02 \times P-18-A$ \\
\hline 02XP-20-A \\
\hline
\end{tabular}

Comments:

Release of the data contained in this data package has been authorized by the laboratory manager or the manager's designee, as verified by the following signature:

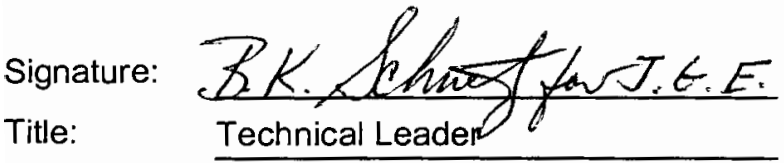

Date: $\quad 11 / 22 / 2005$ 


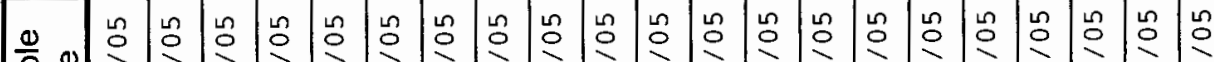

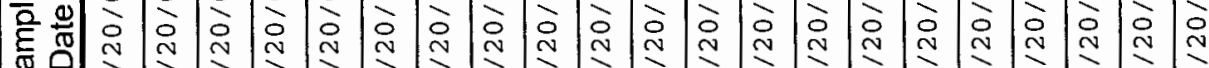

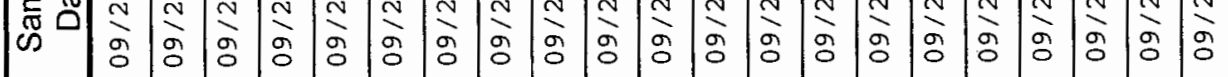

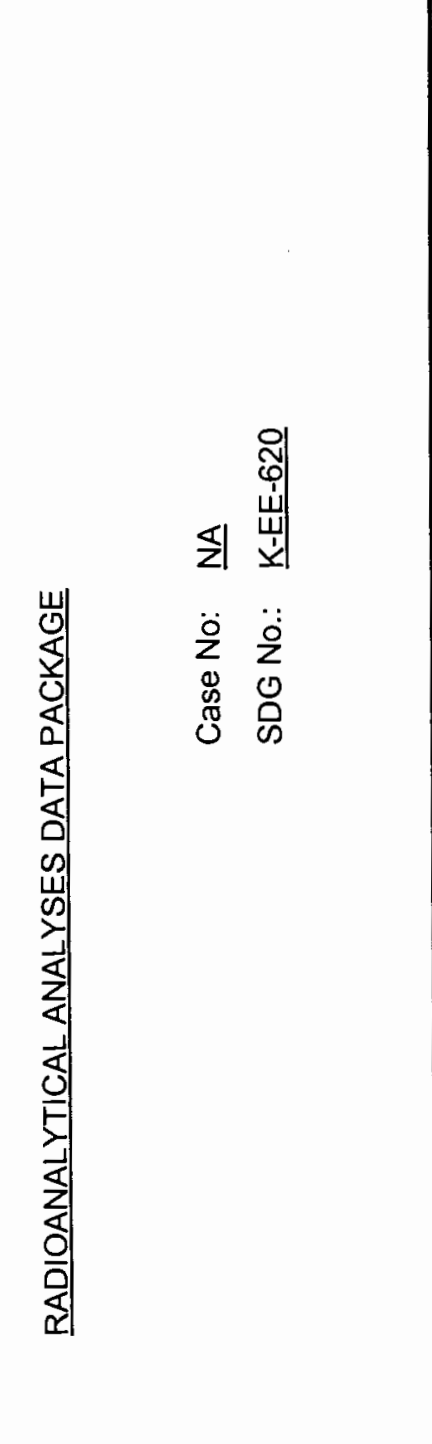

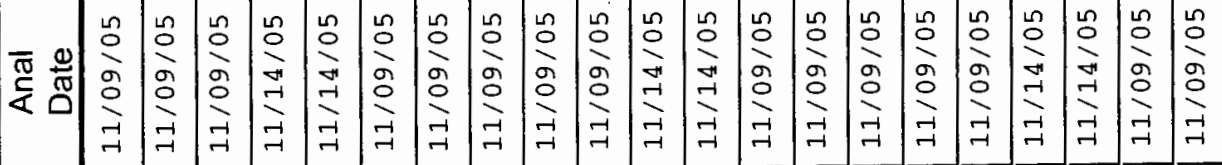
空

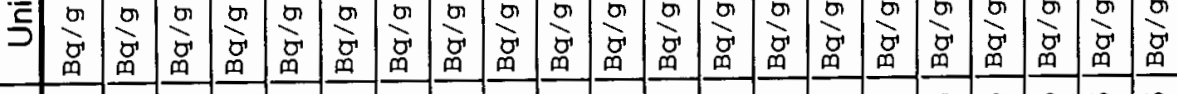

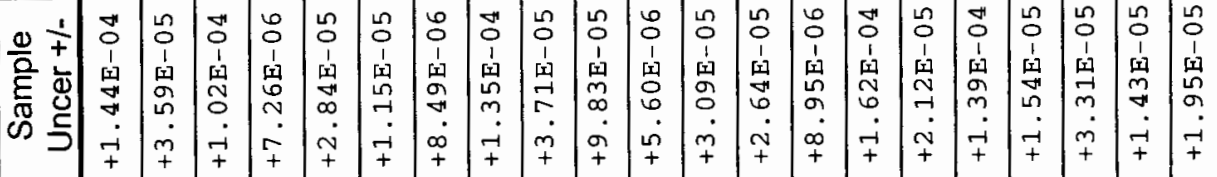

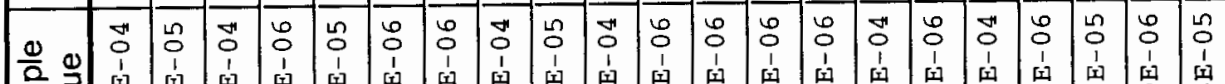

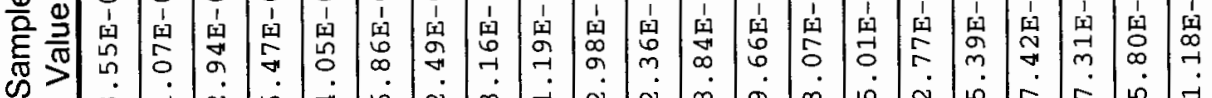

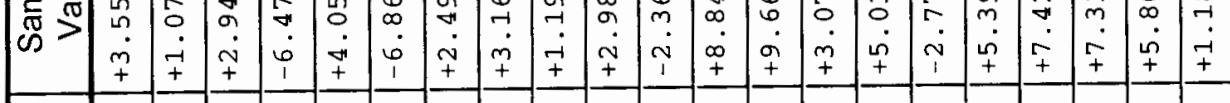

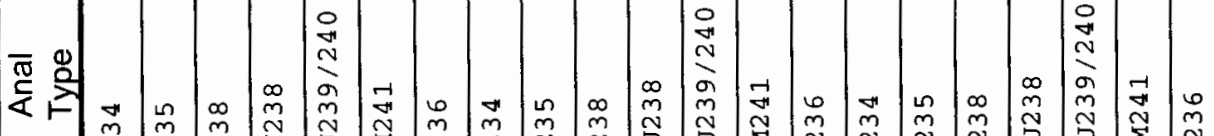

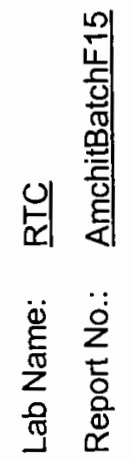

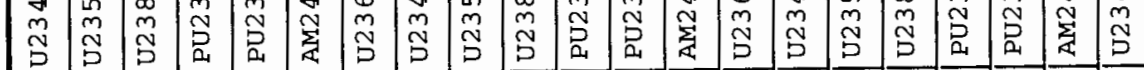

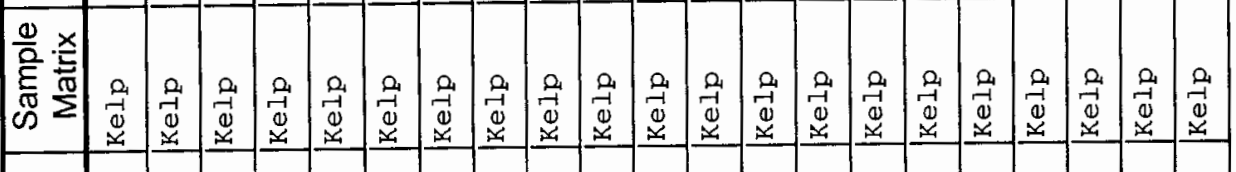

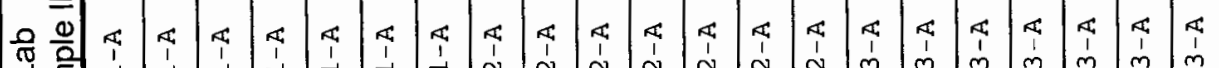

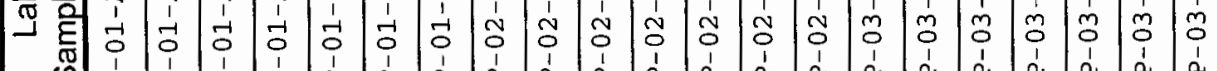

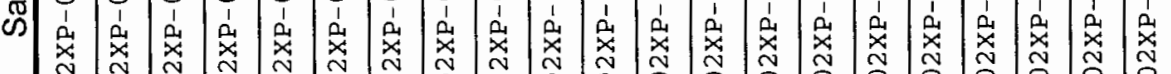

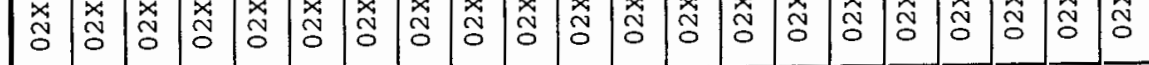




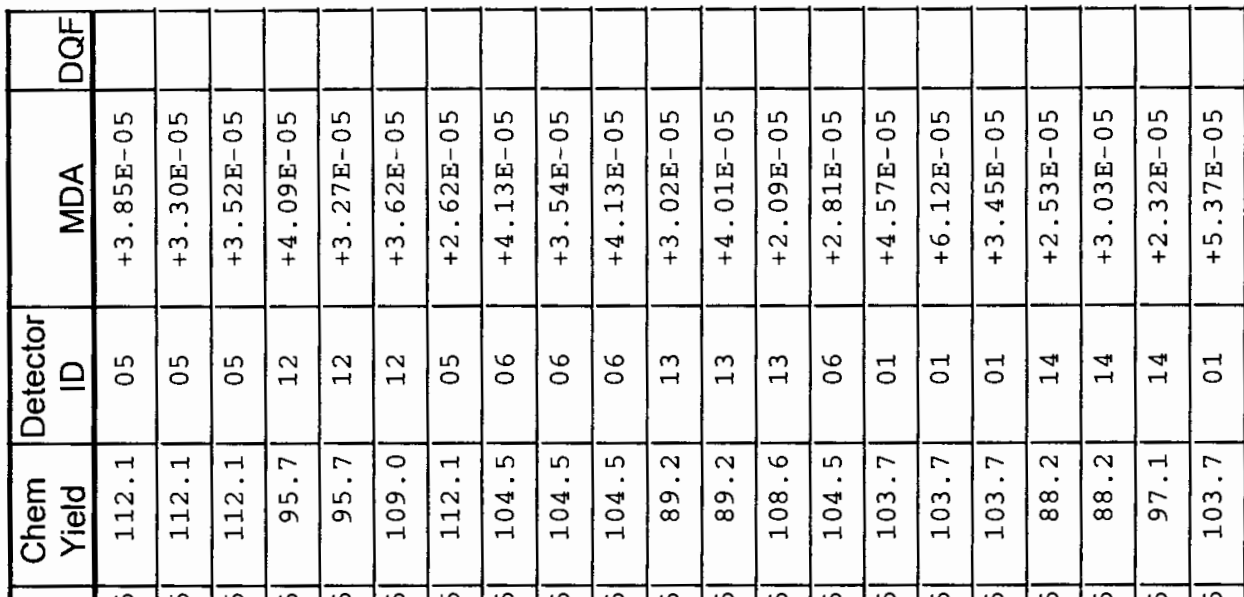
送

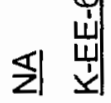

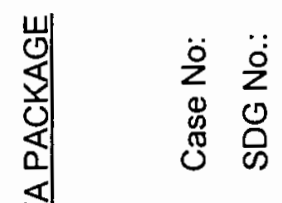

电

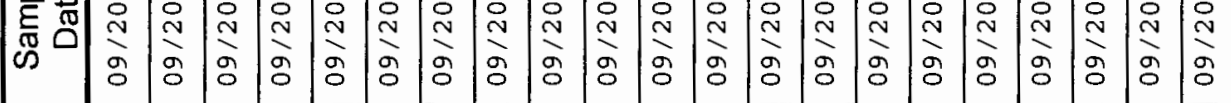

|ct

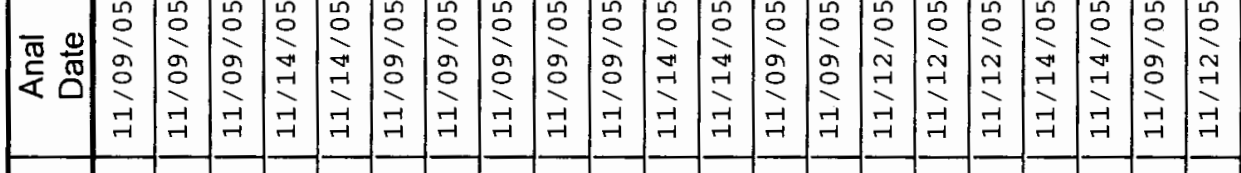

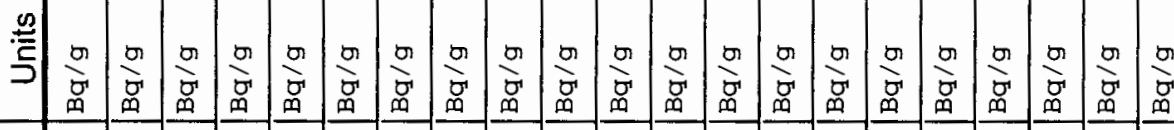

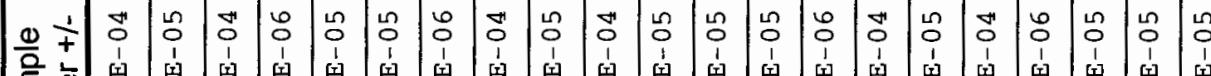
密

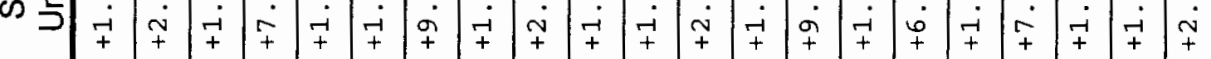

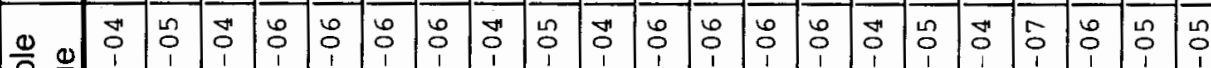

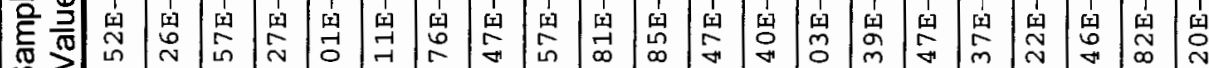
趈>

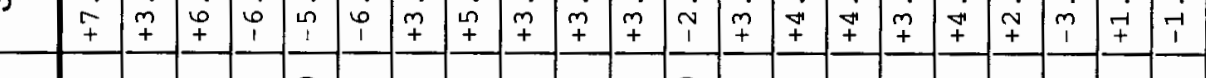

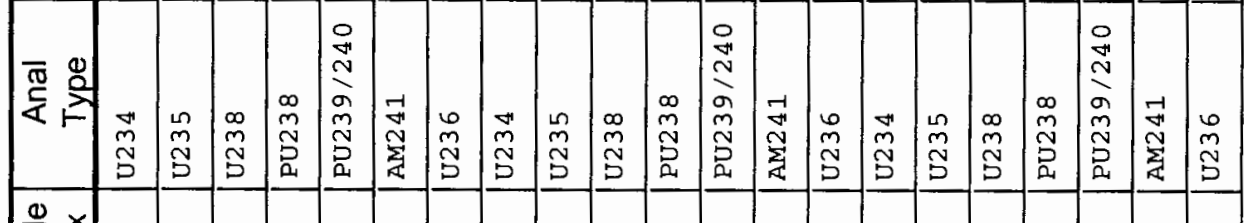
임

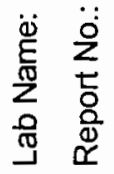
每

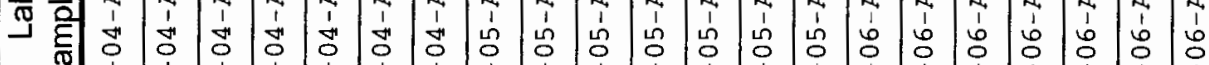

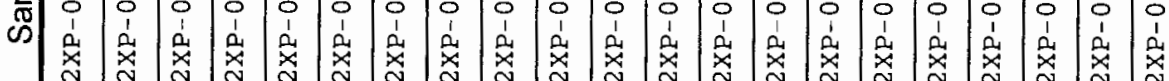

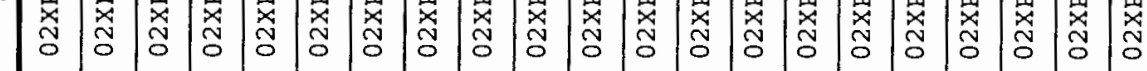

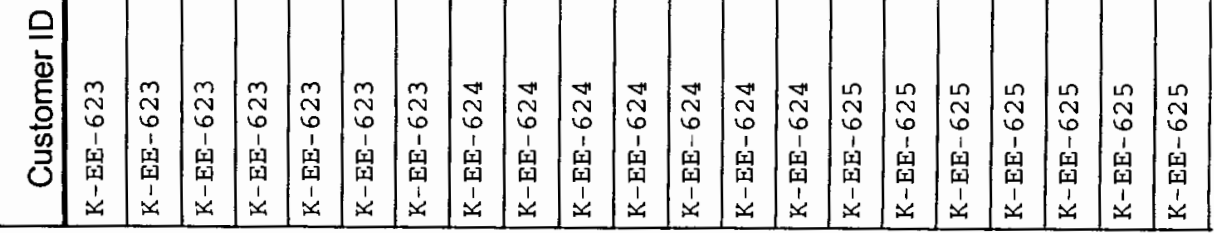




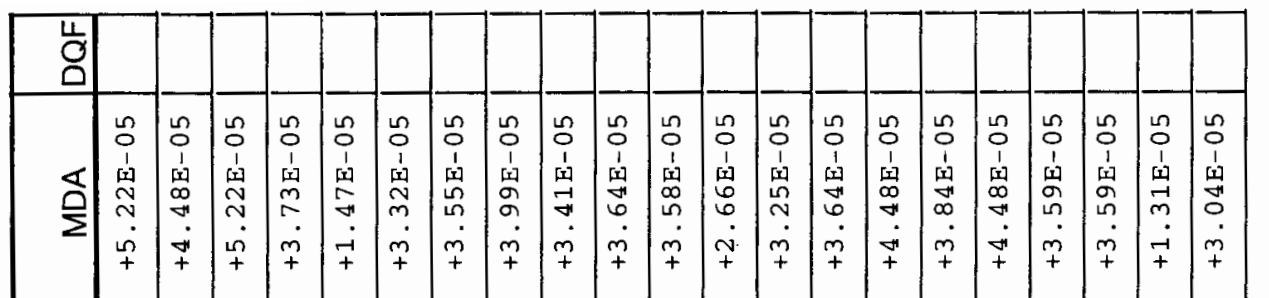

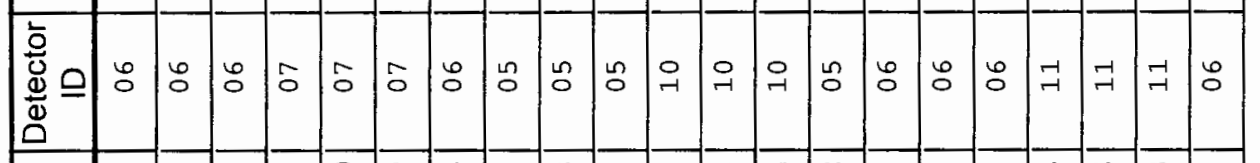

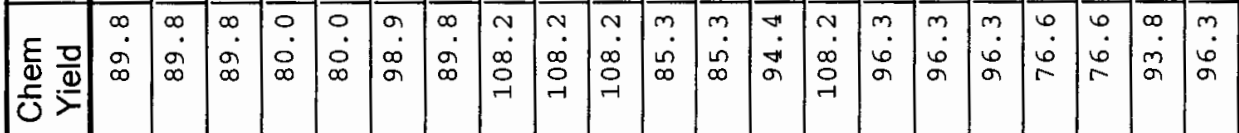

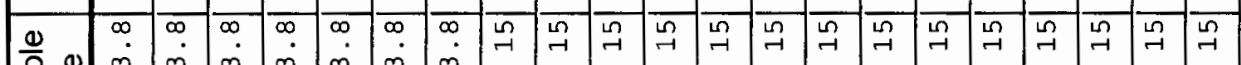

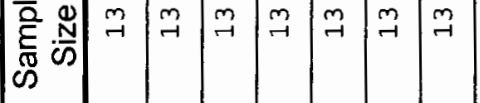

艺

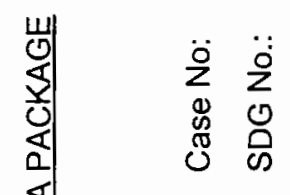

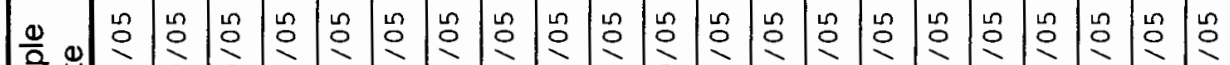

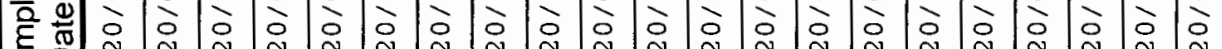

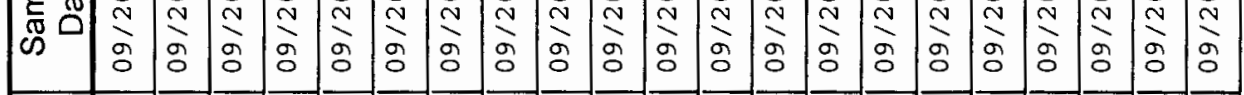
刃⿻

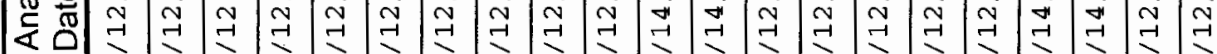

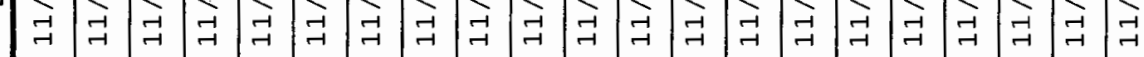

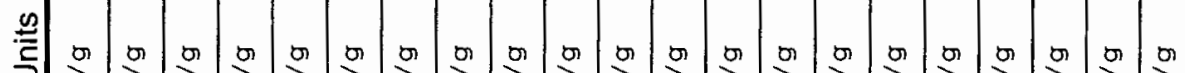

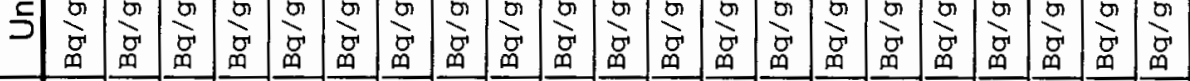

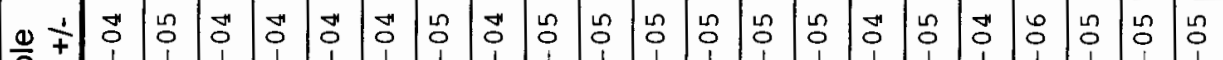

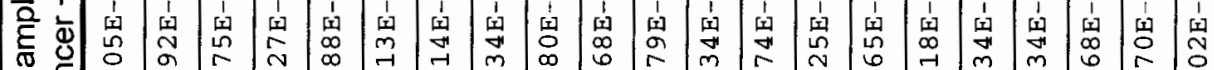

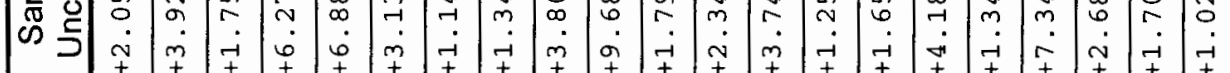

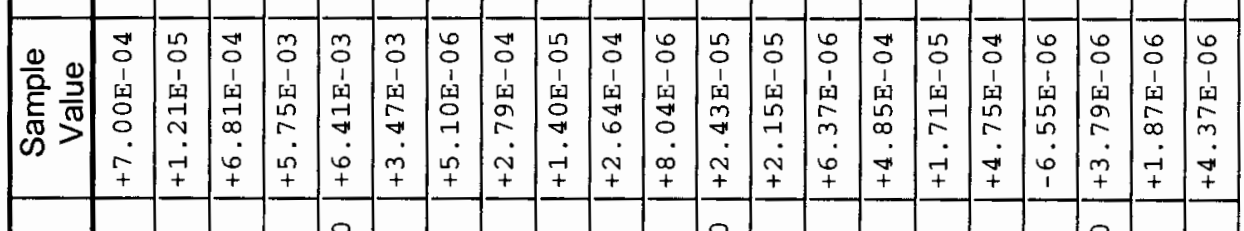

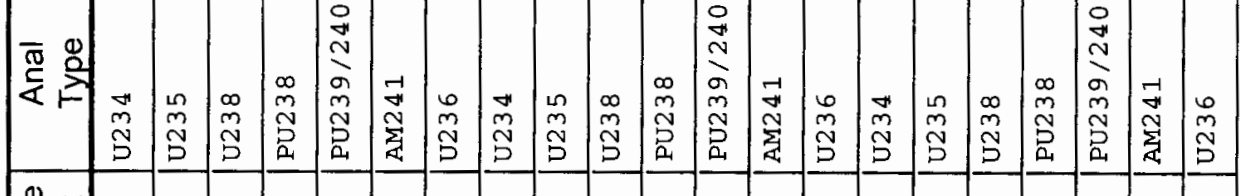
임

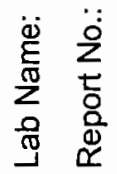

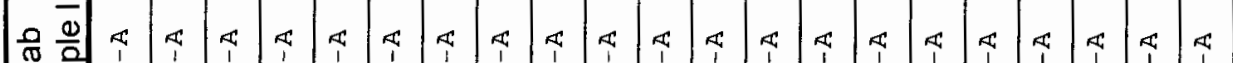

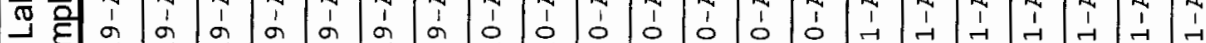

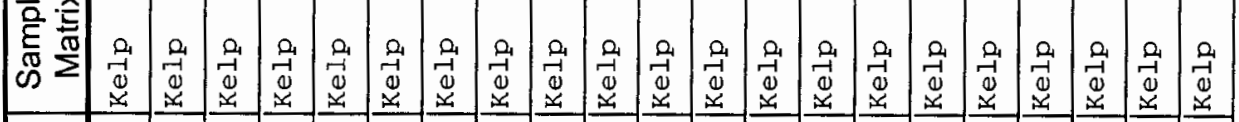

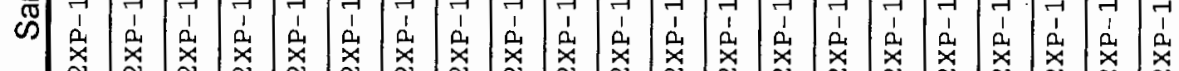

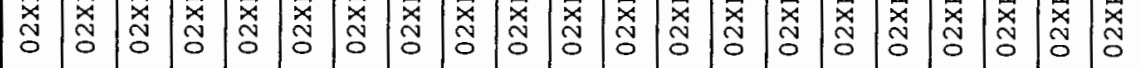

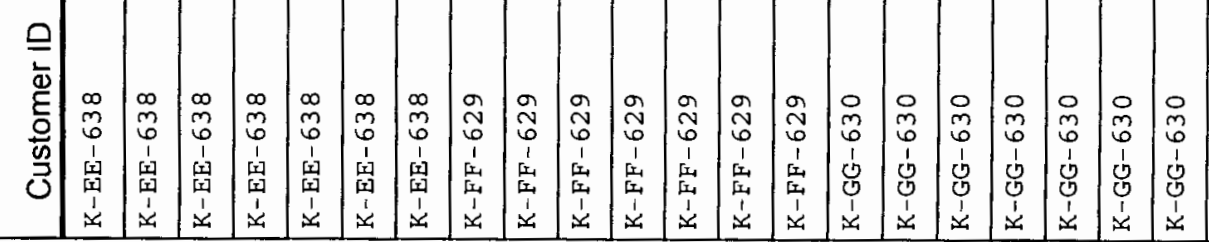



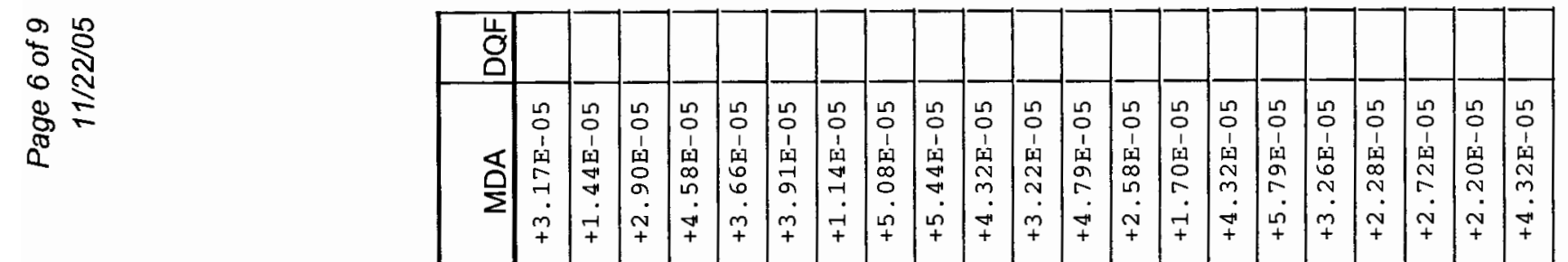

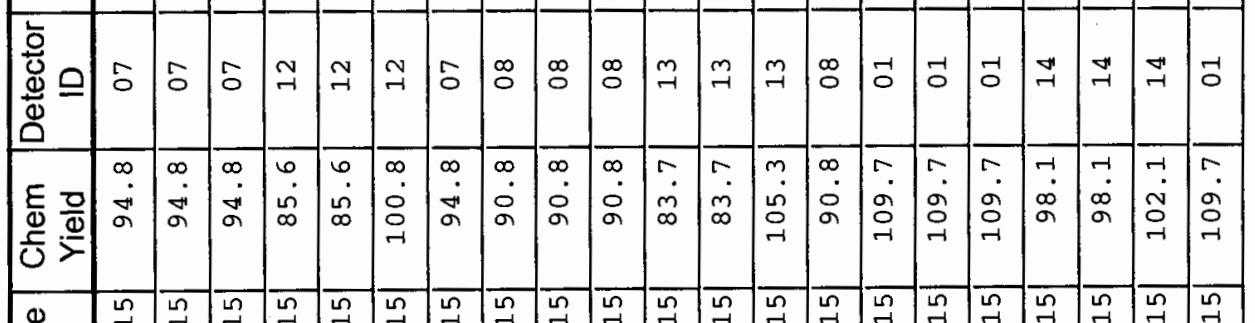
元 多 岁|

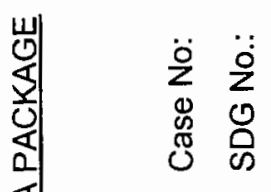

里

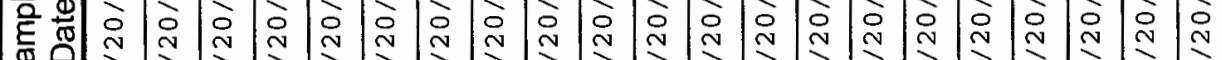

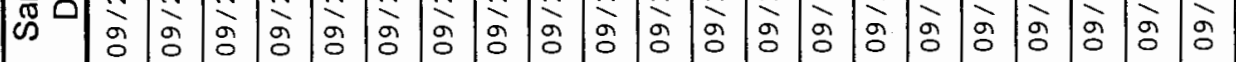

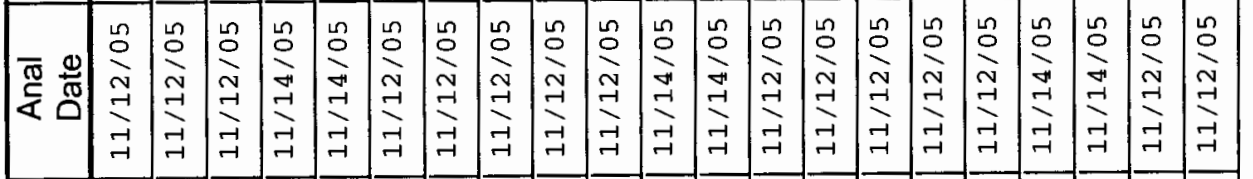

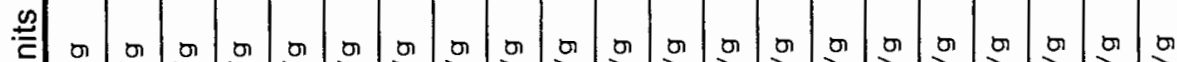

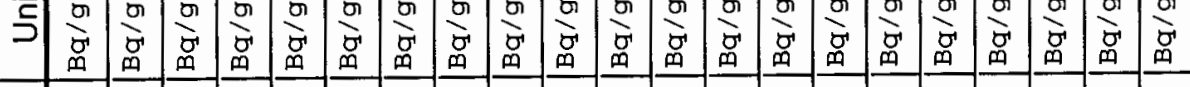
થ)

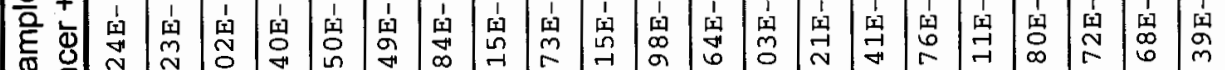
œ

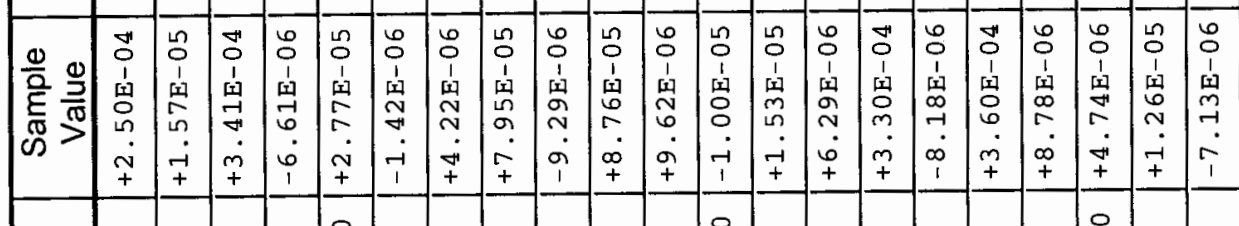
可

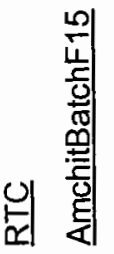

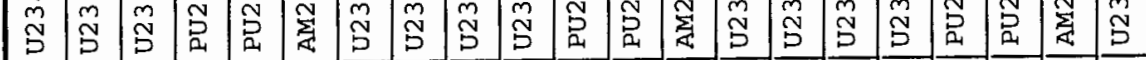

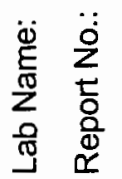

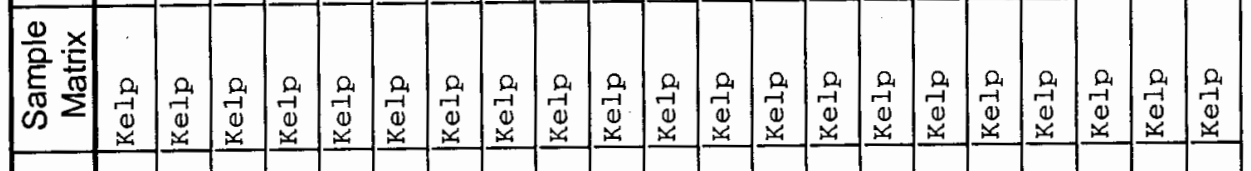

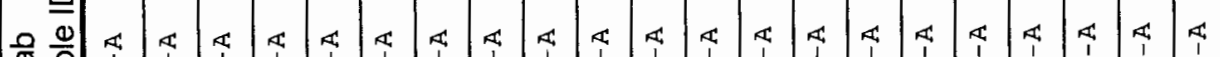
苞

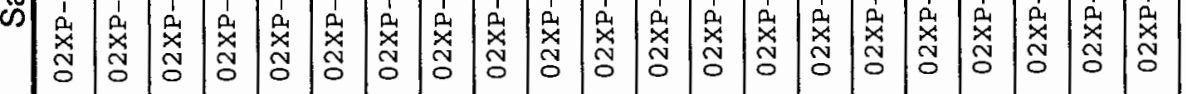

으

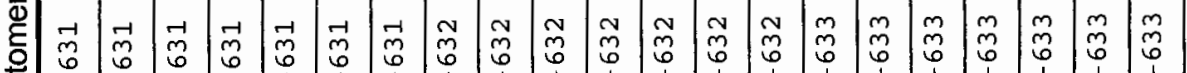




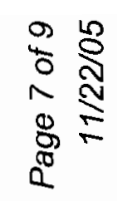

\begin{tabular}{|c|c|c|c|c|c|c|c|c|c|c|c|c|}
\hline & & & & & & & & & & & & \\
\hline & 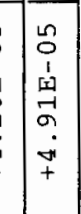 & & & & 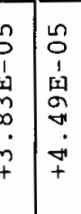 & & 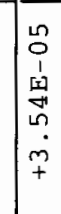 & & & & & \\
\hline & & & & & & & & & & & & \\
\hline & & & & & & & & & & & & \\
\hline
\end{tabular}

约岗

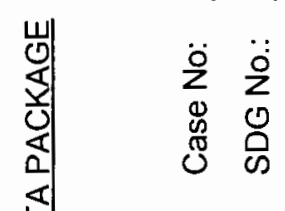

量语

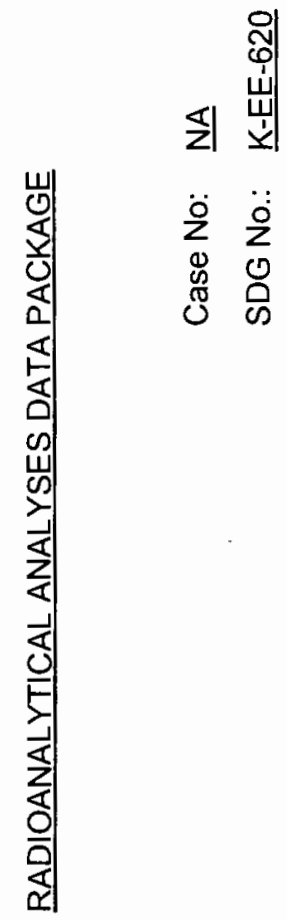

橆 它

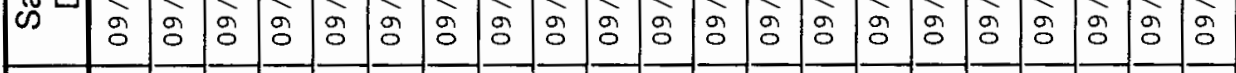

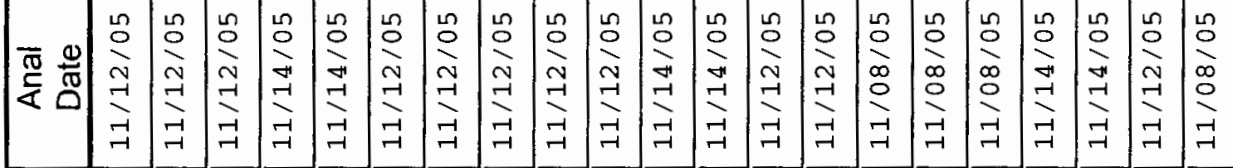

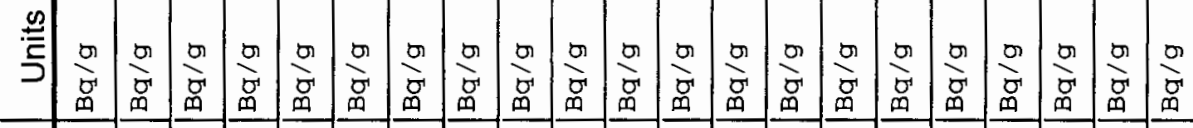

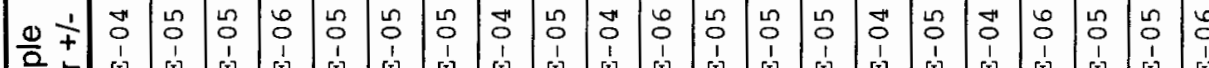

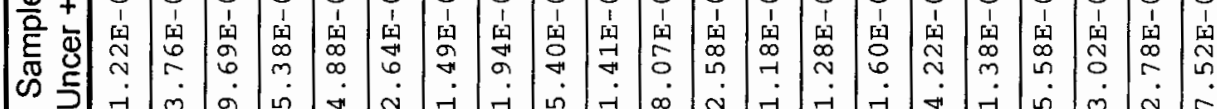

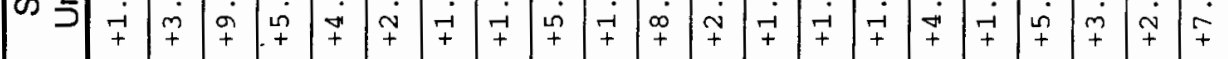

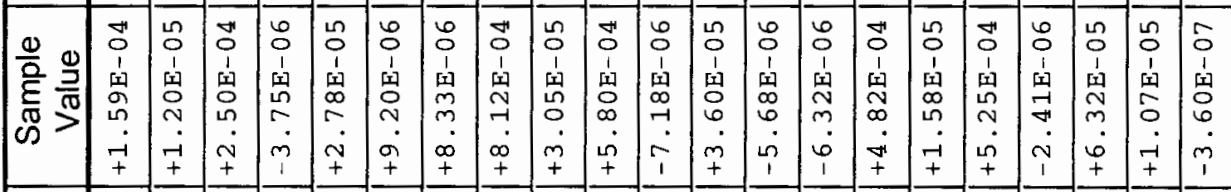

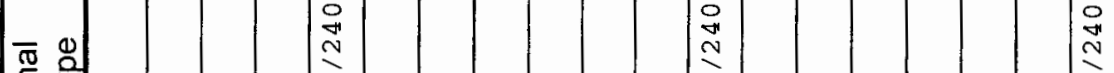

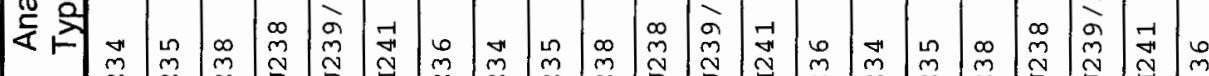

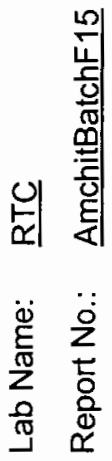

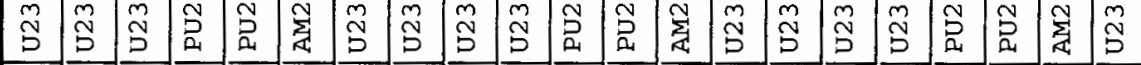

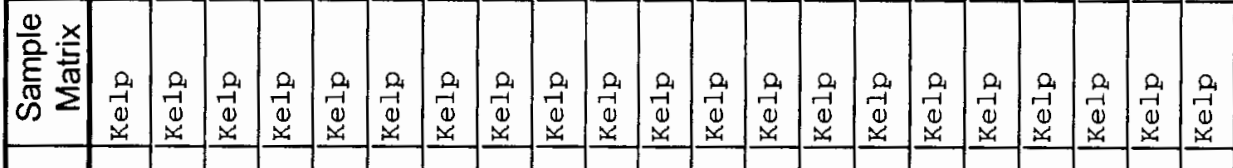
远

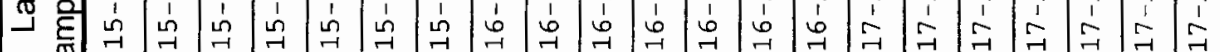

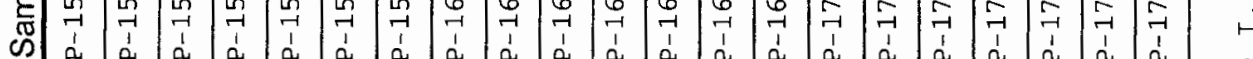

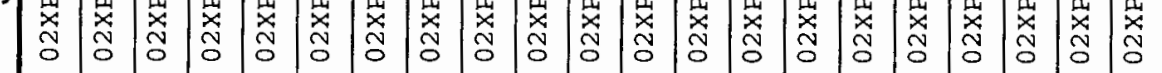

은

惫 焉 


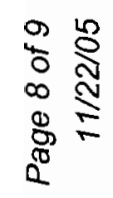

\begin{tabular}{|c|c|c|c|c|c|c|c|c|c|c|c|c|c|c|}
\hline $\begin{array}{l} \\
\end{array}$ & & & & & & & & & & & & & & \\
\hline \begin{tabular}{l}
\multicolumn{1}{c}{} \\
¿ \\
$\Sigma$
\end{tabular} & 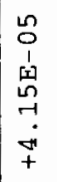 & $\begin{array}{l}n \\
0 \\
1 \\
1 \\
0 \\
0 \\
0 \\
0 \\
\dot{m} \\
+\end{array}$ & $\begin{array}{l}n \\
0 \\
1 \\
1 \\
5 \\
2 \\
5 \\
m \\
+\end{array}$ & 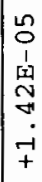 & 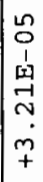 & \begin{tabular}{l}
$n$ \\
0 \\
1 \\
1 \\
0 \\
0 \\
\hdashline \\
$p$ \\
+
\end{tabular} & $\begin{array}{l}n \\
0 \\
1 \\
1 \\
5 \\
\vdots \\
0 \\
0 \\
+\end{array}$ & 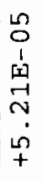 & 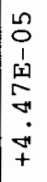 & 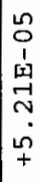 & $\begin{array}{l}\vec{D} \\
0 \\
1 \\
1 \\
\\
N \\
-1 \\
+ \\
+\end{array}$ & $\begin{array}{l}\overrightarrow{0} \\
0 \\
1 \\
1 \\
y \\
-1 \\
-1 \\
+ \\
+\end{array}$ & $\begin{array}{l}\text { Dี } \\
1 \\
1 \\
0 \\
-1 \\
-1 \\
+ \\
+\end{array}$ & 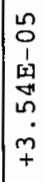 \\
\hline 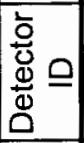 & $\stackrel{\sim}{\circ}$ & 告 & 10 & $\exists$ & $\exists$ & - & $\stackrel{2}{\circ}$ & $\because$ & $1 \%$ & $\because 0$ & ${ }_{0}^{\infty}$ & $\stackrel{\infty}{\circ}$ & $\stackrel{\infty}{\circ}$ & 10 \\
\hline$\frac{E}{\Phi} \frac{0}{0}$ & $\begin{array}{l}\dot{\sigma} \\
\dot{m} \\
0 \\
\sigma\end{array}$ & $\begin{array}{l}\vec{\rho} \\
\dot{m} \\
\dot{0} \\
-1\end{array}$ & 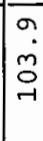 & $\begin{array}{l}\overrightarrow{9} . \\
\dot{n} \\
\infty\end{array}$ & $\begin{array}{l}\bar{\sigma} \\
\dot{\infty} \\
\infty\end{array}$ & $\begin{array}{l}-1 \\
\dot{\circ} \\
\sigma\end{array}$ & $\begin{array}{l}\rho \\
\dot{m} \\
0 \\
-1\end{array}$ & $\begin{array}{l}7 . \\
\dot{0} \\
\sigma \\
\sigma\end{array}$ & $\begin{array}{l}7 . \\
\infty \\
\sigma \\
\sigma\end{array}$ & $\begin{array}{l}7 \\
\infty \\
0 \\
\sigma\end{array}$ & $\dot{\pi}$ & i & $\begin{array}{l}0 \\
\dot{0} \\
\infty\end{array}$ & $\begin{array}{l}-1 \\
\infty \\
\infty\end{array}$ \\
\hline 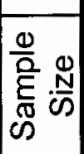 & $\begin{array}{ll}10 \\
\sim 1\end{array}$ & $\stackrel{\sim}{\rightarrow}$ & $\cong$ & $\stackrel{\sim}{\sim}$ & $\stackrel{\sim}{\sim}$ & I & $\begin{array}{l}n \\
r-1\end{array}$ & $\begin{array}{l}6 \\
\dot{H} \\
\vec{H}\end{array}$ & $\begin{array}{l}\overrightarrow{6} \\
\dot{i}\end{array}$ & $\begin{array}{l}\overline{0} \\
\dot{\sim}\end{array}$ & $\begin{array}{l}\overline{0} \\
\dot{\mathcal{H}}\end{array}$ & 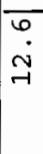 & $\underset{F}{F}$ & $\begin{array}{l}\overrightarrow{6} \\
\text { ने }\end{array}$ \\
\hline
\end{tabular}

飞| 위ํ|

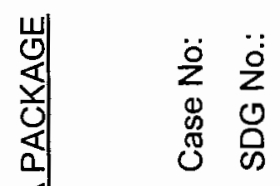

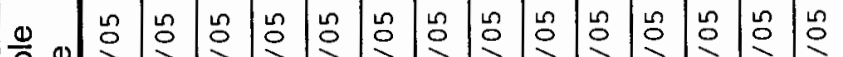

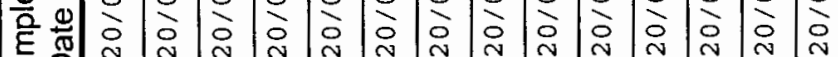

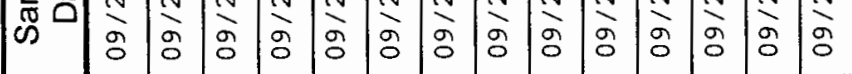

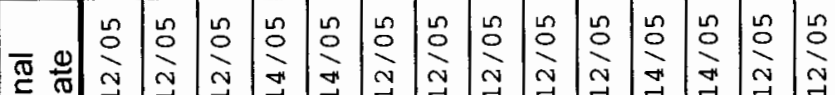

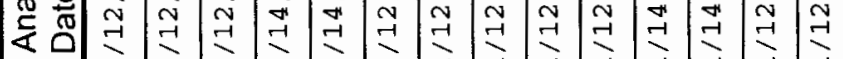

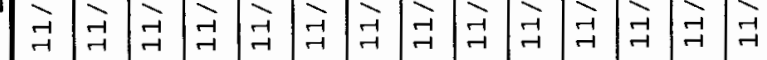

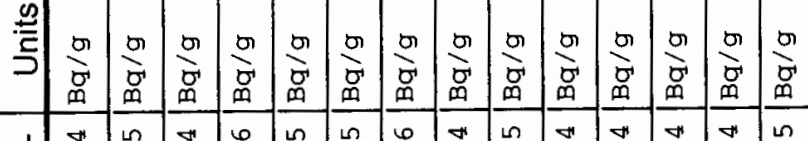

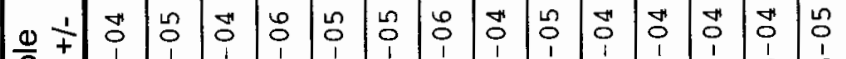

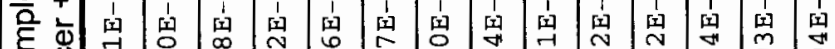

ल

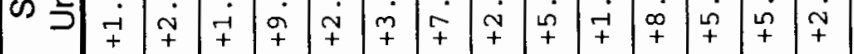

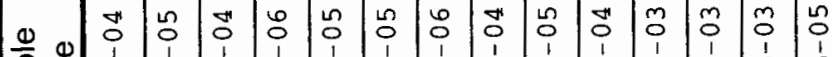

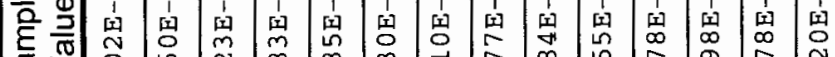

n

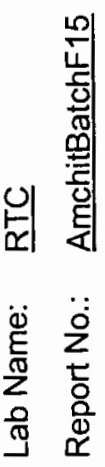

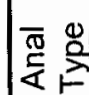

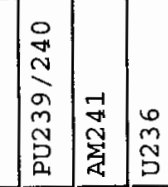

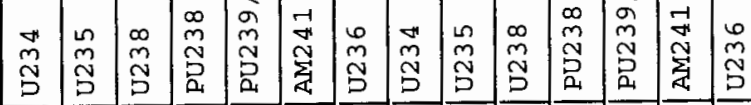

\begin{tabular}{|c|c|c|c|c|c|c|c|c|c|c|c|c|c|c|}
\hline 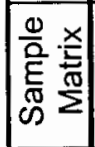 & 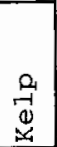 & 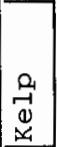 & 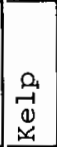 & $\begin{array}{l}a_{4} \\
0 \\
0 \\
\vdots \\
\vdots\end{array}$ & \begin{tabular}{|l}
0 \\
$\overrightarrow{0}$ \\
$\dot{0}$
\end{tabular} & $\begin{array}{l}a_{1} \\
0 \\
\ddot{1}\end{array}$ & 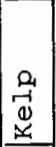 & $\begin{array}{l}0 \\
-1 \\
0 \\
0\end{array}$ & 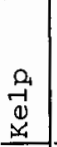 & $\begin{array}{l}a_{4} \\
\vec{d} \\
\ddot{x}\end{array}$ & 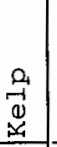 & 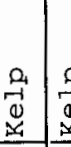 & 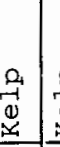 & \begin{tabular}{l}
0 \\
$\vec{y}$ \\
$\vec{y}$ \\
\cline { 1 - 1 }
\end{tabular} \\
\hline 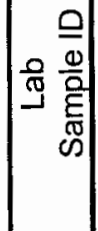 & 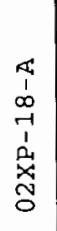 & 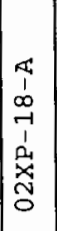 & 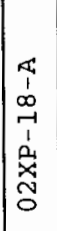 & 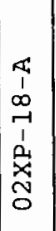 & 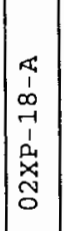 & 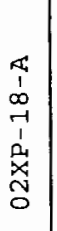 & $\mid$\begin{tabular}{c}
1 \\
1 \\
0 \\
0 \\
-1 \\
1 \\
$a$ \\
\multirow{x}{*}{} \\
0 \\
0
\end{tabular} & 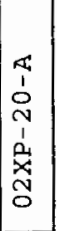 & 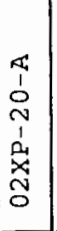 & 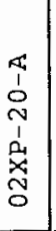 & 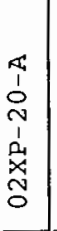 & 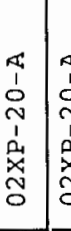 & 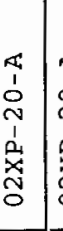 & 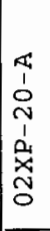 \\
\hline 苞 & 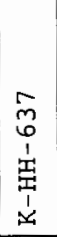 & 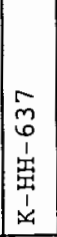 & 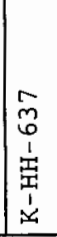 & 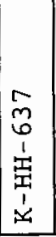 & 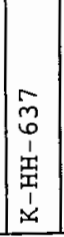 & 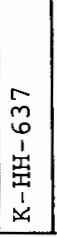 & 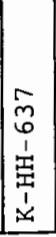 & 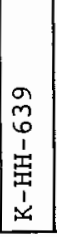 & 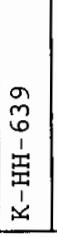 & 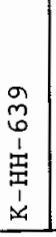 & 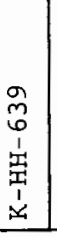 & 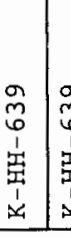 & 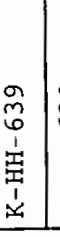 & 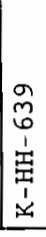 \\
\hline
\end{tabular}


2
0
0
0
0
0
0
0
0

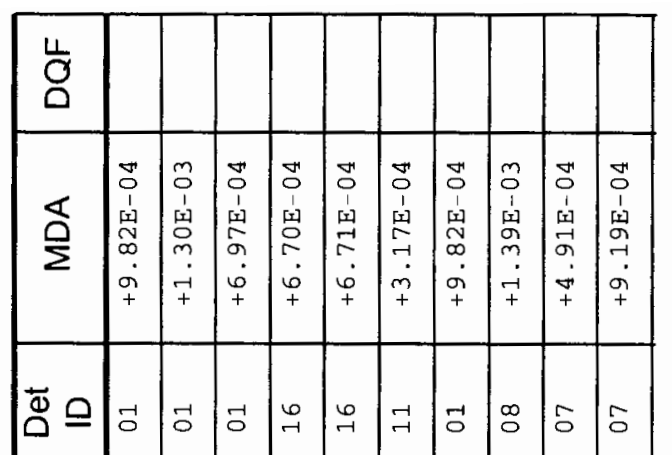

완 원

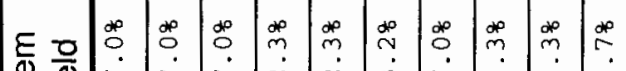

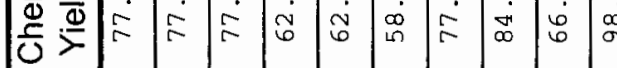

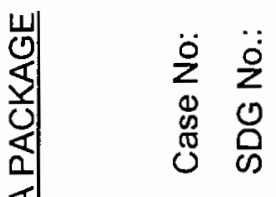

匹

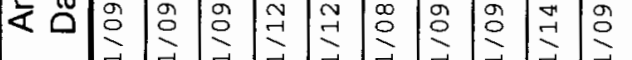

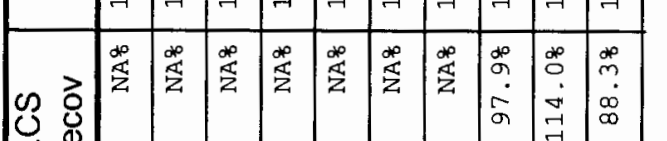

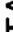

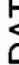

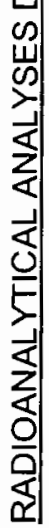

$\checkmark \mathbb{x}$

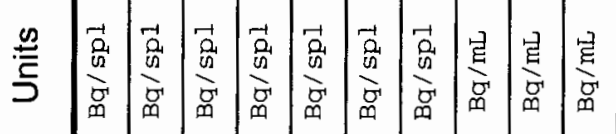

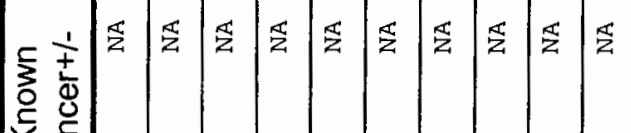

$\times 5$

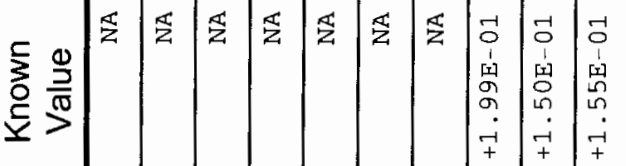

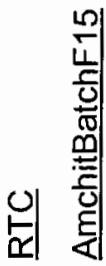

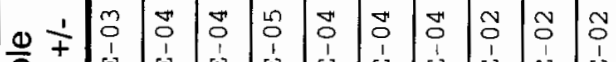

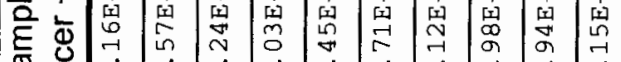

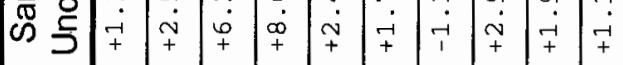

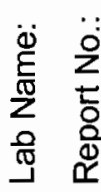

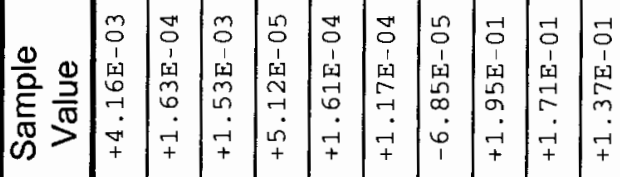

\begin{tabular}{|c|c|c|c|c|c|c|c|c|c|c|}
\hline$\frac{\stackrel{\Xi}{\Xi}}{\frac{\Xi}{\Sigma}}$ & $\underset{\stackrel{\sim}{S}}{\stackrel{\leftrightarrow}{S}}$ & 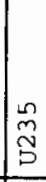 & 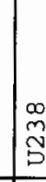 & 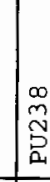 & 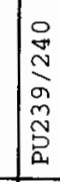 & $\mid \begin{array}{l}\vec{N} \\
\frac{\pi}{2}\end{array}$ & $\begin{array}{l}\infty \\
\underset{N}{S} \\
\end{array}$ & $\mid \begin{array}{l}\infty \\
m \\
\end{array}$ & 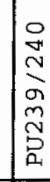 & $\mid \begin{array}{l}\vec{F} \\
\widetilde{N} \\
\text { S }\end{array}$ \\
\hline 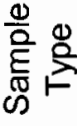 & $\frac{\vec{c}}{m}$ & 岕 & 䓌 & 苟 & 琶 & 扂 & 荺 & $\begin{array}{l}0 \\
U \\
U\end{array}$ & 苞 & 吕 \\
\hline 旡 & $\sum_{i=1}^{E}$ & $\begin{array}{l}\mathbf{E} \\
\text { 舄 }\end{array}$ & 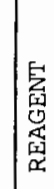 & 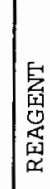 & 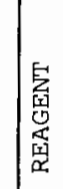 & 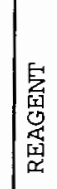 & 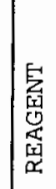 & 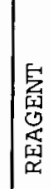 & 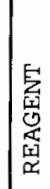 & 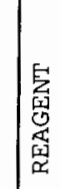 \\
\hline
\end{tabular}



Project: Alpha Analysis for Amchitka Island (Batch 15)

Laboratory: RTC

Report \#: AmchitBatchF15

SDG\#: $\quad$ K-EE-620

\section{Summary of 2 and 3 sigma activities}

Below are the results for U234, U235, U236, U238, Pu238, Pu239/240, and Am241 for Batch 13 from the Amchitka Island Project that had a result/uncertainty ratio of 2 or more (uncertainty@onesigma).

\begin{tabular}{|c|c|c|c|c|c|c|}
\hline $\begin{array}{l}\text { Customer } \\
\text { ID }\end{array}$ & Lab ID & Isotope & $\begin{array}{c}\text { Result } \\
\mathrm{Bq} / \mathrm{g}\end{array}$ & $\begin{array}{c}\text { Uncertainty } \\
\text { Bq/g }\end{array}$ & MDA & $\begin{array}{c}\text { Result/ } \\
\text { Uncertainty }\end{array}$ \\
\hline K-EE-620 & 02XP-01-A & U234 & $3.55 \mathrm{E}-04$ & $1.44 \mathrm{E}-04$ & 3.67E-05 & 2.5 \\
\hline K-EE-620 & 02XP-01-A & U238 & $2.94 E-04$ & $1.02 E-04$ & 4.85E-05 & 2.9 \\
\hline K-EE-621 & 02XP-02-A & U234 & $3.16 \mathrm{E}-04$ & 1.35E-04 & $3.73 E-05$ & 2.3 \\
\hline K-EE-621 & $02 X P-02-A$ & U238 & 2.98E-04 & 9.83E-05 & 3.46E-05 & 3.0 \\
\hline K-EE-622 & 02XP-03-A & U234 & 5.01E-04 & 1.62E-04 & 3.68E-05 & 3.1 \\
\hline K-EE-622 & 02XP-03-A & U238 & 5.39E-04 & $1.39 E-04$ & 2.74E-05 & 3.9 \\
\hline K-EE-622 & 02XP-03-A & PU239/240 & 7.31E-05 & 3.31E-05 & 3.31E-05 & 2.2 \\
\hline K-EE-623 & 02XP-04-A & U234 & 7.52E-04 & $1.91 E-04$ & $3.85 E-05$ & 3.9 \\
\hline K-EE-623 & 02XP-04-A & U238 & $6.57 E-04$ & $1.56 \mathrm{E}-04$ & 3.52E-05 & 4.2 \\
\hline K-EE-624 & 02XP-05-A & U234 & 5.47E-04 & 1.69E-04 & 4.13E-05 & 3.2 \\
\hline K-EE-624 & 02XP-05-A & U238 & $3.81 E-04$ & $1.16 \mathrm{E}-04$ & 4.13E-05 & 3.3 \\
\hline K-EE-625 & 02XP-06-A & U234 & 4.39E-04 & $1.56 \mathrm{E}-04$ & 4.57E-05 & 2.8 \\
\hline K-EE-625 & 02XP-06-A & U238 & 4.37E-04 & $1.25 E-04$ & $3.45 E-05$ & 3.5 \\
\hline K-EE-626 & 02XP-07-A & U234 & 8.11E-04 & 2.04E-04 & 4.33E-05 & 4.0 \\
\hline K-EE-626 & 02XP-07-A & U238 & 7.09E-04 & $1.70 E-04$ & 5.47E-05 & 4.2 \\
\hline K-EE-627 & 02XP-08-A & U234 & $6.48 \mathrm{E}-04$ & 1.77E-04 & 4.29E-05 & 3.7 \\
\hline K-EE-627 & $02 X P-08-A$ & U238 & $6.71 E-04$ & $1.57 E-04$ & $3.75 E-05$ & 4.3 \\
\hline K-EE-628 & $02 \times P-09-A$ & U234 & $3.86 E-04$ & $1.48 E-04$ & 4.34E-05 & 2.6 \\
\hline K-EE-628 & 02XP-09-A & U238 & $3.60 \mathrm{E}-04$ & 1.12E-04 & $3.69 E-05$ & 3.2 \\
\hline K-EE-638 & 02XP-19-A & U234 & 7.00E-04 & 2.05E-04 & $5.22 E-05$ & 3.4 \\
\hline K-EE-638 & $02 X P-19-A$ & U238 & 6.81E-04 & 1.75E-04 & $5.22 \mathrm{E}-05$ & 3.9 \\
\hline K-EE-638 & $02 X P-19-A$ & PU238 & 5.75E-03 & 6.27E-04 & 3.73E-05 & 9.2 \\
\hline K-EE-638 & 02XP-19-A & PU239/240 & $6.41 \mathrm{E}-03$ & $6.88 E-04$ & $1.47 E-05$ & 9.3 \\
\hline K-EE-638 & $02 X P-19-A$ & AM241 & 3.47E-03 & $3.13 E-04$ & 3.32E-05 & 11.1 \\
\hline
\end{tabular}




\begin{tabular}{|c|c|c|c|c|c|c|}
\hline $\begin{array}{l}\text { Customer } \\
\text { ID }\end{array}$ & Lab ID & Isotope & $\begin{array}{l}\text { Result } \\
\mathrm{Bq} / \mathrm{g}\end{array}$ & $\begin{array}{c}\text { Uncertainty } \\
\mathrm{Bq} / \mathrm{g}\end{array}$ & MDA & $\begin{array}{c}\text { Result/ } \\
\text { Uncertainty }\end{array}$ \\
\hline K-FF-629 & $02 X P-10-A$ & U234 & 2.79E-04 & 1.34E-04 & 3.99E-05 & 2.1 \\
\hline K-FF-629 & $02 \times P-10-A$ & U238 & 2.64E-04 & $9.68 \mathrm{E}-05$ & $3.64 \mathrm{E}-05$ & 2.7 \\
\hline K-GG-630 & 02XP-11-A & U234 & $4.85 E-04$ & 1.65E-04 & 4.48E-05 & 2.9 \\
\hline K-GG-630 & 02XP-11-A & U238 & $4.75 \mathrm{E}-04$ & 1.34E-04 & $4.48 \mathrm{E}-05$ & 3.5 \\
\hline K-GG-631 & $02 \times P-12-A$ & บ234 & $2.50 \mathrm{E}-04$ & 1.24E-04 & 3.17E-05 & 2.0 \\
\hline K-GG-631 & $02 X P-12-A$ & U238 & $3.41 E-04$ & $1.02 E-04$ & $2.90 \mathrm{E}-05$ & 3.3 \\
\hline K-GG-633 & $02 X P-14-A$ & U234 & 3.30E-04 & $1.41 \mathrm{E}-04$ & 4.32E-05 & 2.3 \\
\hline K-GG-633 & $02 X P-14-A$ & U238 & $3.60 \mathrm{E}-04$ & $1.11 \mathrm{E}-04$ & 3.26E-05 & 3.2 \\
\hline K-GG-634 & $02 X P-15-A$ & U238 & $2.50 \mathrm{E}-04$ & 9.69E-05 & 5.15E-05 & 2.6 \\
\hline $\mathrm{K}-\mathrm{HH}-635$ & 02XP-16-A & U234 & $8.12 E-04$ & $1.94 \mathrm{E}-04$ & 3.83E-05 & 4.2 \\
\hline $\mathrm{K}-\mathrm{HH}-635$ & $02 \times P-16-A$ & U238 & $5.80 \mathrm{E}-04$ & $1.41 \mathrm{E}-04$ & 3.56E-05 & 4.1 \\
\hline $\mathrm{K}-\mathrm{HH}-636$ & $02 X P-17-A$ & U234 & 4.82E-04 & $1.60 \mathrm{E}-04$ & 4.36E-05 & 3.0 \\
\hline $\mathrm{K}-\mathrm{HH}-636$ & $02 X P-17-A$ & U238 & $5.25 E-04$ & 1.38E-04 & 3.71E-05 & 3.8 \\
\hline $\mathrm{K}-\mathrm{HH}-636$ & 02XP-17-A & PU239/240 & 6.32E-05 & 3.02E-05 & 2.56E-05 & 2.1 \\
\hline $\mathrm{K}-\mathrm{HH}-637$ & 02XP-18-A & U234 & $4.02 \mathrm{E}-04$ & 1.51E-04 & 4.15E-09 & 2.7 \\
\hline $\mathrm{K}-\mathrm{HH}-637$ & $02 X P-18-A$ & U238 & $5.23 E-04$ & 1.38E-04 & 3.79E-05 & 3.8 \\
\hline $\mathrm{K}-\mathrm{HH}-639$ & 02XP-20-A & U234 & 8.77E-04 & 2.34E-04 & 5.21E-05 & 3.7 \\
\hline K-HH-639 & 02XP-20-A & U238 & 6.55E-04 & $1.72 E-04$ & 5.21E-05 & 3.8 \\
\hline $\mathrm{K}-\mathrm{HH}-639$ & 02XP-20-A & PU238 & $6.78 \mathrm{E}-03$ & 8.42E-04 & 1.27E-04 & 8.1 \\
\hline $\mathrm{K}-\mathrm{HH}-639$ & 02XP-20-A & PU239/240 & 3.98E-03 & $5.44 \mathrm{E}-04$ & 1.14E-04 & 7.3 \\
\hline $\mathrm{K}-\mathrm{HH}-639$ & $02 X P-20-A$ & AM241 & $4.78 \mathrm{E}-03$ & 5.03E-04 & $1.10 E-04$ & 9.5 \\
\hline
\end{tabular}

All known sources of uncertainty are included in the uncertainty term. There may be unknown sources of uncertainty that are not accounted for. If the result/uncertainty ratio is more than 3, we have a degree of confidence that the result is positive (i.e. the result is statistically different than zero). A result with the result/uncertainty ratio between 2 and 3 is the first indication that an isotope may be present and further investigation may be warranted.

As with any good science no single data point is used in important decisions (results need to be reproducible). 
RADIOANALYTICAL ANALYSES DATA PACKAGE

Project Title: $\quad$ Amchitka Island Alpha Analysis Report (Batch 16)

Lab Name:

RTC

Case No: NA

Report No.:

AmchitBatchF16

Method Type: A/B

Approved SAP No.: NA

SDG No: $\quad$ S-II-640

\section{SAMPLE NUMBERS}

Customer

Sample ID

\begin{tabular}{|c|}
\hline S-II-640 \\
\hline S-II-641 \\
\hline S-11-642 \\
\hline S-II-643 \\
\hline S-II-644 \\
\hline S-1I-645 \\
\hline S-11-646 \\
\hline S-11-647 \\
\hline S-11-648 \\
\hline S-S-649 \\
\hline S-S-651 \\
\hline S-S-652 \\
\hline S-S-653 \\
\hline S-S-654 \\
\hline S-S-655 \\
\hline S-S-656 \\
\hline S-S-657 \\
\hline S-S-658 \\
\hline S-S-659 \\
\hline
\end{tabular}

Lab Sample

ID

02YX-01-A

$02 Y X-02-A$

02YX-03-A

02YX-04-A

02YX-05-A

02YX-06-A

02YX-07-A

02YX-08-A

$02 Y X-09-A$

$02 Y X-10-A$

$02 Y X-12-A$

$02 Y X-13-A$

$02 Y X-14-A$

$02 Y X-15-A$

02YX-16-A

$02 Y X-17-A$

$02 Y X-18-A$

$02 Y X-19-A$

$02 Y X-20-A$

Comments:

Release of the data contained in this data package has been authorized by the laboratory manager or the manager's designee, as verified by the following signature:

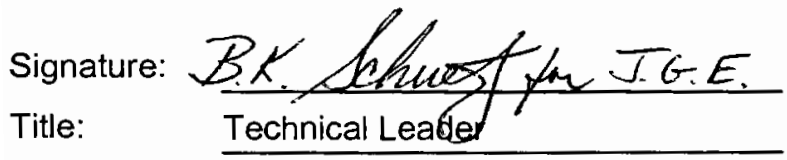

Name: J.G. Eisenmenger

Date: $\quad 11 / 22 / 2005$ 


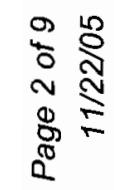

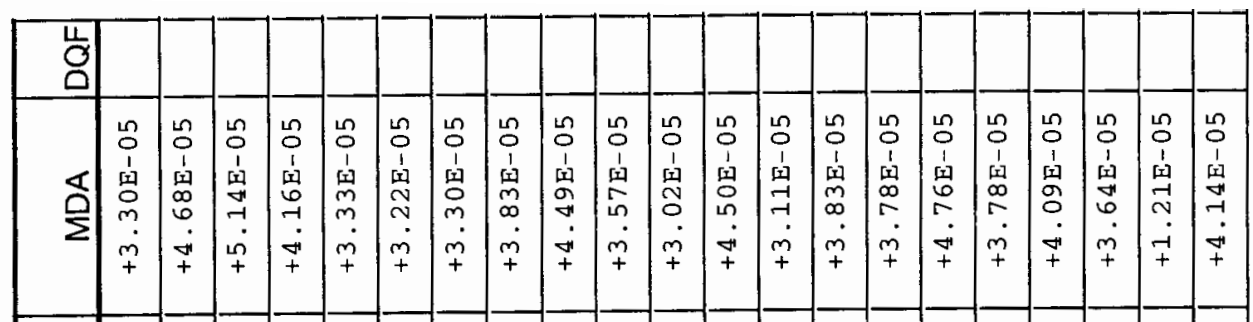

递

吾

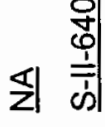

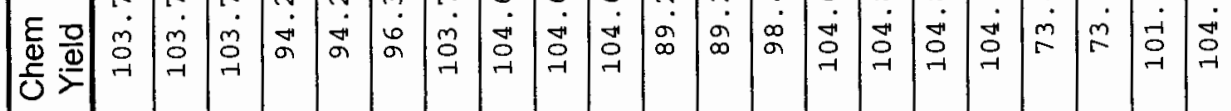

造

हूं

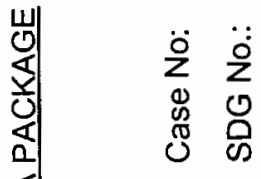

이의

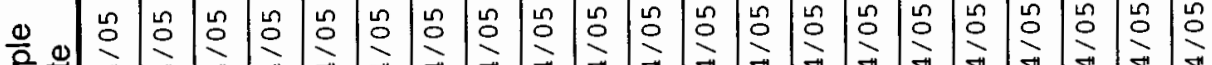

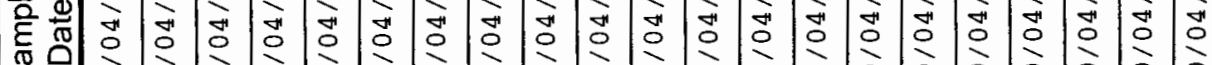
ळ

赵空

:

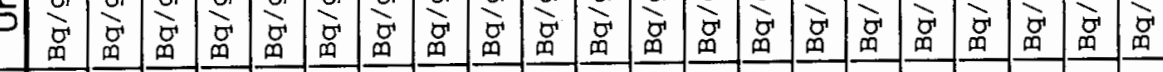

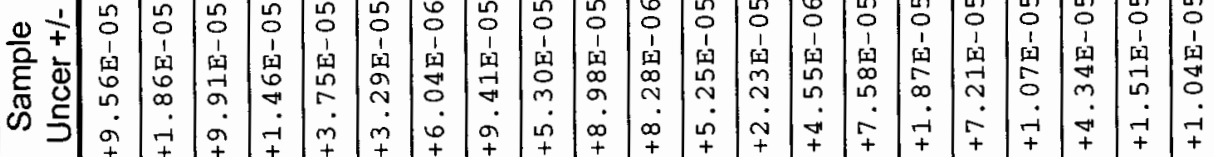

ఎ

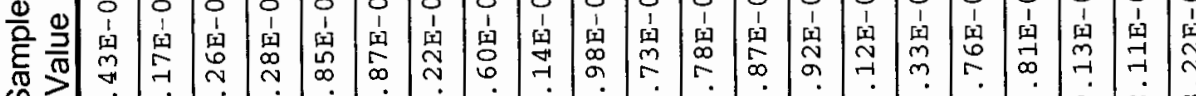

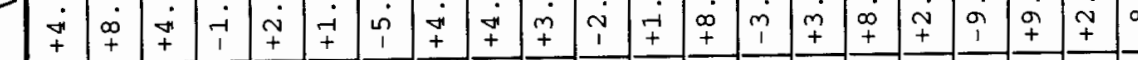

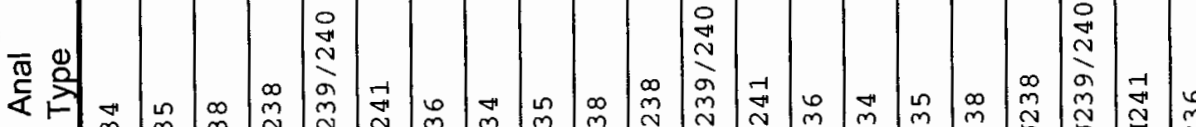

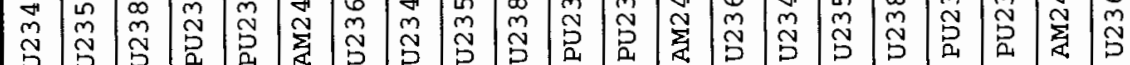

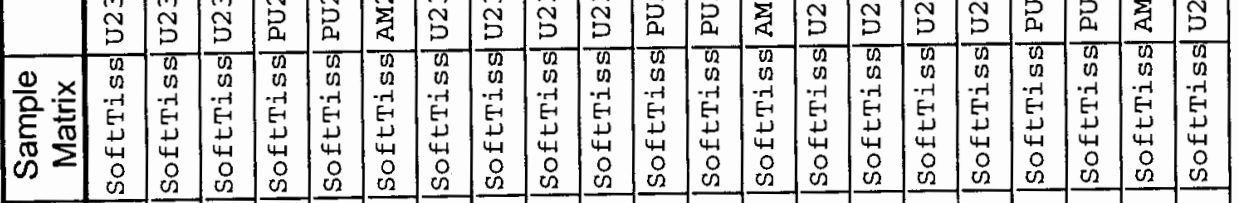

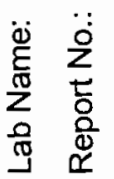

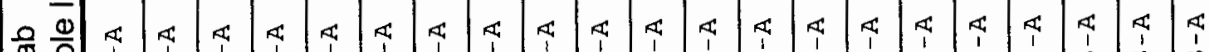

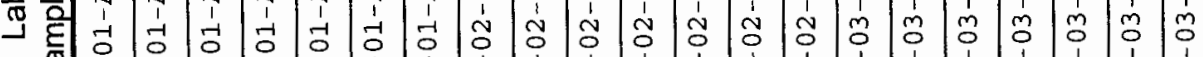

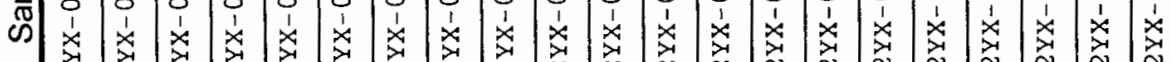

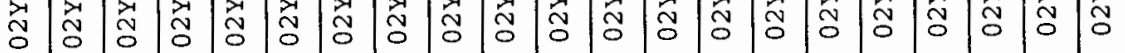


0
0
0
0
0
0
0
0
0

\begin{tabular}{|c|c|c|c|c|c|c|c|c|c|c|c|c|c|c|c|c|c|c|c|c|c|}
\hline 㝴 & $\begin{array}{l}2 \\
0 \\
1 \\
1 \\
1 \\
0 \\
0 \\
0 \\
\dot{0} \\
+\end{array}$ & $\begin{array}{l}n \\
0 \\
0 \\
1 \\
\text { q⿴囗十 } \\
0 \\
m \\
0 \\
+ \\
+\end{array}$ & $\begin{array}{l}n \\
0 \\
0 \\
1 \\
\text { q⿴囗十 } \\
0 \\
0 \\
0 \\
7 \\
+1\end{array}$ & $\begin{array}{l}n \\
0 \\
0 \\
1 \\
19 \\
0 \\
0 \\
0 \\
+1 \\
+\end{array}$ & $\begin{array}{l}n \\
0 \\
1 \\
1 \\
0 \\
71 \\
0 \\
+ \\
+\end{array}$ & $\begin{array}{l}n \\
0 \\
1 \\
\varphi \\
0 \\
0 \\
0 \\
0 \\
+ \\
+\end{array}$ & 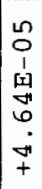 & $\begin{array}{l}n \\
0 \\
0 \\
1 \\
m \\
m \\
? \\
\dot{m} \\
+ \\
+\end{array}$ & 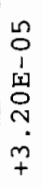 & 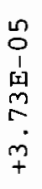 & $\begin{array}{l}n \\
0 \\
1 \\
1 \\
0 \\
0 \\
0 \\
\dot{m} \\
+ \\
+\end{array}$ & 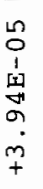 & $\begin{array}{l}n \\
0 \\
1 \\
1 \\
0 \\
0 \\
n \\
\dot{T} \\
+ \\
+\end{array}$ & 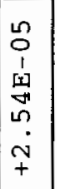 & $\begin{array}{l}n \\
0 \\
1 \\
1 \\
0 \\
0 \\
0 \\
+ \\
+ \\
\end{array}$ & $\begin{array}{l}0 \\
0 \\
0 \\
1 \\
1 \\
-1 \\
m \\
m \\
-1 \\
+ \\
+\end{array}$ & $\begin{array}{l}n \\
0 \\
0 \\
1 \\
1 \\
\\
0 \\
\dot{\sim} \\
+ \\
+\end{array}$ & 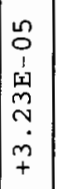 & $\begin{array}{l}n \\
0 \\
0 \\
1 \\
w \\
n \\
n \\
n \\
w \\
+\end{array}$ & $\begin{array}{l}n \\
0 \\
1 \\
1 \\
m \\
m \\
m \\
m \\
\sim \\
+ \\
+\end{array}$ & $\mid \begin{array}{l}n \\
0 \\
1 \\
1 \\
01 \\
+1 \\
0 \\
-1 \\
+1 \\
+\end{array}$ \\
\hline 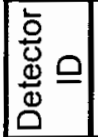 & $\stackrel{2}{\circ}$ & 10 & $\stackrel{2}{\circ}$ & {$\left[\begin{array}{l}n \\
-1\end{array}\right.$} & $\stackrel{n}{\rightarrow}$ & $\underset{\nearrow}{\mp}$ & | & $\because \%$ & ${ }^{\circ}$ & $\stackrel{\circ}{\circ}$ & $\stackrel{\stackrel{n}{\rightarrow}}{\rightarrow-1}$ & $\stackrel{\stackrel{\sim}{\rightarrow}}{\rightarrow}$ & $\rightarrow$ & $\%$ & 5 & 5 & 5 & $\stackrel{\leftrightarrow}{\sim}$ & $\underset{r-1}{0}$ & $\underset{-}{\vec{H}}$ & ó \\
\hline 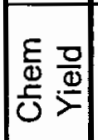 & $\begin{array}{c}-1 \\
- \\
- \\
-1\end{array}$ & - & $\begin{array}{l}\vec{r} \\
\overrightarrow{-} \\
- \\
-1\end{array}$ & a & नुं & $\begin{array}{l}\tilde{n} \\
\infty \\
\sigma \\
\sigma\end{array}$ & न्. & $\begin{array}{l}\overline{6} \\
0 \\
\exists \\
-1\end{array}$ & $\begin{array}{l}0 \\
\dot{0} \\
7 \\
-1\end{array}$ & $\begin{array}{l}\overrightarrow{0} \\
\dot{\sigma} \\
\vec{\sigma}\end{array}$ & r. & 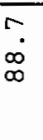 & \begin{tabular}{l} 
\\
\hdashline \\
0 \\
0 \\
-1
\end{tabular} & 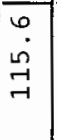 & $\begin{array}{l}r \\
0 \\
0 \\
-1\end{array}$ & $\begin{array}{l}0 \\
0 \\
0 \\
0 \\
-1\end{array}$ & $\begin{array}{l} \\
\dot{0} \\
0 \\
0 \\
-1\end{array}$ & \begin{tabular}{|l|}
-1 \\
$\dot{0}$ \\
$\infty$ \\
$\infty$
\end{tabular} & $\begin{array}{l}7 \\
\dot{b} \\
\infty \\
\infty\end{array}$ & $\begin{array}{l}\overline{0} \\
\dot{0} \\
0\end{array}$ & r. \\
\hline 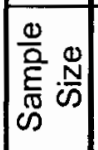 & 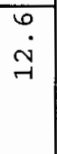 & $\overline{0}$ & 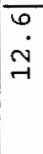 & $\begin{array}{l}\overline{0} \\
\dot{\sim} \\
-1\end{array}$ & \begin{tabular}{l}
$\overline{0}$ \\
$\dot{w}$ \\
\multirow{\gamma}{*}{}
\end{tabular} & $\begin{array}{l}\overline{0} \\
\dot{\sim} \\
7\end{array}$ & $\begin{array}{l}\overline{0} \\
\dot{y} \\
-1\end{array}$ & In & $\begin{array}{l}5 \\
\sim\end{array}$ & $\stackrel{n}{\sim}$ & $\stackrel{\Omega}{\sim}$ & $\begin{array}{l}n \\
\sim\end{array}$ & $\begin{array}{l}n \\
n \\
\rightarrow 1\end{array}$ & \begin{tabular}{|l|}
\multirow{2}{n}{} \\
$\rightarrow$
\end{tabular} & $\begin{array}{ll}n \\
-1\end{array}$ & $\begin{array}{l}n \\
\rightarrow\end{array}$ & $\begin{array}{l}n \\
\rightarrow\end{array}$ & 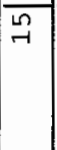 & ח & na & 点 \\
\hline
\end{tabular}

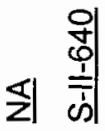

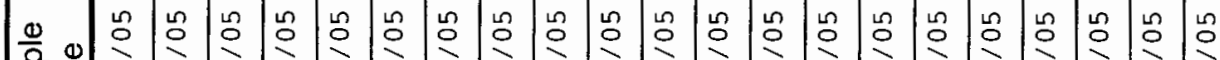

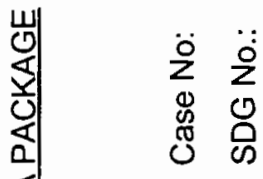

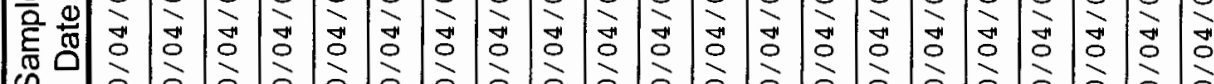

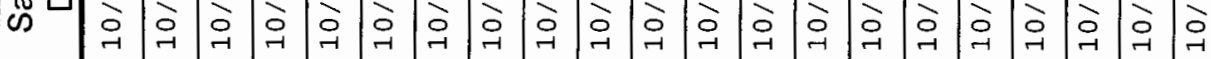

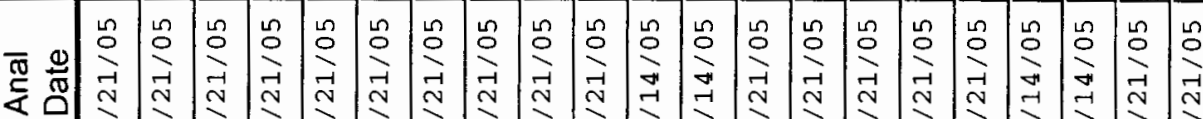

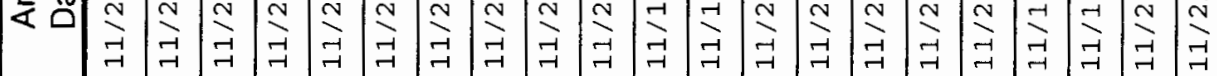

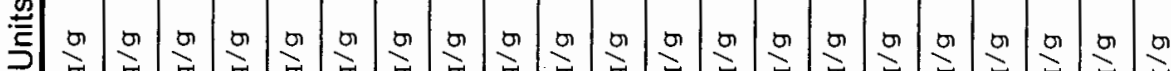

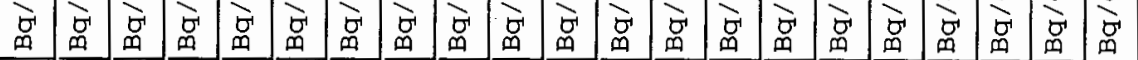

凹卉

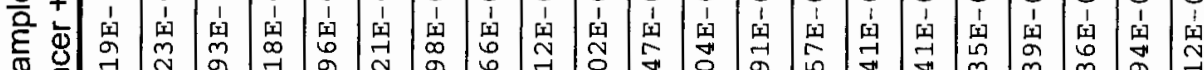

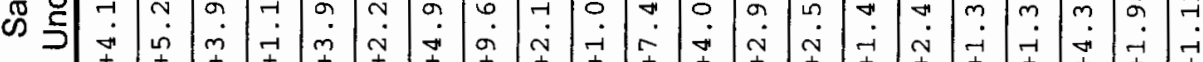
※ ह

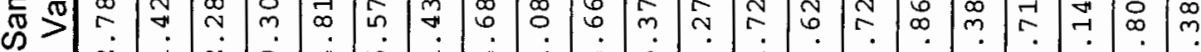

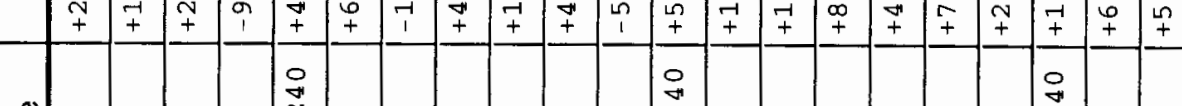
这 齐

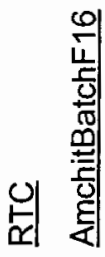
$m$ m

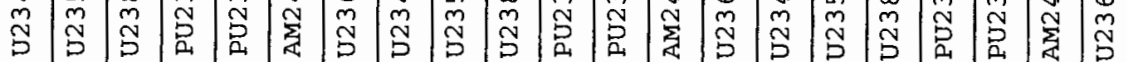

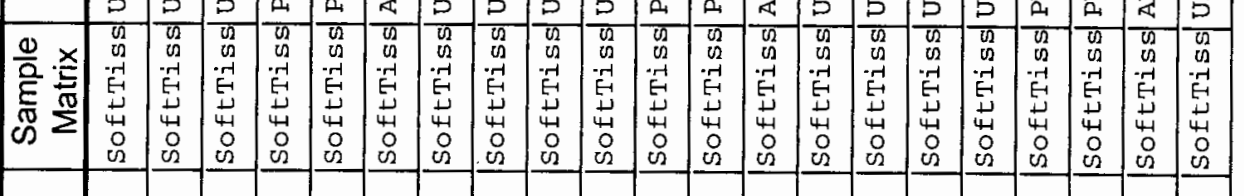

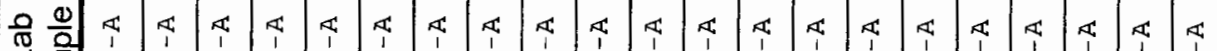

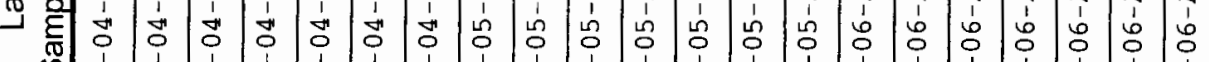

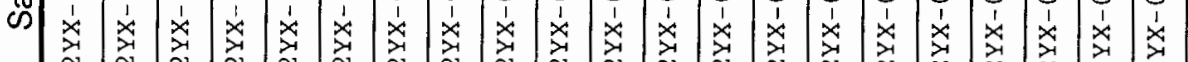
茫

으

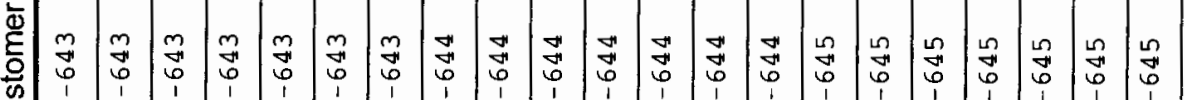

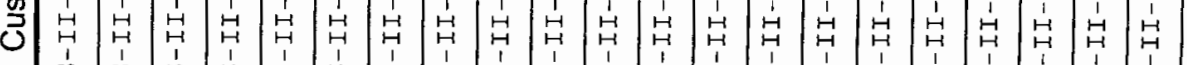




\begin{tabular}{|c|c|c|c|c|c|c|c|c|c|c|c|c|c|c|c|c|c|c|c|c|c|}
\hline $\begin{array}{l} \\
\\
\end{array}$ & & & & & & & & & & & & & & & & & & & & & \\
\hline 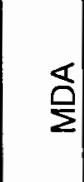 & $\begin{array}{l}n \\
0 \\
1 \\
1 \\
\text { I } \\
\stackrel{7}{7} \\
+ \\
+ \\
+\end{array}$ & $\begin{array}{l}n \\
0 \\
1 \\
x \\
1 \\
N \\
\vdots \\
j \\
+\end{array}$ & $\begin{array}{l}20 \\
0 \\
1 \\
0 \\
0 \\
0 \\
\dot{3} \\
+ \\
+\end{array}$ & $\begin{array}{l}n \\
0 \\
1 \\
1 \\
0 \\
0 \\
+ \\
+ \\
m \\
+ \\
+\end{array}$ & $\begin{array}{l}n \\
0 \\
1 \\
w \\
a \\
\sigma \\
o \\
m \\
+ \\
+\end{array}$ & $\begin{array}{l}n \\
0 \\
i \\
\dot{1} \\
0 \\
0 \\
\dot{n} \\
\dot{m} \\
+\end{array}$ & $\begin{array}{l}n \\
0 \\
1 \\
1 \\
0 \\
0 \\
0 \\
+1 \\
-1 \\
+\end{array}$ & $\begin{array}{l}n \\
0 \\
1 \\
1 \\
0 \\
0 \\
+ \\
+ \\
+ \\
+\end{array}$ & $\begin{array}{l}n \\
0 \\
1 \\
\text { y } \\
9 \\
+ \\
\vdots \\
0 \\
+\end{array}$ & $\begin{array}{l}n \\
0 \\
1 \\
1 \\
0 \\
0 \\
\square \\
\vdots \\
0 \\
+\end{array}$ & $\begin{array}{l}n \\
0 \\
1 \\
1 \\
\hat{n} \\
\tilde{n} \\
\tilde{n} \\
+ \\
+\end{array}$ & 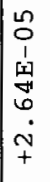 & $\begin{array}{l}n \\
0 \\
1 \\
1 \\
1 \\
0 \\
0 \\
0 \\
+ \\
+\end{array}$ & $\begin{array}{l}\text { n } \\
0 \\
1 \\
\text { th } \\
-1 \\
-1 \\
+1 \\
+\end{array}$ & $\begin{array}{l}n \\
0 \\
0 \\
1 \\
1 \\
y \\
+1 \\
\dot{m} \\
+ \\
+\end{array}$ & $\mid \begin{array}{l}n \\
0 \\
1 \\
1 \\
\text { ⿷1 } \\
0 \\
n \\
i \\
+ \\
+\end{array}$ & 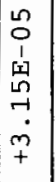 & $\begin{array}{l}n \\
0 \\
1 \\
1 \\
0 \\
0 \\
0 \\
n \\
+\end{array}$ & 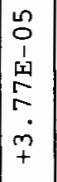 & $\begin{array}{l}n \\
0 \\
1 \\
1 \\
0 \\
\infty \\
\infty \\
\infty \\
0 \\
+\end{array}$ & 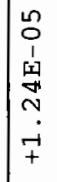 \\
\hline פ & 冓 & ${ }^{\infty}$ & $\left.\right|_{0} ^{\infty}$ & Ig & 10 & $\stackrel{\sim}{\sim}$ & $0^{\infty}$ & $\overrightarrow{0}$ & $\overrightarrow{0}$ & |t & 욱 & $\stackrel{\circ}{r}$ & 帛 & $\sigma$ & 5 & 5 & 10 & : & \% & $\stackrel{\infty}{\circ}$ & ó \\
\hline 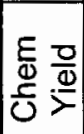 & \begin{tabular}{|l}
$n$ \\
0 \\
0 \\
0 \\
$r$
\end{tabular} & ? & $\begin{array}{l}n \\
\dot{D} \\
0 \\
\end{array}$ & $\begin{array}{l}m \\
\dot{m} \\
\dot{\infty}\end{array}$ & $\begin{array}{l}m \\
\dot{m} \\
\dot{0}\end{array}$ & m. & $\begin{array}{l}n \\
\overrightarrow{0} \\
0\end{array}$ & ف. & $\begin{array}{l}0 \\
\dot{n} \\
-1 \\
-1\end{array}$ & $\begin{array}{l}0 \\
0 \\
\sim \\
-1\end{array}$ & ? & $\begin{array}{l}9 \\
1 \\
\infty \\
\infty\end{array}$ & 它 & 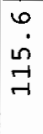 & r. & $\begin{array}{l}-1 \\
\dot{m} \\
0 \\
-\end{array}$ & $\begin{array}{l}\vec{y} \\
\dot{m} \\
\stackrel{-}{r}\end{array}$ & $\begin{array}{l}\infty \\
\dot{\sigma} \\
0 \\
0 \\
-1\end{array}$ & 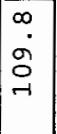 & $\begin{array}{l}\ddot{ } \\
- \\
ت \\
ت\end{array}$ & 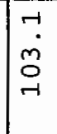 \\
\hline
\end{tabular}

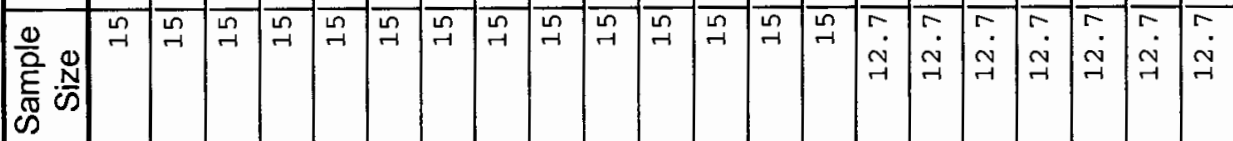
$\sum \frac{\stackrel{9}{0}}{=}$

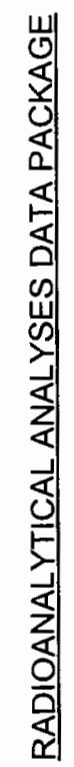

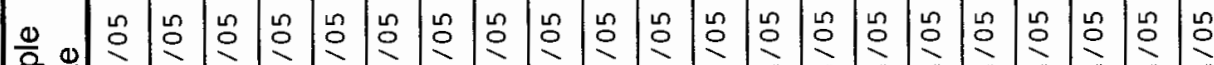

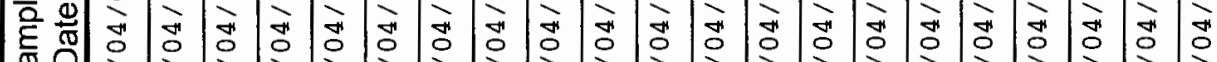
๙

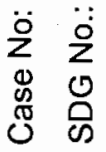

可

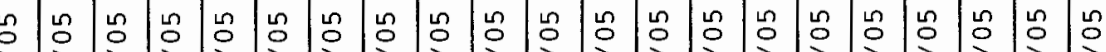

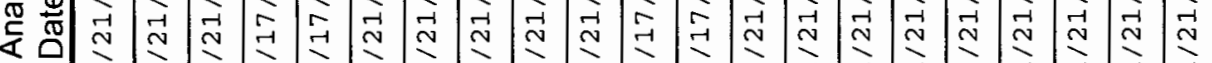

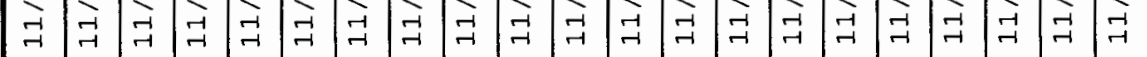

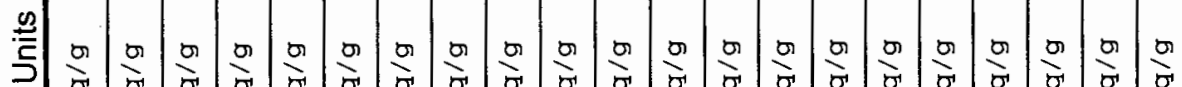

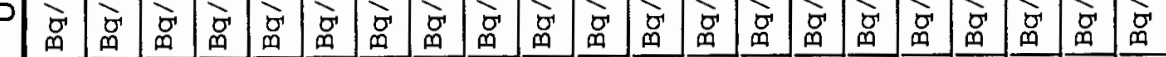

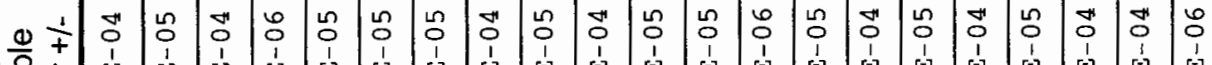

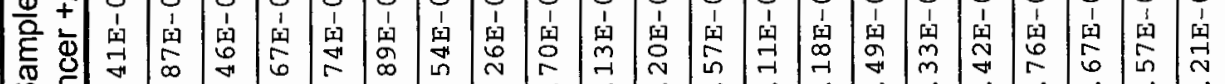

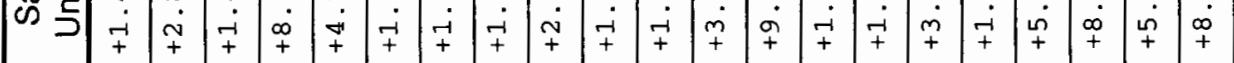

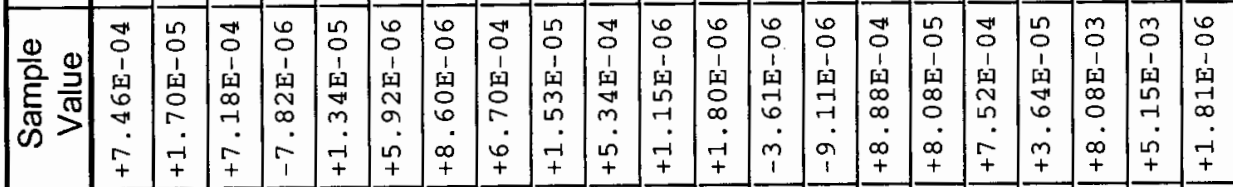
ত \&

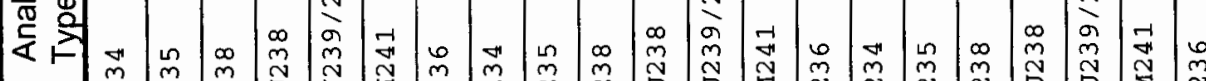

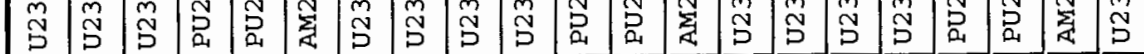

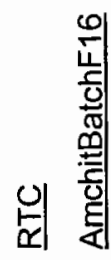

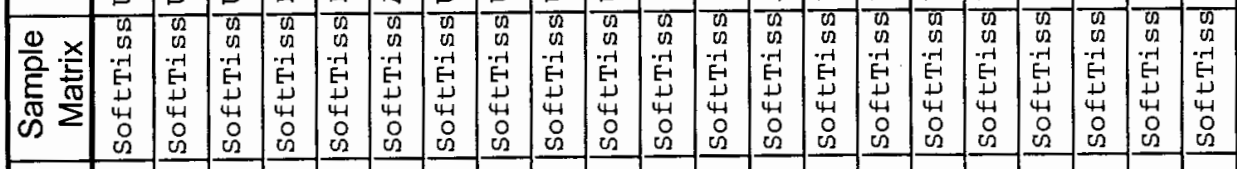
으

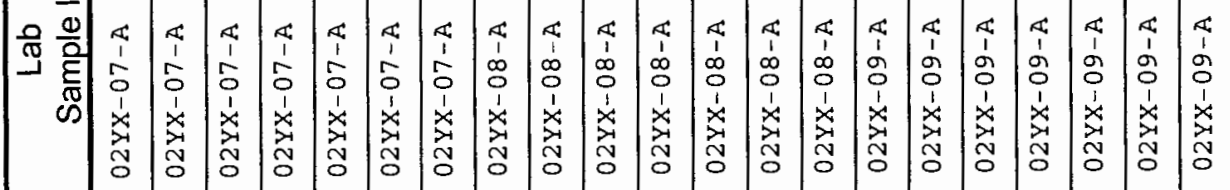

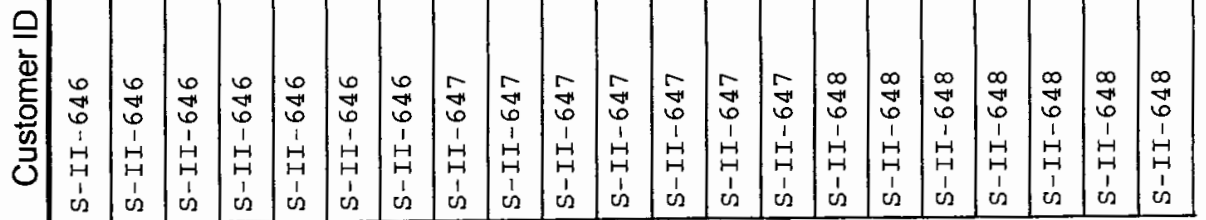


0
0
0
0
0
0
8
0
0

\begin{tabular}{|c|c|c|c|c|c|c|c|c|c|c|c|c|c|c|c|c|c|c|c|c|c|}
\hline $\begin{array}{l}1 \\
0 \\
0\end{array}$ & & & & & & & & & & & & & & & & & & & & & \\
\hline$\stackrel{\rho}{\Sigma}$ & $\begin{array}{l}\stackrel{n}{0} \\
1 \\
1 \\
\sigma \\
\sigma \\
r \\
\dot{m} \\
+\end{array}$ & $\begin{array}{l}n \\
0 \\
1 \\
1 \\
\mathbf{9} \\
0 \\
0 \\
0 \\
+ \\
+\end{array}$ & 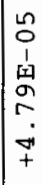 & $\begin{array}{l}n \\
0 \\
1 \\
1 \\
1 \\
5 \\
m \\
0 \\
+ \\
+\end{array}$ & 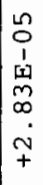 & $\begin{array}{l}\qquad n \\
0 \\
1 \\
\text { ch } \\
m \\
v \\
v \\
m \\
+\end{array}$ & $\begin{array}{l}n \\
0 \\
1 \\
1 \\
0 \\
0 \\
0 \\
m \\
+ \\
+\end{array}$ & $\begin{array}{l}n \\
0 \\
1 \\
1 \\
a \\
m \\
m \\
1 \\
+ \\
+\end{array}$ & 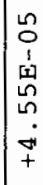 & 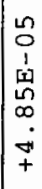 & 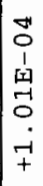 & $\begin{array}{l}n \\
0 \\
1 \\
1 \\
\vdots \\
m \\
? \\
0 \\
+ \\
+\end{array}$ & $\begin{array}{l}n \\
0 \\
1 \\
1 \\
1 \\
0 \\
0 \\
0 \\
-\dot{1} \\
+ \\
+\end{array}$ & 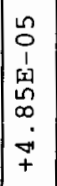 & $\begin{array}{l}n \\
0 \\
1 \\
1 \\
1 \\
\infty \\
\infty \\
m \\
m \\
+\end{array}$ & 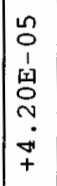 & \begin{tabular}{|l}
$n$ \\
0 \\
1 \\
$w$ \\
$y$ \\
$m$ \\
$m$ \\
$m$ \\
+ \\
+
\end{tabular} & $\begin{array}{l}n \\
0 \\
1 \\
y \\
n \\
n \\
\vdots \\
7 \\
+ \\
+\end{array}$ & $\begin{array}{l}n \\
0 \\
1 \\
1 \\
1 \\
0 \\
0 \\
0 \\
\dot{m} \\
+\end{array}$ & \begin{tabular}{l}
$n$ \\
0 \\
1 \\
1 \\
\multicolumn{1}{|c}{} \\
-1 \\
+1 \\
$\dot{n}$ \\
+
\end{tabular} & 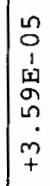 \\
\hline \begin{tabular}{|l|}
$\frac{0}{0}$ \\
$\frac{d}{0}$ \\
$\frac{d}{0}$ \\
0
\end{tabular} & $\tilde{O}$ & $\tilde{\delta}$ & SO & $\exists$ & 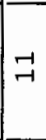 & \% & $\tilde{O}$ & |옹 & 告 & In & $\sum_{0}^{\infty}$ & $\stackrel{\infty}{\circ}$ & $\exists$ & In & on & 0 & o & $\approx$ & $\exists$ & 욱 & m \\
\hline$\frac{E}{d} \frac{ㅁ}{0}$ & $\begin{array}{l}m \\
-\vec{z} \\
\vec{r}\end{array}$ & $\begin{array}{c}m \\
\stackrel{-}{7} \\
\vec{r}\end{array}$ & $\begin{array}{l}m \\
\stackrel{-}{F} \\
F\end{array}$ & $\stackrel{v}{a}$ & $\frac{v}{a}$ & $\begin{array}{l} \\
\dot{0} \\
\sigma\end{array}$ & $\begin{array}{l}m \\
\stackrel{m}{-} \\
ت\end{array}$ & 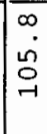 & $\begin{array}{l}\infty \\
\dot{n} \\
\dot{0} \\
-1\end{array}$ & $\begin{array}{l}\infty \\
\dot{\rho} \\
\text { مे } \\
r\end{array}$ & m. & m. & $\begin{aligned} r-1 \\
\therefore \\
\circ \\
-1\end{aligned}$ & 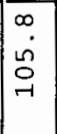 & $\begin{array}{ll}\infty \\
- \\
- \\
-\end{array}$ & $\begin{array}{l}\infty \\
- \\
- \\
ت \\
-\end{array}$ & $\begin{array}{l}\infty \\
\exists \\
\exists \\
\exists\end{array}$ & $\begin{array}{l} \\
\dot{n} \\
\infty \\
\infty\end{array}$ & \begin{tabular}{|l|}
$n$ \\
$\dot{\infty}$ \\
$\infty$
\end{tabular} & & $\begin{array}{l}\infty \\
- \\
- \\
-\end{array}$ \\
\hline 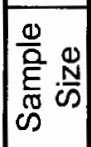 & $\stackrel{\stackrel{\sim}{\sim}}{\sim}$ & $\mid$ & 莴 & $\stackrel{n}{\sim}$ & 욕 & $\sqrt{-1}$ & 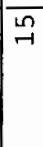 & $\begin{array}{l}n \\
\check{\tau} \\
\tau\end{array}$ & 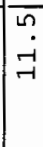 & $\vec{f}$ & न & 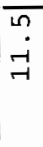 & $\begin{array}{l}n \\
- \\
-\end{array}$ & 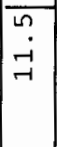 & $\sqrt[\pi]{7}$ & $\begin{array}{l}\tilde{r} \\
\sim\end{array}$ & $\stackrel{\Omega}{\sim}$ & $\begin{array}{l}\tilde{r} \\
-1\end{array}$ & ${ }_{n-1}^{n}$ & $\stackrel{5}{-1}$ & 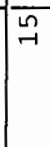 \\
\hline
\end{tabular}

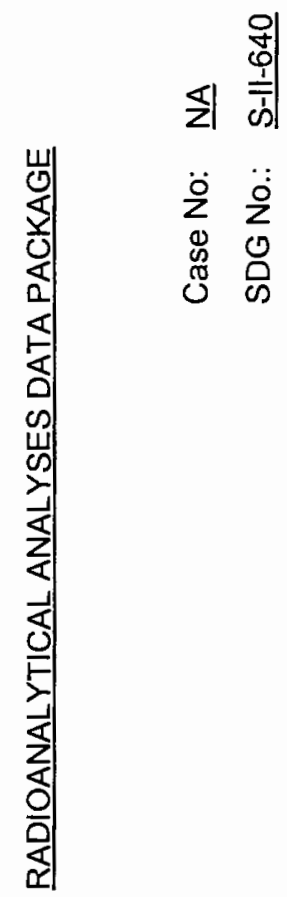

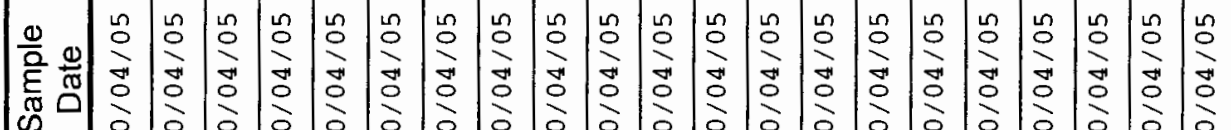
œ

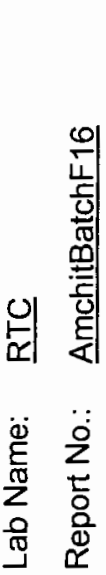

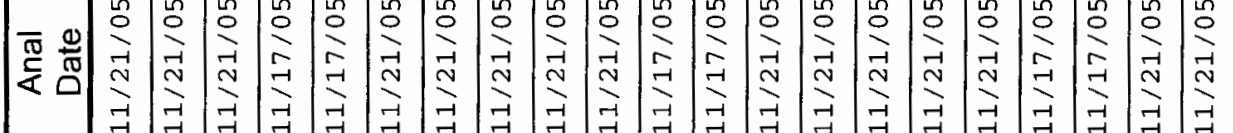

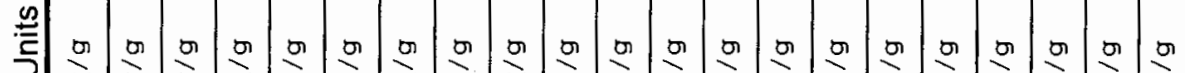

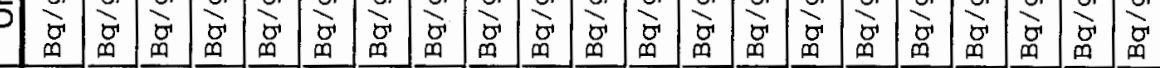

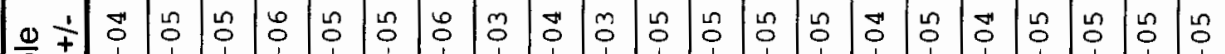

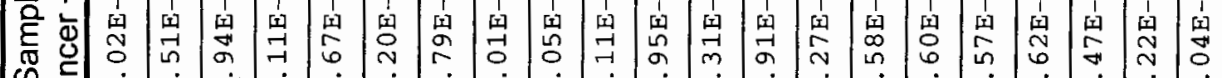

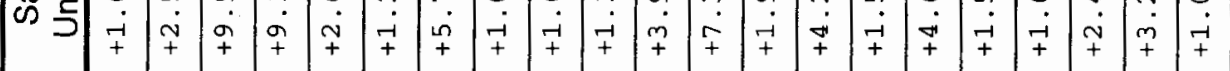
世

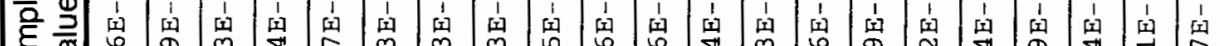

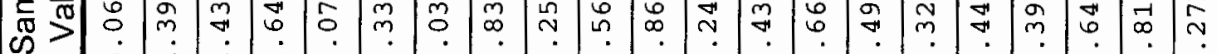

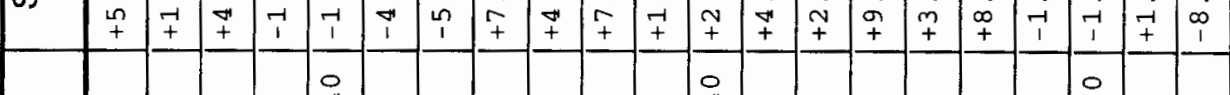

产龱

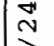

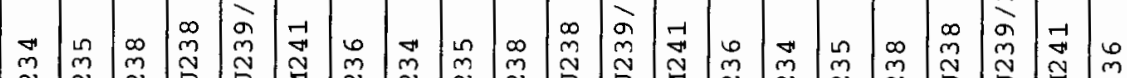

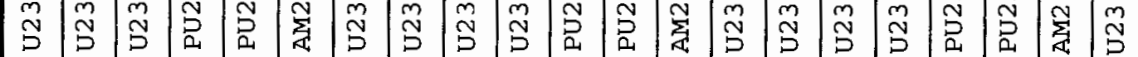

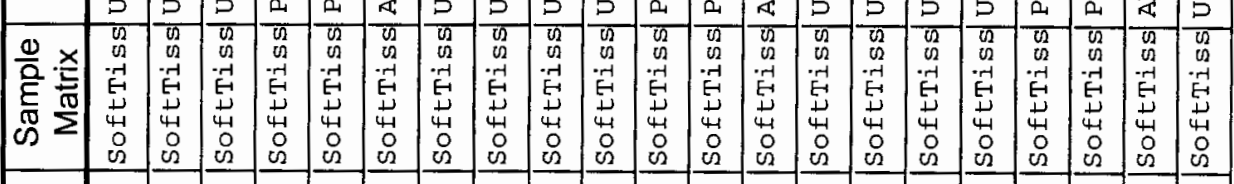
은

每

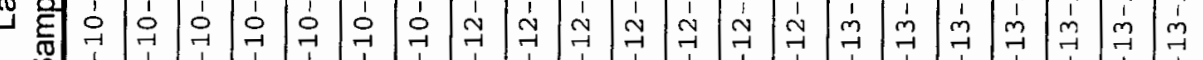

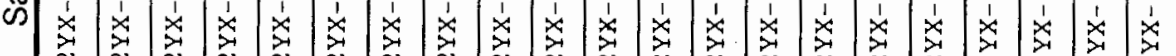
空

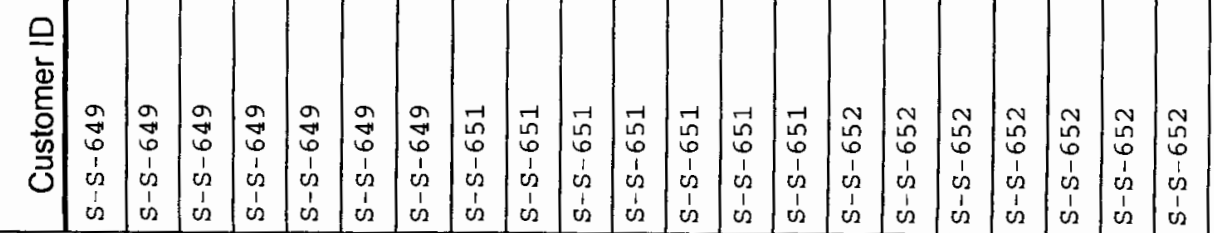




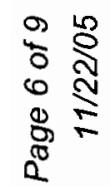

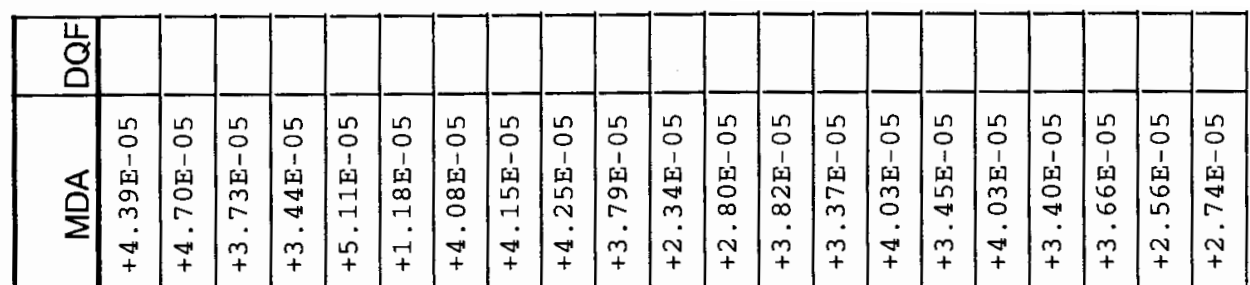

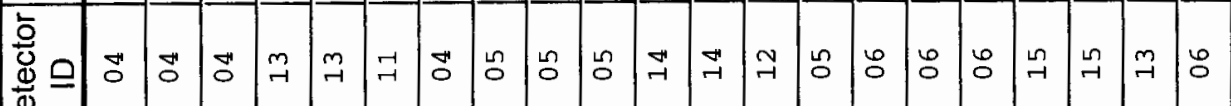
要

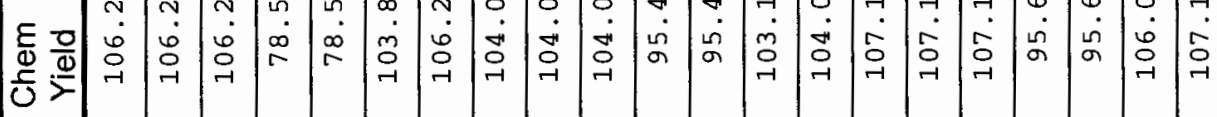

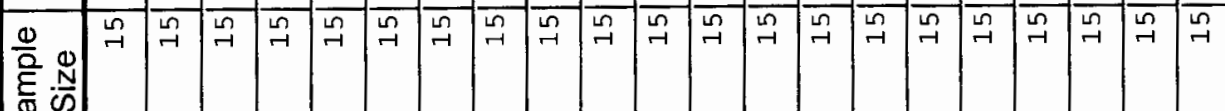

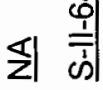

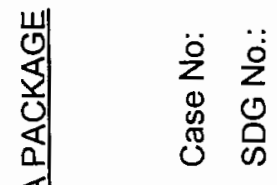

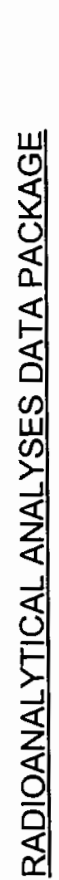

๗ํㅇㅇ

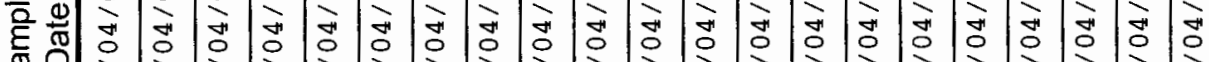
ळ ळ रू

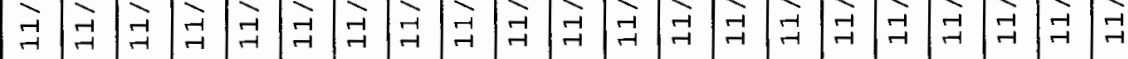

: a a a a a a a a a a a a a

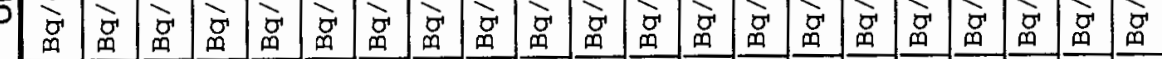

我

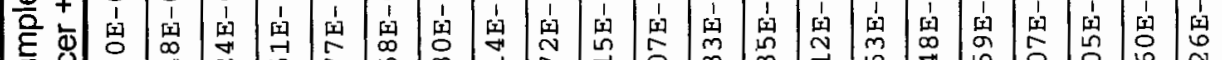
ळ

1

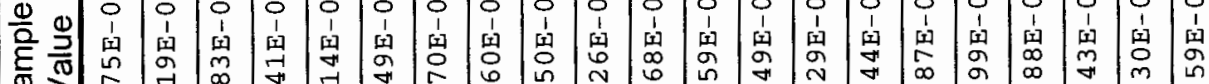
ळ $>$ ळ

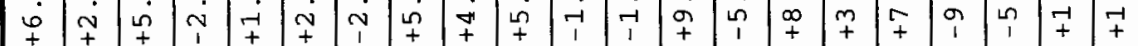

琎离

온

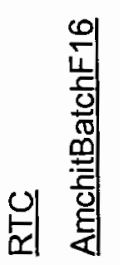

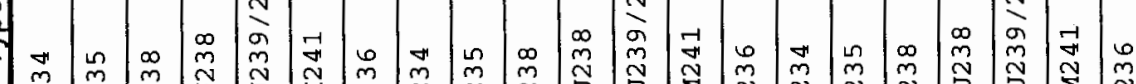

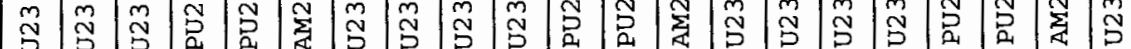

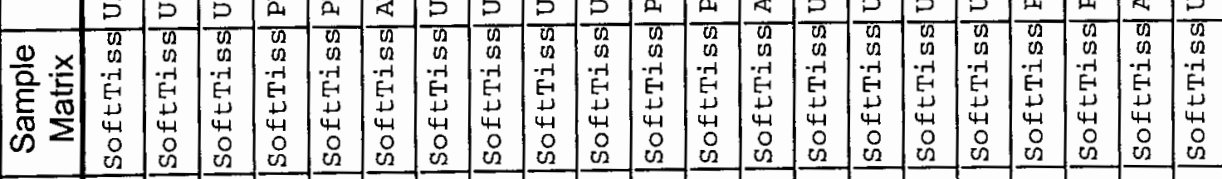

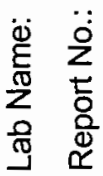

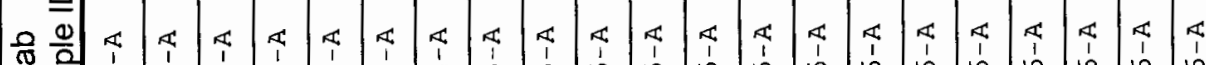

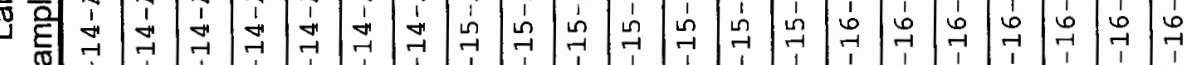

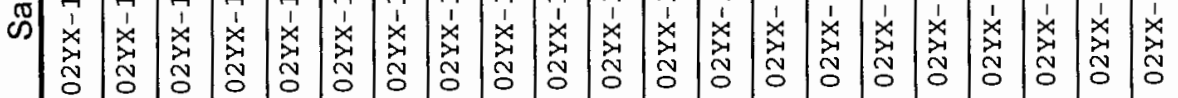

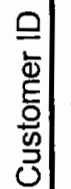

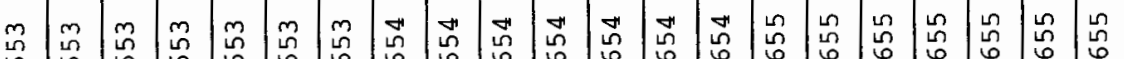




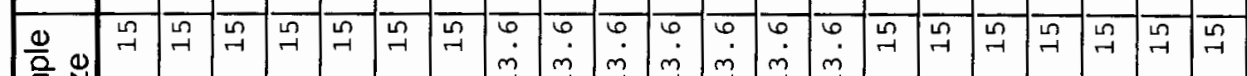
离

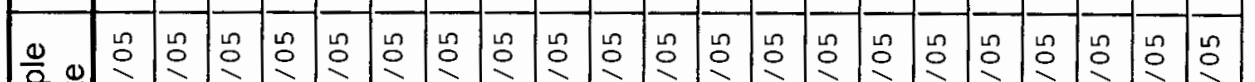

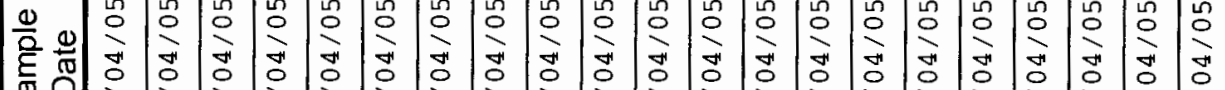

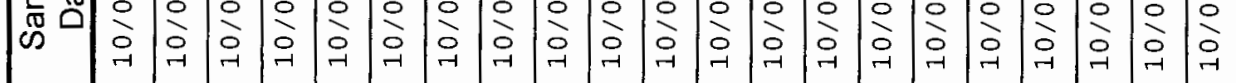

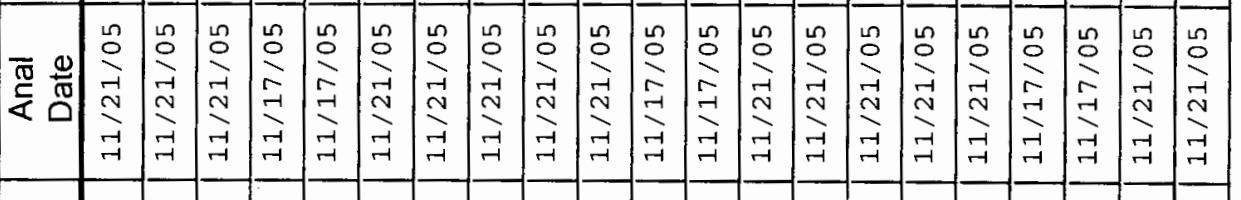

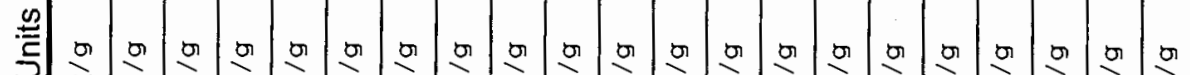

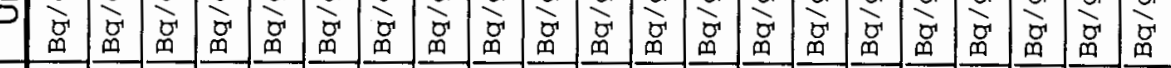
番

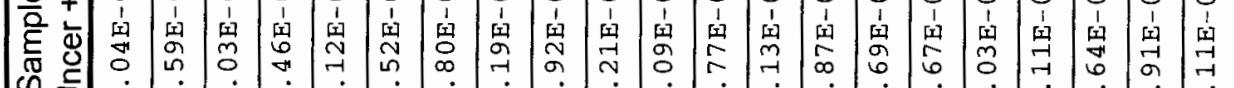

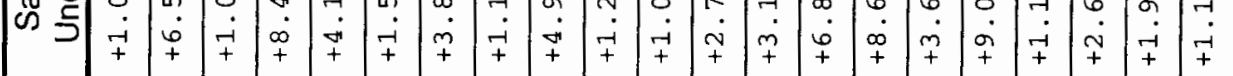

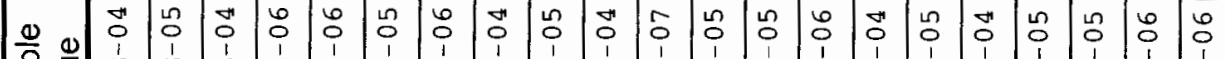

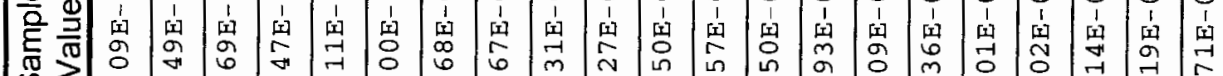

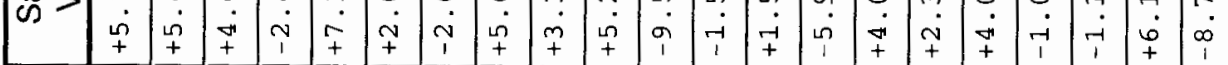

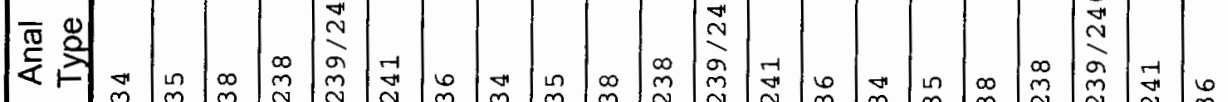

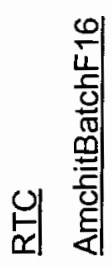

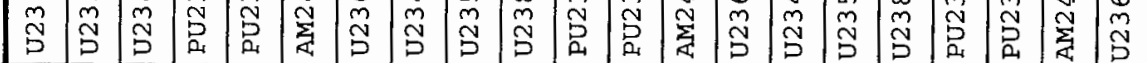

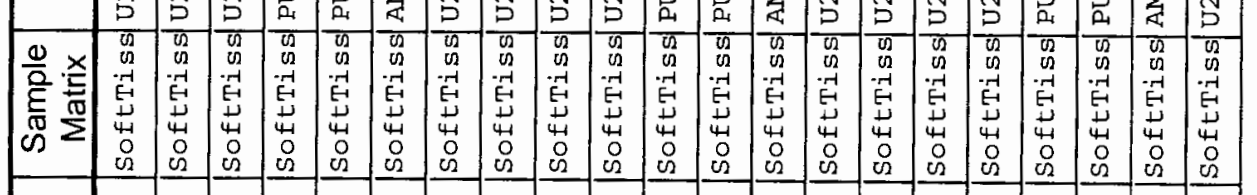

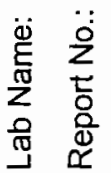

道

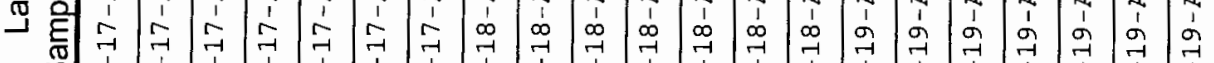

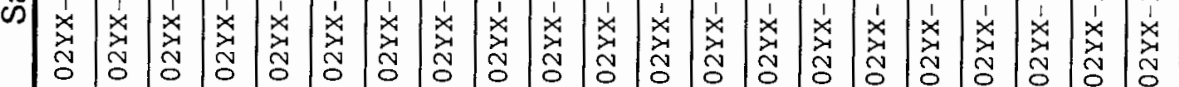

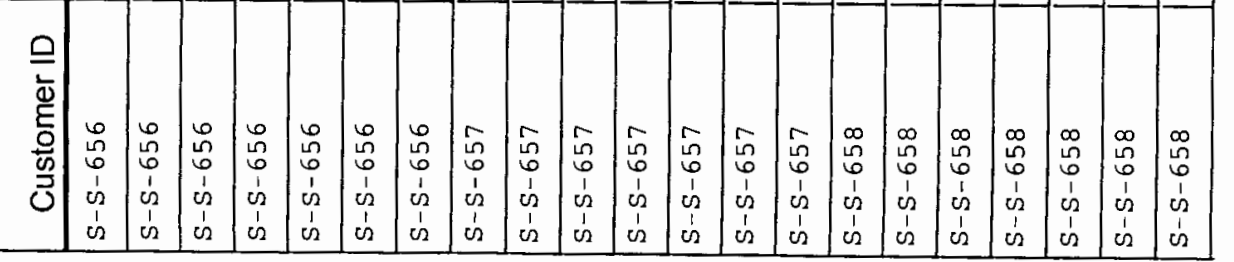




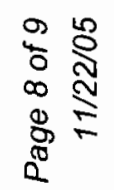

\begin{tabular}{|c|c|c|c|c|c|c|c|}
\hline \begin{tabular}{l|} 
岁 \\
\end{tabular} & & & & & & & \\
\hline 吉 & 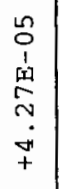 & $\begin{array}{l}n \\
0 \\
1 \\
1 \\
\text { In } \\
5 \\
j \\
+ \\
+\end{array}$ & $\begin{array}{l}n \\
0 \\
1 \\
1 \\
n \\
0 \\
0 \\
\dot{m} \\
+\end{array}$ & 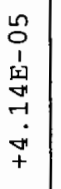 & $\begin{array}{c}n \\
0 \\
1 \\
1 \\
\infty \\
0 \\
0 \\
\dot{m} \\
+ \\
+\end{array}$ & 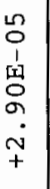 & $\begin{array}{l}n \\
0 \\
1 \\
1 \\
\mathbf{1} \\
5 \\
\vdots \\
\dot{m} \\
+\end{array}$ \\
\hline 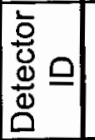 & $\overrightarrow{0}$ & $\overrightarrow{0}$ & $\overrightarrow{0}$ & 욱 & 욱 & 8 & $\sigma^{*}$ \\
\hline$\left|\begin{array}{ll}\varepsilon & 0 \\
\frac{D}{S} & \frac{0}{0} \\
& =\end{array}\right|$ & $\begin{array}{l}\vec{y} \\
\dot{0} \\
\circ \\
-\end{array}$ & 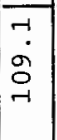 & $\begin{array}{l}-1 \\
\dot{\sigma} \\
\circ \\
-\end{array}$ & $\begin{array}{l}\overrightarrow{0} \\
\dot{n}\end{array}$ & $\begin{array}{l}0 \\
\dot{n} \\
r\end{array}$ & $\begin{array}{l} \\
\dot{S} \\
\dot{0} \\
-1\end{array}$ & $\begin{array}{l}\overrightarrow{1} \\
\dot{0} \\
0 \\
-1\end{array}$ \\
\hline 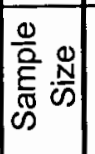 & $\stackrel{4}{\neg}$ & \begin{tabular}{|l|}
$\Omega$ \\
$\neg$
\end{tabular} & \begin{tabular}{|l|}
$n$ \\
-1
\end{tabular} & 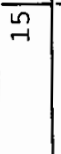 & $\cong$ & ñ & ח \\
\hline 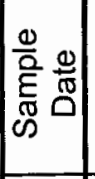 & $\begin{array}{c}n \\
0 \\
\vdots \\
0 \\
0 \\
0 \\
-1 \\
\end{array}$ & \begin{tabular}{|l|}
$n$ \\
0 \\
$o$ \\
0 \\
0 \\
0 \\
-1 \\
\end{tabular} & $\begin{array}{l}0 \\
0 \\
0 \\
0 \\
0 \\
0 \\
-1 \\
\end{array}$ & \begin{tabular}{|l}
0 \\
0 \\
0 \\
0 \\
0 \\
0 \\
0 \\
-1 \\
\end{tabular} & $\begin{array}{l}2 \\
0 \\
\vdots \\
0 \\
0 \\
0 \\
-1 \\
\end{array}$ & 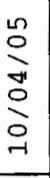 & $\begin{array}{l}n \\
0 \\
0 \\
0 \\
0 \\
0 \\
-1 \\
\end{array}$ \\
\hline$\left|\begin{array}{cc}\bar{\pi} & \frac{0}{\pi} \\
\frac{\tilde{\sigma}}{0}\end{array}\right|$ & 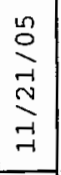 & 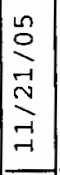 & 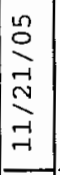 & 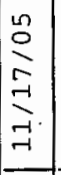 & 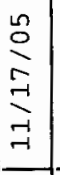 & 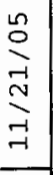 & $\begin{array}{l}n \\
\stackrel{2}{2} \\
\stackrel{-}{N} \\
\stackrel{-}{-1} \\
-1\end{array}$ \\
\hline 号 & $\begin{array}{l}0 \\
\\
\end{array}$ & $\frac{0}{a}$ & \begin{tabular}{|l|}
0 \\
$\overrightarrow{9}$ \\
\end{tabular} & 品 & $\begin{array}{l}0 \\
\overrightarrow{0} \\
\end{array}$ & or & $\frac{\sigma}{9}$ \\
\hline$\left|\begin{array}{ll}\frac{1}{0} & \frac{1}{+} \\
\frac{2}{2} & \frac{d}{0} \\
\bar{\sigma} & 0 \\
\omega & \frac{0}{5}\end{array}\right|$ & 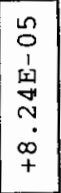 & $\begin{array}{l}n \\
0 \\
1 \\
1 \\
0 \\
01 \\
\dot{m} \\
+ \\
\end{array}$ & \begin{tabular}{|c|}
0 \\
0 \\
1 \\
1 \\
1 \\
0 \\
0 \\
$o$ \\
+ \\
\end{tabular} & $\begin{array}{l}n \\
0 \\
1 \\
1 \\
1 \\
0 \\
0 \\
\dot{+} \\
+\end{array}$ & \begin{tabular}{|l|}
$n$ \\
0 \\
1 \\
1 \\
0 \\
0 \\
0 \\
+ \\
+ \\
\end{tabular} & 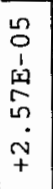 & \begin{tabular}{l}
$n$ \\
0 \\
1 \\
1 \\
1 \\
\multicolumn{1}{c}{} \\
0 \\
0 \\
-1 \\
+
\end{tabular} \\
\hline 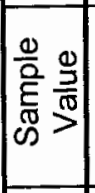 & \begin{tabular}{|l|}
\multicolumn{1}{|c}{} \\
1 \\
1 \\
1 \\
0 \\
0 \\
0 \\
+ \\
\end{tabular} & 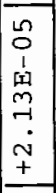 & \begin{tabular}{|l|}
$\overrightarrow{0}$ \\
1 \\
1 \\
1 \\
0 \\
0 \\
$\dot{p}$ \\
+ \\
\end{tabular} & \begin{tabular}{|c|}
0 \\
0 \\
1 \\
1 \\
0 \\
$\infty$ \\
0 \\
1 \\
\end{tabular} & \begin{tabular}{|l|}
0 \\
0 \\
1 \\
1 \\
0 \\
0 \\
0 \\
+ \\
\end{tabular} & 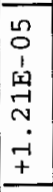 & \begin{tabular}{|l|}
0 \\
0 \\
1 \\
1 \\
0 \\
0 \\
0 \\
0 \\
+ \\
\\
\end{tabular} \\
\hline 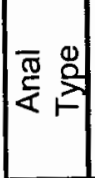 & $\stackrel{\nexists}{\stackrel{N}{D}}$ & $\begin{array}{l}\stackrel{n}{m} \\
\tilde{D} \\
\vec{D}\end{array}$ & 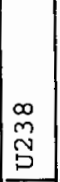 & \begin{tabular}{|l|}
$\infty$ \\
$m$ \\
$\sim$ \\
$⿱ 乛 龰$ \\
\end{tabular} & 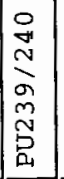 & 壳 & $\begin{array}{l}0 \\
\stackrel{0}{2} \\
\stackrel{D}{D}\end{array}$ \\
\hline 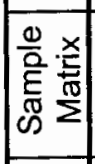 & $\begin{array}{l} \\
n \\
0 \\
.1 \\
\\
\\
0 \\
0 \\
0 \\
\end{array}$ & \begin{tabular}{|l|}
0 \\
0 \\
0 \\
-1 \\
+ \\
4 \\
0 \\
0 \\
\end{tabular} & 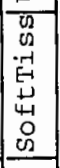 & \begin{tabular}{|l|}
0 \\
0 \\
-7 \\
-1 \\
4 \\
0 \\
0 \\
0 \\
\end{tabular} & \begin{tabular}{|c|} 
\\
0 \\
0 \\
-4 \\
\\
4 \\
0 \\
0 \\
0 \\
\end{tabular} & \begin{tabular}{|l|}
0 \\
02 \\
0 \\
-1 \\
4 \\
4 \\
0 \\
0 \\
0 \\
\end{tabular} & 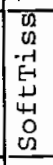 \\
\hline 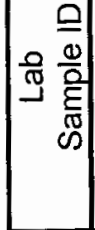 & 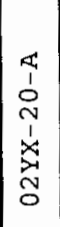 & 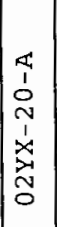 & 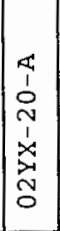 & 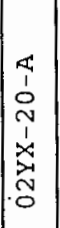 & 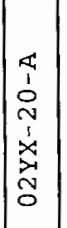 & 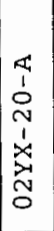 & 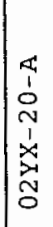 \\
\hline 骂 & \begin{tabular}{|c|c}
0 & 0 \\
0 \\
1 \\
1 \\
0 \\
1 \\
1 \\
0
\end{tabular} & $\mid \begin{array}{l}0 \\
0 \\
0 \\
1 \\
12 \\
1 \\
c 2 \\
c\end{array}$ & \begin{tabular}{|l}
0 \\
0 \\
0 \\
1 \\
0 \\
1 \\
02 \\
2
\end{tabular} & \begin{tabular}{|l|}
0 \\
0 \\
0 \\
1 \\
0 \\
1 \\
0 \\
\end{tabular} & $\mid \begin{array}{l}0 \\
0 \\
0 \\
1 \\
0 \\
0 \\
0 \\
02 \\
\end{array}$ & $\mid \begin{array}{l}n \\
0 \\
0 \\
1 \\
1 \\
1 \\
1 \\
02\end{array}$ & $\begin{array}{l}0 \\
0 \\
0 \\
1 \\
1 \\
0 \\
1 \\
0 \\
\end{array}$ \\
\hline
\end{tabular}

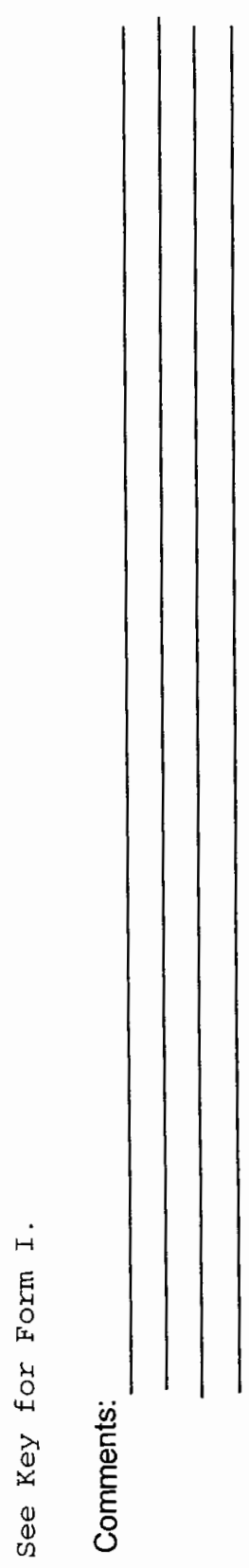




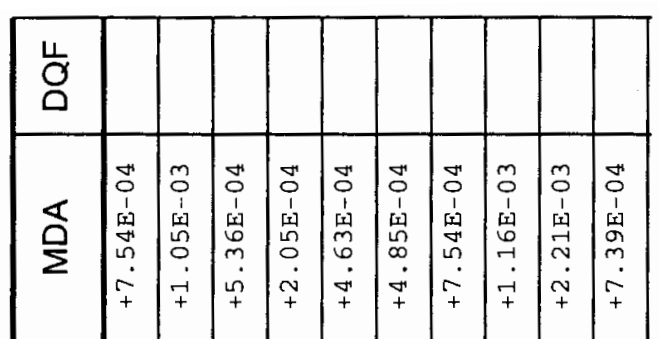

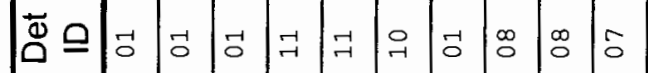

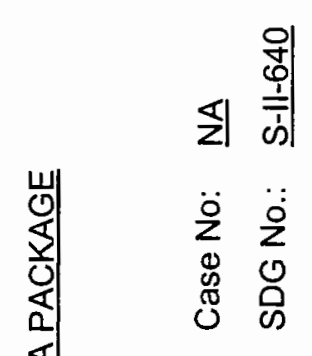

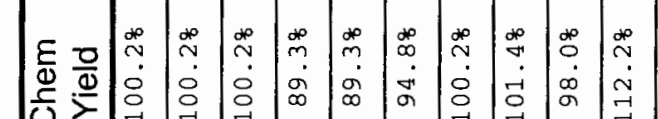

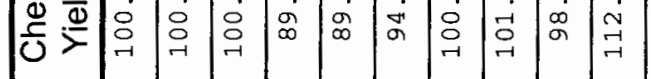

雨

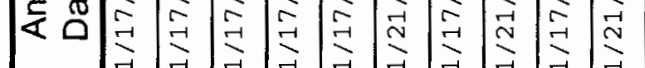

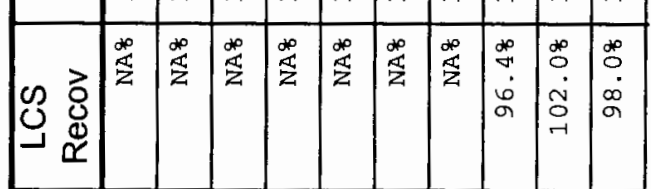

牙

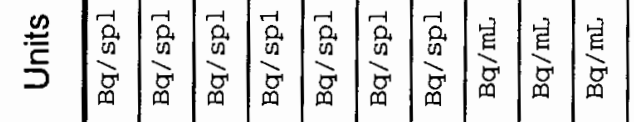

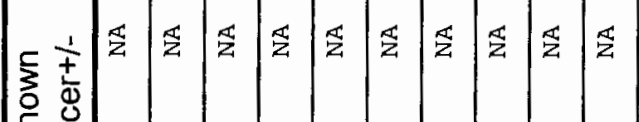

至

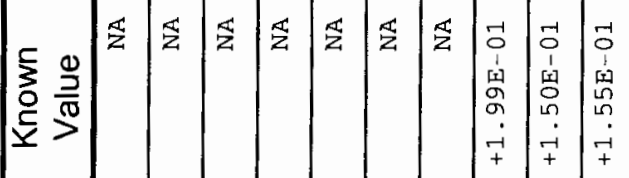

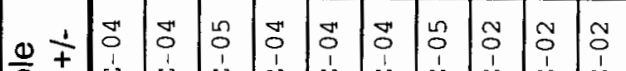

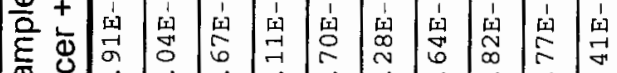

忍

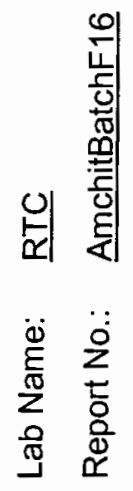

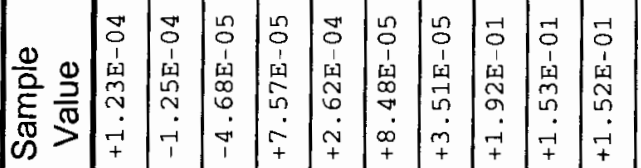

\begin{tabular}{|c|c|c|c|c|c|c|c|c|c|c|}
\hline$\frac{\stackrel{0}{0}}{\stackrel{2}{E}}$ & 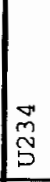 & 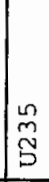 & \begin{tabular}{|l}
$\infty$ \\
$\stackrel{\infty}{S}$ \\
\end{tabular} & 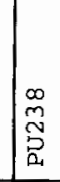 & 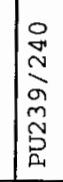 & $\mid \begin{array}{l}\vec{z} \\
\tilde{N} \\
\tilde{y}\end{array}$ & $\mid \begin{array}{l}6 \\
\stackrel{D}{S}\end{array}$ & $\begin{array}{l}\infty \\
m \\
\sim \\
\Gamma\end{array}$ & 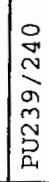 & 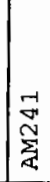 \\
\hline 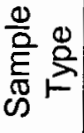 & 蛮 & 裉 & 萱 & 宫 & 总 & $\mid \begin{array}{l}\text { 覓 } \\
\end{array}$ & 学 & 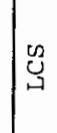 & $\begin{array}{l}\mathscr{y} \\
y \\
y\end{array}$ & 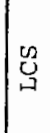 \\
\hline 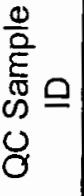 & 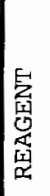 & 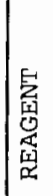 & 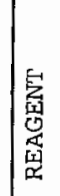 & 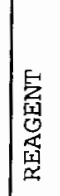 & 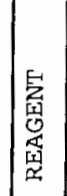 & 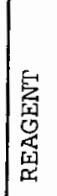 & 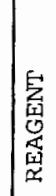 & 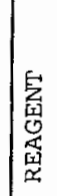 & 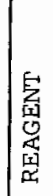 & 畜 \\
\hline
\end{tabular}



Project: $\quad$ Alpha Analysis for Amchitka Island (Batch 16)

Laboratory: RTC

Report \#: AmchitBatchF16

SDG\#: $\quad$ S-II-640

\section{Summary of 2 and 3 sigma activities}

Below are the results for U234, U235, U236, U238, Pu238, Pu239/240, and Am241 for Batch 13 from the Amchitka Island Project that had a result/uncertainty ratio of 2 or more (uncertainty@one sigma).

\begin{tabular}{|c|c|c|c|c|c|c|}
\hline $\begin{array}{l}\text { Customer } \\
\text { ID }\end{array}$ & Lab ID & Isotope & $\begin{array}{c}\text { Result } \\
\mathrm{Bq} / \mathrm{g}\end{array}$ & $\begin{array}{c}\text { Uncertainty } \\
\mathrm{Bq} / \mathrm{g}\end{array}$ & MDA & $\begin{array}{c}\text { Result/ } \\
\text { Uncertainty }\end{array}$ \\
\hline S-II-640 & $02 Y X-01-A$ & U234 & 4.43E-04 & 9.56E-05 & 3.30E-05 & 4.6 \\
\hline S-11-640 & $02 Y X-01-A$ & U238 & $4.26 E-04$ & $9.91 E-05$ & 5.14E-05 & 4.3 \\
\hline S-II-641 & $02 Y X-02-A$ & U234 & $4.60 \mathrm{E}-04$ & $9.41 E-05$ & 3.83E-05 & 4.9 \\
\hline S-1I-641 & $02 Y X-02-A$ & U238 & $3.98 E-04$ & 8.98E-05 & 3.57E-05 & 4.4 \\
\hline S-11-642 & 02YX-03-A & U234 & $3.12 E-04$ & 7.58E-05 & 3.78E-05 & 4.1 \\
\hline S-1I-642 & $02 Y X-03-A$ & U238 & 2.76E-04 & 7.21E-05 & 3.78E-05 & 3.8 \\
\hline S-II-643 & $02 Y X-04-A$ & U234 & $2.78 \mathrm{E}-03$ & 4.19E-04 & 5.08E-05 & 6.6 \\
\hline S-11-643 & $02 Y X-04-A$ & U235 & $1.42 E-04$ & $5.23 E-05$ & 4.35E-05 & 2.7 \\
\hline S-11-643 & $02 Y X-04-A$ & U238 & $2.28 \mathrm{E}-03$ & $3.93 E-04$ & 4.64E-05 & 5.8 \\
\hline S-11-644 & 02YX-05-A & U234 & 4.68E-04 & $9.66 \mathrm{E}-05$ & 3.73E-05 & 4.8 \\
\hline S-II-644 & 02YX-05-A & U238 & 4.66E-04 & $1.02 \mathrm{E}-04$ & 3.73E-05 & 4.6 \\
\hline S-1I-645 & $02 Y X-06-A$ & U234 & 8.72E-04 & $1.41 \mathrm{E}-04$ & 2.90E-05 & 6.2 \\
\hline S-1I-645 & $02 Y X-06-A$ & U235 & 4.86E-05 & $2.41 E-05$ & 1.31E-05 & 2.0 \\
\hline S-II-645 & 02YX-06-A & U238 & 7.38E-04 & $1.35 \mathrm{E}-04$ & 2.65E-05 & 5.5 \\
\hline S-11-646 & 02YX-07-A & U234 & 7.46E-04 & $1.41 \mathrm{E}-04$ & 4.11E-05 & 5.3 \\
\hline S-II-646 & 02YX-07-A & U238 & $7.18 \mathrm{E}-04$ & $1.46 \mathrm{E}-04$ & $3.76 \mathrm{E}-05$ & 4.9 \\
\hline S-II-647 & 02YX-08-A & U234 & $6.70 \mathrm{E}-04$ & $1.26 \mathrm{E}-04$ & 4.10E-05 & 5.3 \\
\hline S-11-647 & $02 Y X-08-A$ & U238 & 5.34E-04 & $1.13 \mathrm{E}-04$ & 3.48E-05 & 4.7 \\
\hline S-11-648 & $02 Y X-09-A$ & $\mathrm{AM} 241$ & $5.15 \mathrm{E}-03$ & 5.57E-04 & 8.83E-05 & 9.2 \\
\hline$S \cdot 11-648$ & 02YX-09-A & PU239/240 & 8.08E-03 & 8.67E-04 & 3.77E-05 & 9.3 \\
\hline S-11-648 & 02YX-09-A & U234 & 8.88E-04 & $1.49 \mathrm{E}-04$ & 3.44E-05 & 6.0 \\
\hline S-II-648 & 02YX-09-A & U235 & 8.08E-05 & 3.33E-05 & 1.56E-05 & 2.4 \\
\hline S-1I-648 & 02YX-09-A & U238 & $7.52 E-04$ & 1.42E-04 & 3.15E-05 & 5.3 \\
\hline S-S-649 & $02 Y X-10-A$ & U234 & 5.06E-04 & 1.02E-04 & 3.79E-05 & 5.0 \\
\hline S-S-649 & $02 Y X-10-A$ & U238 & 4.43E-04 & 9.94E-05 & 4.79E-05 & 4.5 \\
\hline
\end{tabular}




\section{Alpha Analysis for Amchitka Island (Batch 16) Summary of 2 and 3 sigma activities \\ page 2}

\begin{tabular}{|c|c|c|c|c|c|c|}
\hline $\begin{array}{l}\text { Customer } \\
\text { ID }\end{array}$ & Lab ID & Isotope & $\begin{array}{c}\text { Result } \\
\mathrm{Bq} / \mathrm{g}\end{array}$ & $\begin{array}{l}\text { Uncertainty } \\
\text { Bq/g }\end{array}$ & MDA & $\begin{array}{c}\text { Result/ } \\
\text { Uncertainty }\end{array}$ \\
\hline S-S-651 & $02 Y X-12-A$ & U234 & 7.83E-03 & $1.01 \mathrm{E}-03$ & 5.31E-05 & 7.8 \\
\hline S-S-651 & $02 Y X-12-A$ & U235 & $4.25 \mathrm{E}-04$ & 1.05E-04 & $4.55 \mathrm{E}-05$ & 4.0 \\
\hline S-S-651 & $02 Y X-12-A$ & U238 & $7.56 \mathrm{E}-03$ & $1.11 \mathrm{E}-03$ & $4.85 E-05$ & 6.8 \\
\hline S-S-652 & $02 Y X-13-A$ & U234 & $9.49 E-04$ & $1.58 \mathrm{E}-04$ & 3.82E-05 & 6.0 \\
\hline S-S-652 & $02 Y X-13-A$ & U238 & 8.44E-04 & $1.57 \mathrm{E}-04$ & 3.34E-05 & 5.4 \\
\hline S-S-653 & $02 Y X-14-A$ & U234 & $6.75 E-04$ & $1.30 \mathrm{E}-04$ & 4.39E-05 & 5.2 \\
\hline S-S-653 & $02 Y X-14-A$ & U238 & 5.83E-04 & $1.24 \mathrm{E}-04$ & 3.73E-05 & 4.7 \\
\hline S-S-654 & $02 Y X-15-A$ & U234 & $5.60 \mathrm{E}-04$ & $1.14 E-04$ & 4.15E-05 & 4.9 \\
\hline S-S-654 & $02 Y X-15-A$ & U238 & $5.26 \mathrm{E}-04$ & $1.15 E-04$ & 3.79E-05 & 4.6 \\
\hline S-S-655 & $02 Y X-16-A$ & U234 & 8.44E-04 & $1.53 \mathrm{E}-04$ & 4.03E-05 & 5.5 \\
\hline S-S-655 & $02 Y X-16-A$ & U238 & $7.99 \mathrm{E}-04$ & $1.59 \mathrm{E}-04$ & 4.03E-05 & 5.0 \\
\hline S.S.656 & $02 Y X-17-A$ & U234 & $5.09 E-04$ & $1.04 \mathrm{E}-04$ & 4.19E-05 & 4.9 \\
\hline S-S-656 & $02 Y X-17-A$ & U238 & 4.69E-04 & $1.03 E-04$ & $3.17 E-05$ & 4.6 \\
\hline S-S-657 & $02 Y X-18-A$ & U234 & 5.67E-04 & 1.19E-04 & 4.71E-05 & 4.8 \\
\hline S-S-657 & $02 Y X-18-A$ & U238 & 5.27E-04 & 1.21E-04 & $5.68 E-05$ & 4.4 \\
\hline S-S-658 & $02 Y X-19-A$ & U234 & 4.09E-04 & 8.69E-05 & 4.10E-05 & 4.7 \\
\hline S-S-658 & $02 Y X-19-A$ & U238 & $4.01 E-04$ & $9.03 E-05$ & $3.58 \mathrm{E}-05$ & 4.4 \\
\hline S-S-659 & $02 Y X-20-A$ & U234 & $3.60 \mathrm{E}-04$ & 8.24E-05 & 4.27E-05 & 4.4 \\
\hline S-S-659 & $02 Y X-20-A$ & U238 & 3.87E-04 & 9.07E-05 & 3.63E-05 & 4.3 \\
\hline
\end{tabular}

All known sources of uncertainty are included in the uncertainty term. There may be unknown sources of uncertainty that are not accounted for. If the result/uncertainty ratio is more than 3 , we have a degree of confidence that the result is positive (i.e. the result is statistically different than zero). A result with the result/uncertainty ratio between 2 and 3 is the first indication that an isotope may be present and further investigation may be warranted.

As with any good science no single data point is used in important decisions (results need to be reproducible). 


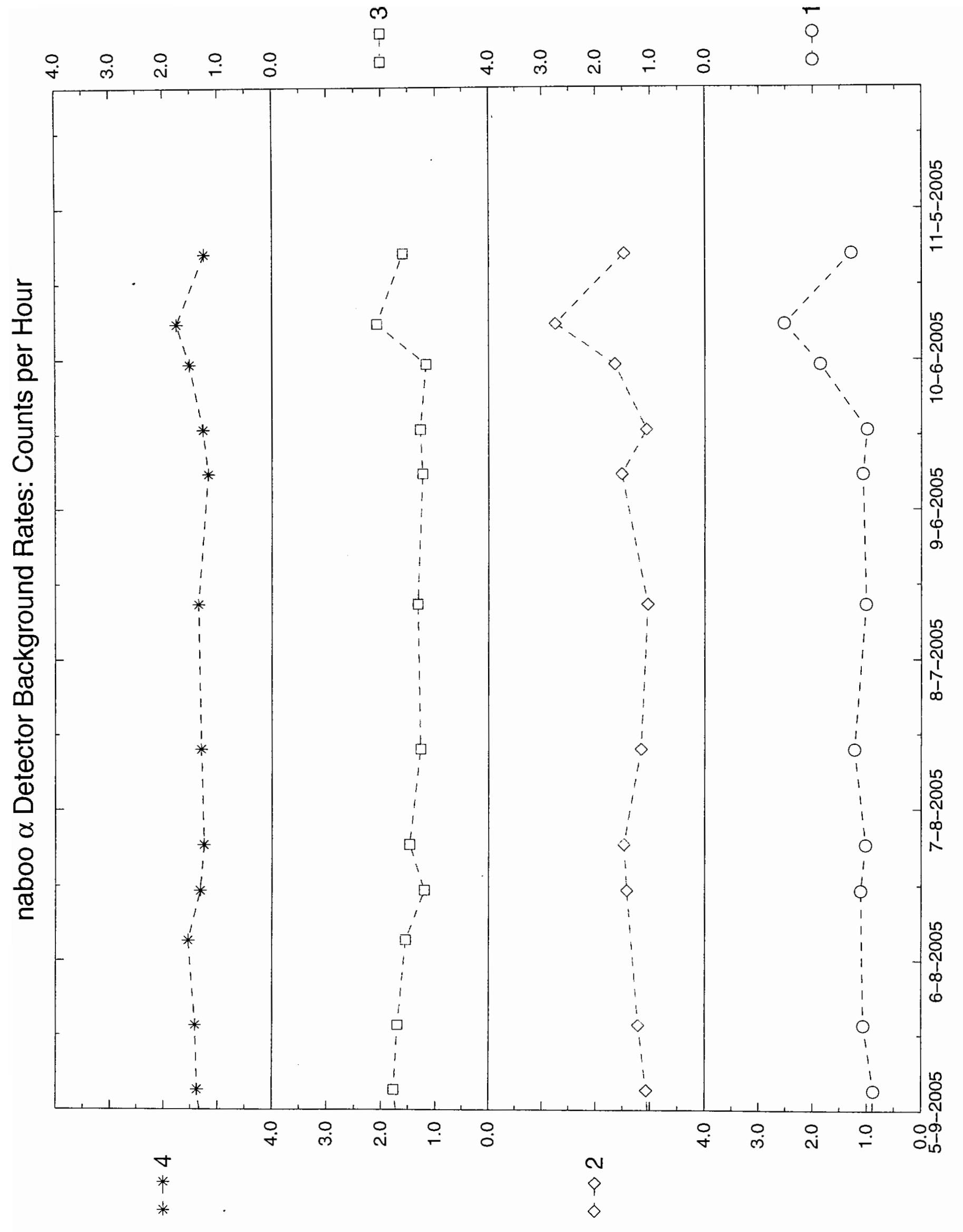




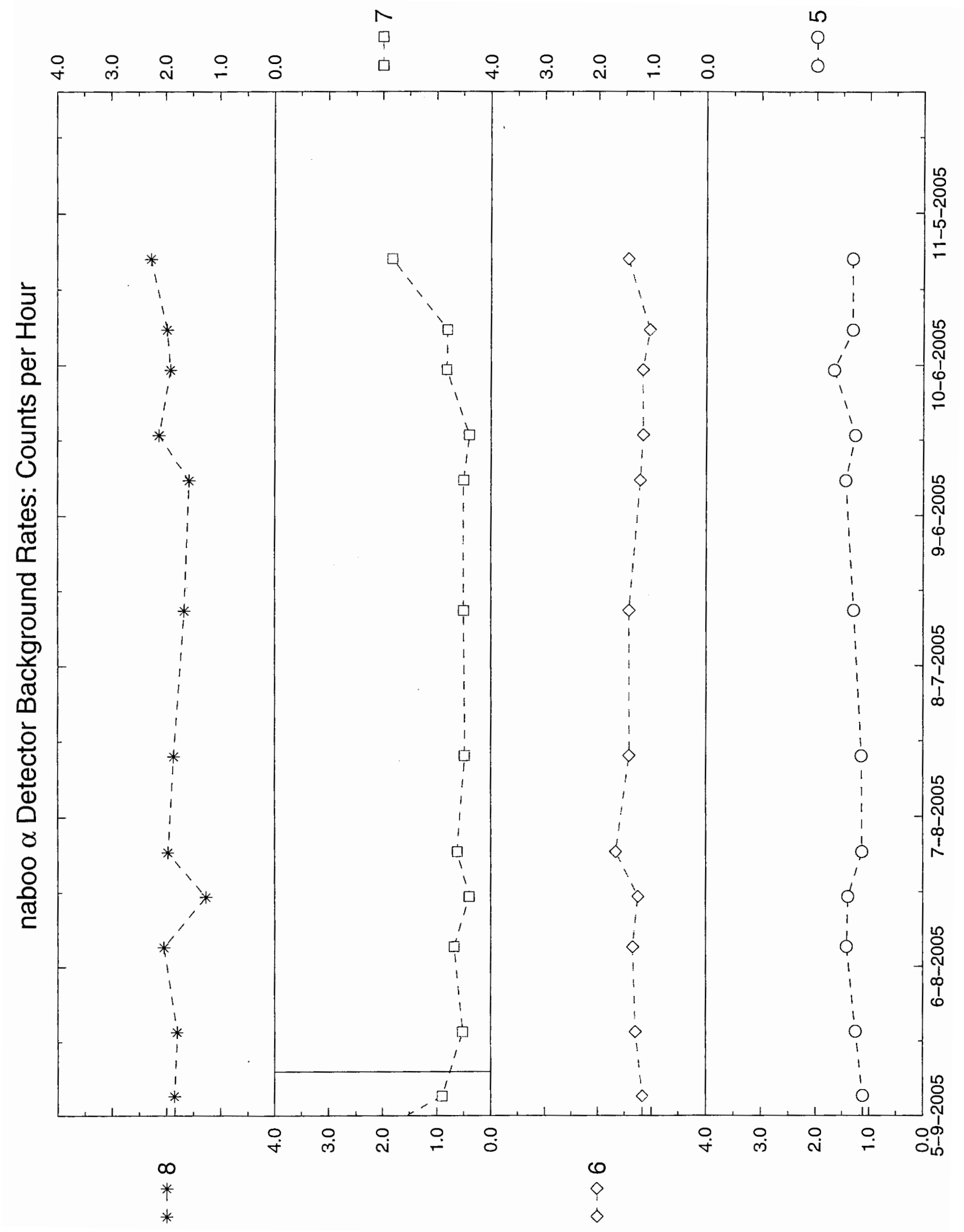




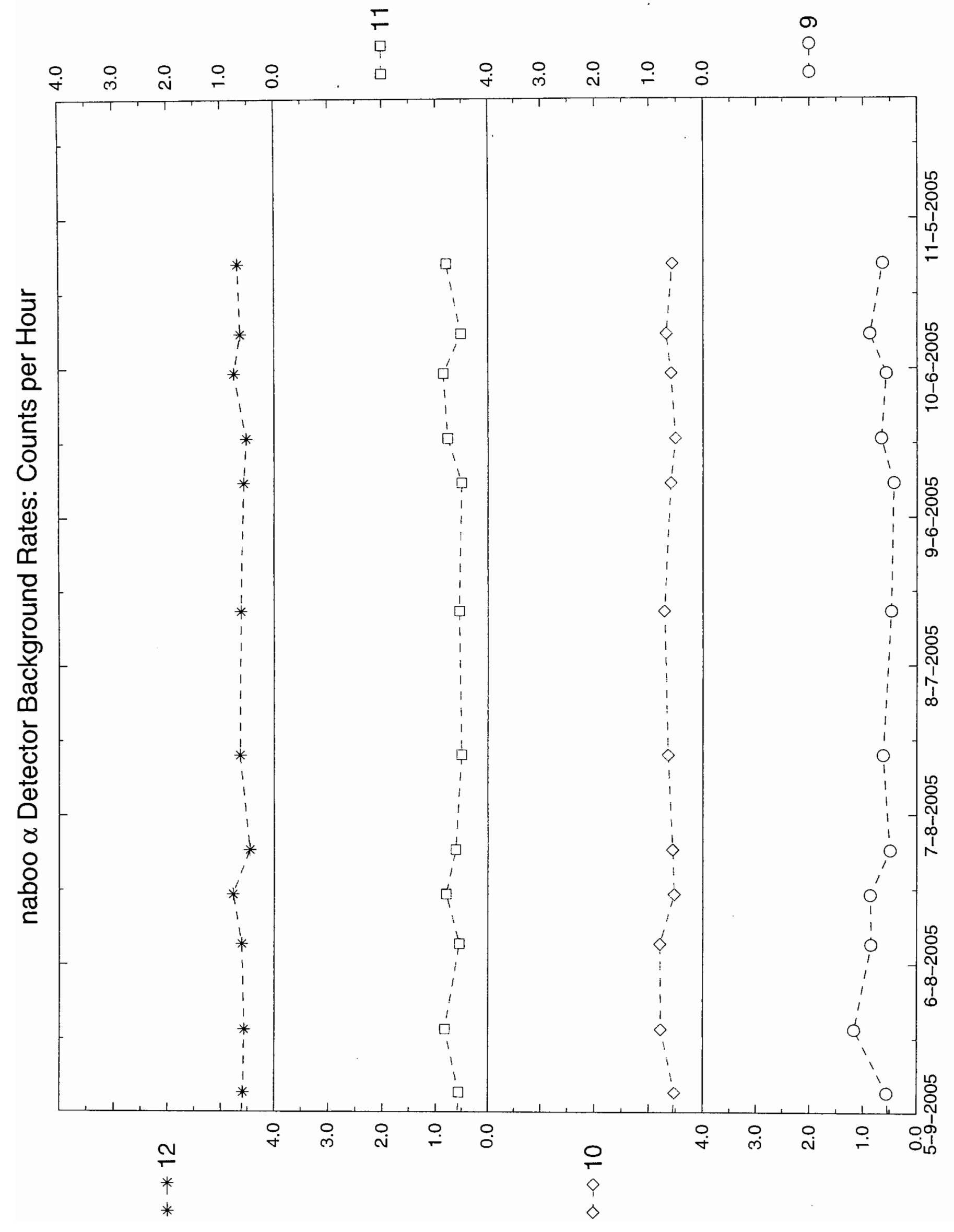




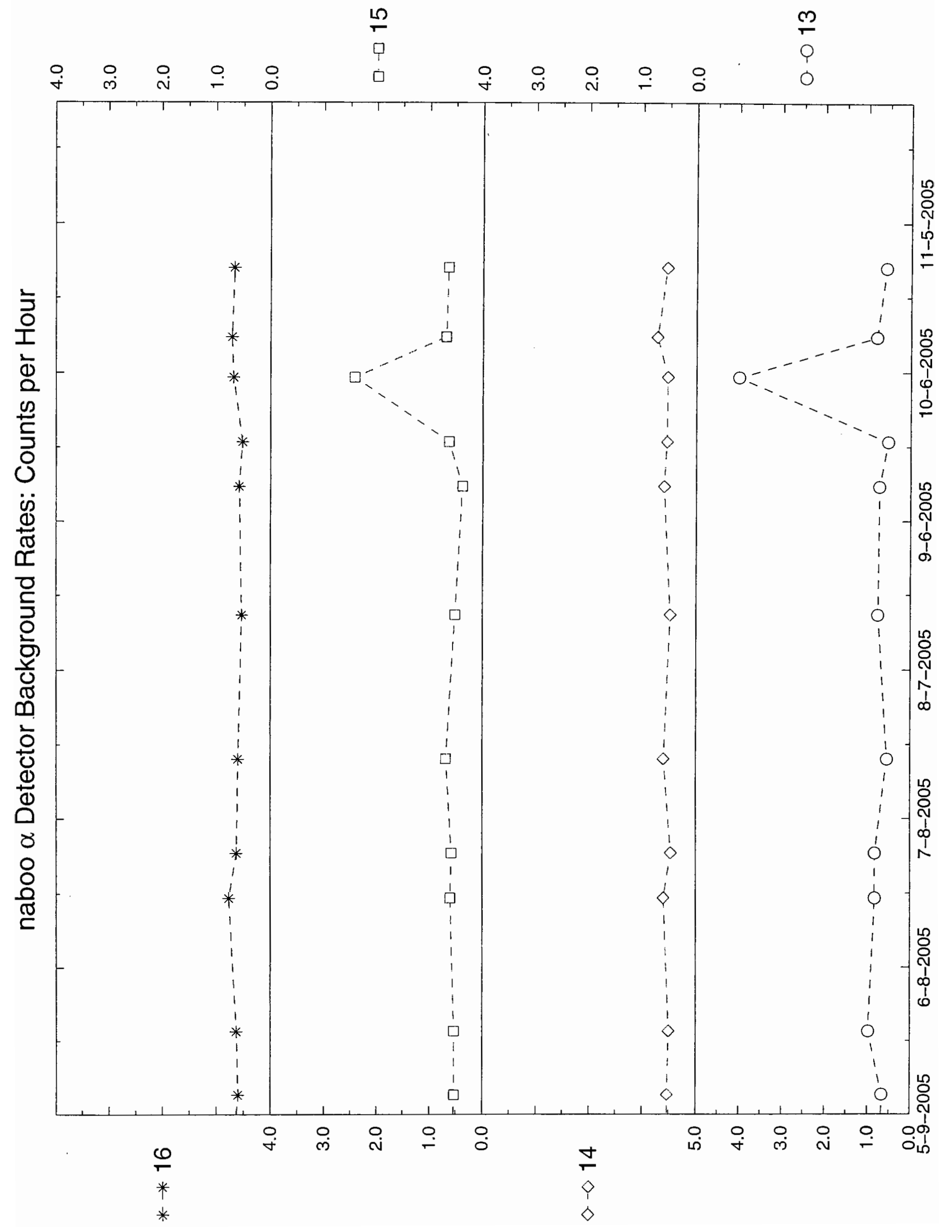




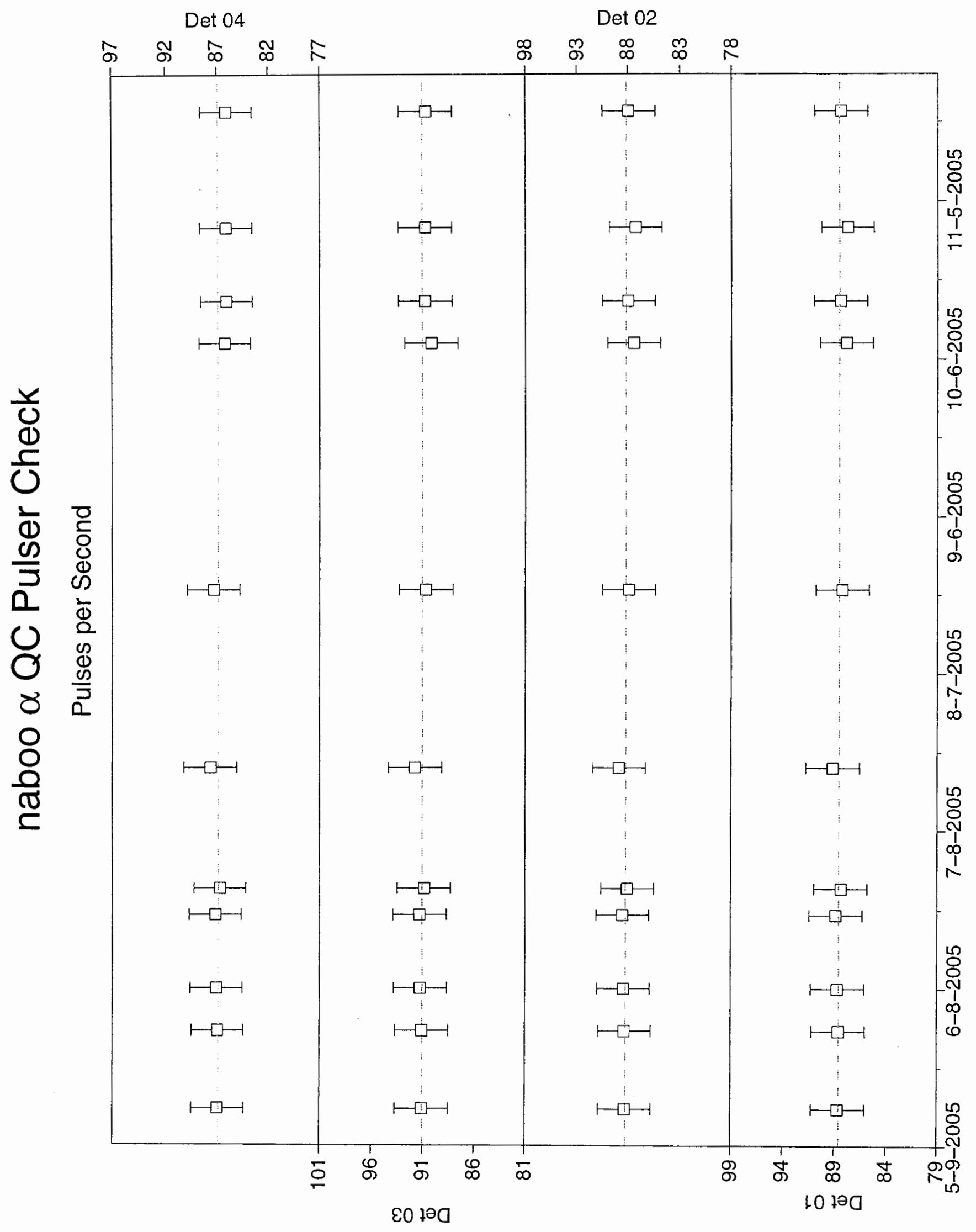




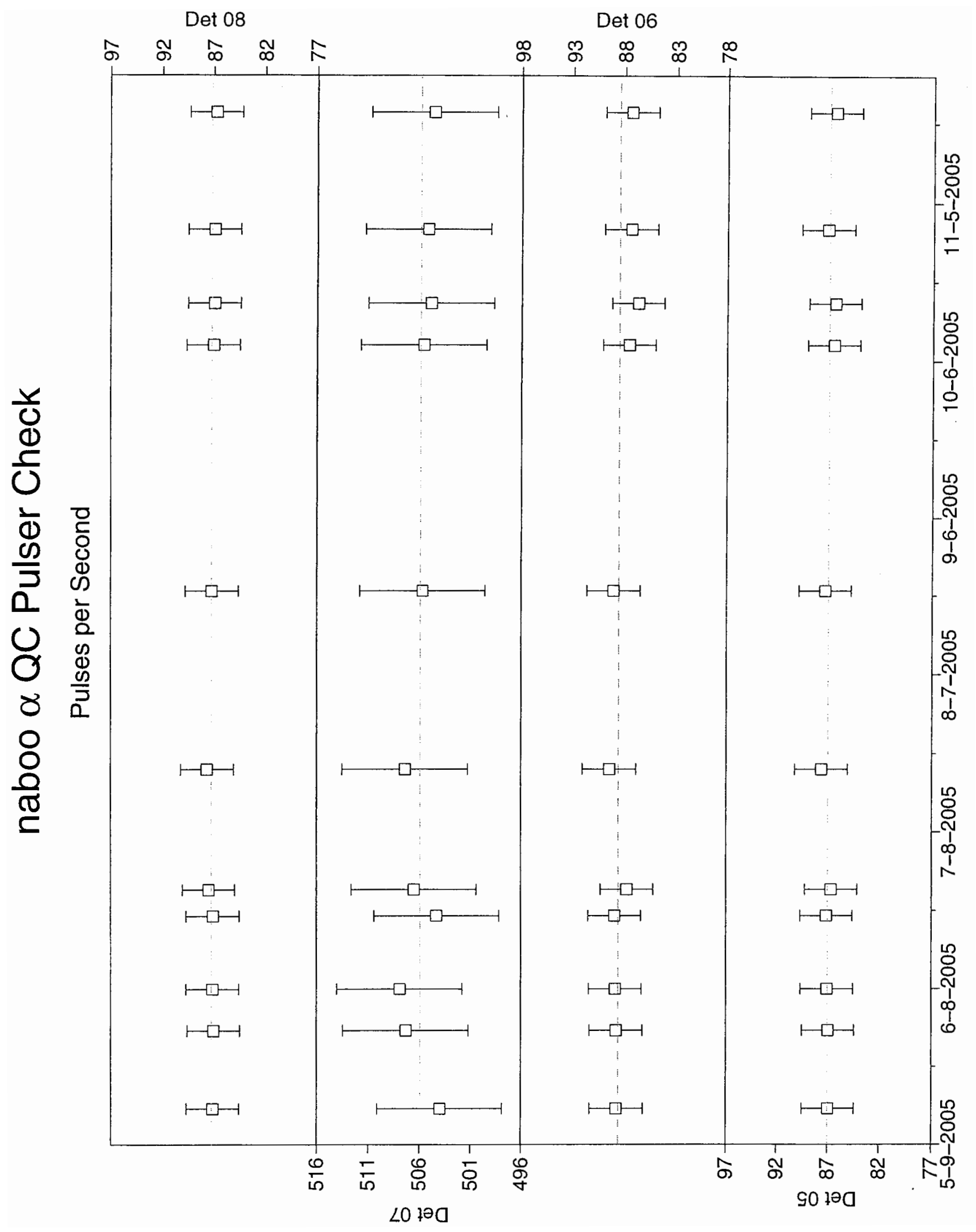




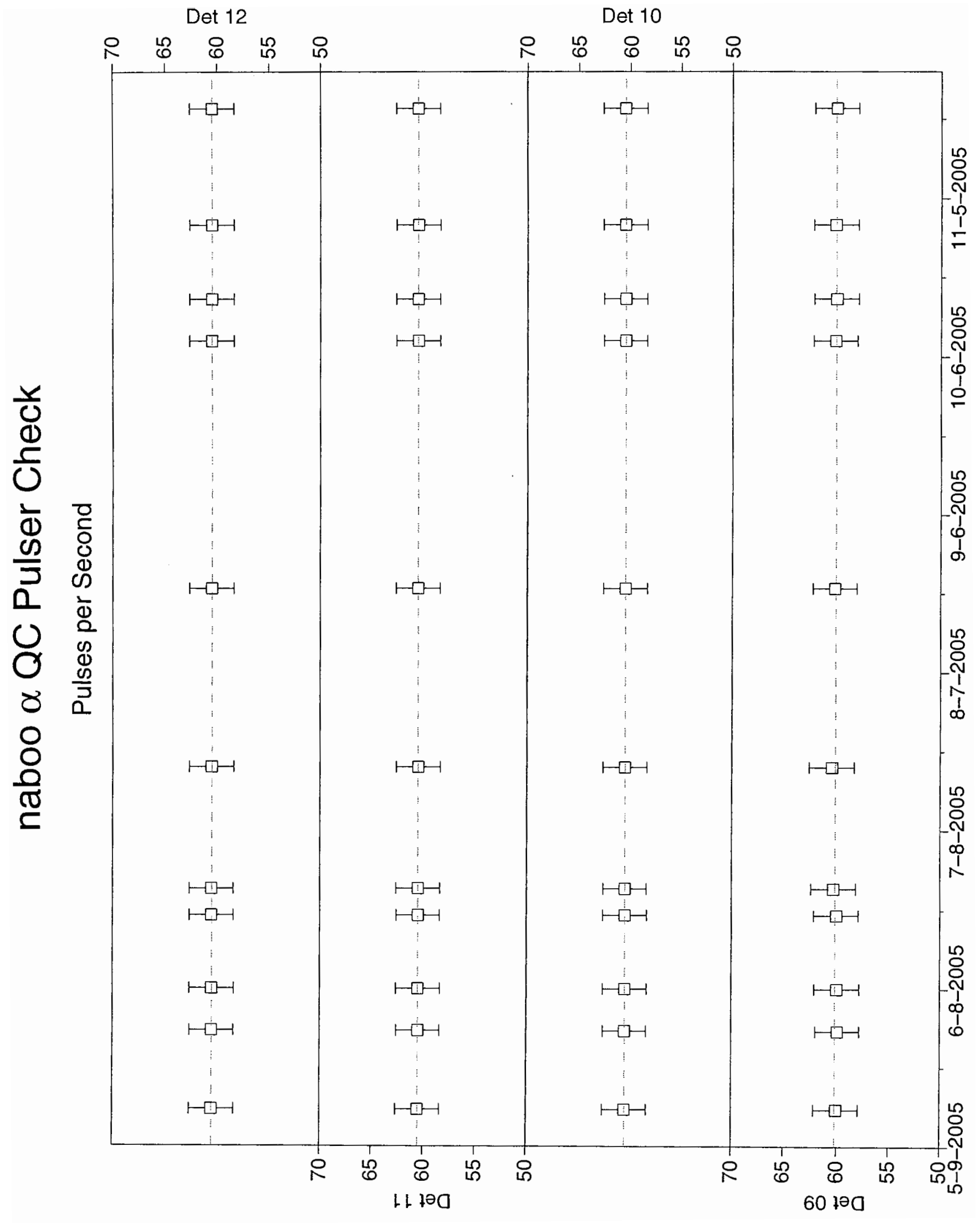




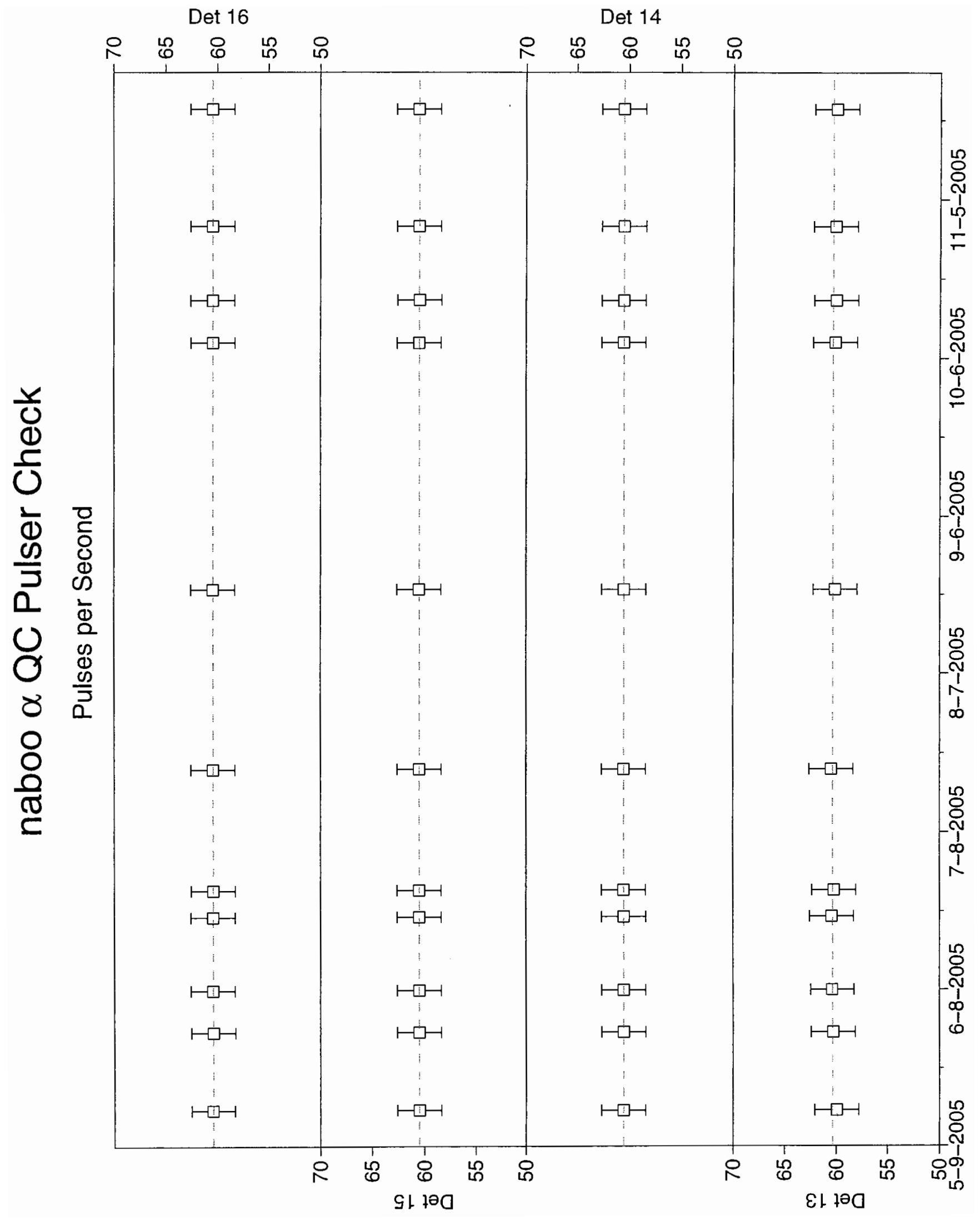




\section{Mixed Analyte Performance Evaluation Program}

Laboratory Results

LOCKO3 RADIATION MEASUREMENTS LABORATORYIAEDL

Sample ID: $\quad$ MAPEP-05-MaW13

INEEL

\begin{tabular}{|c|c|c|c|c|c|c|c|c|}
\hline & Idaho Falls & 10 & & $83415-7111$ & & & & \\
\hline Analyte & Result & $\begin{array}{c}\text { Ref } \\
\text { Value }\end{array}$ & Flag & $\begin{array}{l}\text { Flag } \\
\text { Text } \\
\end{array}$ & $\begin{array}{l}\text { Bias } \\
(\%)\end{array}$ & $\begin{array}{c}\text { Acceptance } \\
\text { Range } \\
\end{array}$ & $\begin{array}{l}\text { Unc } \\
\text { Value }\end{array}$ & $\begin{array}{l}\text { Unc. } \\
\text { Flag Units }\end{array}$ \\
\hline Barium & NR & 2.63 & & & & $1.84-3.42$ & & \\
\hline Beryllium & NR & 0.417 & & & & $0.29-0.54$ & & \\
\hline Cadmium & NR & 0.303 & & & & $0.21-0.39$ & & \\
\hline Chromium & NR & 1.67 & & & & $1.17 \cdot 2.17$ & & \\
\hline Copper & NR & 1.92 & & & & $1.34-2.50$ & & \\
\hline Lead & NR & 1.21 & & & $\therefore$ & $0.85-1.57$ & & \\
\hline Mercury & NR & 0.102 & & & & $0.07-0.13$ & & \\
\hline Nickel & NR & 0.968 & & & & $0.68-1.26$ & & \\
\hline Selenium & NR & 0.362 & & & & $0.25-0.47$ & & \\
\hline Uranium-238 & NR & 0.268 & & & & & & \\
\hline Vanadium & NR & 3.67 & & & & $2.57-4.77$ & & \\
\hline Zinc & NR & 1.33 & & & & $0.93-1.73$ & & \\
\hline Americium-241 & 1.50 & 1.72 & A & & -12.8 & $1.20 \cdot 2.24$ & .10 & $(B q / L)$ \\
\hline Cesium-134 & 118 & 127 & A & & -7.1 & $88.90-165.10$ & 9 & $(\mathrm{~Bq} / \mathrm{L})$ \\
\hline Cesium-137 & 318 & 332 & A & & -4.2 & $232.40-431.60$ & 23 & $(\mathrm{~Bq} / \mathrm{L})$ \\
\hline Cobalt-57 & 224 & 227 & A & & -1.3 & $158.90-295.10$ & 16 & $(\mathrm{~Bq} / \mathrm{L})$ \\
\hline Cobalt-60 & 250 & 251 & A & & -0.4 & $175.70-326.30$ & 18 & $(\mathrm{~Bq} / \mathrm{L})$ \\
\hline Hydrogen-3 & 320 & 280 & A & & 14.3 & $196.00-364.00$ & 50 & $(B q / L)$ \\
\hline |ron-55 & 81.3 & 75.9 & A & & 7.1 & $53.13-98.67$ & 4.9 & $(\mathrm{~Bq} / \mathrm{L})$ \\
\hline Manganese-54 & 323 & 331 & A & & -2.4 & $231.70-430.30$ & 23 & $(\mathrm{~Bq} / \mathrm{L})$ \\
\hline Nickel-63 & 4.4 & 9.0 & A & 17 & & & 1.7 & $(\mathrm{~Bq} / \mathrm{L})$ \\
\hline Plutonium-238 & 0.011 & 0.018 & A & 17 & & & 0.015 & $(\mathrm{~Bq} / \mathrm{L})$ \\
\hline Plutonium-239/240 & 2.36 & 2.4 & A & & -1.7 & $1.68 \cdot 3.12$ & .18 & $(\mathrm{~Bq} / \mathrm{L})$ \\
\hline Strontium-90 & -.2 & & A & & & & .4 & $(\mathrm{~Bq} / \mathrm{L})$ \\
\hline Technetium-99 & $N R$ & 42.9 & & & & $30.03-55.77$ & & \\
\hline Uranium-234/233 & 3.23 & 3.24 & A & & -0.3 & $2.27-4.21$ & .23 & $(\mathrm{~Bq} / \mathrm{L})$ \\
\hline Uranium-238 & 3.33 & 3.33 & A & & 0.0 & $2.33-4.33$ & .23 & $(\mathrm{~Bq} / \mathrm{L})$ \\
\hline Zinc-65 & 509 & 496 & $A$ & & 2.6 & $347.20-644.80$ & 37 & $(\mathrm{~Bq} / \mathrm{L})$ \\
\hline
\end{tabular}




\section{Mixed Analyte Performance Evaluation Program \\ Laboratory Results}

\begin{tabular}{|c|c|c|c|c|c|c|c|c|}
\hline \multirow{5}{*}{$\begin{array}{l}\text { Sample } L \mathrm{D} \\
\text { LOCK03 }\end{array}$} & \multirow{2}{*}{\multicolumn{8}{|c|}{ MAPEP-05-GrW13 }} \\
\hline & & & & & & & & \\
\hline & \multirow{2}{*}{\multicolumn{8}{|c|}{$\begin{array}{l}\text { RADIATION MEASUREMENTS LABORATORYIAEDL } \\
\text { INEEL }\end{array}$}} \\
\hline & & & & & & & & \\
\hline & Idaho Falls & ID & \multicolumn{2}{|c|}{ 83415-7111 } & \multirow[b]{2}{*}{$\begin{array}{c}\text { Acceptance } \\
\text { Range }\end{array}$} & \multirow[b]{2}{*}{$\begin{array}{l}\text { Unc } \\
\text { Value }\end{array}$} & \multirow[b]{2}{*}{$\begin{array}{l}\text { Unc. } \\
\text { Flag }\end{array}$} & \multirow[b]{2}{*}{ Units } \\
\hline Analyte & Result & $\begin{array}{c}\text { Ref } \\
\text { Value }\end{array}$ & Flag & $\begin{array}{l}\text { Bias } \\
(\%)\end{array}$ & & & & \\
\hline Gross alpha & .038 & 0.525 & $A$ & -92.8 & $0.000-1.050$ & .006 & & $(\mathrm{~Bq} / \mathrm{L})$ \\
\hline Gross beta & 1.53 & 1.67 & A & -8.4 & $0.835-2.505$ & .06 & & $(B \mathrm{~g} / \mathrm{L})$ \\
\hline
\end{tabular}

Gross Alpha Flags:

$A=$ Result acceptable, Bias $<=+1-100 \%$ with a statistically positive result at two standard deviations

(Result/Uncertainty $>2$, i.e., the range encompassing the result, plus or minus the total uncertainty at two standard deviations, does not include zero).

$N=$ Result not acceptable, Bias $>+1-100 \%$ or the reported result is not statistically positive at two standard deviations (ResulfUncertainty $<=2$, i.e., the range encompassing the result, plus or minus the total uncertainty at two standard deviations, includes zero).

Gross Beta Flags:

$A=$ Result acceptable, Bias $<=+1-50 \%$ with a statistically positive result at two standard deviations

(Result/Uncertainty $>2$, i.e., the range encompassing the result, plus or minus the total uncertainty at two standard deviations, does not include zero).

$\mathrm{N}=$ Result not acceptable, Bias $>+1-50 \%$ or the reported result is not statistically positive at two standard deviations (ResulvUncertainty $<=2$, i.e., the range encompassing the result, plus or minus the total uncertainty at two standard deviations, includes zero).

Flags: $\quad L=$ Uncertainty potentially too low (for infomation purposes only)

$\mathrm{H}=$ Uncertainty potentially too high (for information purposes only)

$\mathrm{FP}=$ False Positive

$\mathrm{FN}=$ False Negative

NR $=$ Not Reported 


\section{Mixed Analyte Performance Evaluation Program}

\section{Laboratory Results}

LOCK03 RADIATION MEASUREMENTS LABORATORYIAEDL Sample ID: MAPEP-05-MaS13 INEEL

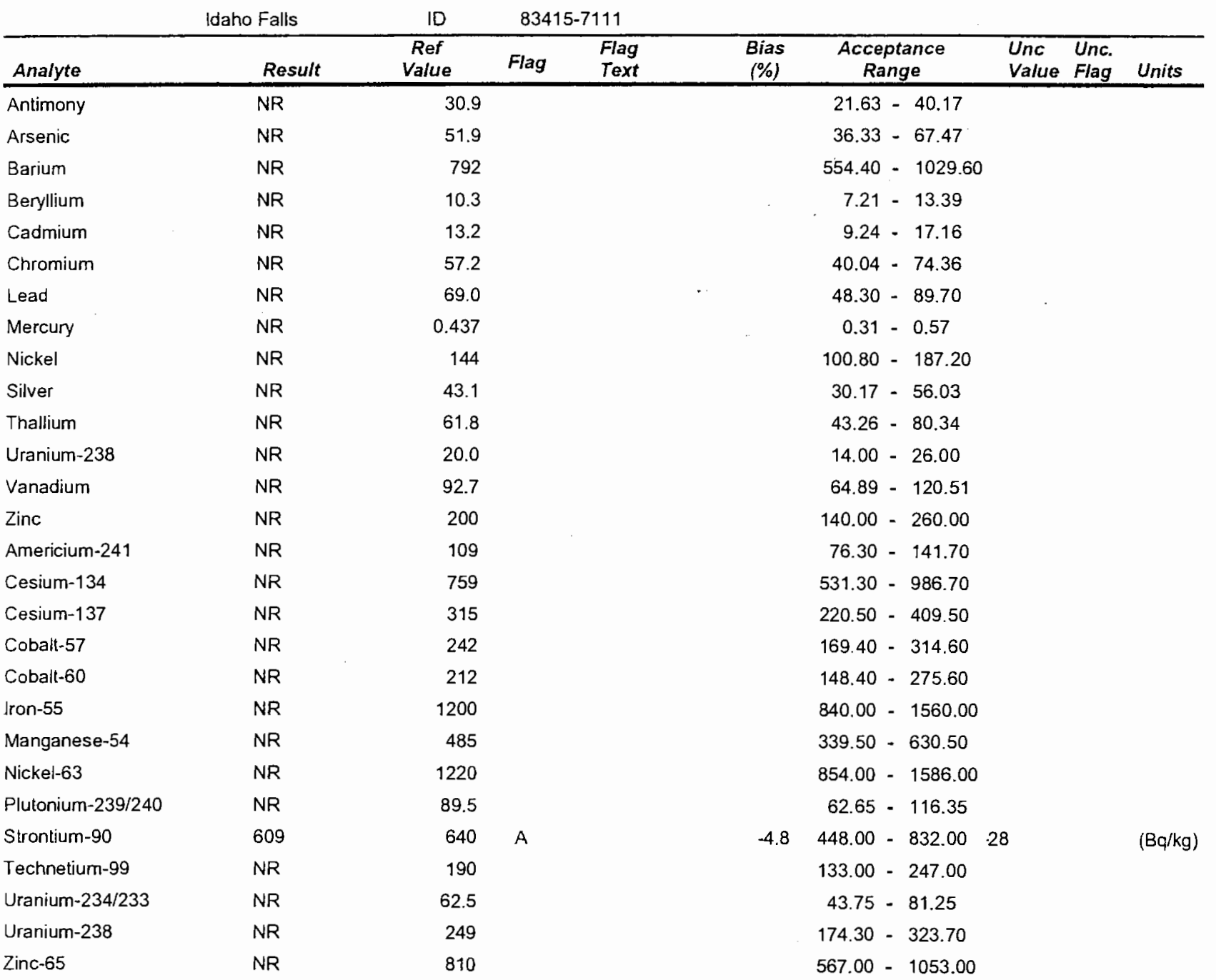

\footnotetext{
Flags: $\quad A=$ Result acceptable $\quad$ Bias $<=20 \%$

$W=$ Result acceptable with warning $20 \%<$ Bias $<=30 \%$
Flag Text 1 - False Positive
2 - False Negative
4 - Sensitivity Evaluation
5 - Total Metal
6 - Not Evaluated
7 - DL > CLP Limit
9 - Check QL
10 - Check Isomer
11 - False Positive Test, Value Not Reported
14 - Solubility Issue
15 - Refractory
16 - Reported zero uncertainty
17 - NOT DETECTED, reported a statistical zerc result
18 - Sensitivity evaluation, value not reported




\section{Mixed Analyte Performance Evaluation Program}

\section{Laboratory Results}

RADIATION MEASUREMENTS LABORATORYIAEDL

LOCKO3 INEEL

Sample ID: $\quad$ MAPEP-03-W11

\begin{tabular}{|c|c|c|c|c|c|c|c|c|}
\hline Falls & ID & 834 & & & & & & \\
\hline Result & $\begin{array}{c}\text { Ref } \\
\text { Value }\end{array}$ & Flag & $\begin{array}{l}\text { Flag } \\
\text { Text } \\
\end{array}$ & $\begin{array}{l}\text { Sias } \\
(\%)\end{array}$ & $\begin{array}{c}\text { Acceptance } \\
\text { Range }\end{array}$ & $\begin{array}{l}\text { Unc } \\
\text { Value } \\
\end{array}$ & $\begin{array}{l}\text { Unc. } \\
\text { Flag } \\
\end{array}$ & Units \\
\hline NR & 0.1296 & & & & $0.09-0.17$ & & & \\
\hline NR & 0.0537 & & & & $0.04-0.07$ & & & \\
\hline NR & 0.541 & & & & $0.38-0.70$ & & & \\
\hline NR & 0.0985 & & & . & $0.07-0.13$ & & & \\
\hline NR & 0.0799 & & & & $0.06-0.10$ & & & \\
\hline NR & 0.803 & & & & $0.56-1.04$ & & & \\
\hline NR & 0.894 & & & & $0.63-1.16$ & & & \\
\hline NR & 0.495 & & & & $0.35=0.64$ & & & \\
\hline NR & 0.06981 & & & & $0.05-0.09$ & & & \\
\hline NR & 2.088 & & & & $1.46-2.74$ & & & \\
\hline NR & 0.1968 & & & & $0.14-0.26$ & & & \\
\hline NR & 0.001417 & & & & & & & \\
\hline NR & 0.1954 & & & & $0.14-0.25$ & & & \\
\hline NR & 1.2 & & & & $0.84-1.56$ & & & \\
\hline NR & 1.037 & & & & $0.73-1.35$ & & & \\
\hline \multicolumn{9}{|l|}{ NR } \\
\hline 310 & 322 & A & & -3.7 & $225.40-418.60$ & 20 & & $(B q / L)$ \\
\hline 122 & 124 & A & & -1.6 & $86.80-161.20$ & 9 & & $(B q / L)$ \\
\hline 170 & 173 & A & & -1.7 & $121.10-224.90$ & 12 & & $(\mathrm{~Bq} / \mathrm{L})$ \\
\hline 125 & 121.8 & A & & 2.6 & $85.26-158.34$ & 9 & & $(\mathrm{~Bq} / \mathrm{L})$ \\
\hline NR & 379 & & & & $265.30-492.70$ & & & \\
\hline 123 & 131 & A & & -6.1 & $91.70-170.30$ & 6 & & $(B q / L)$ \\
\hline 154 & 155 & A & & -0.6 & $108.50-201.50$ & 12 & & $(B q / L)$ \\
\hline 81.3 & 73.7 & A & & 10.3 & $51.59-95.81$ & 4.7 & & $(B q / L)$ \\
\hline 1.28 & 1.49 & A & & -14.1 & $1.04-1.94$ & .11 & & $(\mathrm{~Bq} / \mathrm{L})$ \\
\hline 2.26 & 2.39 & A & & -5.4 & $1.67-3.11$ & 0.09 & & $(B q / L)$ \\
\hline 15.4 & 17.7 & A & & -13.0 & $12.39-23.01$ & 0.7 & & $(\mathrm{~Bq} / \mathrm{L})$ \\
\hline NR & 28.8 & & & & $20.16-37.44$ & & & \\
\hline 2.39 & 2.35 & A & & 1.7 & $1.64-3.05$ & 0.12 & & $(B q / L)$ \\
\hline 2.35 & 2.43 & A & & -3.3 & $1.70-3.16$ & .12 & & $(\mathrm{~Bq} / \mathrm{L})$ \\
\hline 330 & 320 & A & & 3.1 & $224.00-416.00$ & 30 & & $(\mathrm{~Bq} / \mathrm{L})$ \\
\hline
\end{tabular}

Flags: $\quad \begin{aligned} & A=\text { Result acceptable } \quad \text { Bias } \leqslant=20 \% \\ & \quad W=\text { Result acceptable with warning } 20 \%<\text { Bias }<=30 \%\end{aligned}$

$\mathrm{N}=$ Result not acceptable $\quad$ Bias $>30 \%$

$L=$ Uncertainty potentially too low (for infomation purposes only)

$H=$ Uncertainty potentially too high (for information purposes only)

$Q=$ Particapant should evaluate reported value

$\mathrm{QL}=$ Quantitation Limit

$\mathrm{RW}=$ Report Warning

NR $=$ Not Reported

NOTE 1: False Positive Test, Value Not Reported 


\section{Mixed Analyte Performance Evaluation Program}

\section{Laboratory Results}

\section{LOCKO3 INEEL}

RADIATION MEASUREMENTS LABORATORYIAEDL

Sample ID: $\quad$ MAPEP-03-S10

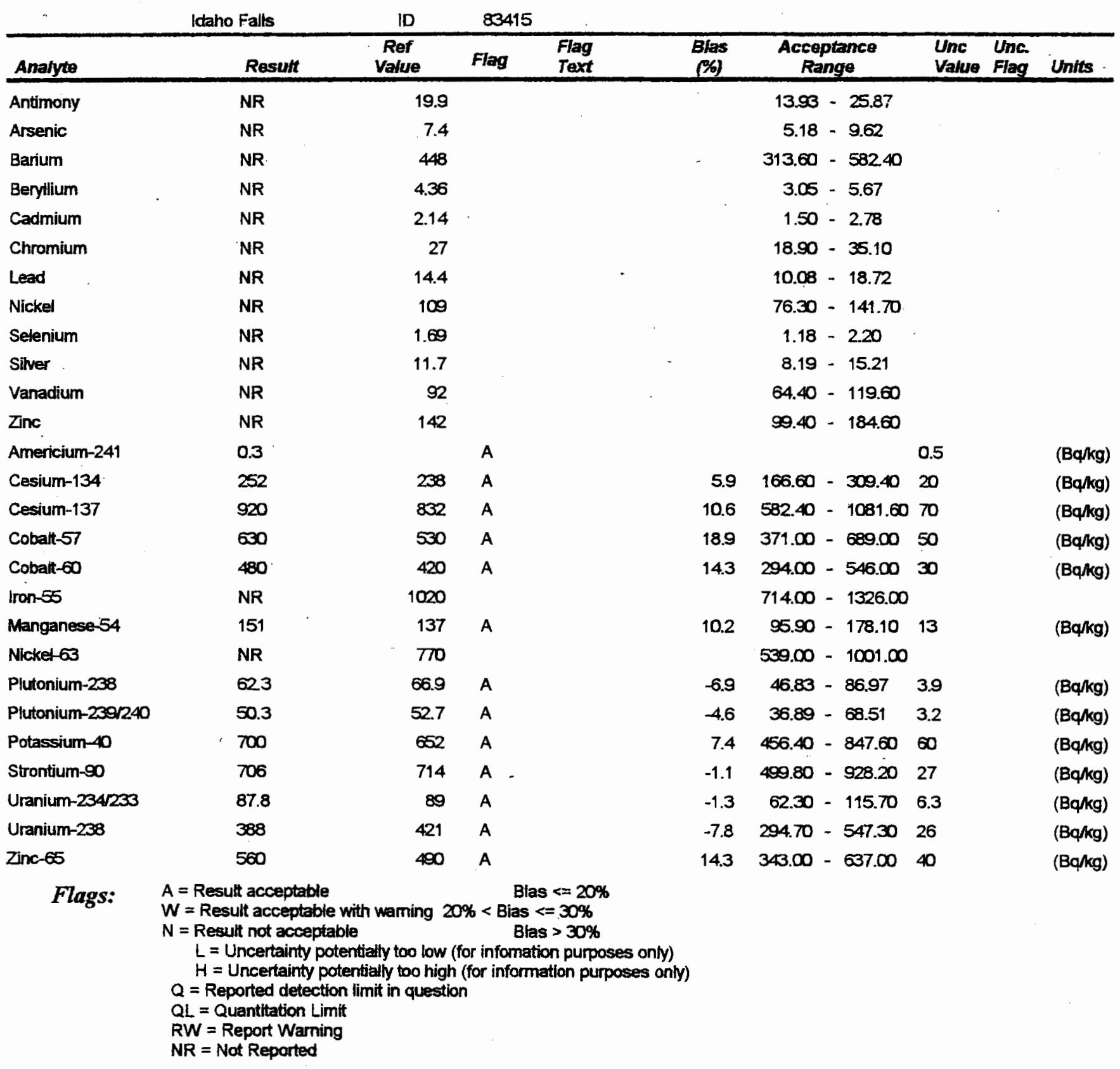




\section{Mixed Analyte Performance Evaluation Program}

\section{Laboratory Results}

Sample ID: MAPEP-02-W10

RADIATION MEASUREMENTS LABORATORY/AEDL

LOCKO3 INEEL

Idaho Falls ID $\quad 83415$

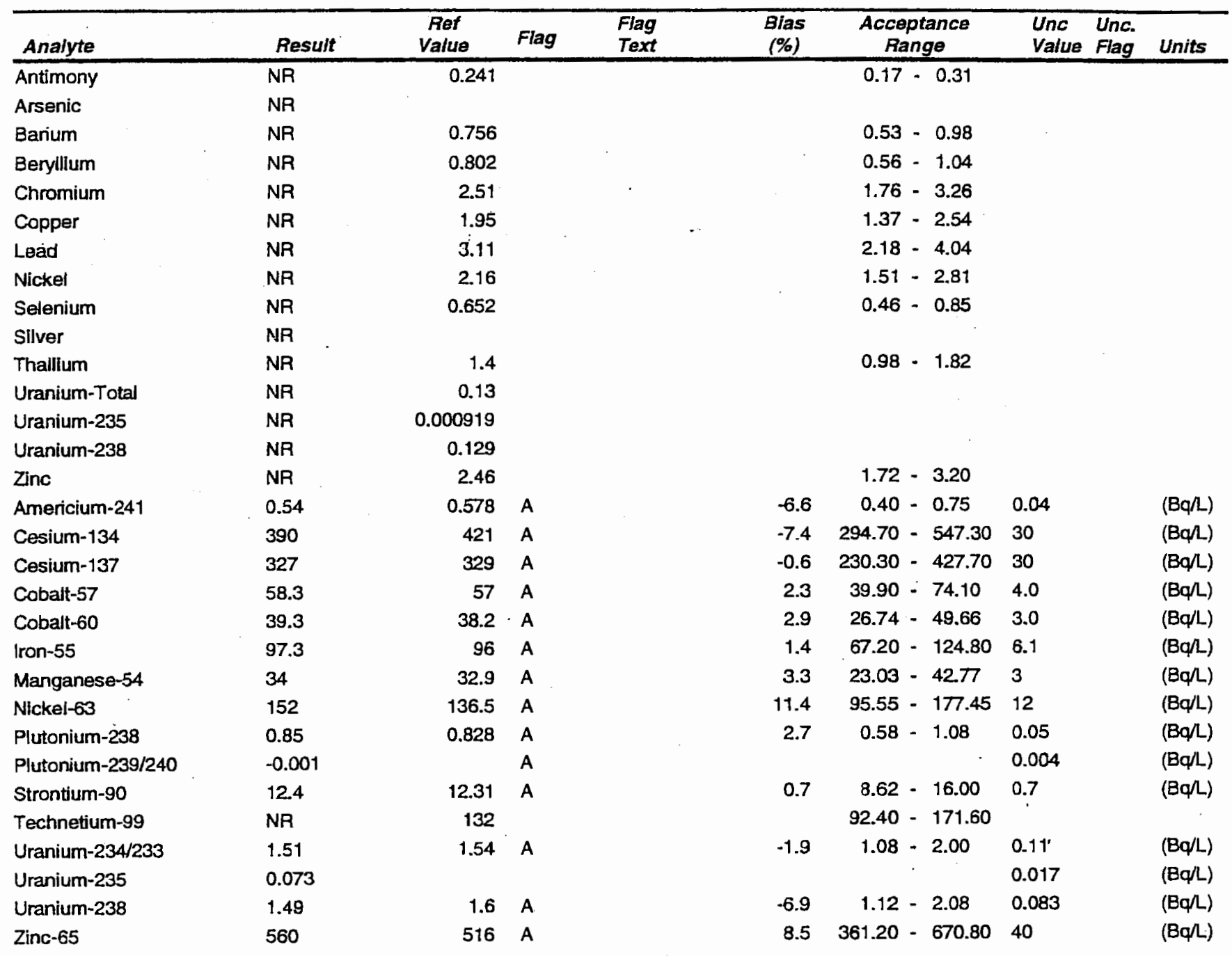

Flags: $\quad A=$ Result acceptable $\quad$ Bias $<=20 \%$

$\mathrm{W}=$ Result acceptable with warning $20 \%<$ Bias $<=30 \%$

$N=$ Result not acceptable . Bias $>30 \%$

$\mathrm{L}=$ Uncertainty potentially too low (for infomation purposes only)

$\mathrm{H}=$ Uncertainty potentially too high (for information purposes only)

QL $=$ Quantitation Limit

NR = Not.Reported

NOTE 1: False Positive Test, Value Not Reported 


\section{Mixed Analyte Performance Evaluation Program}

\section{Laboratory Results}

Sample ID: MAPEP-02-S9

RADIATION MEASUREMENTS LABORATORYIAEDL

LOCKO3 INEEL

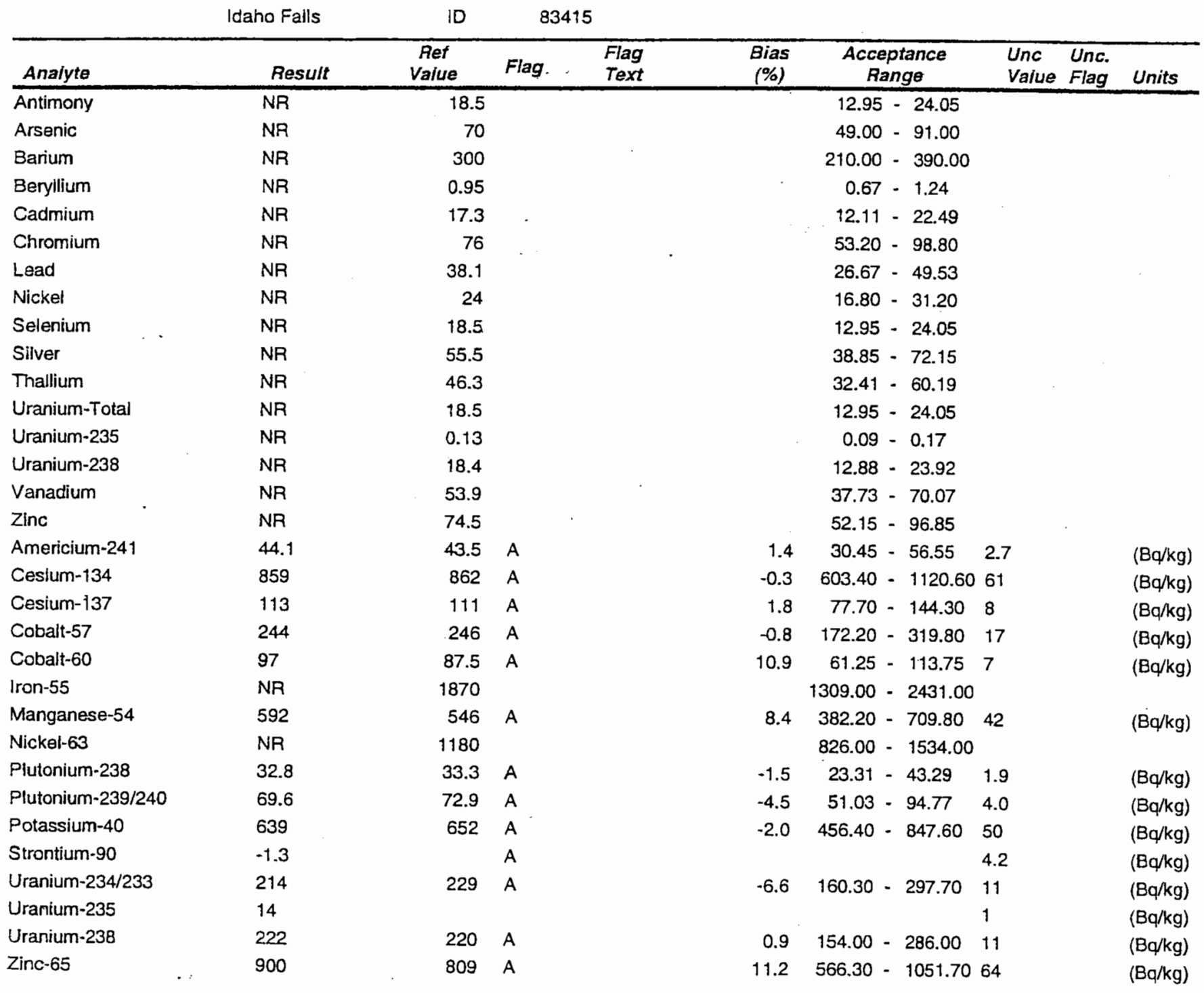

Flags: $\quad A=$ Result acceptable

Bias $<=20 \%$

$\mathrm{W}=$ Result acceptable with warning $20 \%<$ Bias $<=30 \%$

$\mathrm{N}=$ Result not acceptable

Bias $>30 \%$

$\mathrm{L}=$ Uncertainty potentially too low (for infomation purposes only)

$\mathrm{H}=$ Uncertainty potentially too high (for information purposes only)

$\mathrm{QL}=$ Detection Limit

$\mathrm{RW}=$ Report Warning

NR $=$ Not Reported 
QAP 0303

EML-621

June 2003

\section{QAP 58 Results by Laboratory}

Lab: EG INEEL TRA Radioanalytical Laboratory, Scoville

\begin{tabular}{|c|c|c|c|c|c|c|c|c|}
\hline $\begin{array}{l}\text { No. } \\
\text { Test }\end{array}$ & Radionuclide & $\begin{array}{l}\text { Reported } \\
\text { Value }\end{array}$ & $\begin{array}{l}\text { Reported } \\
\text { Error }\end{array}$ & $\begin{array}{l}\text { EvIL } \\
\text { Value }\end{array}$ & $\begin{array}{l}\text { EML } \\
\text { Error }\end{array}$ & $\frac{\text { Reported }}{\text { EML }}$ & Evaluation & $\begin{array}{l}\text { QAP } 56 \\
\text { Evaluation }\end{array}$ \\
\hline
\end{tabular}

Matrix: Al Air Filter Bq / filter

$\begin{array}{llrlrllll}1 & \text { AM241 } & 0.282 & 0.017 & 0.340 & 0.040 & 0.829 & \text { W } & A \\ 1 & \text { CO60 } & 38.000 & 1.000 & 33.500 & 0.870 & 1.134 & \text { W } & \text { A } \\ 1 & \text { CS137 } & 118.000 & 7.000 & 99.700 & 2.300 & 1.184 & \text { W } & A \\ 1 & \text { MN54 } & 53.000 & 1.000 & 43.800 & 1.130 & 1.210 & \text { W } & \text { A } \\ 1 & \text { PU238 } & 0.536 & 0.027 & 0.520 & 0.010 & 1.031 & \mathrm{~A} & \text { W } \\ 1 & \text { PU239 } & 0.345 & 0.018 & 0.330 & 0.010 & 1.045 & \mathrm{~A} & \mathrm{~A} \\ 1 & \text { U234 } & 0.221 & 0.014 & 0.240 & 0.003 & 0.921 & \mathrm{~A} & \mathrm{~A} \\ 1 & \text { U238 } & 0.224 & 0.014 & 0.240 & 0.010 & 0.933 & \mathrm{~A} & \mathrm{~A}\end{array}$

Matrix: SO Soil $\mathrm{Bq} / \mathrm{kg}$

$\begin{array}{ll}1 & \mathrm{AC} 228 \\ 1 & \mathrm{~B} 212 \\ 1 & \mathrm{~B} 1214 \\ 1 & \mathrm{CS} 137 \\ 1 & \mathrm{~K} 40 \\ 1 & \mathrm{~PB} 212 \\ 1 & \mathrm{~PB} 214 \\ 1 & \mathrm{~T} 1234\end{array}$

$\begin{array}{rrrr}68.000 & 7.000 & 57.600 & 2.500 \\ 99.000 & 9.000 & 60.600 & 4.000 \\ 77.000 & 5.000 & 67.000 & 2.300 \\ 1672.000 & 11.000 & 1450.000 & 73.000 \\ 688.000 & 48.000 & 636.000 & 33.000 \\ 81.000 & 5.000 & 57.900 & 2.900 \\ 83.000 & 5.000 & 71.100 & 2.300 \\ 224.000 & 21.000 & 127.000 & 7.100\end{array}$

$\begin{array}{lll}1.181 & \text { A } & \text { W } \\ 1.634 & \text { N } & \text { A } \\ 1.149 & \text { A } & \text { A } \\ 1.153 & \text { A } & \text { A } \\ 1.082 & \text { A } & \text { A } \\ 1.399 & \text { N } & \text { A } \\ 1.167 & \text { A } & \text { A } \\ 1.764 & \text { W } & \text { A }\end{array}$

Matrix: VE Vegetation $\mathrm{Bq} / \mathrm{kg}$

$$
\begin{array}{ll}
1 & \text { CO60 } \\
1 & \text { CS } 137
\end{array}
$$$$
10.500
$$$$
435.000
$$$$
1160.000
$$

$$
\begin{aligned}
& 3.000 \\
& 8.000
\end{aligned}
$$

12.100

444.000

1120.000

$$
\begin{array}{r}
0.700 \\
22.000 \\
60.000
\end{array}
$$

0.868
0.980
1.036

$\begin{array}{ll}\text { W } & \text { W } \\ \text { A } & \text { A } \\ \text { A } & \text { A }\end{array}$

Matrix: WA Water $\mathrm{Bq} / \mathrm{L}$

$\begin{array}{ll}1 & \text { AM24! } \\ 1 & \text { CO60 } \\ 1 & \text { CSI34 } \\ 1 & \text { CSI37 } \\ 1 & \text { Gross Alpha } \\ 1 & \text { Gross Beta } \\ 1 & \text { PU238 } \\ 1 & \text { PU239 } \\ 1 & \text { U234 } \\ 1 & \text { U238 }\end{array}$

$$
\begin{array}{r}
2.260 \\
240.000 \\
30.000 \\
64.000 \\
247.000 \\
784.000 \\
3.870 \\
4.570 \\
2.050 \\
2.040
\end{array}
$$

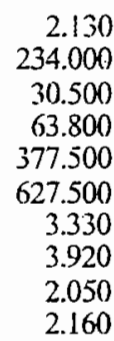

1.061
1.026
0.984
1.003
0.654
1.249
1.162
1.166
1.000
0.944

$\begin{array}{ll}\text { A } & \text { A } \\ \text { A } & \text { A } \\ \text { A } & \text { A } \\ \text { A } & \text { A } \\ \text { W } & \text { A } \\ \text { A } & \text { A } \\ \text { W } & \text { W } \\ \text { W } & \text { W } \\ \text { A } & \text { A } \\ \text { A } & \text { A }\end{array}$

Values for elemental uranium are reported in $\mu \mathrm{g} /$ filter, $g$, or $\mathrm{mL}$. 
EML-618

December 2002

\section{QAP 57 Results by Laboratory}

Lab: EG INEEL TRA Radioanalytical Laboratory, Scoville

\begin{tabular}{llccccc} 
No. & $\begin{array}{c}\text { Reported } \\
\text { Value }\end{array}$ & $\begin{array}{c}\text { Reported } \\
\text { Error }\end{array}$ & $\begin{array}{l}\text { EML } \\
\text { Value }\end{array}$ & $\begin{array}{c}\text { EML } \\
\text { Error }\end{array}$ & $\begin{array}{c}\text { Reported } \\
\text { EML }\end{array}$ Evaluation & QAP 56 \\
Evaluation \\
\hline
\end{tabular}

Matrix: $\mathrm{AI}$ Air Filter $\mathrm{Bq} /$ filter

\begin{tabular}{|c|c|c|c|c|c|c|}
\hline AM241 & 0.195 & 0.014 & 0.191 & 0.004 & 1.023 & A \\
\hline $\mathrm{CO} 60$ & 25.000 & 2.000 & 23.000 & 0.059 & 1.087 & A \\
\hline CS137 & 36.000 & 3.000 & 32.500 & 0.777 & 1.308 & A \\
\hline MN54 & 60.000 & 5.000 & 52.200 & 1.170 & 1.149 & A \\
\hline PU238 & 0.097 & 0.005 & 0.119 & 0.003 & 0.817 & W \\
\hline PU239 & 0.183 & 0.009 & 0.206 & 0.002 & 0.889 & A \\
\hline SR90 & 5.740 & 0.290 & 5.561 & 0.119 & 1.032 & A \\
\hline U234 & 0.218 & 0.009 & 0.228 & 0.006 & 0.958 & A \\
\hline U238 & 0.215 & 0.009 & 0.230 & 0.006 & 0.935 & A \\
\hline
\end{tabular}

Matrix: SO Soil Bq/ $\mathrm{kg}$

$\begin{array}{ll}1 & \text { AC228 } \\ 1 & \text { AM241 } \\ 1 & \text { B1212 } \\ 1 & \text { BI214 } \\ 1 & \text { CS137 } \\ 1 & \text { K40 } \\ 1 & \text { PB212 } \\ 1 & \text { PB214 } \\ 1 & \text { PU238 } \\ 1 & \text { PU239 } \\ 1 & \text { TH234 } \\ 1 & \text { U234 } \\ 1 & \text { U238 }\end{array}$

$\begin{array}{rrr}36.000 & 7.000 & 42.300 \\ 6.870 & 0.690 & 6.767 \\ 47.000 & 20.000 & 45.930 \\ 31.000 & 4.000 & 33.630 \\ 755.000 & 60.000 & 829.330 \\ 600.000 & 41.000 & 637.670 \\ 42.000 & 4.000 & 43.430 \\ 34.000 & 4.000 & 35.200 \\ 18.400 & 1.400 & 19.203 \\ 13.400 & 0.700 & 12.903 \\ 51.000 & 22.000 & 48.400 \\ 47.900 & 2.900 & 42.320 \\ 48.100 & 2.400 & 44.890\end{array}$

1.560
0.301
4.510
1.560
41.580
34.260
2.710
1.510
0.855
0.465
4.830
3.100
3.200

$\begin{array}{lll}0.851 & \mathrm{~W} & \mathrm{~A} \\ 1.015 & \mathrm{~A} & \mathrm{~A} \\ 1.023 & \mathrm{~A} & \mathrm{~A} \\ 0.922 & \mathrm{~A} & \mathrm{~N} \\ 0.910 & \mathrm{~A} & \mathrm{~A} \\ 0.941 & \mathrm{~A} & \mathrm{~N} \\ 0.967 & \mathrm{~A} & \mathrm{~W} \\ 0.966 & \mathrm{~A} & \mathrm{~A} \\ 0.958 & \mathrm{~A} & \\ 1.038 & \mathrm{~A} & \mathrm{~W} \\ 1.054 & \mathrm{~A} & \mathrm{~A} \\ 1.132 & \mathrm{~W} & \mathrm{~A} \\ 1.072 & \mathrm{~A} & \mathrm{~A}\end{array}$

Matrix: VE Vegetation $\mathrm{Bq} / \mathrm{kg}$

$$
\begin{array}{ll}
1 & \text { CO60 } \\
1 & \text { CS137 } \\
1 & \text { K40 }
\end{array}
$$

3.000
23.000

140.000
9.660

300.670

1480.000
0.630
15.250

77.800
1.242
0.981
0.909

$\begin{array}{ll}\text { W } & \text { W } \\ \text { A } & \text { A } \\ \text { A } & \text { W }\end{array}$

Matrix: WA Water $\mathrm{Bq} ; \mathrm{L}$

$\begin{array}{ll}1 & \text { AM241 } \\ 1 & \text { CO60 } \\ 1 & \text { CS } 134 \\ 1 & \text { CS137 } \\ 1 & \text { Gross Alpha } \\ 1 & \text { Gross Beta } \\ 1 & \text { PU238 } \\ 1 & \text { PU239 } \\ 1 & \text { SR90 } \\ 1 & \text { U234 } \\ 1 & \text { U238 }\end{array}$

$$
\begin{array}{r}
3.090 \\
263.000 \\
57.000 \\
77.000 \\
221.000 \\
909.000 \\
3.710 \\
1.840 \\
7.900 \\
3.050 \\
3.120
\end{array}
$$

$$
\begin{array}{r}
3.043 \\
268.670 \\
60.200 \\
81.430 \\
210.000 \\
900.000 \\
4.331 \\
2.070 \\
8.690 \\
3.323 \\
3.370
\end{array}
$$

$$
\begin{array}{r}
0.082 \\
9.710 \\
1.860 \\
4.280 \\
21.000 \\
90.000 \\
0.117 \\
0.074 \\
0.420 \\
0.114 \\
0.140
\end{array}
$$

$$
\begin{aligned}
& \text { A } \\
& \text { A } \\
& \text { A } \\
& \text { A } \\
& \text { A } \\
& \text { A } \\
& \text { A } \\
& \text { A } \\
& \text { A }
\end{aligned}
$$




\section{QAP 56 Results by Laboratory}

Lab: EG INEEL TRA Radioanalytical Laboratory, Scoville

\begin{tabular}{|c|c|c|c|c|c|}
\hline $\begin{array}{l}\text { No. } \\
\text { Test }\end{array}$ & Radionuclide & $\begin{array}{c}\text { Reported } \\
\text { Value }\end{array}$ & $\begin{array}{c}\text { Reported } \\
\text { Error }\end{array}$ & $\begin{array}{l}\text { EML } \\
\text { Value }\end{array}$ & $\begin{array}{l}\text { EVL } \\
\text { Error }\end{array}$ \\
\hline
\end{tabular}

Matrix: Al Air Filter $\mathrm{Bq} /$ filter

$\begin{array}{ll}\text { 1 } & \text { AM241 } \\ 1 & \text { CO60 } \\ 1 & \text { CS137 } \\ 1 & \text { MN54 } \\ \text { I } & \text { PU238 } \\ \text { I } & \text { PU239 } \\ \text { I } & \text { SR90 } \\ \text { I } & \text { U234 } \\ \text { I } & \text { U238 }\end{array}$

Matrix: SO Soil Bq/ $\mathrm{kg}$

$\begin{array}{ll}1 & \text { AC228 } \\ \text { I } & \text { AM241 } \\ 1 & \text { BI212 } \\ 1 & \text { BI214 } \\ 1 & C S 137 \\ 1 & \text { K40 } \\ 1 & \text { PB212 } \\ \text { I } & \text { PB214 } \\ 1 & \text { PU239 } \\ 1 & \text { SR90 } \\ 1 & \text { TH234 } \\ 1 & \text { U234 } \\ 1 & \text { U238 }\end{array}$

Matrix: VE Vegetation $\mathrm{Bq} / \mathrm{kg}$

$\begin{array}{ll}1.042 & \mathrm{~A} \\ 1.091 & \mathrm{~A} \\ 1.098 & \mathrm{~A} \\ 1.134 & \mathrm{~A} \\ 1.097 & \mathrm{~A} \\ 1.110 & \mathrm{~A} \\ 0.844 & \mathrm{~A} \\ 0.972 & \mathrm{~A} \\ 0.986 & \mathrm{~A}\end{array}$

A

2.000

3.000

0.005

0.013

0.130

0.020

$\begin{array}{rr}0.088 & 0.005 \\ 30.520 & 0.652 \\ 28.230 & 0.701 \\ 38.530 & 0.867 \\ 0.057 & 0.001 \\ 0.187 & 0.003 \\ 4.832 & 0.184 \\ 0.297 & 0.004 \\ 0.298 & 0.004\end{array}$

$\begin{array}{rr}10.000 & 51.167 \\ 0.900 & 10.927 \\ 24.000 & 53.430 \\ 7.000 & 53.933 \\ 100.000 & 1326.670 \\ 80.000 & 62\} .670 \\ 6.000 & 51.100 \\ 8.000 & 54.367 \\ 1.900 & 19.098 \\ 2.800 & 53.756 \\ 26.000 & 89.300 \\ 5.300 & 93.885 \\ 5.600 & 96.778\end{array}$

1.941
0.373
5.215
2.249
66.510
33.860
2.753
2.249
0.706
1.446
6.837
7.767
8.410

1.134

1.043

1.142
0.723

1.033

0.788

1.213

1.196

1.173

0.964

1.389

0.897
0.898

$A$
$A$
$A$
$N$
$A$
$N$
$W$
$A$
$W$
$A$
$A$
$A$
$A$

W

A

A

$A$

W

A

$\begin{array}{ll}1 & \text { AM241 } \\ 1 & \text { CM214 } \\ 1 & \text { CO60 } \\ 1 & \text { CS137 } \\ 1 & \text { K40 } \\ 1 & \text { PU238 } \\ 1 & \text { PU239 } \\ 1 & \text { SR90 }\end{array}$

2.400
1.300
9.100
317.000
740.000
0.250
3.540
520.000

0.180
0.110
1.900
25.000
100.000
0.040
0.250
17.000

2.228
1.320
11.230
313.667
864.330
0.257
3.543
586.280

0.216
0.164
0.677
15.910
47.220
0.046
0.377
11.140

1.077
0.985
0.810
1.011
0.856
0.974
0.999
0.887

Matrix: WA Water $\mathrm{Bq} / \mathrm{L}$

$\begin{array}{ll}1 & \text { AM24l } \\ 1 & \text { CO60 } \\ 1 & \text { CS134 } \\ 1 & \text { CSI37 } \\ 1 & \text { GROSS ALPHA } \\ 1 & \text { GROSS BETA } \\ 1 & \text { PU238 }\end{array}$

1.570
360.000
3.000
57.000
360.000
1080.000
0.480

0.100
30.000
0.400
4.000
29.000
58.000
0.037

0.021
12.400
0.200
2.929
37.500
103.000
0.032

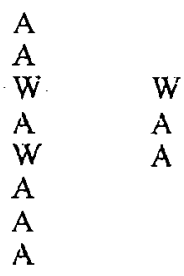

Values for elemental uranium are reported in $\mu \mathrm{g} /$ filter, $\mathrm{g}$, or $\mathrm{mL}$.

Evaluation: $A=$ Acceptable, $W=$ Acceptable with Warning, $N=$ Not Acceptable

If the evaluation system is not appropriate for the types of analyses performed in your lab, apply a site specific evaluation. 
QAP 0203

EML-617

June 2002

\section{QAP 56 Results by Laboratory}

Lab: EG INEEL TRA Radioanalytical Laboratory, Scoville

\begin{tabular}{|c|c|c|c|c|c|c|c|c|}
\hline $\begin{array}{l}\text { No. } \\
\text { Test }\end{array}$ & Radionuclide & $\begin{array}{l}\text { Reported } \\
\text { Value }\end{array}$ & $\begin{array}{l}\text { Reported } \\
\text { Error }\end{array}$ & $\begin{array}{l}\text { EML } \\
\text { Value }\end{array}$ & $\begin{array}{l}\text { EML } \\
\text { Error }\end{array}$ & $\frac{\text { Reported }}{\text { EML }}$ & Evaluation & $\begin{array}{c}\text { QAP 55 } \\
\text { Evaluation }\end{array}$ \\
\hline
\end{tabular}

Matrix: WA Water $B q / L$

\begin{tabular}{|c|c|c|c|c|c|c|}
\hline PU239 & 4.490 & 0.270 & 4.219 & 0.172 & 1.064 & A \\
\hline SR 90 & 6.520 & 0.280 & 7.579 & 0.176 & 0.860 & A \\
\hline U234 & 1.270 & 0.070 & 1.402 & 0.056 & 0.906 & A \\
\hline U238 & 1.300 & 0.070 & 1.381 & 0.079 & 0.941 & A \\
\hline
\end{tabular}

Values for elemental uranium are reported in $\mu \mathrm{g}$ filter, $\mathrm{g}$, or $\mathrm{mL}$. 


\section{U.S. DEPARTMENT OF COMMERCE \\ National Institute of Standards and Technology \\ Gaithersburg, MD}

\section{REPORT OF TRACEABILITY}

\section{Bechtel BWXT Idaho, LLC Idaho Falls, Idaho}

Test Identification

NRIP02-SS

Matrix Description

${ }^{241} \mathrm{Am},{ }^{238} \mathrm{Pu},{ }^{230} \mathrm{Th},{ }^{90} \mathrm{Sr},{ }^{238} \mathrm{U}$ in Soil ${ }^{1}$

Test Activity Range

$30 \mathrm{mBq}-$ sample $^{-1}$ to $300 \mathrm{mBq}$-sample ${ }^{-1}$

Reference Time

12:00 EST, April 1; 2002

Measurement Results

\begin{tabular}{|c|c|c|c|c|c|}
\hline Nuclide & \multicolumn{2}{|c|}{ NIST Value ${ }^{2,3}$} & \multicolumn{2}{|c|}{ Reported Value $^{4}$} & Difference $^{5}$ \\
\hline & $\begin{array}{c}\text { Massic Activity } \\
\mathrm{Bq} \cdot \mathrm{g}^{-1}\end{array}$ & $\begin{array}{l}\text { Relative Expanded } \\
\text { Uncertainty }(\%, \mathrm{k}=2)\end{array}$ & $\begin{array}{l}\text { Massic Activity } \\
\mathrm{Bq} \cdot \mathrm{g}^{-1}\end{array}$ & $\begin{array}{c}\text { Relative Expanded } \\
\text { Uncertainty }(\%, k=2)\end{array}$ & $(\%)$ \\
\hline $\begin{array}{l}{ }^{241} \mathrm{Am} \\
{ }^{238} \mathrm{Pu} \\
{ }^{230} \mathrm{Th} \\
{ }^{238} \mathrm{U} \\
{ }^{90} \mathrm{Sr}\end{array}$ & $\begin{array}{l}2.127 \\
1.893 \\
3.187 \\
8.012 \\
8.428\end{array}$ & $\begin{array}{l}0.67 \\
1.14 \\
0.61 \\
0.63 \\
0.74 \\
\end{array}$ & $\begin{array}{l}2.12 \\
1.65 \\
\text { NR } \\
7.82 \\
7.46\end{array}$ & $\begin{array}{l}10.8 \\
16.4 \\
\mathrm{NA} \\
11.4 \\
13.1 \\
\end{array}$ & $\begin{array}{l}-0.4 \\
-13 \\
\text { NA } \\
-2.4 \\
-11 \\
\end{array}$ \\
\hline \multicolumn{6}{|c|}{$\mathbf{N A}=$ Not Applicable } \\
\hline \multicolumn{6}{|c|}{ Measurement Method } \\
\hline \multirow{2}{*}{\multicolumn{2}{|c|}{ Activity Measurements }} & \multicolumn{2}{|c|}{ NIST $^{6}$} & \multicolumn{2}{|c|}{ Reporting Laboratory? } \\
\hline & & \multicolumn{2}{|c|}{$\begin{array}{c}\text { Alpha- and Beta-Spectrometry } \\
\text { Mass Spectrometry }\end{array}$} & \multicolumn{2}{|c|}{ Alpha- and Beta-Spectrometry } \\
\hline
\end{tabular}

Evaluation (per ANSI N42.22).

\begin{tabular}{|c|c|c|}
\hline Nuclide & \multicolumn{2}{|c|}{ N42.22 } \\
\hline & $\begin{array}{c}\text { ANSI N42.22 } \\
\text { Traceable }\end{array}$ & $\begin{array}{c}\text { Traceability } \\
\text { Limit } \\
\text { ( } \pm \text { Percent) }\end{array}$ \\
\hline${ }^{241} \mathbf{A m}$ & Yes & 16 \\
${ }^{238} \mathbf{P u}$ & Yes & 22 \\
${ }^{230} \mathbf{T h}$ & NA & NA \\
${ }^{238} \mathrm{U}$ & Yes & 17 \\
${ }^{90} \mathrm{Sr}$ & Yes & 17 \\
\hline
\end{tabular}

Samples Distributed

Reporting Data Received
May 17, 2002

August 19, 2002
For the Director

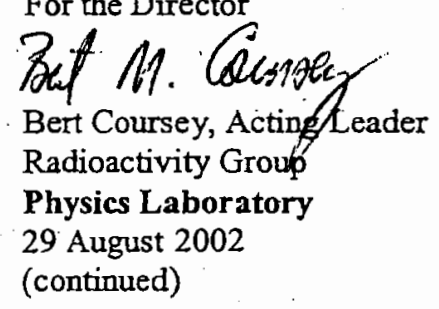




\section{U.S. DEPARTMENT OF COMMERCE National Institute of Standards and Technology \\ Gaithersburg, MD}

\section{REPORT OF TRACEABILITY}

\section{Lockheed Martin Idaho Technologies Company Idaho Falls, Idaho}

Test Identification

Matrix Description

Test Activity Range

Reference Time
NRIP02-GF

${ }^{241} \mathrm{Am},{ }^{238} \mathrm{Pu},{ }^{90} \mathrm{Sr},{ }^{238} \mathrm{U},{ }^{230} \mathrm{Th}$ on Glass-Fiber Filters ${ }^{1}$

$30 \mathrm{mBq} \cdot \mathrm{sample}^{-1}$ to $300 \mathrm{mBq} \cdot \mathrm{sample}^{-1}$

12:00 EST, April 1, 2002

Measurement Results

\begin{tabular}{|c|c|c|c|c|c|}
\hline Nuclide & \multicolumn{2}{|c|}{ NIST Value ${ }^{2,3}$} & \multicolumn{2}{|c|}{ Reported Value ${ }^{4}$} & Difference $^{5}$ \\
\hline & $\begin{array}{c}\text { Massic Activity } \\
\mathrm{Bq} \bullet \mathrm{g}^{-1}\end{array}$ & $\begin{array}{l}\text { Relative Expanded } \\
\text { Uncertainty }(\%, 2 s) \\
\end{array}$ & $\begin{array}{l}\text { Massic Activity } \\
\text { Bq. } \mathrm{g}^{-1}\end{array}$ & $\begin{array}{l}\text { Relative Expanded } \\
\text { Uncertainty }(\%, 2 \mathrm{~s}) \\
\end{array}$ & $( \pm \%$ Bias $)$ \\
\hline${ }^{241} \mathrm{Am}$ & 2.437 & 0.67 & 2.40 & 10.6 & $\overline{-1.5}$ \\
\hline${ }^{238} \mathrm{Pu}$ & 2.168 & 1.14 & 2.00 & 10.6 & -7.8 \\
\hline${ }^{238} \mathrm{U}$ & 9.527 & 0.63 & 9.26 & 10.8 & -2.8 \\
\hline${ }^{90} \mathrm{Sr}$ & 9.653 & 0.77 & 10.01 & 10.8 & +3.7 \\
\hline${ }^{230} \mathrm{Th}$ & 3.650 & 0.61 & 3.66 & 12.2 & +0.3 \\
\hline \multicolumn{3}{|c|}{ NA $=$ Not Applicable } & & \multicolumn{2}{|c|}{ NR $=$ Not Reported } \\
\hline \multicolumn{6}{|c|}{ Methods } \\
\hline \multirow{2}{*}{\multicolumn{2}{|c|}{ Activity Measurements }} & \multicolumn{2}{|c|}{ NIST $^{6}$} & \multicolumn{2}{|c|}{ Reporting Laboratory ${ }^{7}$} \\
\hline & & \multicolumn{2}{|c|}{$\begin{array}{l}\text { Alpha- and Beta-Spectrometry } \\
\text { Mass Spectrometry }\end{array}$} & \multicolumn{2}{|c|}{ Alpha- and Beta-Spectrometry } \\
\hline
\end{tabular}

Evaluation (per ANSI N42.22)

\begin{tabular}{|c|c|c|}
\hline Nuclide & \multicolumn{2}{|c|}{$\mathbf{N 4 2 . 2 2}^{8}$} \\
\hline & $\begin{array}{c}\text { ANSI N42.22 } \\
\text { Traceable }\end{array}$ & $\begin{array}{c}\text { Traceability } \\
\text { Limit } \\
( \pm \text { Percent })\end{array}$ \\
\hline${ }^{241} \mathrm{Am}$ & Yes & 16 \\
${ }^{238} \mathrm{Pu}$ & Yes & 15 \\
${ }^{238} \mathrm{U}$ & Yes & 16 \\
${ }^{90} \mathrm{Sr}$ & Yes & 17 \\
${ }^{230} \mathrm{Th}$ & Yes & 18 \\
\hline
\end{tabular}

Samples Distributed

8 March 2002

Reporting Data Received
16 May 2002
For the Director

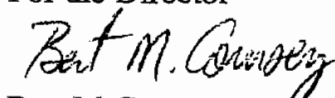

Bert M.Coursey, Affing Leader Radioactivity Group

Physics Laboratory

19 June 2002

(continued) 
